\title{
Colloidal Nanocrystal Quantum Dots for High-Efficiency Optoelectronic Conversions
}

\author{
Yiqiang Zhang \\ West Virginia University
}

Follow this and additional works at: https://researchrepository.wvu.edu/etd

\section{Recommended Citation}

Zhang, Yiqiang, "Colloidal Nanocrystal Quantum Dots for High-Efficiency Optoelectronic Conversions" (2012). Graduate Theses, Dissertations, and Problem Reports. 3571.

https://researchrepository.wvu.edu/etd/3571

This Dissertation is protected by copyright and/or related rights. It has been brought to you by the The Research Repository @ WVU with permission from the rights-holder(s). You are free to use this Dissertation in any way that is permitted by the copyright and related rights legislation that applies to your use. For other uses you must obtain permission from the rights-holder(s) directly, unless additional rights are indicated by a Creative Commons license in the record and/ or on the work itself. This Dissertation has been accepted for inclusion in WVU Graduate Theses, Dissertations, and Problem Reports collection by an authorized administrator of The Research Repository @ WVU.

For more information, please contact researchrepository@mail.wvu.edu. 


\title{
Colloidal Nanocrystal Quantum Dots for High-Efficiency Optoelectronic Conversions \\ by
}

Yiqiang Zhang

Dissertation Proposal submitted to the Benjamin M. Statler College of Engineering and Mineral Resources at West Virginia University in partial fulfillment of the requirements for the degree of

Doctor of Philosophy

In

Electrical Engineering

\author{
Committee Members \\ Xian-An Cao, Ph.D., Committee Chairperson \\ Jeremy Dawson, Ph.D. \\ Parviz Famouri, Ph.D. \\ Lawrence Hornak, Ph.D. \\ Xiaodong Shi, Ph.D.
}

Lane Department of Computer Science and Electrical Engineering

Morgantown, West Virginia 2012

Keywords: Quantum Dot; Light-Emitting Diode; Solar Energy Conversion; Ligand Exchange

Copyright 2012 Yiqiang Zhang 


\section{ABSTRACT}

\section{Colloidal Nanocrystal Quantum Dots for High-Efficiency Optoelectronic Conversions by Yiqiang Zhang}

Monodispersed colloidal quantum dots (QDs) synthesized by low-cost solution methods have many attractive properties, including high wavelength tunability, efficient luminescence, narrow emission bandwidth, and strong broadband absorption. They therefore offer a new class of materials for efficient optoelectronic conversions in various devices, including light-emitting diodes (LEDs), photodetectors, and thin-film solar cells. Despite the recent rapid progress, the development of QD-based devices is still in its infancy. For instance, both the external quantum efficiency of QD-LEDs and power conversion efficiency of QD solar cells reported in the literature are only a few percent, far below those of state-ofthe-art inorganic and organic semiconductor devices. The performance of QD-based devices is largely limited by the insulating and bulky organic ligands of QDs, which are necessary for colloidal synthesis, but create large interparticle spacing and interfacial energy barriers, impeding charge transport and injection as well as exciton dissociation and energy transfer.

This work aims to enhance the energy conversion efficiencies in QD-based optoelectronic devices through surface modification of QDs and device structure optimization, which can enable efficient electronic coupling and energy transfer between QDs and surrounding materials. First, an in-house capability of colloidal QD synthesis by the hot-injection method was developed. High-quality CdSe, CdS and CdTe core QDs with sharp excitonic absorption features and narrow PL bandwidths were synthesized. Growing a ZnS shell surrounding the CdSe core QDs via the SILAR method increased the photoluminescence quantum yield (PL QY) from 10\% to 50\%. Furthermore, the ZnS shell was replaced by a graded $\mathrm{CdS} / \mathrm{Zn}_{0.5} \mathrm{Cd}_{0.5} \mathrm{~S} / \mathrm{ZnS}$ multishell, leading to reduced interfacial defects and an improved PL QY $\sim 65 \%$. Second, CdSe and CdSe/ZnS QDs with inorganic metal chalcogenide ligands $\left(\mathrm{SnS}_{4}{ }^{4-}\right.$ ) were synthesized by an organic-to-inorganic ligand exchange process. The $\mathrm{SnS}_{4}$ ligands significantly enhanced the inter-QD electronic coupling in QD solids, but caused a substantial reduction in the PL efficiency. Finally, the applicability of CdSe QDs with organic and inorganic ligands for optoelectronic applications is evaluated through detailed optical, electrical and optoelectronic characterization. LEDs based on a QDs/organic materials hybrid structure were fabricated and characterized. The best result was obtained from the LEDs based on organically-capped CdSe/ZnS QDs with a layer of blue phosphorescent FIrpic dyes as efficient exciton harvesters and energy donors. Precise control of the concentration of the donors and their distance from the QD layer led to complete exciton energy transfer and efficiency enhancement by a factor of 2.5. Meanwhile, the $\mathrm{SnS}_{4}$-capped QDs were found to retain strong excitonic absorption. Under a $150 \mathrm{~W}$ Xe lamp illumination, the photocurrent response of an ITO/QDs/Al structure increased by several orders of magnitude after the ligand exchange. These findings bode well for the applicability of colloidal QDs with metal chalcogenide ligands to efficient energy conversion in low-cost thin-film solar cells. 


\section{ACKNOWLEDGEMENTS}

I would like to thank my parents for their constant love and support of all of my endeavors. Without them, I would not have made it this far.

I would especially like to thank my advisor Prof. Xian-An Cao for offering me the opportunity to work on very interesting research topic of the quantum dot (QD) materials and QD-based optoelectronic devices, for providing excellent experimental facilities, for his kind

support and guidance on my thesis work, and for his expertise, motivation, enthusiasm, and immense knowledge on research.

I wish to thank Prof. Lawrence A. Hornak, Prof. Xiaodong Shi, Prof. Parviz Famouri, and Prof. Jeremy Dawson for serving on my committee and their invaluable suggestions to improve my research work.

I also wish to thank Dr. Wuming Yan and Dr. Ming Li for providing me kind help and valuable advice of setting up the Schlenk line system for the synthesis of QDs. In addition, I wish to thank Mr. Tom Trovato of Trovato Mfg., Inc. for training me how to use and maintain the thermal evaporator, and for helping us design and integrate the MBraun glovebox with the thermal evaporator. Furthermore, I wish to thank Dr. Weiqiang Ding and Dr. Kolin Brown of WVU Shared Research Facilities for their patient training and timely technical advice.

All my lab colleagues at the Microfabrication and Nanosystems Laboratory made it a wonderful place to work. In particular, I would like to thank Dr. Gaoyu Zhong, Dr. Yi Yang, Henry Andagan, Zhenyu Jiang, Hong Guo, Rajeev Acharya, and Yifei Lu, for their friendship and help in the past 4 years.

Finally, and most importantly, I thank my wife and friend, Zhenhua $\mathrm{Wu}$, and my two sons, for giving me up to long hours and late nights in lab. They have been a constant source of strength. To them I dedicated this dissertation. 


\section{TABLE OF CONTENTS}

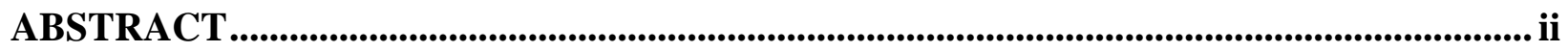

ACKNOWLEDGEMENTS ...........................................................................................................ii

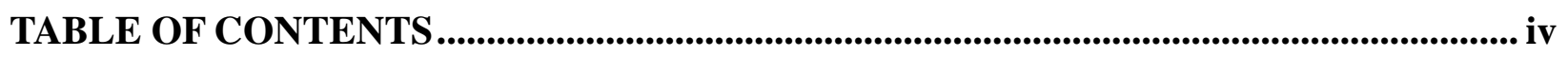

LIST OF FIGURES .......................................................................................................... vii

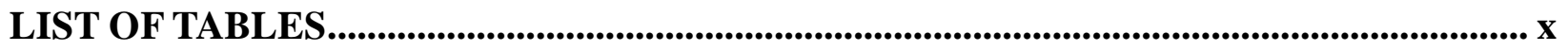

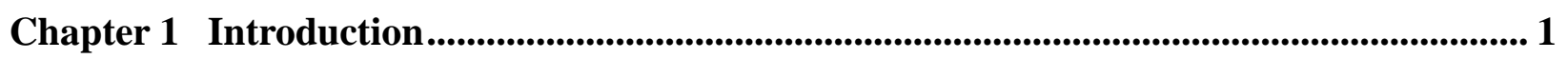

1.1 Colloidal Nanocrystal QDs ....................................................................................... 1

1.1.1 Quantum Confinement Effects in QDs ................................................................. 2

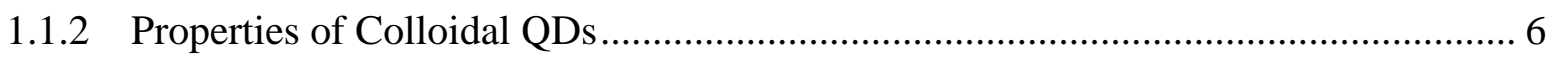

1.1.3 Colloidal Core and Core/Shell QDs Synthesis ........................................................... 8

1.2 Colloidal QDs for Energy Conversions ........................................................................ 14

1.2.1 Design of Surface Ligands of QDs for Energy Conversions ..................................... 15

1.2.2 QD-based Light-Emitting Diodes ............................................................................. 24

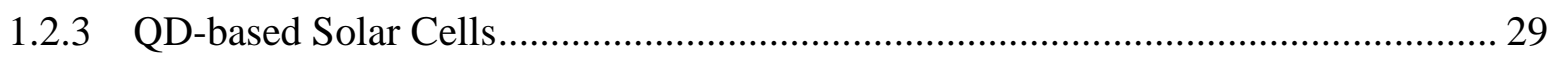

1.3 Challenges Facing QD-based Devices.......................................................................... 34

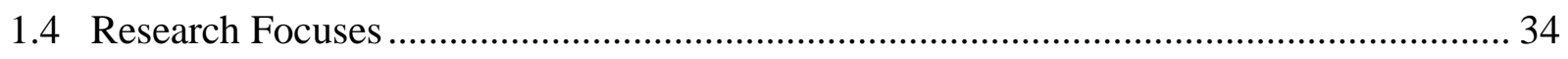

Chapter 2 Synthesis and Characterization of Colloidal Semiconductor Quantum Dots ... 36

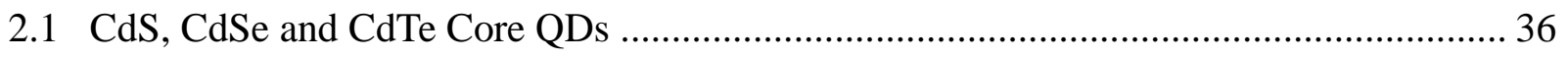

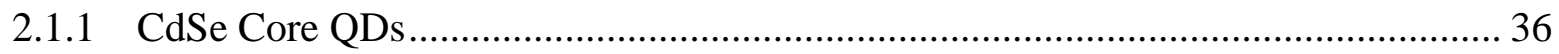

2.1.2 CdS Core QDs................................................................................................... 40

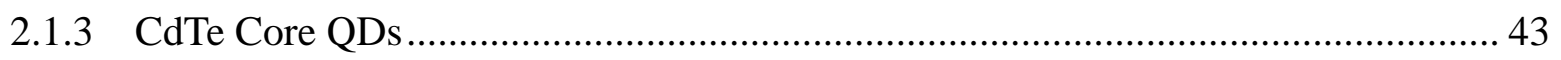

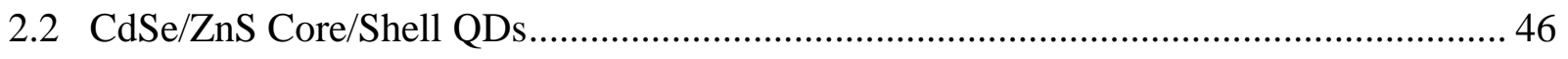




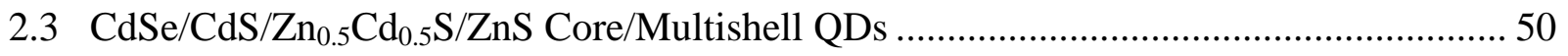

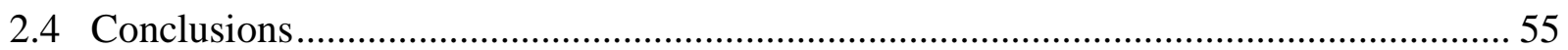

Chapter 3 Development of High-Efficiency CdSe/ZnS QD-LEDs .......................................... 56

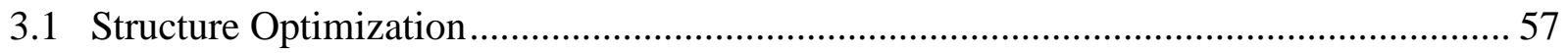

3.2 Performance Enhancement with Phosphorescent Donors .................................................. 61

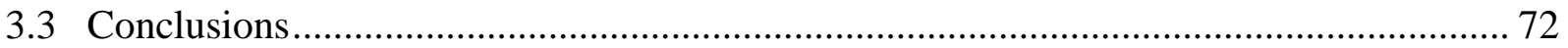

Chapter 4 Synthesis and Characterization of CdSe QDs with Inorganic Ligands.............. 73

4.1 Ligand Exchange in Hydrazine Solvent …………....................................................... 75

4.2 Ligand Exchange in Ammonia Hydroxide Solvent ....................................................... 78

4.3 Optical Characterization of MCC-capped Quantum Dots Thin Film ................................. 82

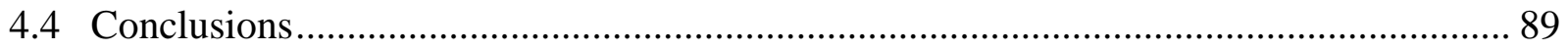

Chapter 5 Quantum Dots with Inorganic Ligands for Energy Conversions......................... 91

5.1 CdSe Based Core/Multishell QDs with MCC Ligands for Light Emission ....................... 92

5.2 Quantum Dots with MCC Ligands for Photovoltaic Conversion........................................ 96

5.2.1 Electrical Characterization of ITO/MCC-capped QDs/Al........................................... 98

5.2.2 Photocurrent Response of ITO/MCC-capped QDs/Al ............................................. 101

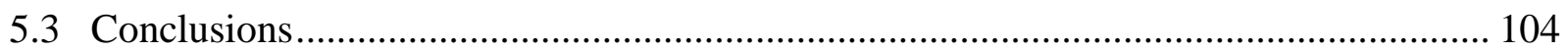

Chapter 6 Conclusions and Future Work …………............................................................. 105

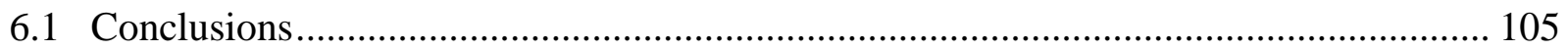

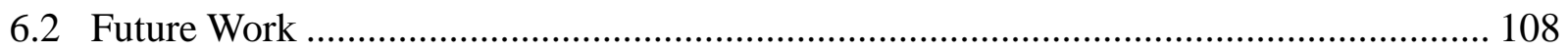

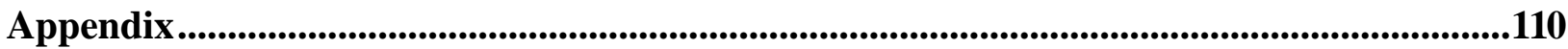

A.1 Synthesis of CdSe/ZnS Core/Shell Quantum Dots with 5 Monolayers of ZnS Shell ......110

A.1.1 Synthesis of CdSe Core QDs ..................................................................................110

A.1.2 Preparation of the Precursor Solution for the SILAR Method.....................................110

A.1.3 Calculations of the Amount of Precursor Solutions for Shell Growth........................111 
A.1.4 Synthesis of CdSe/ZnS Core/Shell QDs Using the SILAR Method.........................112

A.2 Characterization of Quantum Yields of Colloidal Quantum Dots .................................113

References...........................................................................................................................................115

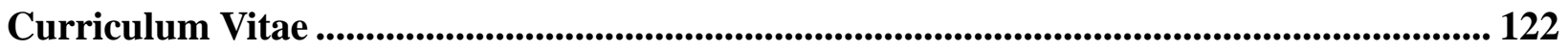




\section{LIST OF FIGURES}

Figure 1.1 Colloidal CdSe core QDs .................................................................................... 2

Figure 1.2 Illustration of quantum confinement effects........................................................ 4

Figure 1.3 Quantum confinement effects in QDs ............................................................... 5

Figure 1.4 Size-dependent optical properties of colloidal CdSe QDs ....................................... 7

Figure 1.5 Cartoon of the typical set-up to synthesize monodisperse colloidal semiconductor

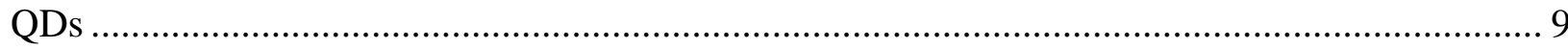

Figure 1.6 Schematic showing the stages of nucleation and growth for synthesis of monodisperse

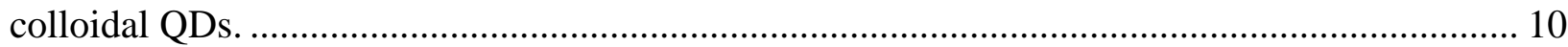

Figure 1.7 Structure and energy diagram of CdSe/ZnS core/shell QDs .................................... 12

Figure $1.8 \mathrm{CdSe}$ CdS core/shell QDs synthesized by SILAR technique.................................... 14

Figure 1.9 Thermo-gravitational (TGA) analysis of ODA-capped CdSe/CdZnS/ZnS

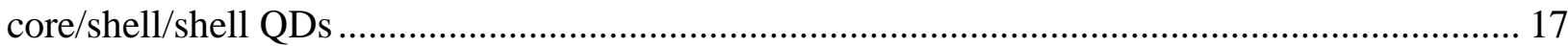

Figure 1.10 Energy levels tuning of InAs QDs through ligand exchange ................................ 21

Figure 1.11 Stable colloidal solutions of MCC-capped QDs ................................................... 23

Figure 1.12 Cross-section structure of a typical QD-LED ............................................... 25

Figure 1.13 Development of QD-LEDs (green circles) and OLEDs (orange circles) over the

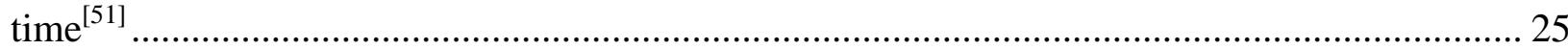

Figure 1.14 Electroluminescence spectra and structures for two QD-LEDs ............................. 26

Figure 1.15 QD-LEDs with EL tunable over the entire visible spectrum from $460 \mathrm{~nm}$ (blue) to

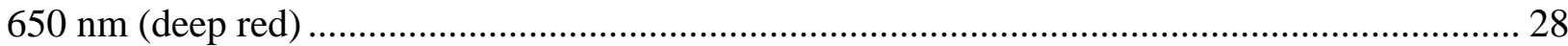

Figure 1.16 Energy diagram of QD-LEDs and full-color QD display ................................... 29

Figure 1.17 All inorganic CdTe/CdSe QD solar cell......................................................... 30

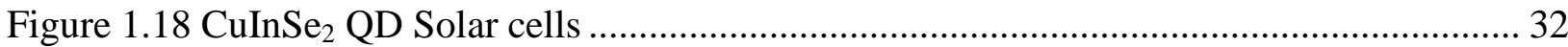

Figure 1.19 Structure, performance and schematics of Schottky solar cell based on colloidal

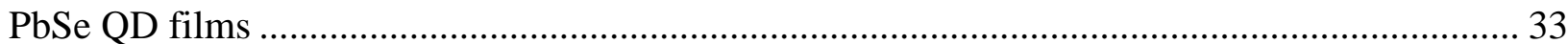

Figure 2.1 Cartoon depicting the hot-injection method for synthesis of CdSe core QDs. .......... 37 Figure 2.2 Temporal evolution of (a) absorption and (b) PL spectra of CdSe QDs samples taken

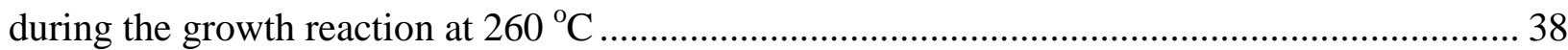
Figure 2.3 Temporal dependence of the particle size during the growth of CdSe QDs at $260{ }^{\circ} \mathrm{C} 39$ Figure 2.4 Absorption and PL spectrum of CdSe QDs emitting green light (530 nm) ............. 40 Figure 2.5 Temporal evolution of absorption spectra of CdS QDs samples taken during the

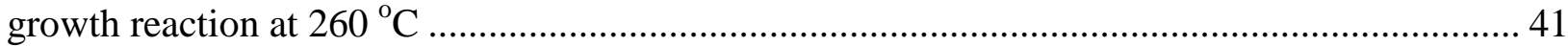
Figure 2.6 Temporal dependence of the particle size during the growth of CdS QDs at $260{ }^{\circ} \mathrm{C} .42$ Figure 2.7 Absorption and PL spectrum of CdS QDs emitting deep blue light (435 nm)........... 43 Figure 2.8 Temporal evolution of (a) absorption and (b) PL spectra of CdTe QDs samples taken during the growth reaction at $250{ }^{\circ} \mathrm{C}$. There is no evident PL spectra tested for the QDs samples grown for $1800 \mathrm{sec}$ or $3600 \mathrm{sec}$. 
Figure 2.9 Temporal dependence of the particle size during the growth of CdTe QDs at $250{ }^{\circ} \mathrm{C} 45$ Figure 2.10 Absorption and PL spectrum of CdTe QDs emitting deep red light $(670 \mathrm{~nm})$......... 46 Figure 2.11 Illustration of CdSe/ZnS core/shell QDs synthesis using the SILAR method ........... 47 Figure 2.12 Absorption spectra of CdSe core and CdSe/ZnS core/shell QDs with different

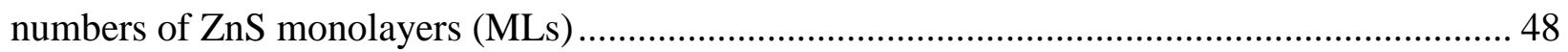
Figure 2.13 Evolution of the photoluminescence quantum yield of CdSe/ZnS core/shell QDs... 49 Figure 2.14 PL spectra and absorption spectra of green-emitting CdSe core QDs and CdSe/ZnS QDs 50

Figure 2.15 Schematic representation of band structure of CdSe/CdS/Zn ${ }_{0.5} \mathrm{Cd}_{0.5} \mathrm{~S} / \mathrm{ZnS}$ core/multishell QDs, demonstrating the gradual increase in the bandgap from the CdSe core to

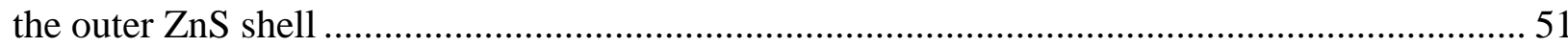

Figure 2.16 Evolution of the absorption spectra upon consecutive growth of the shells .............. 53 Figure 2.17 Evolution of the photoluminescence quantum yield of $\mathrm{CdSe} / \mathrm{CdS} / \mathrm{Zn}_{0.5} \mathrm{Cd}_{0.5} \mathrm{~S} / \mathrm{ZnS}$ core/multishell QDs with 2MLs of CdS shell, 3MLs of $\mathrm{Zn}_{0.5} \mathrm{Cd}_{0.5} \mathrm{~S}$ shell, and 2MLs of $\mathrm{ZnS}$ shell

Figure 2.18 PL spectra and absorption spectra of red-emitting CdSe core QDs and $\mathrm{CdSe} / \mathrm{CdS} / \mathrm{Zn}_{0.5} \mathrm{Cd}_{0.5} \mathrm{~S} / \mathrm{ZnS}$ core/multishell QDs (as-prepared) ................................................... 55 Figure 3.1 Trovato evaporation system integrated with MBraun glovebox for fabrication of QD-

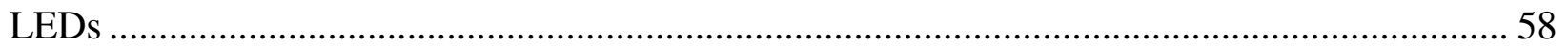
Figure 3.2 a) The cross-section and b) the energy diagram of the QD-LEDs ............................... 59

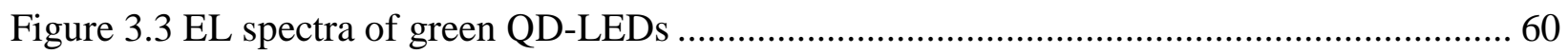
Figure 3.4 I-V Characteristics of CdSe/ZnS QD-LEDs with different concentration of QD

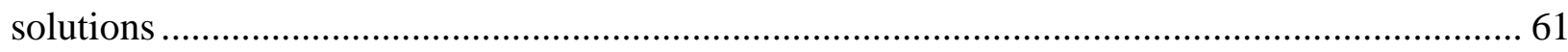
Figure 3.5 Absorption spectrum of green CdSe/ZnS QDs in hexane shown along with PL

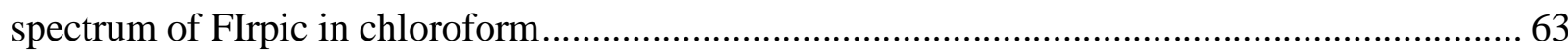
Figure 3.6 (a) Layer structure of a CdSe/ZnS QD-LED with a FIrpic-doped CBP energy-donor

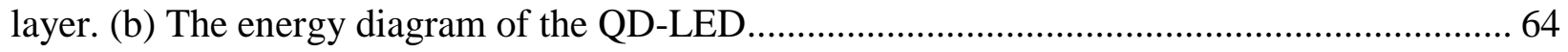
Figure 3.7 Normalized EL spectra of green CdSe/ZnS QD-LEDs ................................................. 65 Figure 3.8 (a) I-V characteristics and (b) normalized EL spectra, recorded at $10 \mathrm{~mA} / \mathrm{cm} 2$ of

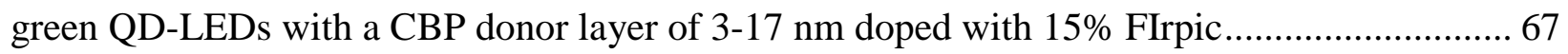

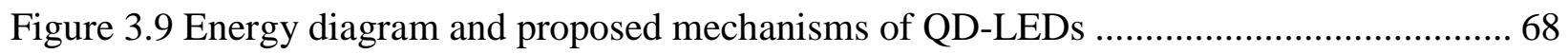

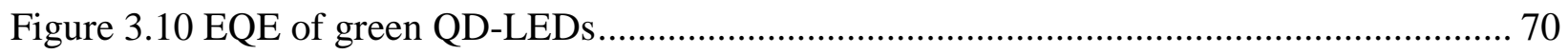

Figure 4.1 Schematics of the ligand exchange process............................................................... 74

Figure 4.2 Schematics of organic-to-MCC ligands exchange in hydrazine solvent...................... 75 Figure 4.3 FTIR spectra of CdSe QDs capped with original organic ligands and after ligand exchange with $\left(\mathrm{N}_{2} \mathrm{H}_{5}\right)_{4} \mathrm{Sn}_{2} \mathrm{~S}_{6}$. The spectra are normalized to the amount of absorbing materials

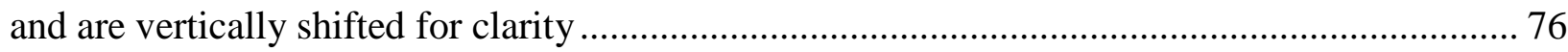
Figure 4.4 Normalized (a) absorption and (b) PL spectra of CdSe core QDs with organic ODA ligands in hexane solvent or with inorganic $\mathrm{Sn}_{2} \mathrm{~S}_{6}{ }^{4-}$ ligands in hydrazine solvent ....................... 77 Figure 4.5 Normalized (a) absorption and (b) PL spectra of CdSe/ZnS core/shell QDs with organic ODA ligands in hexane solvent or with inorganic $\mathrm{Sn}_{2} \mathrm{~S}_{6}{ }^{4-}$ ligands in hydrazine solvent 78 
Figure 4.6 Schematics of organic-to-MCC ligand exchange in ammonia hydroxide solvent ...... 79 Figure 4.7 Normalized (a) absorption and (b) PL spectra of CdSe core QDs with organic ODA ligands in hexane solvent or with inorganic $\mathrm{SnS}_{4}$ ligands in ammonia hydroxide solvent .......... 80 Figure 4.8 Normalized (a) absorption and (b) PL spectra of CdSe/CdS/ZnS core/multishell QDs with organic ODA ligands in hexane solvent or with inorganic $\mathrm{SnS}_{4}$ ligands in ammonia hydroxide solvent. 81

Figure 4.9 FTIR spectra of CdSe QDs capped with original organic ligands and after ligand exchange with $\mathrm{Na}_{4} \mathrm{SnS}_{4}$. The spectra are normalized to the amount of absorbing materials and are vertically shifted for clarity.

Figure 4.10 Normalized (a) absorption and (b) PL spectra of the films of CdSe core QDs with organic ODA or inorganic $\mathrm{SnS}_{4}$ ligands. The PL of QDs with $\mathrm{SnS}_{4}$ ligands is completely quenched at room temperature. 83

Figure 4.11 Normalized (a) absorption and (b) PL spectra of the films of CdSe/CdS/ZnS core/multishell QDs with organic ODA or inorganic $\mathrm{SnS}_{4}$ ligands. 85

Figure 4.12 Absorption spectra of the films of ODA-capped (a) CdSe core QDs and (b) $\mathrm{CdSe} / \mathrm{CdS} / \mathrm{ZnS}$ core/multishell QDs as well as $\mathrm{SnS}_{4}$-capped (c) CdSe core QDs and (d) $\mathrm{CdSe} / \mathrm{CdS} / \mathrm{ZnS}$ core/multishell QDs annealed at different temperatures. The spectra are shifted in the vertical direction for clarity. 86

Figure 4.13 PL intensity of the films of CdSe core QDs and CdSe/CdS/ZnS core/multishell QDs with different ligands as a function of annealing temperature. 88

Figure 5.1 Scheme of the LBL assembly method to fabricate QD-LEDs. ............................... 94 Figure 5.2 (a) Absorption spectra of 5, 10, 20, and 30 bilayers of $\mathrm{SnS}_{4}$-capped QDs/PDDA films. The discrete excitonic absorption peaks can be seen from the inset of enlarged absorption spectra. (b) Absorbance at the shoulder position of $450 \mathrm{~nm}$ vs. the number of QDs/PDDA billayers...... 95 Figure 5.3 The best device performance of QD-LEDs comprising of 10 bilayers of $\mathrm{SnS}_{4}$-capped QDs/PDDA films. (a) I-V Characteristics and (b) normalized EL spectrum taken at $100 \mathrm{~mA} / \mathrm{cm}^{2}$ and PL spectrum of 10 bilayers of $\mathrm{SnS}_{4}$-capped QDs/PDDA films. ...................................... 96 Figure 5.4 Cross-section of an ITO/CdSe QDs/Al structure. ............................................... 97 Figure 5.5 I-V characteristics of ITO/CdSe QDs/Al structures. The QDs, with (a) ODA and (b) $\mathrm{SnS}_{4}$ ligands, were annealed at different temperatures prior to $\mathrm{Al}$ deposition 99 Figure 5.6 Evolution of current in ITO/CdSe QDs/Al structures under a constant bias of $5 \mathrm{~V}$. The QDs were annealed at $250{ }^{\circ} \mathrm{C}$ prior to $\mathrm{Al}$ deposition. 100 Figure 5.7 I-V characteristics of as-fabricated ITO/CdSe QDs/Al structures with (a) ODA and (b) $\mathrm{SnS}_{4}$-capped QDs, as a result of different scans. 101

Figure 5.8 Photocurrent spectral responses and absorption spectra of ITO/CdSe QDs/Al structures comprising QDs with (a) ODA and (b) $\mathrm{SnS}_{4}$ ligands. The QDs were annealed at 150 ${ }^{\circ} \mathrm{C}$ prior to $\mathrm{Al}$ deposition. 102 Figure 5.9 Photocurrent spectral responses of ITO/CdSe QDs/Al structures with (a) ODA and (b) $\mathrm{SnS}_{4}$-capped QDs, which were annealed at different temperatures prior to Al deposition ........ 103 


\section{LIST OF TABLES}

Table 1.1 Different type of surface ligands for colloidal QDs ............................................... 18 


\section{Chapter 1 Introduction}

The recent energy crisis and global warming concerns have led to great interest in developing efficient device technologies for renewable energy generation and energy conservation. Next-generation of solid-state lighting and displays demands an exceptional solution with pure color, low power consumption, and low-cost manufacturing. On the other hand, the commercialization of solar energy usage requires high-efficiency and low-cost solar cells. The availability of monodispersed colloidal nanocrystal quantum dots (QDs) synthesized by low-cost solution methods has offered a new class of materials for developing such energy conversion technologies. This dissertation evaluates the applicability of CdSe-based colloidal QDs for low-cost and high efficiency optoelectronic applications, including light-emitting diodes (LEDs) and solar cells, which may make a significant technological impact in the near future.

\subsection{Colloidal Nanocrystal QDs}

Colloidal nanocrystal QDs are nanometer-sized crystalline semiconductor material consisting of hundreds to a few thousand atoms each. Their size ranges from 2 to $20 \mathrm{~nm}$ in diameter. Colloidal QDs are typically synthesized in a stable colloidal solution using a chemical process. Figure 1.1 (a) is a schematic view of the structure of an exemplary CdSe QD, whose surface is capped with long-chain organic ligands. In a high resolution transmission electron micrograph (TEM) image shown in Figure 1.1 (b), a well-defined atomic lattice can be seen. Due to the organic capping layer, the growth of each QD can be well confined under the same circumstance. So, all of the QDs possess similar sizes and compositions for a single synthesis. Figure 1.1 (c) is TEM of $8.5 \mathrm{~nm}$ diameter red CdSe QDs array ${ }^{[1]}$. As seen, the QDs are selfassembled into close-packed and ordered arrays with good monodispersity, demonstrating a narrow size distribution. Today, the colloidal semiconductor QD material systems under 
intensive research include II-VI (CdSe, CdTe, CdS $)^{[2]}$, IV-VI $\left(\mathrm{PbS}^{[3]}, \mathrm{PbSe}^{[4]}, \mathrm{PbTe}^{[5]}\right)$, and III-V (InP, InAs) ${ }^{[6]}$ QDs.
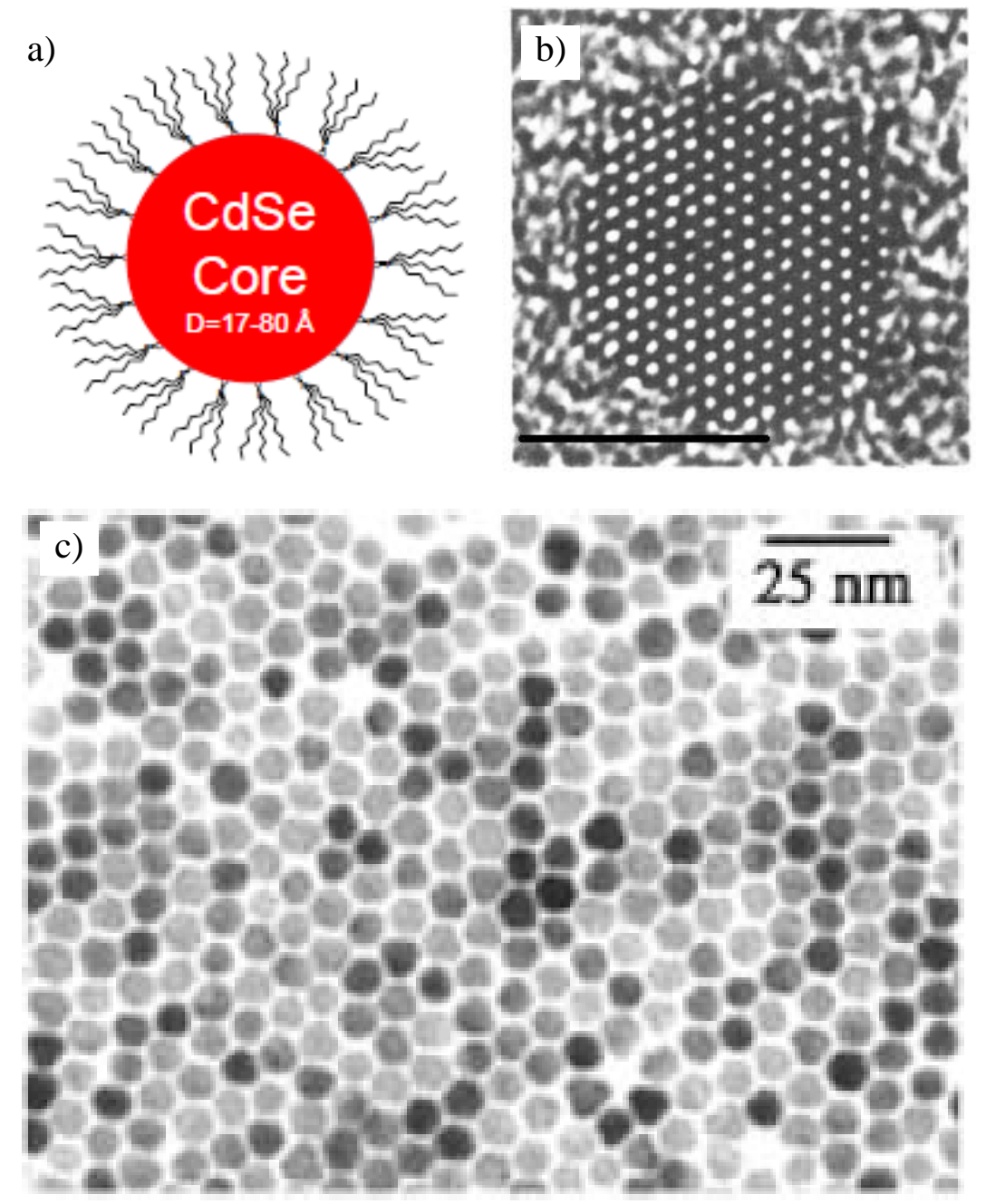

\section{Figure 1.1 Colloidal CdSe core QDs}

a) Schematic showing the structure of CdSe core QDs. The outer ligands layer is composed by TOPO. b) High resolution transmission electron micrograph of a CdSe QDs, where actual atom can be seen scale bar=50 $\AA .{ }^{[7]} \mathrm{c}$ ) Transmission electron micrograph of $8.5 \mathrm{~nm}$ diameter CdSe QDs ${ }^{[1]}$.

\subsubsection{Quantum Confinement Effects in QDs}

A semiconductor material is defined by the band gap separating the conduction and valence bands. The color of light emitted by the semiconductor material is determined by the width of the band gap. In bulk materials, the band gap width is a fixed parameter determined by 
the material itself. The situation changes, however, in the case of nanoscale semiconductor materials with sizes comparable to their exciton Bohr radius, which is the distance separating an electron-hole pair. This means the size of semiconductor nanocrystals is comparable with the spatial extent of the electronic wave function. Electrons are confined within a potential energy box and the electron kinetic energy increases as the box gets smaller. This is the result of the uncertainty relation $\Delta x \Delta p \geq \hbar / 2$, one of the fundamental principles of quantum mechanics. Setting an energy box length $L=\Delta x$ and electron momentum $p=m v$, the minimum kinetic energy of electrons is $E=\hbar^{2} / 2 m * L^{2}$ (Plank's constant $\hbar$ and electron effective mass $m^{*}$ ). This formula expresses the basic relationship between the electron energy and the size L of semiconductor crystal, known as the quantum confinement effect.

The quantum confinement effect in semiconductors was first seen in the 1970 ' ${ }^{[8]}$, when molecular beam epitaxy was developed as a technique for growing thin layers of semiconductor materials at atomic precision thickness, known as quantum wells (QWs). QWs confine electrons in the dimension perpendicular to the layer. When the QW thickness becomes comparable to the exciton Bohr radius, the quantum confinement effects will take place, leading to energy subbands (Figure 1.2). One purpose of fabricating QW structures is to tune the energy of bandto-band transitions by adjusting the well thickness, in order to tune the optical properties of semiconductors. Also, the one-dimension confinement leads to better optical performance by increasing the probability of recombination of electrons and holes. These two advantages of QWs lead to better performance of QW-based optoelectronic devices such as laser diodes, compared to devices without quantum confinement. Today, most semiconductor laser products used in optical communications and optical data storage systems are based on QW heterostructures. The theoretical and experimental studies of quantum confinement effects in QWs have been extended to even lower-dimension nanostructures including quantum wires and quantum dots. Figure 1.2 describes the development from bulk semiconductors to quantum 
wells, quantum wires, and finally to three-dimensionally confined quantum dots, along with the corresponding changes in band structure and density of states.
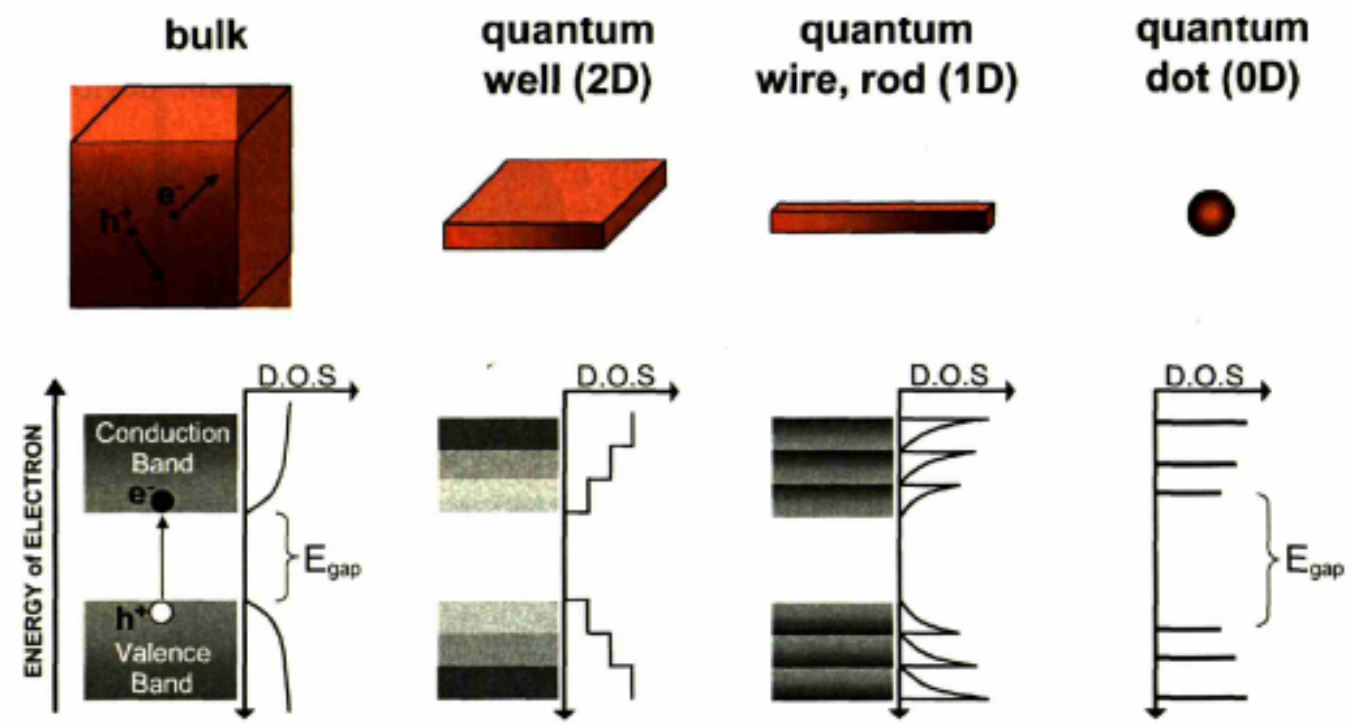

Figure 1.2 Illustration of quantum confinement effects

In bulk semiconductor, motion of carriers in bulk semiconductor is unrestricted and the band structure is associated with a density states proportional to $\mathrm{E}^{1 / 2}$. In quantum wells, motion of the carriers is confined in two dimensions resulting in a constant density of states. In quantum wire and rods, motion of the carriers is restricted to one dimension and the density of states begins to sharpen. In quantum dots, the carriers are confined in all three dimensions leading to discrete energy levels. This picture was taken from J. S. Steckel's Dissertation at Massachusetts Institute of Technology, 2006.

If the size of colloidal semiconductor QDs is smaller than the size of the Bohr exciton radius, the charge carriers are spatially confined to and delocalized over the entire space of the QDs. The confinement of carriers in all three dimensions lead to a collapse of the continuous energy bands of a bulk semiconductor into discrete energy states (Figure 1.3). These energy states are directly related to the QD size: the quantum confinement results in the series of electron and hole states with S, P, and D symmetries identical to the discrete energy levels of hydrogen atoms. In an unexcited QD, the energy gap between the lowest unoccupied $\left(1 \mathrm{~S}_{\mathrm{e}}\right)$ and the highest occupied $\left(1 \mathrm{~S}_{\mathrm{h}}\right)$ energy states is much larger than $\mathrm{k}_{\mathrm{B}} \mathrm{T}(\sim 26 \mathrm{meV}$ at $300 \mathrm{~K})$, so electrons cannot be thermally excited into $1 \mathrm{~S}_{\mathrm{e}}$ state. The external excitation of the semiconductor 
QD, for example, photoexcitation or electrical excitation, must be used to create excess carriers to make QDs emit light. For a spherical QD with radius R, the model shown in Figure 1.3 (c) predicts that the size-dependent contribution to the energy gap is simply proportional to $1 / R^{2}$, indicating that the energy gap increases as the QD size decreases. This model directly explains that, within the quantum confinement regime, the optical and electronic properties of QDs are strongly dependent on their size.

(a) CdSe Bulk Semiconductor

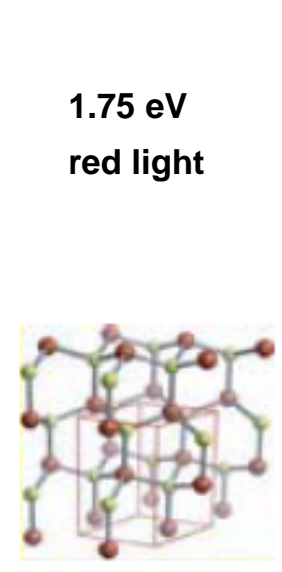

(c)

$$
\begin{gathered}
E_{\mathrm{g}}(\mathrm{QD})=E_{\mathrm{g} 0}+\frac{\hbar^{2} \pi^{2}}{2 m_{\mathrm{eh}} R^{2}} \\
m_{\mathrm{eh}}=\frac{m_{\mathrm{e}} m_{\mathrm{h}}}{m_{\mathrm{e}}+m_{\mathrm{h}}} \\
m_{\mathrm{e}}=\text { effective electron mass } \\
m_{\mathrm{h}}=\text { effective hole mass }
\end{gathered}
$$

(b) CdSe Quantum Dot (QD)

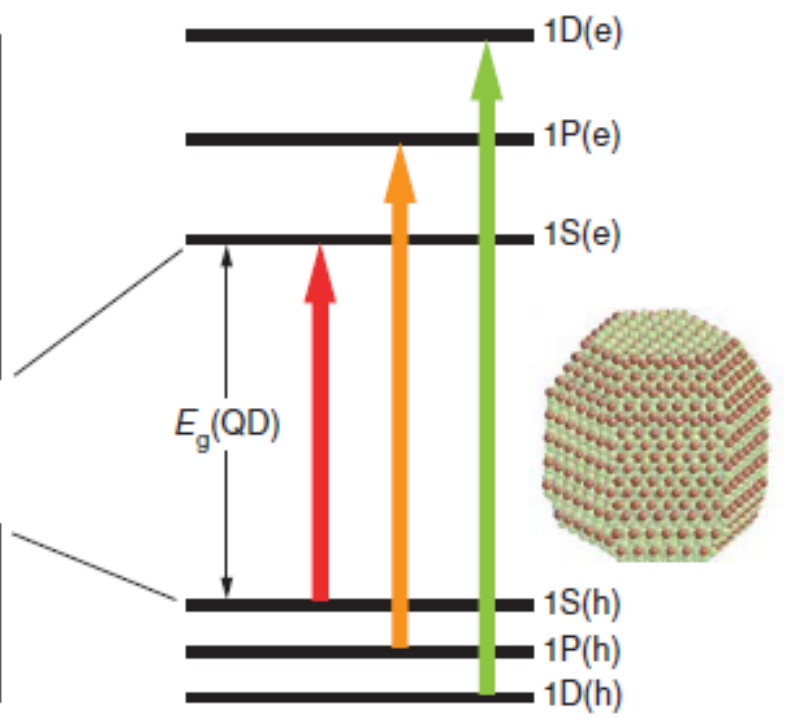

(d)

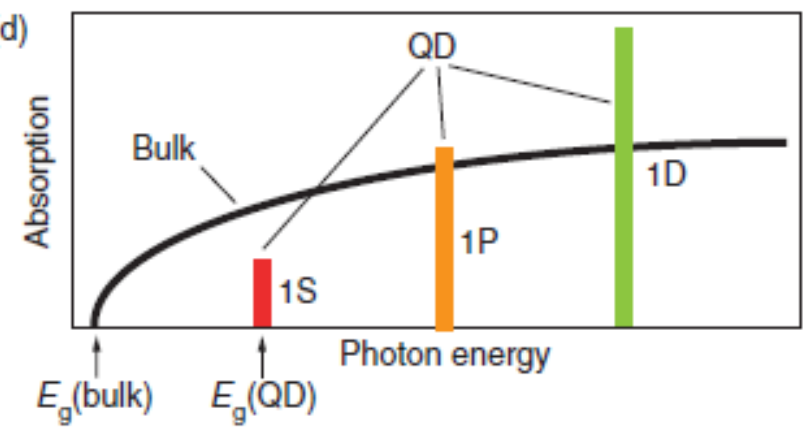

\section{Figure 1.3 Quantum confinement effects in QDs}

a) A bulk semiconductor such as CdSe has continuous conduction and valence energy bands separated by a "fixed" energy gap, Eg(bulk). Electrons normally occupy all states up to the edge of the valence band, whereas states in the conduction band are empty. b) A QD is characterized by discrete atomic-like states with energies that are determined by the QD radius R. These well-separated QD states can be labeled with atomic-like notations, such as 1S, 1P, and 1D. c) The expression for the size-dependent separation between the lowest electron and hole QD 
states—Eg(QD), the QD energy gap—was obtained with the spherical "quantum box" model. d) This schematic represents the continuous absorption spectrum of a bulk semiconductor (black line) compared with a discrete absorption spectrum of a QD (colored bars). ${ }^{[9]}$

\subsubsection{Properties of Colloidal QDs}

The most striking property of semiconductor QDs is the size-dependent optical properties due to the quantum confinement effect (Figure 1.4). As the size of colloidal semiconductor QDs is reduced, the electronic excitations shift to higher energy, and the absorption and photoluminescence (PL) spectra of QDs display blue shifts. Colloidal CdSe QDs with a bulk band gap of $1.75 \mathrm{eV}$ can be tuned to emit light spanning the entire visible spectrum. As shown in Figure 1.4 (a), CdSe QDs of $2 \mathrm{~nm}$ in diameter emit blue light, whereas CdSe QDs of $8 \mathrm{~nm}$ emit red light. In addition, the full widths at half maximum (FWHM) of PL spectra of all QDs are around $30 \mathrm{~nm}$ (Figure $1.4 \mathrm{c}$ ). This low FWHM value suggests that QDs in an ensemble are highly uniform in size and shape, leading to color-saturated PL. The size-dependent, colorsaturated emission of QDs across the visible spectrum is unsurpassed by any class of organic chromophores, making them very attractive for both biological imaging ${ }^{[10]}$ and LEDs $^{[11]}$. Meanwhile, the light absorption properties of colloidal semiconductor QDs are also quite unique. Just like their PL, the absorption property can be tuned by adjusting the diameter of QDs as well. As seen in Figure 1.4 (c), the absorption spectrum of CdSe QDs with a diameter of $20 \mathrm{~nm}$ is similar to that of bulk CdSe material, due to little quantum confinement in such large QDs. Remarkable blue shift and sharp electronic transitions are obvious in the absorption spectra of CdSe QDs with a diameter less than $10 \mathrm{~nm}$ (the exciton Bohr diameter for CdSe is 9.6 nm). Up to five electronic transitions are resolved in the absorption spectra, indicating very narrow size distribution of the CdSe QDs. Other unique absorption features of QDs include strong absorption cross sections, giant extinction coefficients, and broad continuous absorptions. Colloidal QDs are thus being explored for developing high-efficiency solar cells ${ }^{[12]}$ and photodetectors ${ }^{[13]}$. 

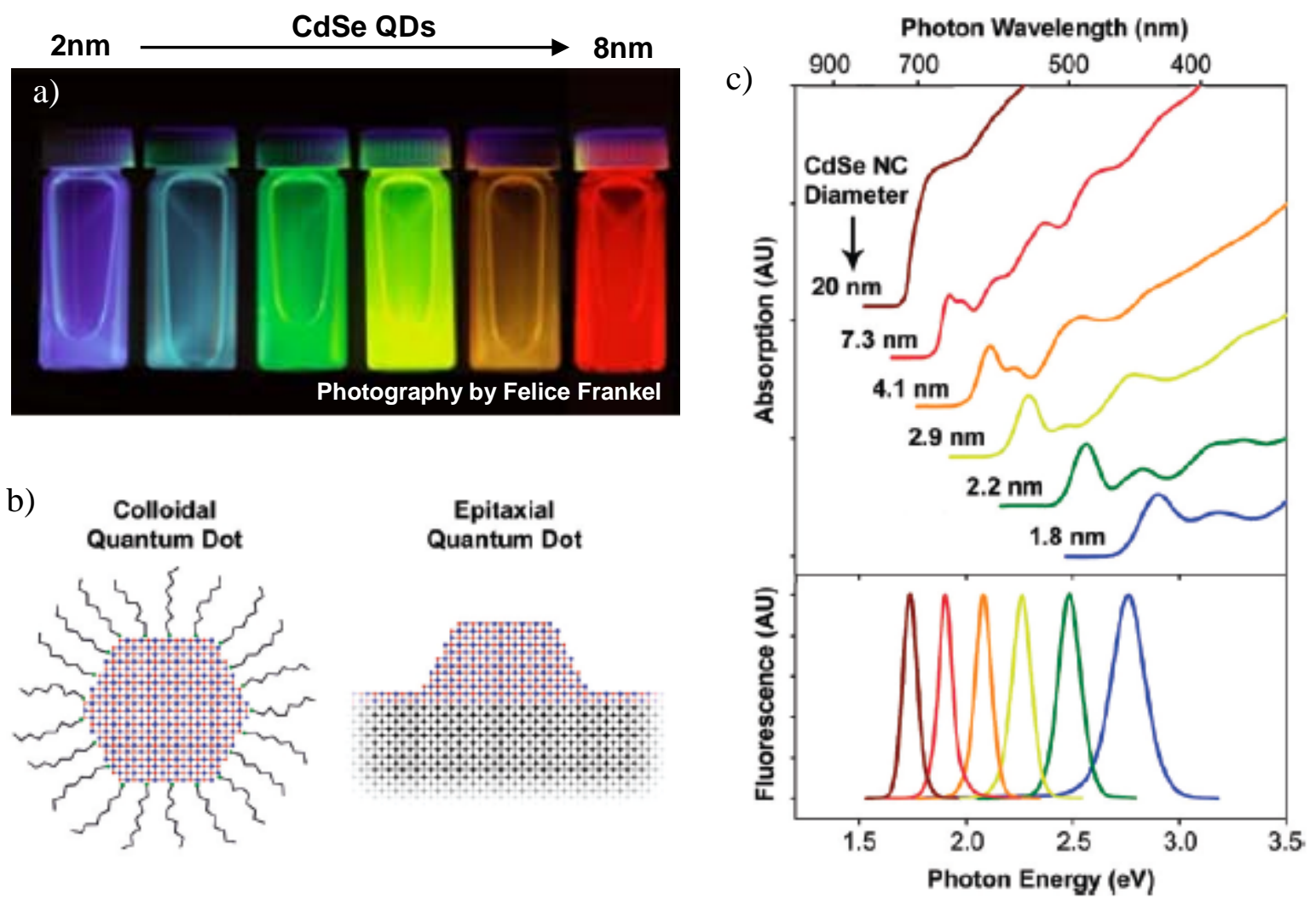

Figure 1.4 Size-dependent optical properties of colloidal CdSe QDs

a) Different size of fluorescent CdSe QDs (2 $\mathrm{nm}$ to $8 \mathrm{~nm}$ in diameter) dispersed in hexane. b) Comparison of a colloidal QD synthesized in high-boiling organic solution and an epitaxial QD grown on a substrate by high vacuum deposition technique ${ }^{[14]}$. c) Absorption (upper) and photoluminescence (lower) spectra of CdSe semiconductor QDs demonstrating quantum confinement effect and size tunability ${ }^{[14]}$.

Nowadays, QDs are commonly synthesized as colloids suspended in solution or epitaxially deposited on solid substrates (Figure 1.4 b). Epitaxial QDs can be fabricated with a wide range of shapes and sizes by high-vacuum deposition techniques, such as molecular beam epitaxy or metallorganic vapor phase epitaxy, and directly buried into optoelectronic devices. Fabrication of expitaxial QDs requires complicated and expensive technological processes. In contrast, colloidal QDs with uniform size and shape can be synthesized using low-cost colloidal chemical processes, offering opportunities for inexpensive device fabrication on versatile substrates by solution processing such as spin coating, dip coating, or inkjet printing. Colloidal QDs are thus suitable for low-cost and large-area manufacturing. ${ }^{[15]}$ In addition, a larger band 
gap semiconductor shell can be grown on the colloidal QD core surface, providing very good surface passivation and protection from photo-oxidations. The core/shell QD structure (for example, CdSe/ZnS QDs) is desirable for most optoelectronic device applications, due to its enhanced quantum efficiency as well as increased stability against harsh processing conditions. $^{[16]}$

\subsubsection{Colloidal Core and Core/Shell QDs Synthesis}

The pioneering work of colloidal semiconductor QDs synthesis was conducted by Dr. Brus at Bell Laboratories. ${ }^{[17]}$ Motivated by developing high-quality QDs for solar energy conversion and photocatalysis after the first oil crisis, they designed an aqueous and ionic chemistry for QDs synthesis. In addition to the tuning of the absorption spectra and redox potential of the QDs based on quantum confinement, it was expected that the high surface to volume ratio of QDs would improve carrier collection efficiency or surface reaction yields. However, the aqueous colloidal synthetic approach only occurred under low temperature (lower than $150{ }^{\circ} \mathrm{C}$ even under reflux cooling) and produced limited surface passivation, leading to poor size distribution and low quantum efficiency of QDs. ${ }^{[18]}$ Hence, the development of a better synthetic method is required to synthesize QDs of good quality. 


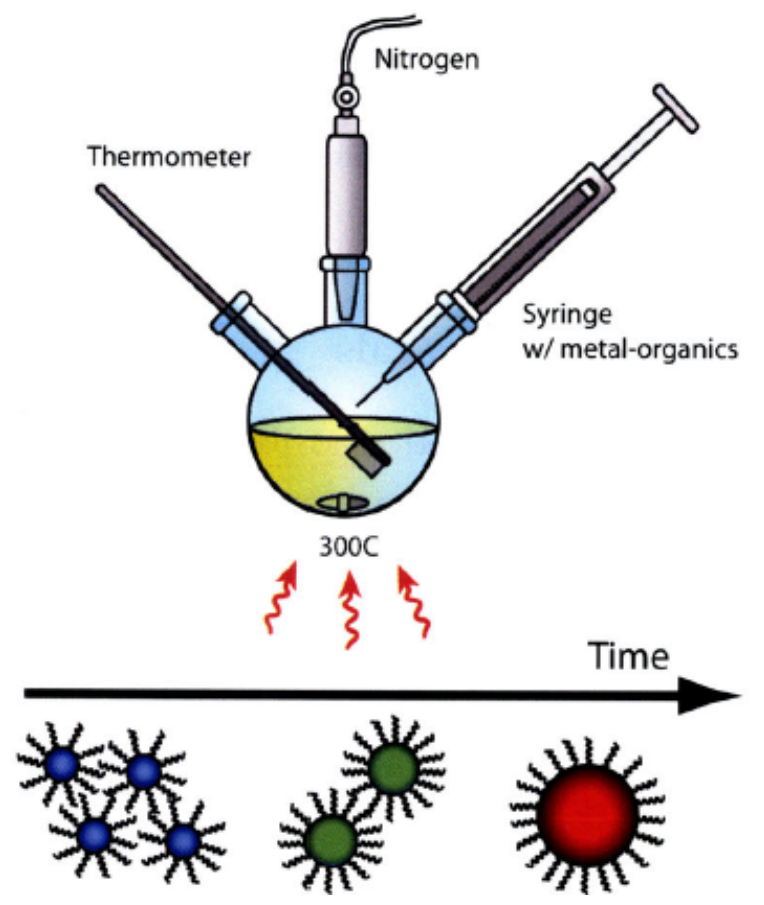

Figure 1.5 Cartoon of the typical set-up to synthesize monodisperse colloidal semiconductor QDs

This typical apparatus consists of a three-neck flask with a magnetic stir bar, a condenser connected to a Schlenk line, a thermometer, and a rubber septum through which a syringe of precursors can be rapidly injected into the hot solvent. As the growth time prolongs, the tiny particles will grow from blue, green, to red color emission. This picture was taken from P. O. Anikeeva’s Dissertation at Massachusetts Institute of Technology, 2009.

In 1993, Murray and co-workers made a breakthrough and synthesized monodisperse CdE (E=sulfur, selenium, tellurium) QDs by high-temperature $\left(300-360{ }^{\circ} \mathrm{C}\right)$ pyrolysis of organometallic precursors (dimethylcadmium) in a coordinating solvent of trioctylphosphine oxide (TOPO) (Figure 1.5). ${ }^{[2]}$ The success of this work relied on the effective utility of a classic model proposed by LaMer and Dinegar for monodisperse colloidal growth. ${ }^{[19]}$ Based on their theory, a temporally discrete nucleation event followed by a slow and controlled growth on the existing nuclei is required for the synthesis of monodisperse colloidal QDs. This is usually achieved by the so-called hot-injection technique: a rapid injection of concentrated precursors into a hot solvent. As illustrated in Figure 1.6, once the concentration of the injected precursor exceeds the nucleation threshold, a short nucleation would burst to partially relieve the supersaturation. The nuclei formed in the nucleation process would subsequently grow over 
reaction time. Another distinct growth phase known as Ostwald ripening will occur, if the growth of QDs lasts long enough. This process involves the dissolution of small QDs due to their high surface energy and the further growth of larger ones. As a result, the average QD size increases over time, and the QD concentration decreases. Taking advantage of Ostwald ripening, so-called “focusing” of the size distribution during QD growth, was described by Dr. Peng and coworkers, and has proven to be beneficial for the preparation of high-quality QDs. ${ }^{[1]}$ Using this organometallic route, colloidal QDs based on II-VI (CdSe, CdTe, CdS), III-V (InP, InAs), and IV-VI (PbS, PbSe, PbTe) semiconductor materials have been successfully synthesized. ${ }^{[20]}$

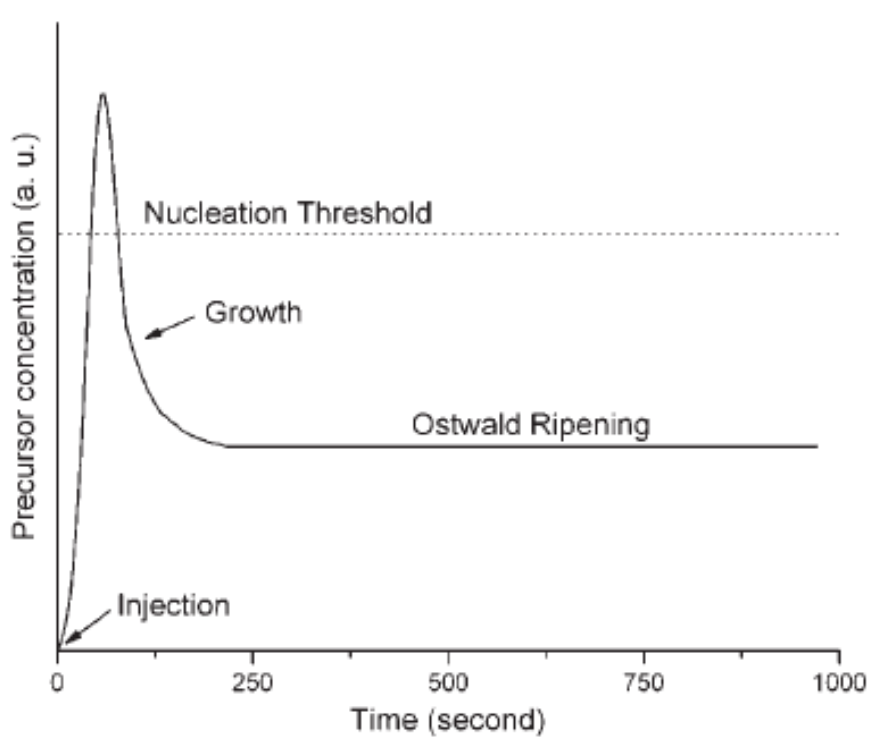

Figure 1.6 Schematic showing the stages of nucleation and growth for synthesis of monodisperse colloidal QDs.

This illustration is based on the LaMer and Dinegar model. In the plot, the relationship between concentration of precursors and time of reaction depicts how a sudden increase in concentration of precursors caused the nucleation of particles, which then grow over reaction time. ${ }^{[19]}$

Although this organometallic synthetic approach was widespreadly employed in the synthesis of monodisperse QDs with different sizes and shapes, the starting materials, such as dimethylcadmium, are extremely toxic, pyrophoric, explosive, and expensive. Therefore, researchers explored greener approaches to synthesize high-quality semiconductor QDs. In 2000, 
Dr. Peng and co-workers introduced "green” and stable metal salts to replace dimethylcadmium and inexpensive and nontoxic 1-octadencene (ODE) to replace the TOPO solvent. ${ }^{[21]}$ This green approach was achieved without losing any of the unique properties of QDs prepared by previous organometallic synthetic method. ${ }^{[22]}$ The metal salts have been found to be converted into reactive precursors for QD growth. For instance, cadmium oxide (CdO) is converted into cadmium oleate when degassing and then heating up to $250{ }^{\circ} \mathrm{C}$ under inert atmosphere in the presence of oleic acid (OA), and the cadmium oleate then reacts with trioctylphosphine selenide (TOP-Se) to form CdSe QDs. This strategy can also be applied to other material systems, such as II-VI (CdTe, CdS), III-V (InP, InAs), and IV-VI (PbS, PbSe, PbTe) semiconductor QDs, as outlined by reactions $1-3$. The synthesis is typically carried out at a temperature between $200{ }^{\circ} \mathrm{C}$ and $300{ }^{\circ} \mathrm{C}$, and in the presence of long-chain alkylphosphines (e.g., TOP), alkylphosphine oxides (e.g., TOPO), and alkylamines (e.g., hexadecylamine (HDA)), or alkylphosphonic acids as ligands to passivate the surface of QDs.

$$
\begin{aligned}
& \mathrm{Cd}\left(\mathrm{CH}_{3} \mathrm{COO}\right)_{2}+\left(\mathrm{C}_{8} \mathrm{H}_{17}\right)_{3} \mathrm{PS} \stackrel{300^{\circ} \mathrm{C} \text {, HDA-TOPO-TOP }}{\longrightarrow} \mathrm{CdS} \text { QDs } \\
& \mathrm{InCl}_{3}+\left[\left(\mathrm{CH}_{3}\right)_{3} \mathrm{Si}\right]_{3} \mathrm{P} \stackrel{260^{\circ} \mathrm{C}, \mathrm{TOP}}{\longrightarrow} \mathrm{InP} \text { QDs } \\
& \mathrm{Pb}\left(\mathrm{CH}_{3} \mathrm{COO}\right)_{2}+\left(\mathrm{C}_{8} \mathrm{H}_{17}\right)_{3} \mathrm{PSe} \stackrel{180^{\circ} \mathrm{C} \text {, oleic acid }}{\longrightarrow} \text { PbSe QDs }
\end{aligned}
$$

To some extent, both the nucleation and growth stage can be controlled and the QDs are separated by the ligands. The ligands produce a steric barrier for adding additional precursors to the surface of a growing crystal, significantly slowing the growth kinetics. The desirable choices of the concentration and type of ligands should be made to prevent uncontrolled nucleation and growth, as well as complete growth suppression. The above synthetic approach provides a strong control over the whole nucleation and growth procedures, and thus the synthesis recipe can be optimized to grow QDs with a narrow size distribution and an ideal emission wavelength. The key process parameters include the concentration and type of ligands, reaction temperature, 
duration of the crystal growth, and the anionic/cationic ratio of precursors. By adjusting one or more of these parameters, the size of colloidal QDs can be tuned across a large range while a retaining good size distribution.

As discussed previously, the surface of colloidal QDs is passivated by a monolayer of organic ligands to provide good interaction with the solvent as well as eliminate many of surface traps. However, typical organically passivated QDs may still exist a large number of surface electronic states which act as non-radiative relaxation pathways for charge carriers, therefore reducing luminescence efficiencies. To overcome the problems, the QDs may be capped with a shell of a different semiconductor material with a larger bandgap, resulting in core/shell QDs. In this way, the shell provides a physical barrier between the active core and surrounding medium, and thus makes QDs less sensitive to environmental changes, surface chemistry, and photooxidation. ${ }^{[23]}$ The shell also further provides an efficient passivation of surface trap states of the core QDs, leading to a significantly enhancement of $\mathrm{PL}^{[24]}$ and electroluminescence $(\mathrm{EL})^{[25]}$. For example, when a ZnS shell is grown onto CdSe core QDs, both electron and hole wave functions are strongly confined inside the core (Figure 1.7), giving rise to a 2 to 4 fold increase in PL quantum yields (QYs). ${ }^{[26]}$

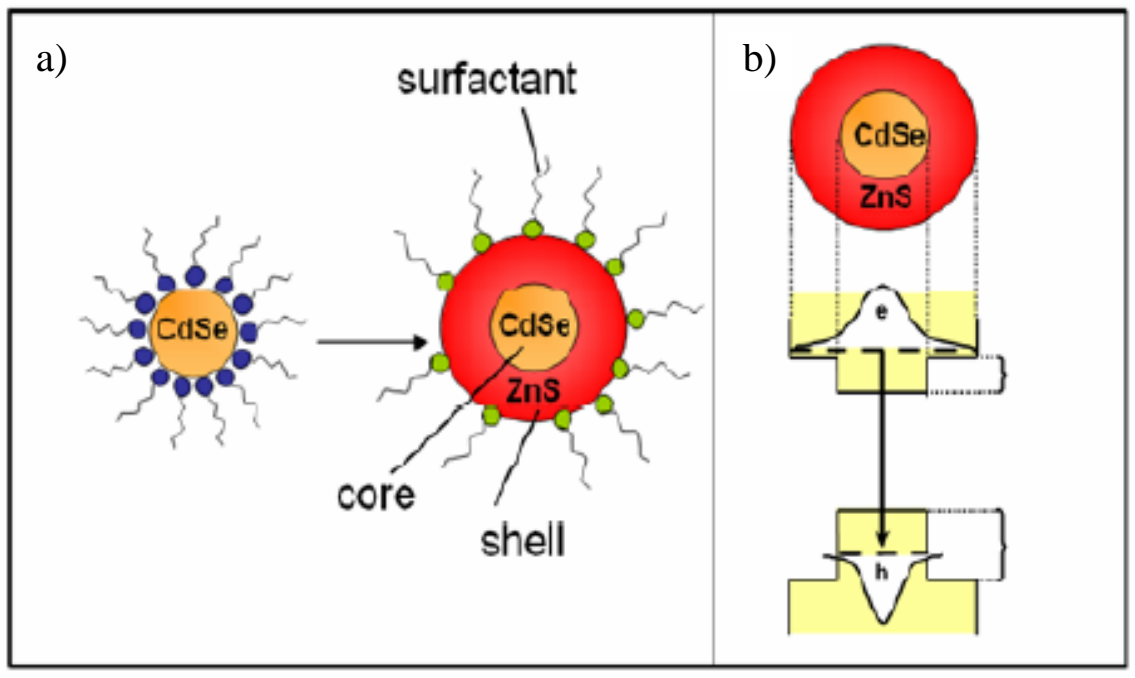

Figure 1.7 Structure and energy diagram of CdSe/ZnS core/shell QDs 
a) Structural comparision of CdSe core QDs and CdSe/ZnS core/shell QDs. b) Energy bandgap alignment of CdSe/ZnS core/shell QDs. The radial probability functions for the lowest energy electron and hole wave functions are schematic shown.

In 1996, a powerful effective chemical synthesis route for ZnS shell growth on CdSe core QDs was developed by Dr. Guyot-Sionnest and co-workers. ${ }^{[27]}$ They synthesized CdSe/ZnS core/shell QDs using organometallic precursors by a two-step single-flask method. The asprepared CdSe/ZnS core/shell QDs exhibited strong and stable band-edge luminescence with a 50\% QY at room temperature. Since then, the synthetic approach was widespreadly applied to other core/shell QDs systems, including core/shell and core/multishell QDs based on II-VI, IVVI, and III-V semiconductors. ${ }^{[26]}$ Core/shell systems are mostly prepared in a three step process: (i) initial synthesis of core QDs; (ii) isolation and purification of the core QDs; and (iii) the subsequent shell growth. During the final procedure, several monolayers (usually 1-5) of the shell material are epitaxially grown around the core QDs. In order to prevent nucleation of the shell material and uncontrolled ripening of the core QDs, the temperature for shell growth should be lower than the temperature used for the synthesis of core QDs. In addition, the shell precursors should be slowly added to keep the rate of precursor addition lower than the rate of deposition of the shell precursor onto the core QDs. In this manner, the shell precursor concentration will remain below the threshold for nucleation, eliminating possible homogeneous nucleation of the shell material in solution.

Recently, successive ion layer adsorption and reaction (SILAR) originally developed for chemical-bath deposition of thin films on solid substrates was adopted by Dr. Peng's group to grow high-quality CdSe/CdS core/shell QDs with QYs above 50\%. ${ }^{[28]}$ The SILAR technique is based on the formation of one monolayer at a time by alternating the injections of cationic and anionic precursors. Monodispersity was maintained for CdS shell thicknesses up to 5 monolayers, as indicated by the narrow PL line width ( $25 \mathrm{~nm}$ in FWHM) (Figure 1.8). Compared with the previous protocol using organometallic precursors for shell growth, the 
SILAR technique has two major advantages: (1) it employs cheaper, less toxic, and less active compounds, such as zinc carboxylates and elemental sulfur, to replace pyrophoric and highly toxic compounds (pyrophoric diethylzinc and toxic hexamethyldisilathiane). So, the SILAR technique is highly suitable for producing large-scale environmentally-benign core/shell QDs at a low price. (2) The SILAR technique precisely controls the shell growth procedure at the level of one monolayer. Complex core/shell QDs can thus be easily designed and synthesized, such as $\mathrm{CdSe} / \mathrm{CdS} / \mathrm{ZnS}^{[29]}$, and $\mathrm{CdSe} / \mathrm{CdS} / \mathrm{Zn}_{\mathrm{x}} \mathrm{Cd}_{1-\mathrm{x}} \mathrm{S} / \mathrm{ZnS}^{[30]}$ core/multishell QDs. Due to these benefits, we will employ SILAR technique to design and synthesize our CdSe-based core/shell QDs.
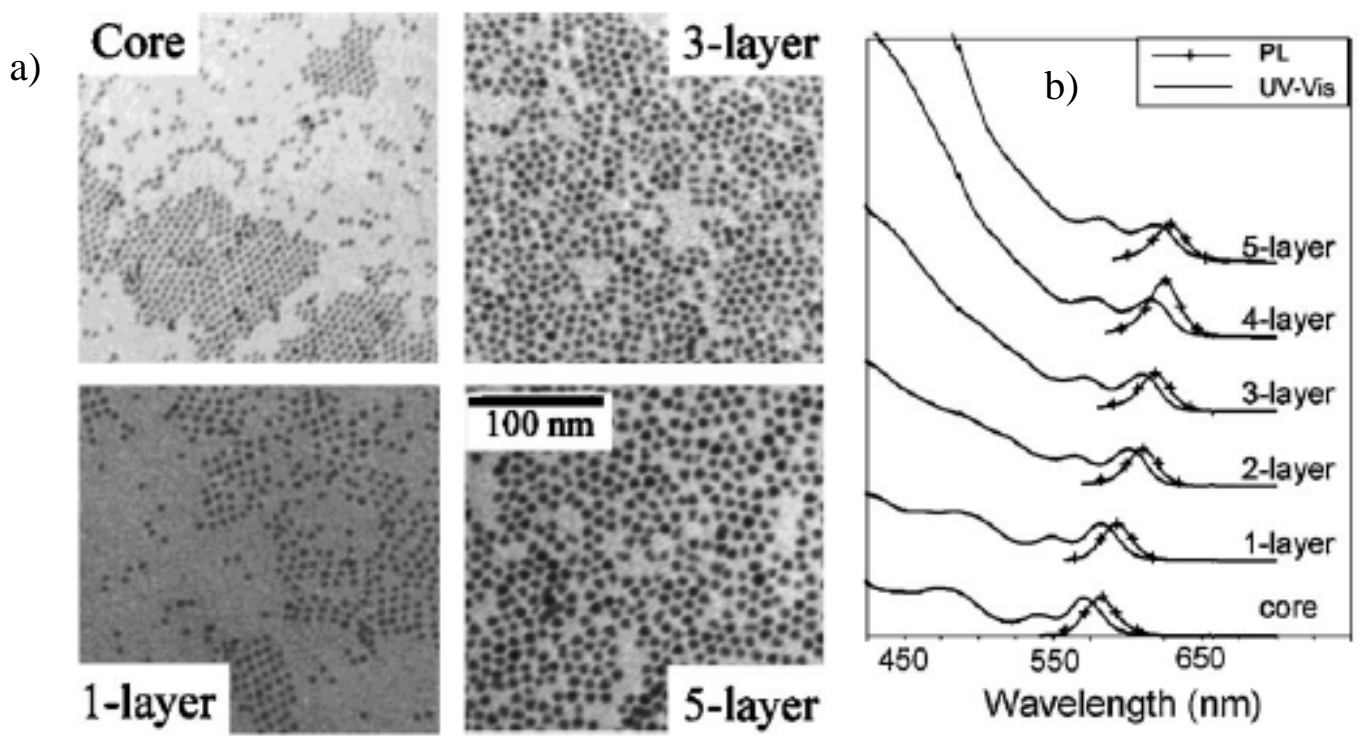

Figure 1.8 CdSe/CdS core/shell QDs synthesized by SILAR technique

a) TEM images of CdSe QDs depicting the increase in diameter upon growth of several monolayers of a CdS shell by means of the SILAR technique. b) Absorption and PL spectra of samples with different shell thicknesses.

\subsection{Colloidal QDs for Energy Conversions}

The unique properties of colloidal QDs, including highly tunable luminescence across visible and near-infrared range, saturated emission color, high luminescence QYs, and efficient light absorption, make them very attractive for developing novel energy conversion devices. ${ }^{[16]}$ Colloidal QDs also offer opportunities for inexpensive device fabrication by solution processing, 
and are thus a promising class of materials for low-cost and large-area manufacturing, such as thin film solar cells and large-area displays. ${ }^{[16]}$ Nevertheless, as discussed in previous section, colloidal QDs are capped with a layer of bulky insulating ligands. In a thin film device, the bulky surface ligands prevent interaction between QDs and consequently result in a significant energy barrier for charge injection, transport, or recombination. Hence, it is necessary to design the surface ligands of QDs to improve the charge carrier mobility. In the following three sections, a literature review is made, summarizing previous work done by other research groups on the design of optimal surface ligands of colloidal QDs and device structures for efficient energy conversions. In particularly, recent progress on the development of QD-based LEDs and solar cells is presented.

\subsubsection{Design of Surface Ligands of QDs for Energy Conversions}

\subsubsection{Original Long-Chain Organic Ligands}

As discussed in section 1.1.3, the long-chain ligands introduced during the synthesis of colloidal QDs play an important role. They control nucleation, growth, surface chemistry, and QD stability. These long-chain ligands are typically organic hydrocarbon molecules with a functional head attached to the QD surface and a long hydrocarbon chain tail interacting with the solvent. Typical long-chain ligands include OA, TOPO, octadecylamine (ODA), and HDA, as listed in Table 1.1. Due to the nonpolar nature of the ligands, QDs can be dispersed in nonpolar solvents such as hexane, chloroform, and toluene.

It is necessary to extract and purify colloidal QDs from their growth solutions, as there exist many of synthetic by-products in the solutions, such as unreacted precursors and excessive long-chain molecules. The surface ligands provide a repulsive force in solution to counteract the van der Waals force between QDs, which keeps QDs from aggregation. This repulsive force is, however, strongly dependent on the interaction between the capping ligands and the solvent. When a nonsolvent (typically acetone, or methanol), miscible with the solvent (typically hexane, 
toluene, or crucial growth solution) in which the QDs are dispersed, is added, the colloidal QDs suspension is destabilized, leading to QDs aggregation and precipitation. The precipitated QDs are then centrifuged, leaving unreacted precursors and excess ligands in the solution. The purified QDs can be redispersed in a variety of non-polar solvents, and may experience additional precipitation/redispersion cycles. Zhao and co-workers have found that a critical step to make efficient QD-LEDs was to thoroughly wash the as-prepared QDs to remove excess ligands and synthetic by-products. In their work, CdSe/CdS QDs were washed twice by dispersion in chloroform and subsequent precipitation with acetone. Although the QY of the QDs was reduced from $\sim 50 \%$ to $30 \%$ due to the removal of some surface ligands, the performance of QD-LEDs was enhanced largely due to the removal of excess ligands, which leads to improved charge injection. ${ }^{[31]}$

Another purification method for QDs was proposed by Peng and co-workers. ${ }^{[32]}$ In this process, QDs are not precipitated out of the solution. The purification of QDs is performed by repeated extraction (twice for most cases) of the reaction mixtures dissolved in hexane. An equal volume mixture of chloroform/methanol (1:1) is used as the extraction solvent. After extraction, QDs are extracted into the upper chloroform/hexane phase. Meanwhile the unreacted precursors and synthetic by-products are dissolved in the lower methanol phase. Experimental results revealed that this purification method could yield core/shell QDs of better high-quality QD than QDs purified by precipitation/redispersion. ${ }^{[28]}$ It is plausible that mild purification of QDs without precipitation can better preserve the QD surface and minimize the influence of water or oxygen. 


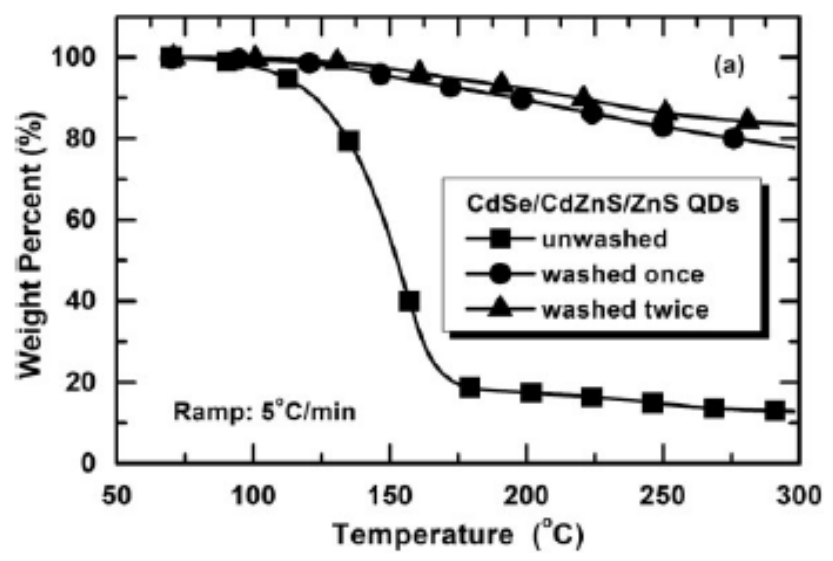

Figure 1.9 Thermo-gravitational (TGA) analysis of ODA-capped CdSe/CdZnS/ZnS core/shell/shell QDs

The TGA results demonstrate that the existence of excess amount of ligands on the QDs before precipitation/redispersion washing and the weigh-loss caused by the ligands desorbing from the QDs washed 1 and 2 times. $^{[33]}$

Although the insulating-chain ligands impede the injection and transport of charge carriers, they have proven to be the most efficient ones to passivate the surface states of QDs. QD-LEDs fabricated from QDs with long-chain ligands have achieved the best performance so far. ${ }^{[15]}$ Thermal annealing of QDs has been verified as an effective way to partially remove surface ligands (Figure 1.9). In 2007, Niu and co-workers reported improved performance of QD-LEDs via thermal annealing of the QD layer. ${ }^{[33]}$ In-situ annealing at $180{ }^{\circ} \mathrm{C}$ of the CdSe/CdZnS/ZnS core/shell/shell QDs active layer, prior to the final deposition of the organic electron transport layer, enhanced the external quantum efficiency (EQE) by $\sim 3$ times and the luminous power efficiency by $\sim 4$ times compared to identical QD-LEDs without thermal annealing. Because $180{ }^{\circ} \mathrm{C}$ is well above the "melting transition" temperature of ODA chains on the QD surfaces, the annealing could result in increased mobility of the QDs and reduce the inter-QD spacing in the device. In addition, ODA desorption during annealing at $180{ }^{\circ} \mathrm{C}$ could improve charge injection and energy transfer from the organic semiconductors to the QDs by decreasing the thickness of the insulating ligand shell surrounding each QD. 
Table 1.1 Different type of surface ligands for colloidal QDs

\begin{tabular}{|c|c|c|c|}
\hline Ligand Type & Molecule Structure & $\begin{array}{c}\text { Interparticle } \\
\text { distance }\end{array}$ & Properties \\
\hline $\begin{array}{l}\text { Original } \\
\text { long-chain } \\
\text { molecules }\end{array}$ & $=-\mathrm{HS},-\mathrm{COOH},-\mathrm{NH}_{2}, \mathrm{OP} \leftarrow$, etc. & $>1.5 \mathrm{~nm}$ & $\begin{array}{l}>\text { Regular ligands employed for QD } \\
\text { synthesis } \\
\text { > Colloidal solution is highly stable in } \\
\text { nonpolar solvent } \\
\text { > Hydrophobic surface } \\
>\text { High PL QYs (>50\%) } \\
\text { > Highly insulating QD film } \\
>\text { Ideal for light emission applications }\end{array}$ \\
\hline $\begin{array}{c}\text { Short-chain } \\
\text { molecules }\end{array}$ & $\begin{array}{l}\text { Two functional heads: } \\
\mathrm{QD}=-\mathrm{HS},-\mathrm{COOH} \text {, or }-\mathrm{NH}_{2}\end{array}$ & $0.3-1 \mathrm{~nm}$ & $\begin{array}{l}>\text { Ligand exchange in solution or film } \\
\text { level } \\
>\text { Colloidal solution is stable in polar } \\
\text { solvent } \\
>\text { Improved charge transport } \\
>\text { Hydrophilic surface } \\
>\text { Low PL QYs (<30\%) } \\
>\text { Ideal for solar cell and } \\
\text { photodetector applications }\end{array}$ \\
\hline $\begin{array}{c}\text { Inorganic } \\
\text { ligands }\end{array}$ & $=\mathrm{S}^{2-}, \mathrm{HS}^{-}, \mathrm{NH}_{2}^{-}, \mathrm{SnS}_{4}{ }^{4-}, \operatorname{In}_{2} \mathrm{Se}_{4}{ }^{2-}$ etc. & $\sim 0.5 \mathrm{~nm}$ & $\begin{array}{l}\text { Digand exchange in solution } \\
\text { > Colloidal solution is stable in polar } \\
\text { solvent } \\
>\text { Improved charge transport } \\
>\text { Hydrophilic surface } \\
>\text { High electron mobility }\end{array}$ \\
\hline
\end{tabular}




\subsubsection{Short-Chain Organic Ligands}

As discussed in previous sections, long-chain organic ligands produce a large insulating barrier for charge transport in QD film, and thus limit the applicability of QDs to optoelectronic devices. Researchers have been searching for alternative ligands which can reduce the surface energy barrier while retain good passivation of the QDs. Exchange of the original long-chain molecules with short-chain ones (Table 1.1) is a widely used approach and has been employed to improve the charge transport in QD-based LEDs, solar cells, photodetectors, and field-effect transistors (FETs).

The ligand exchange process typically consists of repeated exposure of the QDs with long-chain ligands to an excess of competing ligands, leading to partial or complete exchange of surface molecules. The exchange process is often facilitated by a two-phase mixture of nonpolar and polar solvents to separate the QDs, accelerated by vigorously stirring as well as gentle heating if necessary. ${ }^{[34]}$ In addition, the ligand exchange can also be applied to QD film. Two different processing routes can be used for ligand exchange of QDs on the film level: (1) The ligand exchange can be conducted via soaking of a spin-coated QD film in a solution containing shorter-chain ligands. ${ }^{[35-36]}$ However, the rate of ligand exchange is limited by slow ligand diffusion through the film. This soaking technique may also lead to film cracks as a shorter interparticle distance reduces the volume of the film. ${ }^{[37]}$ (2) Large area and crack-free QD films can be achieved by the layer-by-layer (LBL) deposition technique. ${ }^{[37-38]}$ In a LBL process, the substrate is dipped successively in a long-chain-ligand capped QD solution and a solution of short-chain molecules with two functional heads that can bind to two neighboring QDs. This procedure is typically repeated many times to build up a QD layer with an ideal thickness.

\section{(i) Exchange with Short-Chain Organic Ligands with Single Functional Head}

The first successful solution-based ligand exchange was conducted via pyridine for CdSe QDs. ${ }^{[2]}$ It was done by refluxing QDs in pyridine, resulting in QDs insoluble in nonpolar solvents, but readily dispersible in polar solvents. Pyridine can also be removed by mild heat treatment or 
vacuum. It was found that the pyridine treatment could significantly improve the charge transport and photoconductivity for $\mathrm{CdS}^{[39]}$ and $\mathrm{PbSe}^{[40]}$ QDs solids. Furthermore, the first prototype allinorganic QD solar cells were processed from pyridine-capped CdSe and CdTe quantum rods solution, and demonstrated power conversion efficiency $~ 3 \%$ and impressive stability in air (Figure 1.17). ${ }^{[12]}$ Another widely used short-chain molecule with single functional head is butylamine. Highly-sensitive solution-processed photodetectors with butylamine-capped $\mathrm{PbS}^{[41]}$ or CdSe ${ }^{[13]}$ QD film as the active layer were reported. Similar work has been done at the film level, OA-capped PbS or oleylamine-capped CdSe QDs in non-polar solvents were spin-coated onto a gold or ITO substrate to form a solid-state film with an ideal thickness. The film was then treated in a mixture of butylamine in acetonitrile for enough long time (typically over two days). The devices based on butylamine-capped QDs demonstrated an increased exciton dissociation efficiency and improved charge transport properties. The third signature ligand is p-phenylene baring a binding functionality $(\mathrm{X})$ and a variable polar terminal group $(\mathrm{Y})$ (Figure 1.10). SoreniHarari and co-workers demonstrated that the InAs QD energy levels can be tuned using surface ligand exchange. ${ }^{[42]}$ Based on the current-voltage characterization of single layer devices consisting of a film of InAs QDs sandwiched between two electrodes, the p-phenylene-capped QDs film supported an order of magnitude higher current than a TOP-capped QD film, due to the improved charge transport after ligand exchange. Also, compared to the type I alignment between poly(p-phenylene vinylene) (PPV) and TOP-capped InAs QDs, a type II band alignment between PPV and p-phenylene-capped QDs can be formed due to the HOMO-LUMO levels downward shift of p-phenylene-capped QDs. To demonstrate how this ligand-induced shifts can be used to engineer device performance, they fabricated bilayer solar cells composed of ITO/poly(3, 4-ethylenedioxythiophene) (PEDOT)/PPV/InAs QDs/Ag. The photocurrent spectral response of the device based on the p-phenylene-capped QDs was two orders of magnitude higher than that based on the TOP-capped QDs. These results predict that the QD surface ligands manipulation would allow tuning of the energy level alignment of QDs-polymer systems to 
achieve better performance of solar cells or LEDs. However, the short-chain ligands do have some drawbacks: (i) the QDs passivated by short-chain ligands exhibit extremely low PL QY due to weak passivation of the QD surface defects; ${ }^{[34,43]}$ (ii) these short-chain ligands are not stable against oxidation and volatility; (iii) the QD colloidal stability with the shot-chain ligands is decreasing, ${ }^{[15]}$ resulting to QDs aggregation if external agitation is applied, such as heat or ultrasound treatment.

(a)

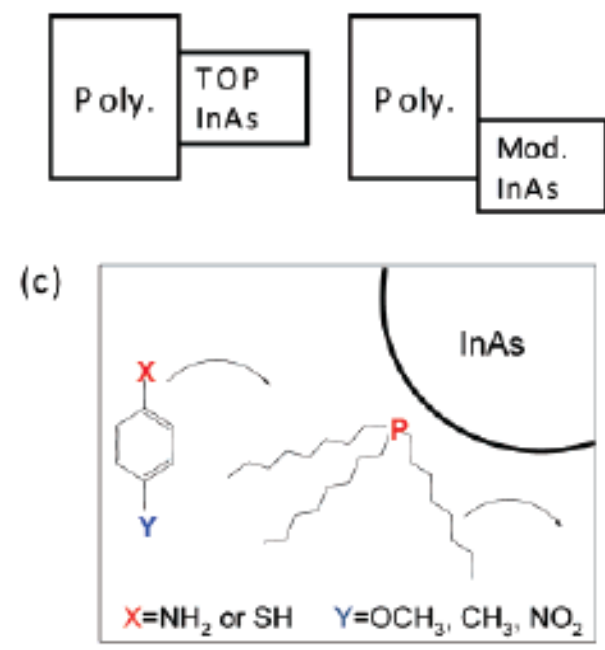

(d)

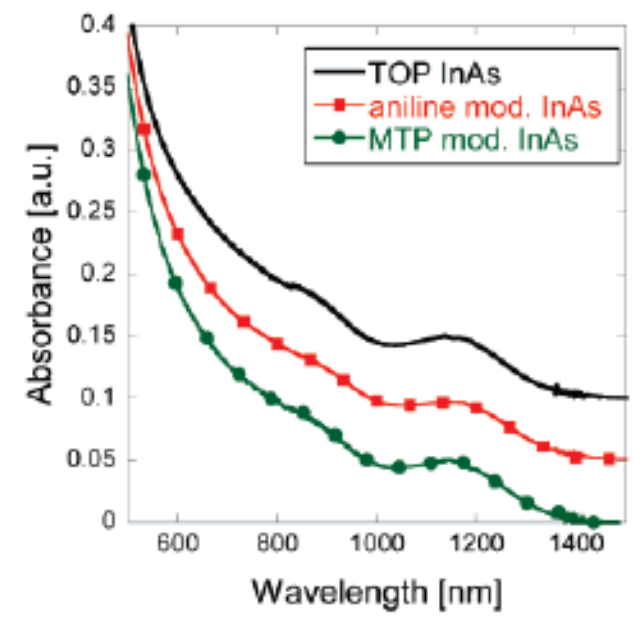

Figure 1.10 Energy levels tuning of InAs QDs through ligand exchange

Schematic energy level diagram of the type I (a) and type II (b) alignment. (c) Modification of NC surface properties. Ligand exchange of TOP-capped InAs QDs with a p-phenylene ligand. (d) Absorbance spectra of TOP-capped InAs QDs in toluene (solid) in comparison to its surface-modified aniline (squares) and MTP (circles) QDs in tetrahydrofuran. ${ }^{[42]}$

\section{(ii) Exchange with Short-Chain Organic Ligands with Two Functional Heads}

Cross-linking of QDs via short-chain ligands with two functional heads provides an alternative way to reduce the interparticle distance and improve charge transport between QDs. In colloidal solutions, the cross-linking of QDs by these ligands would result in QDs precipitation. Therefore, this kind of ligand exchange is typically conducted under the film level, and used for fabrication of various optoelectronic devices of QDs. ${ }^{[38,44]}$ Talgorn and co-workers prepared highly photoconductive films of CdSe QDs by exchanging the original bulky ligands 
with 1, 2-ethanedithiol (EDT) and 1, 2-ethanediamine (EDA), and compared two different film fabrication methods (LBL deposition and soaking of spin-coated films). ${ }^{[45]}$ They found that the LBL method was superior to the soaking approach. The LBL films exhibit stronger electronic coupling, less disorder, and a higher photoconductivity, but their PL was fully quenched.

Luther and co-workers demonstrated efficient and stable colloidal QDs Schottky solar cells fabricated using the LBL technique with EDT as the cross-linking ligand. ${ }^{[37]}$ In their LBL process, a monolayer of QDs was deposited onto a patterned ITO substrate via dip coating from a hexane solution and then washed in $0.01 \mathrm{~mol} / \mathrm{L}$ EDT in acetonitrile to remove the bulky insulating OA ligands. 25-40 dip coating cycles would build films up to 60-300 nm thick. PbSe QD solar cells exhibited an EQE of 55-65\% in the visible range and up to 25\% in the infrared region, with a spectrally corrected AM1.5G power conversion efficiency of $2.1 \%$. They also demonstrated solar cells fabricated from PbS and CdSe QDs, indicating that the LBL method is not restricted to EDT-treated PbSe QDs.

The cross-linking ligand exchange strategy can be also applied to fabricate QD-LEDs and improve their performance. Cho and co-workers demonstrated that crosslinking a QD layer via 1, 7-diaminoheptane small molecules could shift the valence band of the QDs upward, thus reducing the band offset with the hole transport layer (HTL) and substantially improving the charge injection in the QD-LEDs. ${ }^{[44]}$ Additionally, thermal annealing of the crosslinked QD layer further improved device luminance and luminous efficiency due to a decrease in the contact resistance through the removal of organic residues in the QD layer. The resulting device exhibited a high luminance of $12,380 \mathrm{~cd} / \mathrm{m}^{2}$, low turn-on voltage of $1.9 \mathrm{~V}$, and high power efficiency of $2.41 \mathrm{~lm} / \mathrm{W}$.

\subsubsection{Inorganic Ligands}

A recent approach towards better electronic coupling between QDs was developed by Dr. Talapin's group to replace the bulky organic ligands of QDs with molecular metal chalcogenide complexes (MCCs) inorganic ligands. ${ }^{[46]}$ In a typical exchange process, long-chain organic- 
ligands-capped QDs in nonpolar solvent (for instance, hexane or toluene) were mixed with a solution of MCCs in hydrazine or dimethylsulfoxide, and vigorously stirring was performed to enable the ligand exchange and phase transfer of the MCC-capped QDs from the nonpolar solution into the polar one.

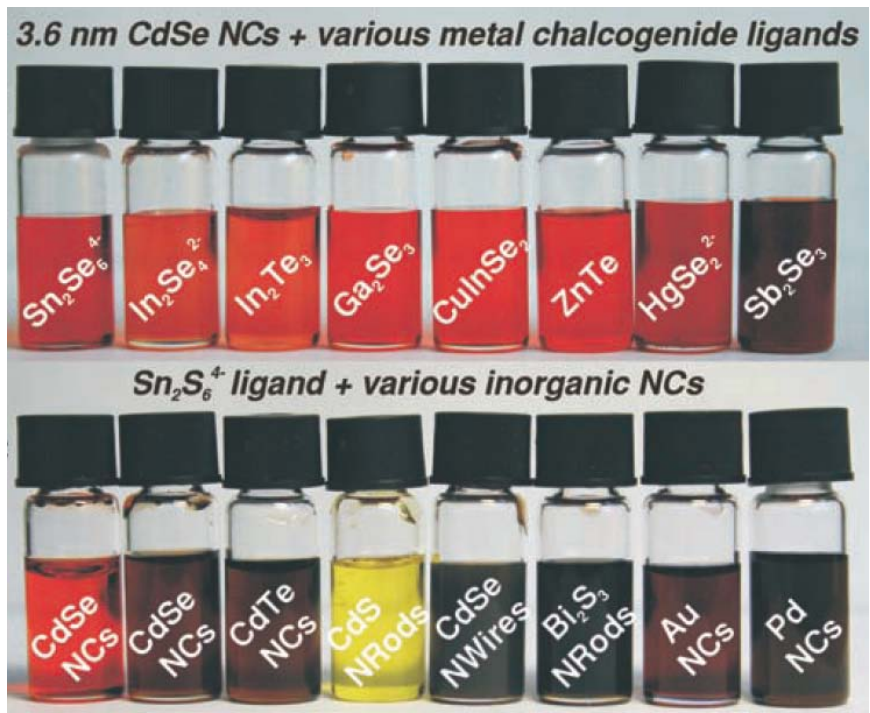

Figure 1.11 Stable colloidal solutions of MCC-capped QDs

Solutions of CdSe QDs capped with various MCC in hydrazine, and of eight different types of QDs combined with the $\mathrm{Sn}_{2} \mathrm{~S}_{6}{ }^{4-}$ ligand (NCs=nanocrystals, NRods=nanorods, NWires=nanowires; the color difference between the first two CdSe NC solutions is due to the different particle diameter: 3.6 and $5.8 \mathrm{~nm}$, respectively). ${ }^{[46]}$

The generality of this approach has been validated. As illustrated in Figure 1.11, 3.6 nmCdSe QDs can be capped with various MCCs, and various QDs with different shape and size can be capped with $\mathrm{Sn}_{2} \mathrm{~S}_{6}{ }^{4-}$ ligand, leading to stable colloidal solutions. High colloidal stability is achieved due to the electrostatic interaction provided by the two terminal negatively charged sulfur atoms. Unfortunately, QDs could not retain their superior optical properties when the ligand exchange is performed in some harsh solvents like hydrazine. The same group has found that similar ligand exchange can be conducted in a more benign aqueous solution, which can help to largely preserve the high PL QYs of QDs. ${ }^{[47]}$ Recently, they further studied the ligand exchange of QDs with a variety of metal-free inorganic ligands, including $\mathrm{S}^{2-}, \mathrm{HS}^{-}, \mathrm{OH}^{-}$, etc. ${ }^{[48]}$ All these inorganic ligands are much smaller than organic ligands, and thus greatly reduce the 
inter-QD spacing in closely-packed QD films.

Electronic devices based on inorganically-capped QDs have been demonstrated through prototype photodetectors and FETs. ${ }^{[49]}$ FETs using $\mathrm{In}_{2} \mathrm{Se}_{4}{ }^{2-}$-capped CdSe QDs exhibited bandlike electron transport and high electron mobility of $16 \mathrm{~cm}^{2} \mathrm{~V}^{-1} \mathrm{~s}^{-1}$, which is about an order of magnitude higher than the best results reported solution-processed organic and QDs devices so far. The photodetectors based on layers of $\mathrm{In}_{2} \mathrm{Se}_{4}{ }^{2-}$-capped CdSe/CdS QDs, sandwiched between indium tin oxide (ITO) and aluminum electrodes, presented normalized detectivity $\mathrm{D}^{*}>1 \times 10^{13}$ Jones, which is a record for this type of devices. However, up to now, there is little work on solar cells and LEDs based on inorganically-capped QDs. One goal of this dissertation is to evaluate the applicability of CdSe QDs with MCC ligands for energy conversions in light emission and photovoltaic conversion processes.

\subsubsection{QD-based Light-Emitting Diodes}

Colloidal QDs are considered as one of the most promising emitters suitable for developing high-quality LEDs, ${ }^{[11,20,50]}$ due to their superior optical properties, such as high color purity, high luminescence QYs (>80\%), and tunability of the emission color from UV to near-IR. For a typical QD-LED, a layer of QDs is sandwiched between the HTL and electron transport layer (ETL), which provide injection of carriers into the QDs (Figure 1.12). Although the underlying device physics of QD-LEDs have been sufficiently addressed, the performance of the state of the art devices lags significantly behind inorganic and organic LEDs. Performance improvement requires better energy alignment between QDs and HTL/ETL, higher stability of HTL/ETL, better surface design of QDs, and more efficient excitonic energy transfer and direct carrier injection from HTL/ETL into the QDs. In this section, we briefly review recent progress in QD-LEDs. 


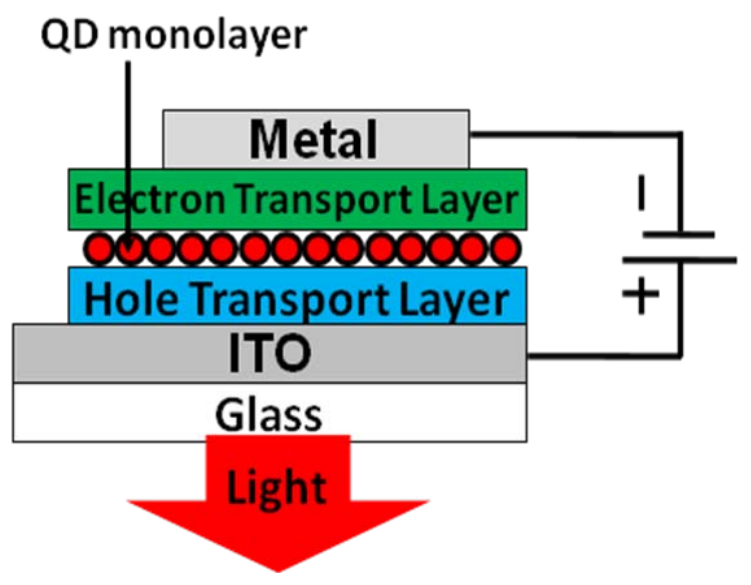

Figure 1.12 Cross-section structure of a typical QD-LED

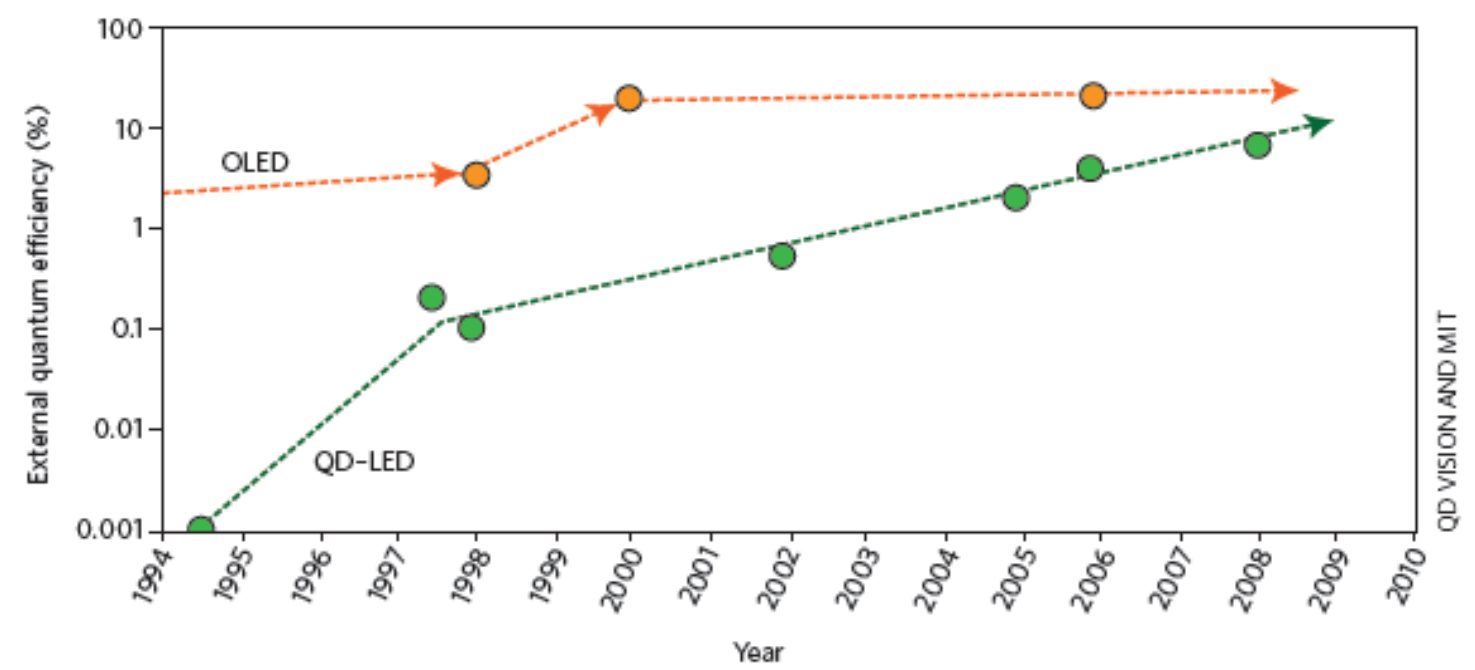

Figure 1.13 Development of QD-LEDs (green circles) and OLEDs (orange circles) over the time ${ }^{[51]}$

The first LED employing CdSe colloidal QDs was reported by the Alivisatos group in 1994. ${ }^{[52]}$ Since then, significant progress has been achieved by optimizing all the components of QD-LEDs. The EQEs of QD-LEDs has evolved from the early demonstration of $0.001 \%$ in $1994^{[52]}$ to 7\%, a result reported by QD Vision in 2009 ${ }^{[51]}$ (Figure 1.13). Using core-shell QDs as the emitters allowed one to significantly increase the efficiency of radiative recombination and device internal quantum efficiency. ${ }^{[25]}$ To achieve higher injection currents, heavily doped electron transporting layers were introduced, whereas the thickness of the emitting layer was 
reduced down to one or two monolayers of highly luminescent semiconductor QDs. ${ }^{[53]}$ One important breakthrough in understanding the operation of QD-LEDs is achieved by Bulovic's and Bawendi's groups, who have found that excitons formed in the fluorescent organic materials can be nonradiatively transferred to and recombined in the semiconductor QDs. ${ }^{[53]}$
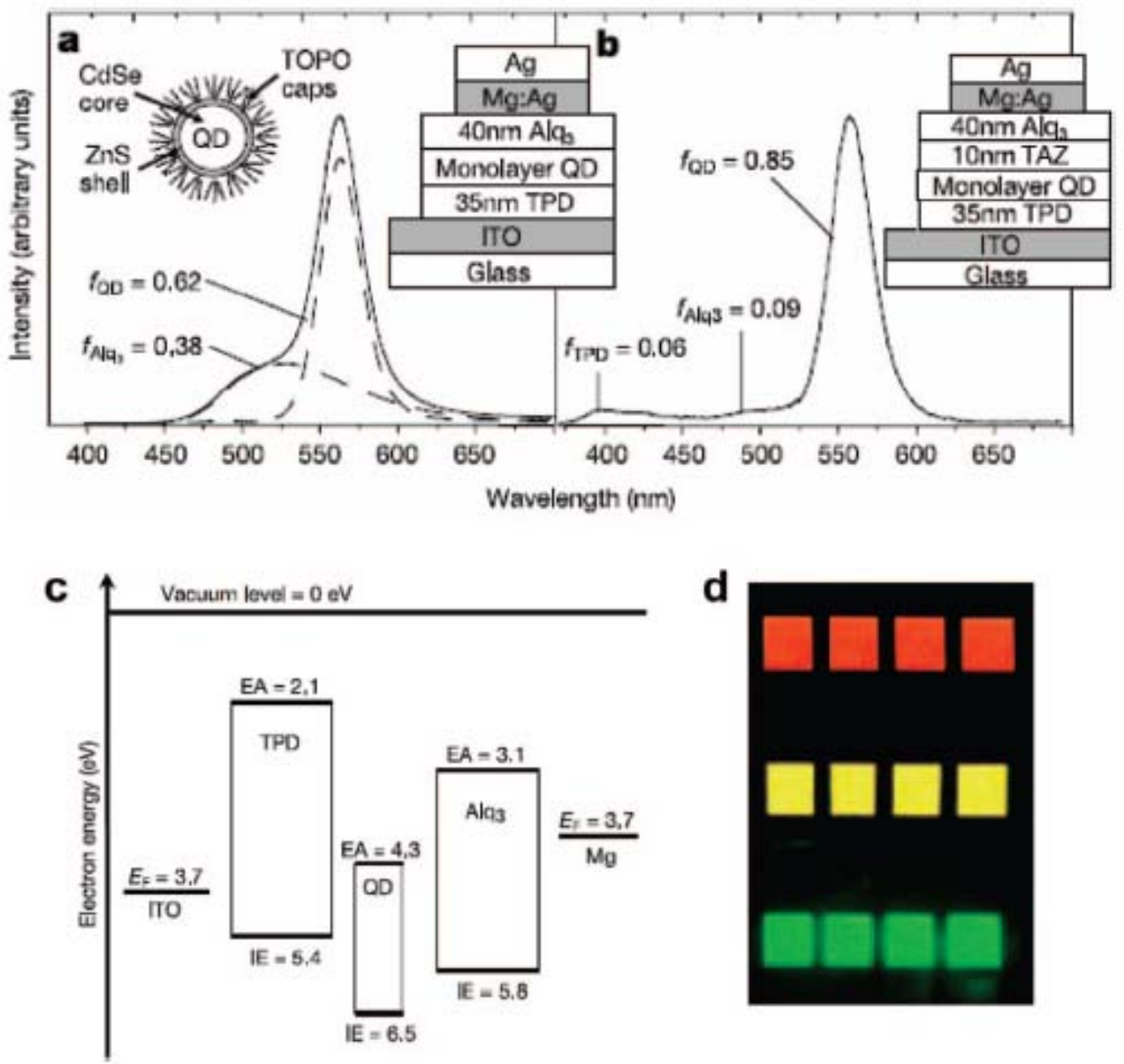

Figure 1.14 Electroluminescence spectra and structures for two QD-LEDs

(a) Without and (b) with a hole-blocking layer (TAZ). Alq $\mathrm{A}_{3}$ is tris (8-hydroxyquinoline) aluminum; TPD is $N, N^{\prime}-$ bis(4-butylphenyl)- $N, N^{\prime}$-bis(phenyl)-benzidine; TAZ is 3-(4-biphenylyl)-4-phenyl-5-t-butylphenyl-1,2,4-triazole.(c) Proposed energy diagram of the QD-LED. ${ }^{[53]}$ (d) photographs of green, yellow, and red LEDs using CdSe/ZnS core/shell QDs with different sized of CdSe cores. ${ }^{[29]}$

The structure of the most efficient QD-LEDs is shown in Figure 1.14. A single monolayer of CdSe/ZnS QDs is sandwiched between ETL and HTL. When forward bias is applied, electrons and holes are injected into the QD layers from ETL and HTL layers, 
respectively. The recombination of electron-hole pairs in the QDs generates photons with the energy corresponding to the gap between highest occupied (1Sh) and lowest unoccupied (1Se) states in the QDs. To deposit a monolayer of QDs, a novel phase-separation fabrication technique has been developed, ${ }^{[54]}$ in which QDs are spun coated from a mixture of TOPO-capped CdSe/ZnS QDs and the hole transport material TPD in chloroform. During the process, the QDs and TPD are separated, leading to the formation of a single QD monolayer on top of a $35 \mathrm{~nm}$ thick TPD film. The LEDs are finished by thermal evaporation of $10 \mathrm{~nm}$ TAZ hole blocking layer, $40 \mathrm{~nm} \mathrm{Alq}$ ETL, and Mg/Ag cathode.

In 2009, Anikeeva and co-workers reported QD-LEDs with EL tunable over the entire visible spectrum from $460 \mathrm{~nm}$ (blue) to $650 \mathrm{~nm}$ (deep red) (Figure 1.15). ${ }^{[55]}$ Utilizing the contact printing technique via parylene-C coated PDMS, a monolayer of QDs is transferred onto the top of a thermal-evaporated spiro-TPD layer. The detailed strucuture is composed of ITO/PEDOT:PSS/spiro-TPD/QDs/TPBi/Mg/Ag (Figure 1.15). By using high-QY QDs and wide-band-gap charge transport layers, the EQE of hybrid organic/QD LEDs was increased to 2.7\% for QD-LEDs emitting orange color. 

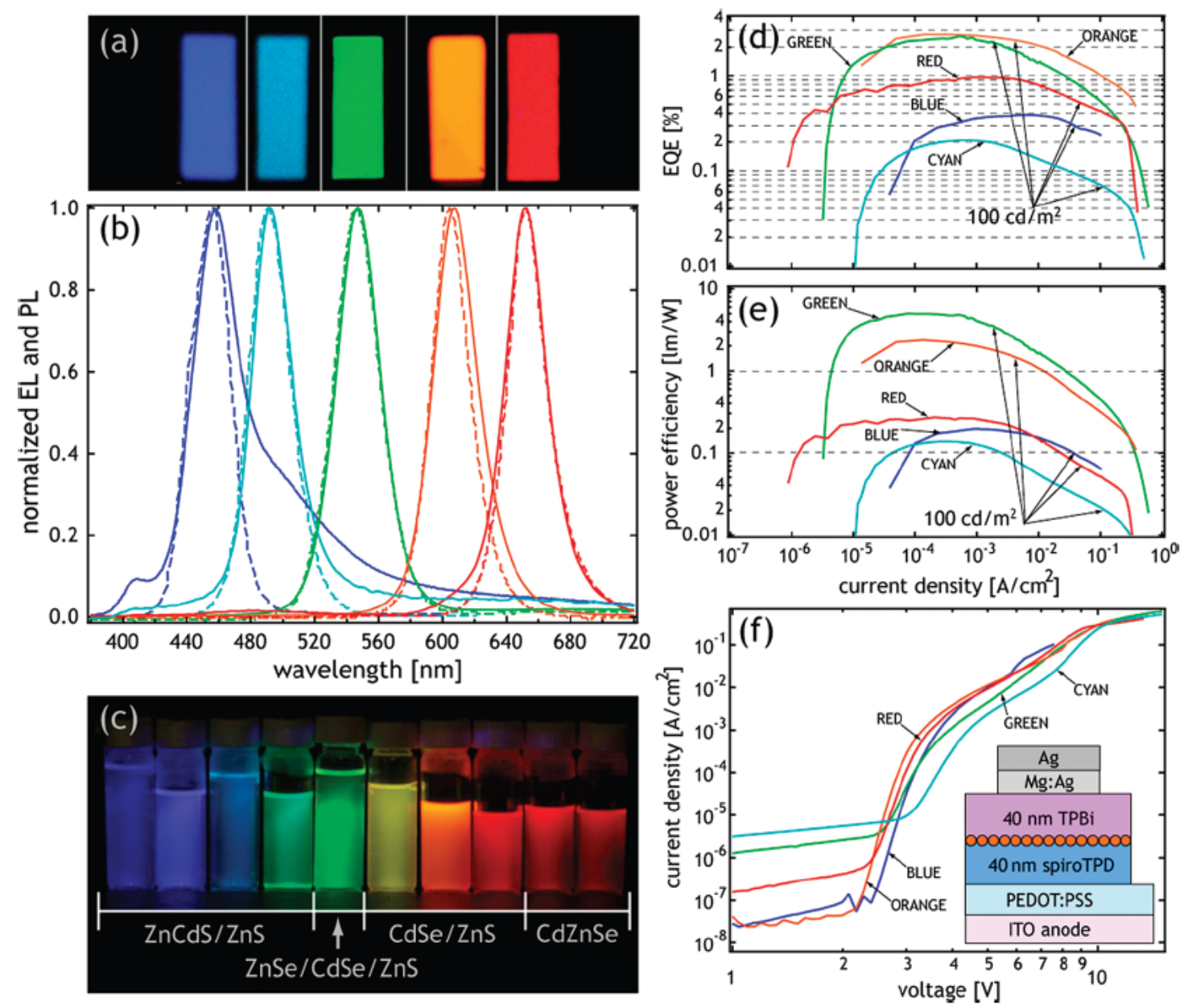

Figure 1.15 QD-LEDs with EL tunable over the entire visible spectrum from $460 \mathrm{~nm}$ (blue) to $650 \mathrm{~nm}$ (deep red)

(a) Photographs of $0.6 \times 1.9 \mathrm{~mm}^{2}$ QD-LED pixels. (b) EL spectra (solid lines) of QD-LEDs and PL spectra (dashed lines) of QD in hexane solution. (c) Photograph of the PL of chloroform solutions of different QDs. (d) EQE in \% and (e) power efficiency in $\mathrm{lm} / \mathrm{W}$ for red, orange, green, cyan, and blue QD-LEDs are plotted vs. current density. (f) I-V characteristics of the QD-LEDs. Inset shows the schematics of the device structure. ${ }^{[55]}$

Most recently, a team from the Frontier Research Laboratory at the Samsung Advanced Institute of Technology succeeded in developing the world's first full-color quantum dot display. ${ }^{[56]}$ Blue-emitting CdS/ZnS core/shell QD, green-emitting CdSeS/ZnS core/shell QD, or red-emitting CdSe/CdS/ZnS core/shell/shell QD layer was integrated into ITO/PEDOT:PSS/polymer TFB/QDs/ $\mathrm{TiO}_{2} / \mathrm{Al}$ device structure as the emitters (Figure 1.16). The wavelengths at the EL peak for the red, green and blue QD-LED were 615, 530, and $480 \mathrm{~nm}$, 
respectively. The respective maximum brightness values were $16,380,6,425$ and $423 \mathrm{~cd} / \mathrm{m}^{2}$. The maximum power efficiency of the red QD-LED was $4.25 \mathrm{~lm} / \mathrm{W}$. They have developed an elegant solvent-free transfer printing method to deposit the QDs multilayer onto the TFB HTL. The adoption of this method enabled large-scale quantum dot displays to be produced and moreover, luminous efficiency was improved as QDs can be rearranged tightly in a process of transferring quantum dots to the substrates. Similar displays were made not only on glass but also on plastic substrates, predicting well for producing flexible QD displays in the future.

(a)

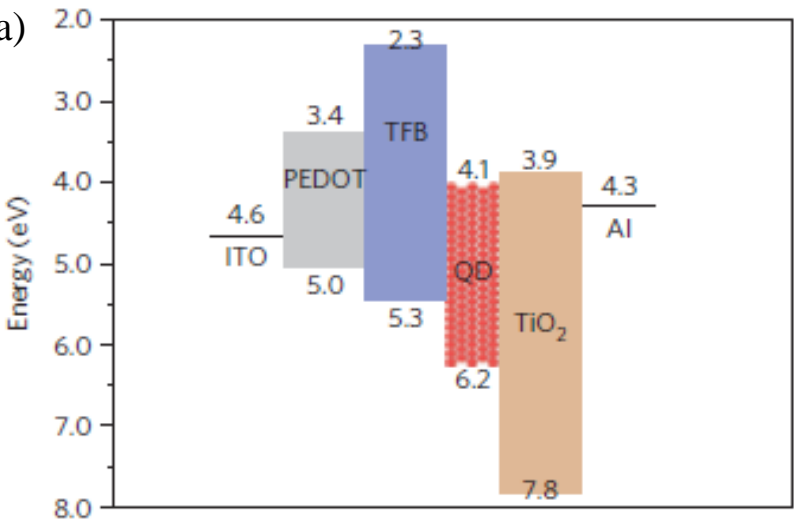

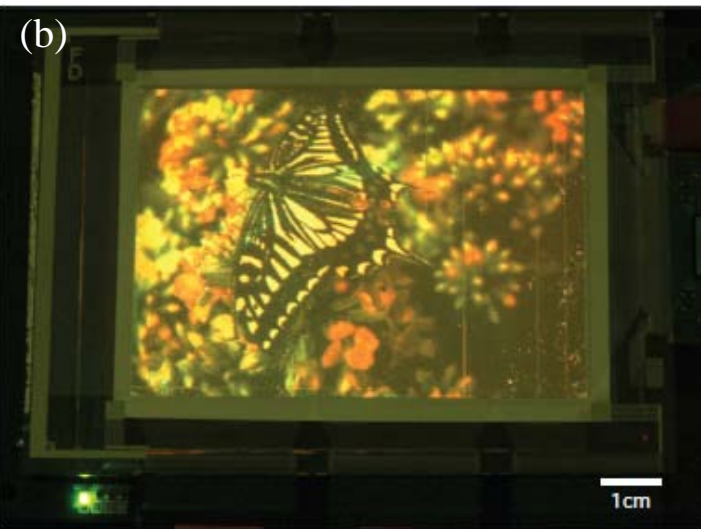

Figure 1.16 Energy diagram of QD-LEDs and full-color QD display

(a) Energy band diagram of QD-LEDs using $\mathrm{TiO}_{2}$ and polymer TFB as the ETL and HTL, as well as quantitative energy levels. (b) Electroluminescence image of a 4-inch full-color QD display using a HIZO TFT backplane with a $320 \times 240$ pixel array. ${ }^{[56]}$

Currently, further optimization of ETL and HTL layers and improvement of highlyquality semiconductor QDs are the active research topics. Generally speaking, core/shell QDs have an advantage for QD-LEDs due to their enhanced PL and EL quantum efficiencies and their greater tolerance to the processing conditions during device fabrication.

\subsubsection{QD-based Solar Cells}

The growing demand for renewable energy requires significant efforts to be invested in 
the development of efficient and inexpensive photovoltaic materials. Semiconductor QDs are considered as a promising candidate for photovoltaic applications due to the combination of superior optical and electronic properties of inorganic semiconductors with the opportunities for inexpensive, solution-based device fabrication. QD-based photovoltaics have undergone considerable progress over the recent years and are expected to make a significant technological impact in the near future.

Photovoltaic cell contains materials that absorb light and separate photogenerated electron and holes. There are different types of solar cell structure which enable efficient light absorption and separation of positive and negative charge carriers, including planar p-n junctions, donor-acceptor heterojunctions, Schottky barrier diodes, and semiconductor-liquid interfaces. Most of these structures have been considered for QD-based devices. Here, we mainly review the recent work on solar cells based on nanocrystal thin film structures, which have yielded the best conversion efficiency so far.
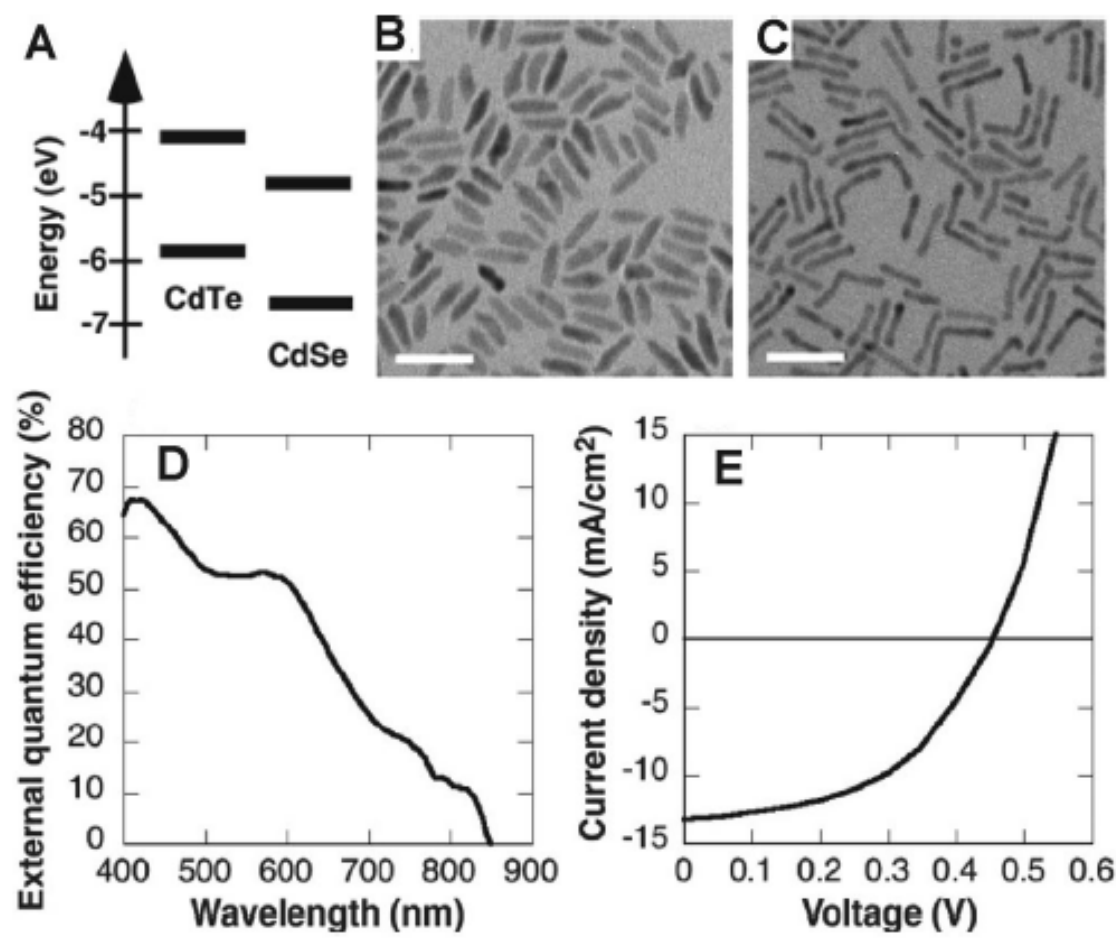

Figure 1.17 All inorganic CdTe/CdSe QD solar cell

(A) An energy diagram of the conduction and valence band levels in the CdTe/CdSe system. (B, C) TEM images of CdSe and CdTe nanorods, respectively. Scale bars are $40 \mathrm{~nm}$. (D) Incident photon to current efficiency spectrum for 
a sintered device composed of the layers of CdSe and CdTe nanorods. (E) I-V characteristic for the device exhibiting 2.9\% power conversion efficiency under AM1.5 illumination. ${ }^{[12]}$

All-inorganic solar cells made of colloidal nanocrystals (including QDs) could avoid unstable organic components while keeping the benefits of solution-based device fabrication. For example, CdSe and CdTe nanorods can create a donor-acceptor heterojunction with a staggered band alignment (Figure 1.17 a). Gur and co-workers fabricated a photovoltaic cell with spincoated layers of pyridine-treated CdTe and CdSe nanorods (Figure $1.17 \mathrm{~b}$ and c). ${ }^{[12]}$ Under illumination, photogenerated electrons transferred to the CdSe phase, while holes found lower energy states in the CdTe phase. The difference in work functions of ITO and Al electrodes provided additional driving force for charge separation. Sintering of the nanorods significantly improved carrier mobility and the device performance. With a short-circuit current $=13.2$ $\mathrm{mA} / \mathrm{cm}^{2}$, an open-circuit voltage $=0.45 \mathrm{~V}$, and a fill factor $=0.49$, solution-processed solar cells demonstrated a power conversion efficiency of 3\% and impressive stability in air (Figure $1.17 \mathrm{~d}$ and e). In a similar device configuration with $\mathrm{Cu}_{2} \mathrm{~S}$ QDs and $\mathrm{CdS}$ nanorods, Wu et al. demonstrated a solar conversion efficiency of $1.6 \% .{ }^{[57]}$ 

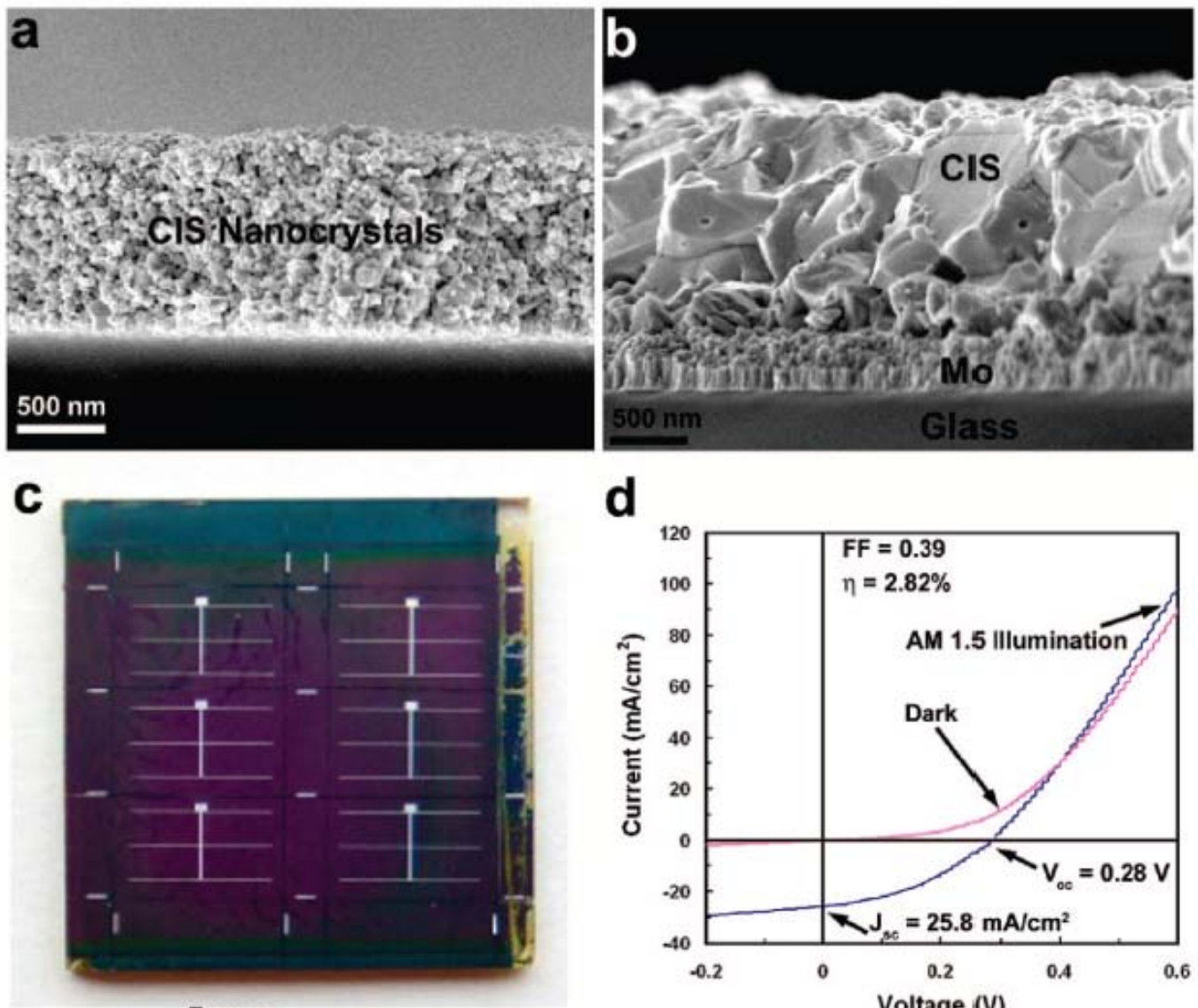

$5 \mathrm{~mm}$

d

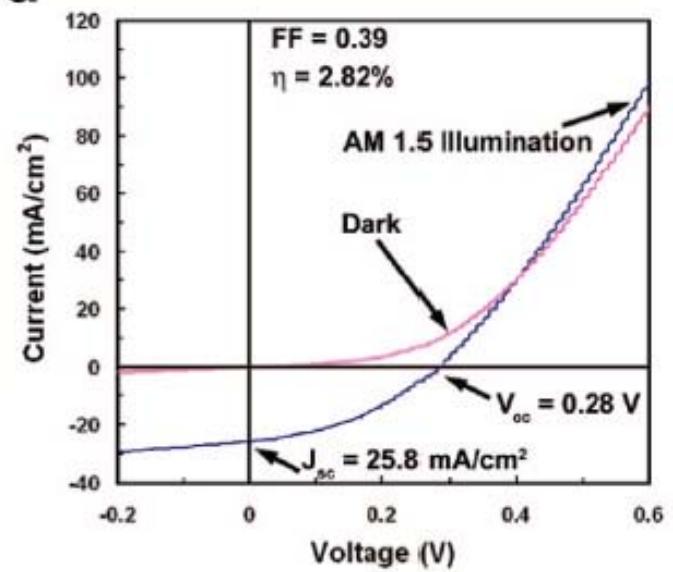

Figure 1.18 CuInSe 2 QD Solar cells

(a) Cross-section scanning electron microscopy (SEM) image of $\sim 1.5 \mu \mathrm{m}$ thick active layer spin-coated from $\mathrm{CuInSe}_{2}$ nanocrystal ink. (b) Same film after annealing under Se vapors forms large CIS crystallites. (c) A photograph and (d) solar cell characteristics for the complete device.

Bulk I-III-VI $\mathrm{V}_{2}$ chalcopyrite compounds are known as efficient photovoltaic materials. Solar cells based on copper indium-gallium selenide (CIGS) routinely demonstrate $>15 \%$ solar conversion efficiencies and are used to fabricate a wide range of commercial products. Furthermore, CIGS has good environmental prospects because it is free of highly toxic elements. Recently, colloidal synthesis of $\mathrm{CuInS}_{2}$, CuInSe 2 , and CIGS nanocrystals was reported. ${ }^{[58]}$ These photovoltaic conversions of these nanocrystals as the absorber layer have also been tested in prototype solar cells based on a type-II heterojunction. Efficiencies of $\sim 3 \%$ were achieved using sintered CuInSe 2 nanocrystals (Figure 1.18). ${ }^{[59]}$ 

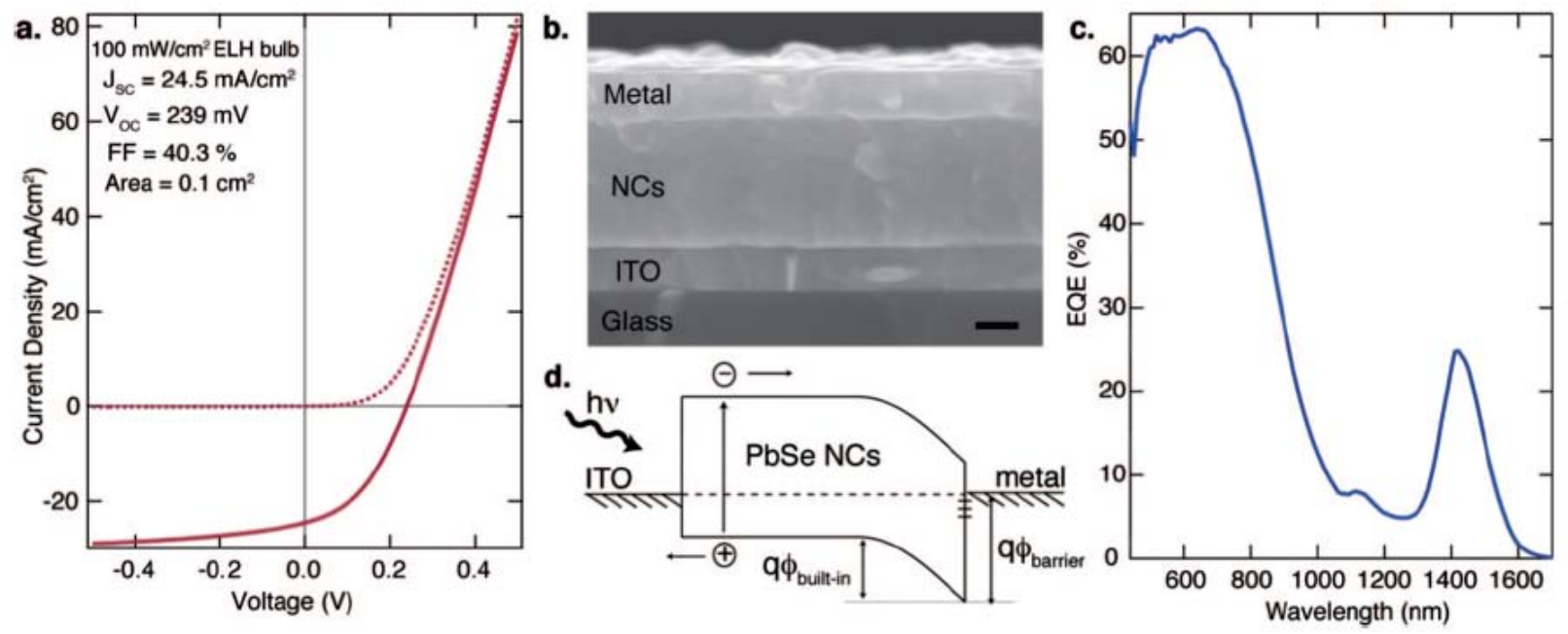

Figure 1.19 Structure, performance and schematics of Schottky solar cell based on colloidal PbSe QD films

(a) I-V characteristics of a representative device. (b) SEM cross-section of the ITO/QD film/metal device. The scale bar represents $100 \mathrm{~nm}$. (c) EQE of a different device with a $140 \mathrm{~nm}$-thick film (Eg=0.95 eV). (d) Proposed equilibrium band diagram. Light is incident through the ITO and band bending occurs at the interface between the QDs and evaporated negative electrode. ${ }^{[37]}$

Sargent's and Nozik's groups recently reported the fabrication and characterization of Schottky photovoltaic devices using $\mathrm{PbS}^{[60]}$ and $\mathrm{PbS}^{[37,61]} \mathrm{QD}$ thin films. The Schottky cells had a simple and essentially similar configuration: QD solids were solution-deposited on ITO-coated glass followed by evaporation of top metal contact (Mg, Al, Ca/Al). The QD films were deposited using either LBL process in which QDs were cross-linked by short-chain dithiol molecules or by spin-casting followed by dipping into a surfactant solution (butylamine, EDT, benzenedithiol, etc.) to exchange original bulky surface ligands with smaller molecules. Figure 1.19 illustrates the basics of Schottky solar cell. Pinning of the Fermi level at the QD-metal interface creates a built-in field that separates photogenerated charge carriers. Electrons are collected at the metal contact, while holes escape into ITO. The performance of the Schottky cell is affected by the built-in potential, the width of the depletion region, and the transport of electron and holes through the QD solid. Most of these parameters can be tailorable by adjusting the morphology, thickness, and chemical treatments of QD thin films. Presently, the best reported power conversion efficiency of PbSe QD-based device is 1.1\% under AM1.5 illumination. ${ }^{[61]}$ 
Devices based on PbS QDs showed efficiencies of 1.8\% under AM1.5 illumination. ${ }^{[60]}$

\subsection{Challenges Facing QD-based Devices}

Despite the recent rapid progress, the development of QD-based devices is still in its infancy. Both the EQE of QD-LEDs and power conversion efficiency of QD solar cells reported in the literature are only a few percent, far below those of state-of-the-art all organic or polymer LEDs and solar cells ${ }^{[62-63]}$.

The room for performance improvement is associated with better understanding of several key material factors with colloidal QDs and QD structures. Due to the large surface/volume ratio of QDs, the presence of surface defects has a pronounced impact on QD properties. In solutions, colloidal QDs are well passivated by insulating and bulky organic ligands introduced during the synthesis. However, in solids, the organic capping layer increases the interparticle spacing and interfacial energy barriers, impeding charge transport and injection

as well as exciton dissociation and energy transfer. ${ }^{[15]}$ Consequently, QD-LEDs suffer from low carrier-injection and energy transfer efficiencies, whereas QD solar cells have low conversion efficiencies due to low charge separation and transport rates. Simple exchange of bulky ligands by smaller organic molecules can significantly improve the electronic coupling, but small molecules are not stable against oxidation and volatility. Introducing a capping nanoshell can greatly improve surface passivation of QDs, but it would also induce a large surface barrier for charge transport and energy transfer.

\subsection{Research Focuses}

It is clear that, in order to develop QD-based optoelectronic devices for efficient energy conversions, it is essential to explore better strategies for surface modification and new device structures which can enable efficient electronic coupling and energy transfer between QDs and 
surrounding materials. This dissertation aims to optimize the synthesis, surface modification, and structure design of CdSe QDs, and achieve high-efficiency energy conversions in QD-based LEDs and solar cell structures. The research has four areas of focuses, which are briefly described below.

Chapter 2 presents the set-up and optimization of colloidal synthesis of CdSe core, core/shell, and core/multishell QDs. The optical properties of the QDs in solutions, including PL and absorption spectra, are systematically studied.

Chapter 3 describes the fabrication process and structure optimization of QD-based LEDs. CdSe/ZnS core/shell QD-LEDs with a hybrid structure of ITO/PEDOT:PSS/poly$\mathrm{TPD} / \mathrm{QDs} / \mathrm{BCP} / \mathrm{Alq}_{3} / \mathrm{LiF} / \mathrm{Al}$ are fabricated and characterized. The thickness of the QD emitting layer is optimized to obtain the best device performance. The EL of the QD-LEDs is further enhanced by introducing an organic layer doped with blue phosphorescent dyes which act as efficient exciton harvesters and energy donors.

In Chapter 4, the exchange of the original organic ligands of colloidal CdSe core and core/shell QDs with inorganic metal chalcogenide ligands $\left(\mathrm{SnS}_{4}{ }^{4-}\right)$ is developed in hydrazine. The process is further optimized in an ammonia hydroxide solution. The optical properties of $\mathrm{SnS}_{4}$-capped QDs in solutions and solids, including PL and absorption spectra, are systematically studied and compared with those of QDs with organic ligands.

In Chapter 5, the electrical and optical properties of $\mathrm{SnS}_{4}$-capped QD thin films are characterized, and their applicability for energy conversions is investigated. QD-LEDs based on $\mathrm{SnS}_{4}$-capped CdSe based core/multishell QDs are fabricated via a layer-by-layer method. Furthermore, $\mathrm{SnS}_{4}$-capped CdSe core QDs for photovoltaic conversion is evaluated by studying the photocurrent response of a simple ITO/QDs/Al structure. 


\section{Chapter 2 Synthesis and Characterization of Colloidal}

\section{Semiconductor Quantum Dots}

Colloidal II-VI semiconductor QDs have attracted much attention because of their unique electronic and optical properties and potential for high-efficiency energy conversion devices. For example, CdSe QDs can be engineered as efficient emitters for developing QD-LEDs with high color purity (narrow emission band) and tunable emission wavelength over visible range. ${ }^{[15]}$

The exploration for QD device applications is based on the recent rapid progress in the synthesis of high-quality colloidal QDs. As discussed in section 1.1.3, low-cost and controllable synthetic protocols have been developed for syntheses of core QDs as well as core/shell QDs. In this chapter, we develop in-house capability of CdSe core QDs synthesis using a modified hotinjection method published by Murray ${ }^{[2]}$ and Peng ${ }^{[32]}$. In order to demonstrate the versatility of this method, CdS and CdTe core QDs are synthesized via the same method as well. In addition, CdSe/ZnS core/shell QDs are synthesized via the SILAR method developed by Peng ${ }^{[28]}$. Finally, in order to obtain higher PL QYs and better stability, CdSe/CdS/Cd $\mathrm{Cd}_{0.5} \mathrm{Zn}_{0.5} \mathrm{~S} / \mathrm{ZnS}$ core/multishell QDs with reduced interfacial strain are designed and synthesized.

\subsection{CdS, CdSe and CdTe Core QDs}

\subsubsection{CdSe Core QDs}

A modified hot-injection method was used to synthesize CdSe core QDs. Typically, the mixture of $0.2 \mathrm{mmol} \mathrm{CdO}, 0.254 \mathrm{~mL}$ oleic acid (OA) and $10.152 \mathrm{~mL}$ 1-octadecene (ODE) in a three-necked flask was heated to about $280{ }^{\circ} \mathrm{C}$ under $\mathrm{N}_{2}$ atmosphere to obtain a clear solution. After the solution was cooled to room temperature, octadecylamine (ODA) (3.6 g) and 
trioctylphosphine oxide (TOPO) $(1.2 \mathrm{~g})$ were added and the solution was reheated to $280{ }^{\circ} \mathrm{C}$. At this temperature, a selenium solution made by dissolving $2.5 \mathrm{mmol}$ selenium in trioctylphosphine (TOP) (1.766 mL) and ODE (3 mL) was quickly injected. After the injection, the temperature was maintained at $260{ }^{\circ} \mathrm{C}$ for the growth of CdSe QDs. After the growth step, the heating mantle was removed to cool the solution to room temperature. An extraction procedure was then used to purify the QDs by removing the side products and unreacted precursors ${ }^{[32]}$. Figure 2.1 illustrates the process of hot-injection method.

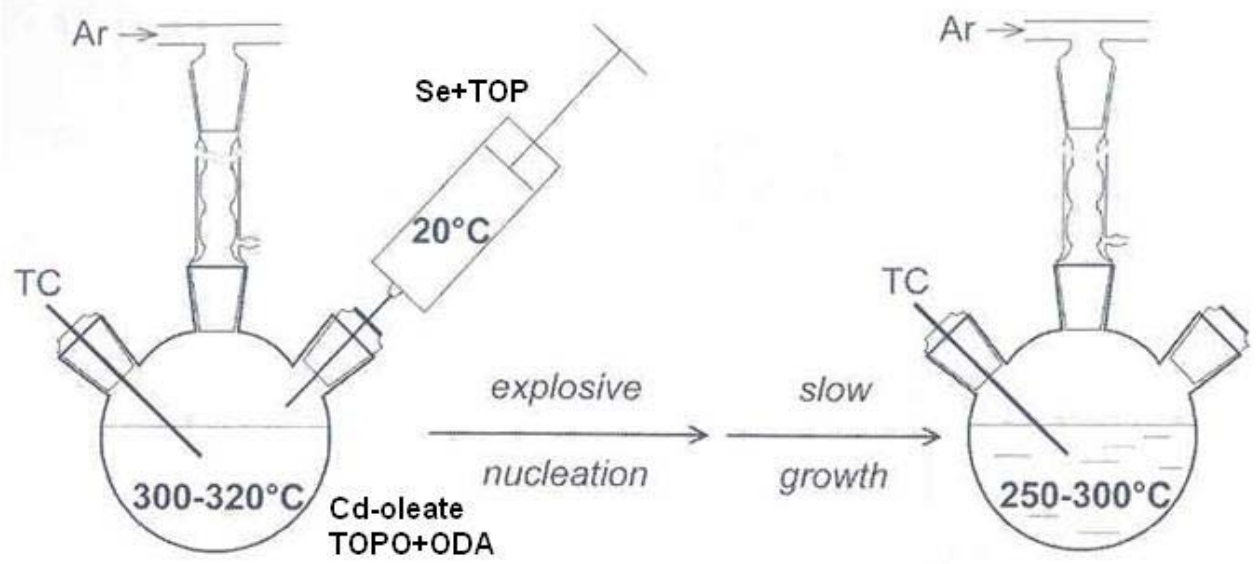

Figure 2.1 Cartoon depicting the hot-injection method for synthesis of CdSe core QDs.

The absorption and photoluminescence (PL) spectra of CdSe QDs in hexane solvent were measured using a Hitachi U-3900H UV-Vis spectrophotometer and a Hitachi F-7000 fluorescence spectrophotometer (365 nm excitation), respectively.

Figure 2.2 displays the temporal evolution of the absorption and PL spectra of CdSe QDs grown at $260{ }^{\circ} \mathrm{C}$ for different amounts of time through the reaction of CdO-OA in ODA-TOPOODE with the injected selenium dissolved in TOP-ODE. As the growth time increases, the first excitonic peak of the obtained CdSe QDs gradually shifts to the longer wavelength from $481 \mathrm{~nm}$ to $548 \mathrm{~nm}$ due to the increased particle size, and the PL peak gradually shifts to the longer wavelength from $495 \mathrm{~nm}$ to $557 \mathrm{~nm}$. The largest QDs resulted from 420 sec growth retain the sharp excitonic absorption and PL peaks. The narrow full width at half maximum (FWHM) of 
the band edge PL spectra (27-30 nm of FWHM) of all the QDs suggests that the resulting QDs in each solution are nearly monodispersed and have narrow size distribution.
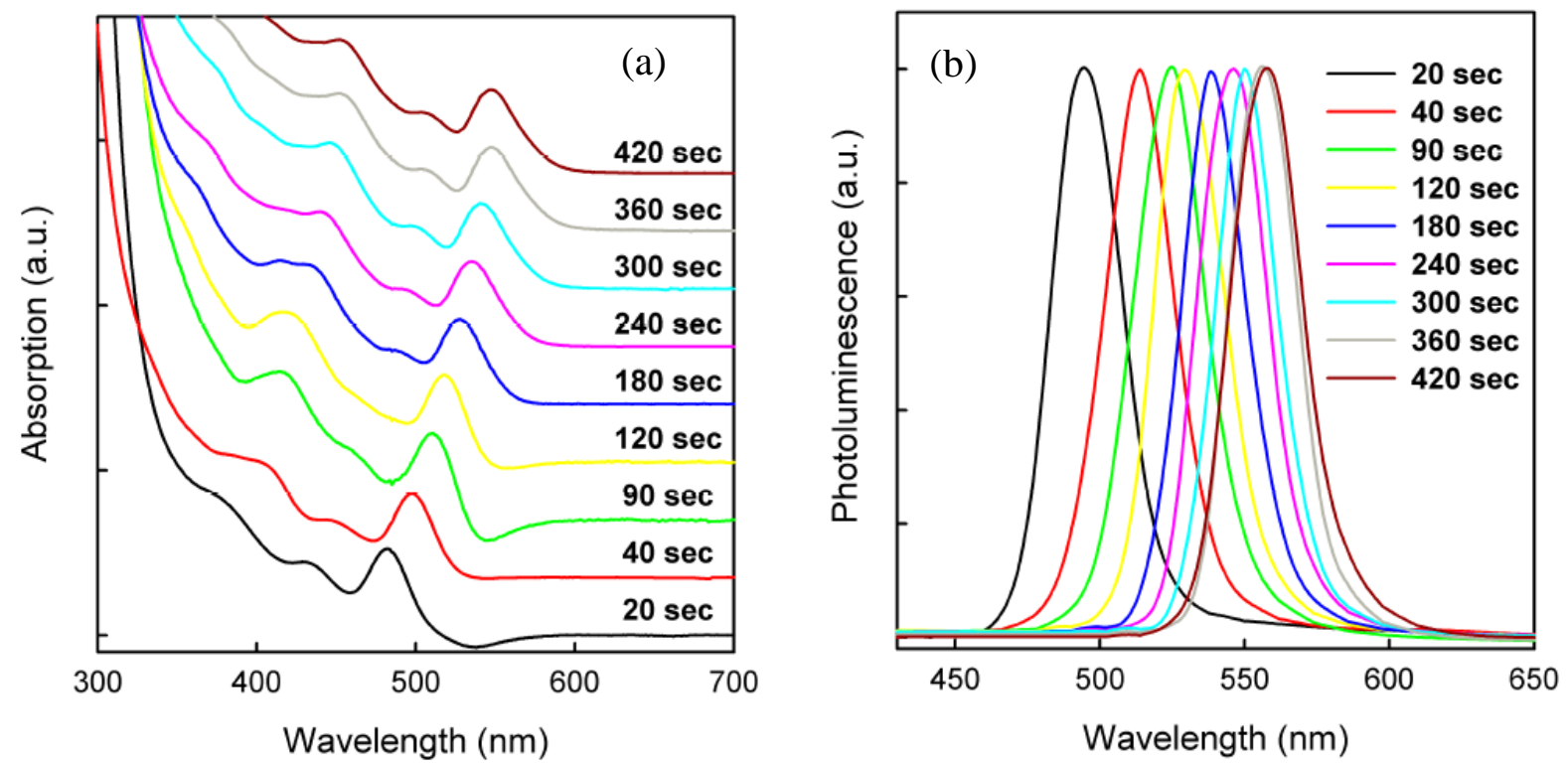

Figure 2.2 Temporal evolution of (a) absorption and (b) PL spectra of CdSe QDs samples taken during the growth reaction at $260{ }^{\circ} \mathrm{C}$

The sizes of the QDs can be calculated based on their first excitonic absorption peak positions using the following empirical equation, derived by Peng and co-workers ${ }^{[32]}$ :

$$
\mathrm{D}=\left(1.6122 \times 10^{-9}\right) \lambda^{4}-\left(2.6575 \times 10^{-6}\right) \lambda^{3}+\left(1.6242 \times 10^{-3}\right) \lambda^{2}-(0.4277) \lambda+(41.57)
$$

Where D $(\mathrm{nm})$ is the diameter of a given QD sample, and $\lambda(\mathrm{nm})$ is the wavelength of the first excitonic absorption peak of the sample. The evolution of the particle size during growth of the CdSe QDs at $260{ }^{\circ} \mathrm{C}$ was calculated and is shown in Figure 2.3. As seen, the growth slows down as the process proceeds. After $420 \mathrm{sec}$, the particle diameter reaches $\sim 3.0 \mathrm{~nm}$. 


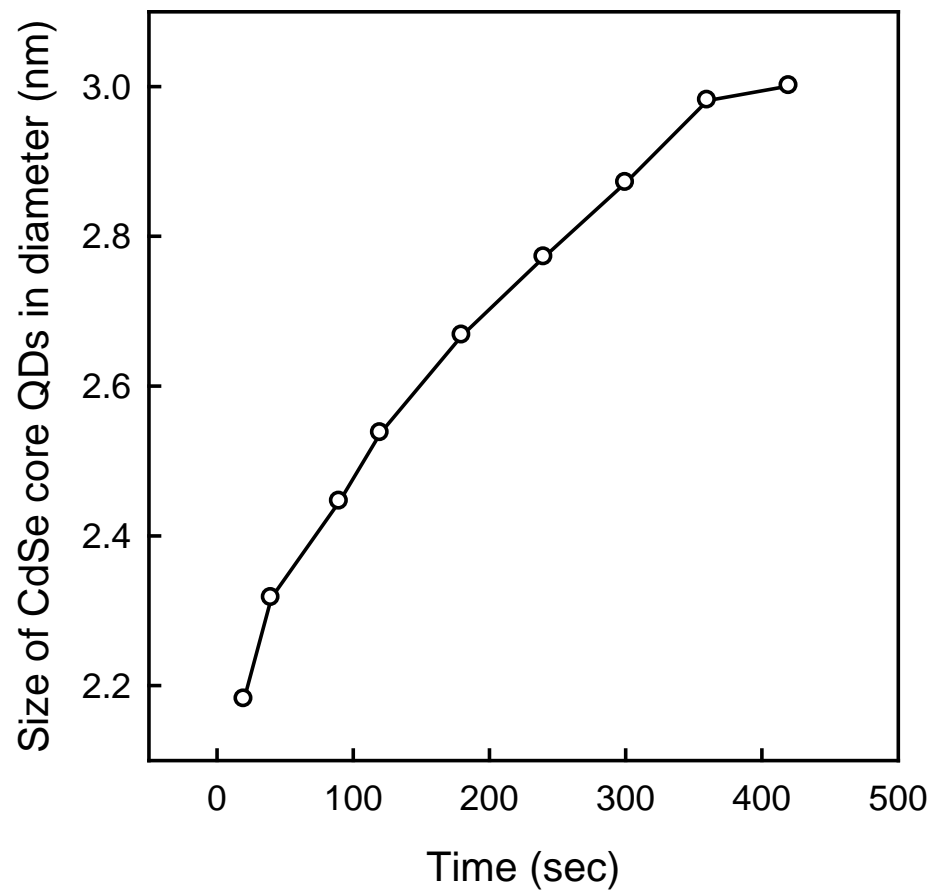

Figure 2.3 Temporal dependence of the particle size during the growth of CdSe QDs at $260{ }^{\circ} \mathrm{C}$

The absorption spectrum as well as the PL spectrum of CdSe QDs of 2.5 nm in diameter is shown in Figure 2.4. The first excitonic absorption peak occurs at $515 \mathrm{~nm}$, whereas the PL emission is centered at $530 \mathrm{~nm}$. The spectrum peak shift between absorption and PL spectra is known as the Stokes shift. The observed $15 \mathrm{~nm}$ Stokes shift is consistent with the data reported in the literature ${ }^{[32]}$. The existence of multiple excitonic absorption peaks and the narrow FWHM of $28 \mathrm{~nm}$ corroborates that the as-prepared CdSe core QDs are uniform in size and shape. Based on absorption and PL measurements, the PL QY of the CdSe core QDs in hexane was determined to be $\sim 10 \%$, suggesting good passivation of the core QDs by the ODA-TOPO ligands. 


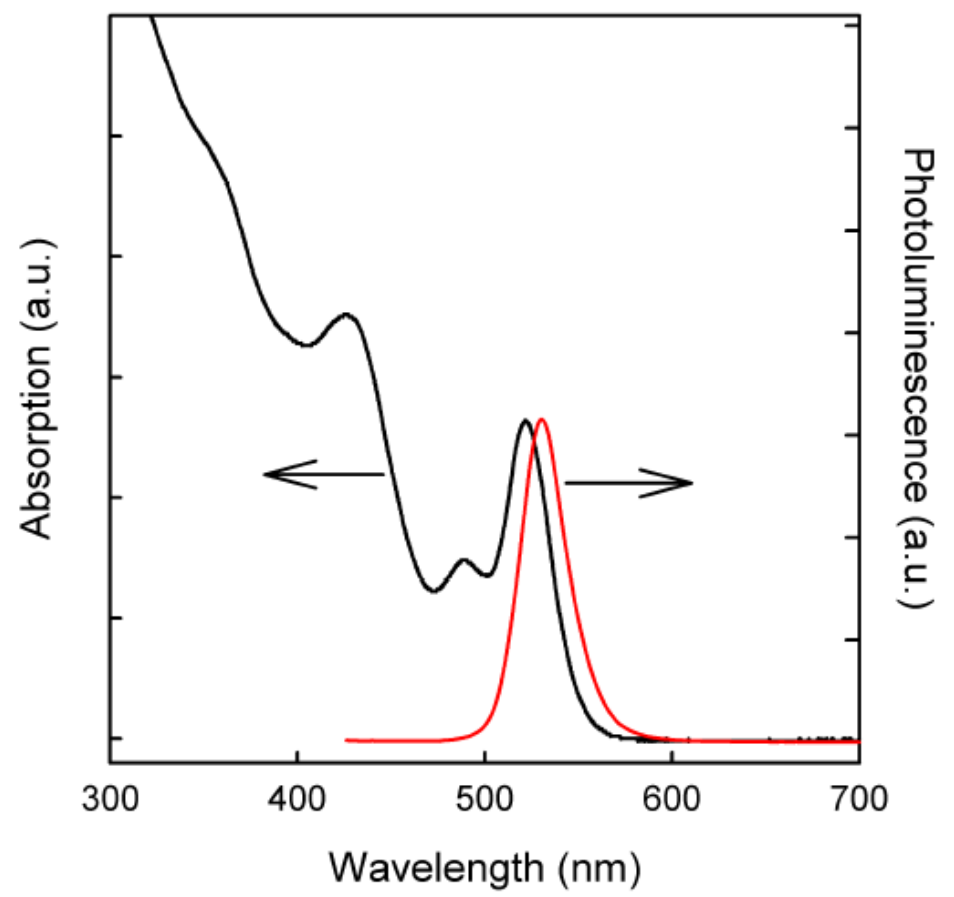

Figure 2.4 Absorption and PL spectrum of CdSe QDs emitting green light (530 nm)

\subsubsection{CdS Core QDs}

A modified hot-injection method was used to synthesize blue and UV CdS core QDs. Typically, the mixture of $1 \mathrm{mmol} \mathrm{CdO}, 1.27 \mathrm{~mL}$ OA and $15 \mathrm{~mL}$ ODE in a three-necked flask was heated to about $280{ }^{\circ} \mathrm{C}$ under $\mathrm{N}_{2}$ atmosphere to obtain a clear solution. After the solution was cooled to room temperature, ODA (3.6 g) and TOPO (1.2 g) were added and the solution was reheated to $280^{\circ} \mathrm{C}$. A sulfur solution was prepared by dissolving $1 \mathrm{mmol}$ sulfur in ODE ( $5 \mathrm{~mL}$ ) at $180{ }^{\circ} \mathrm{C}$ for $30 \mathrm{~min}$, and then cooled down to room temperature. At $280^{\circ} \mathrm{C}$, the sulfur solution was quickly injected. After the injection, the temperature was adjusted to $260{ }^{\circ} \mathrm{C}$ for ideal time to allow the growth of CdS QDs. After this time, the heating mantle was removed to cool the solution to room temperature, and an extraction procedure was used to purify the QDs from side products and unreacted precursors ${ }^{[32]}$.

Similar as the optical characterization of CdSe core QDs, Figure 2.5 displays the 
temporal evolution of the absorption spectra of the CdS QDs, which were produced at $260{ }^{\circ} \mathrm{C}$ through the reaction of CdO-OA in ODA-TOPO-ODE with the injected sulfur dissolved in ODE. During a 900 sec growth progress at the reaction temperature, the first excitonic peaks of the obtained CdSe QDs gradually shift to longer wavelength from $371 \mathrm{~nm}$ to $414 \mathrm{~nm}$ due to the increase of particle size. After 900 sec growth, the original sharp excitonic absorption peak is still retained. The sharp feature of the multiple excitonic absorption peaks demonstrates that the size of the resulting CdS QDs is nearly monodispersed with narrow size distribution.

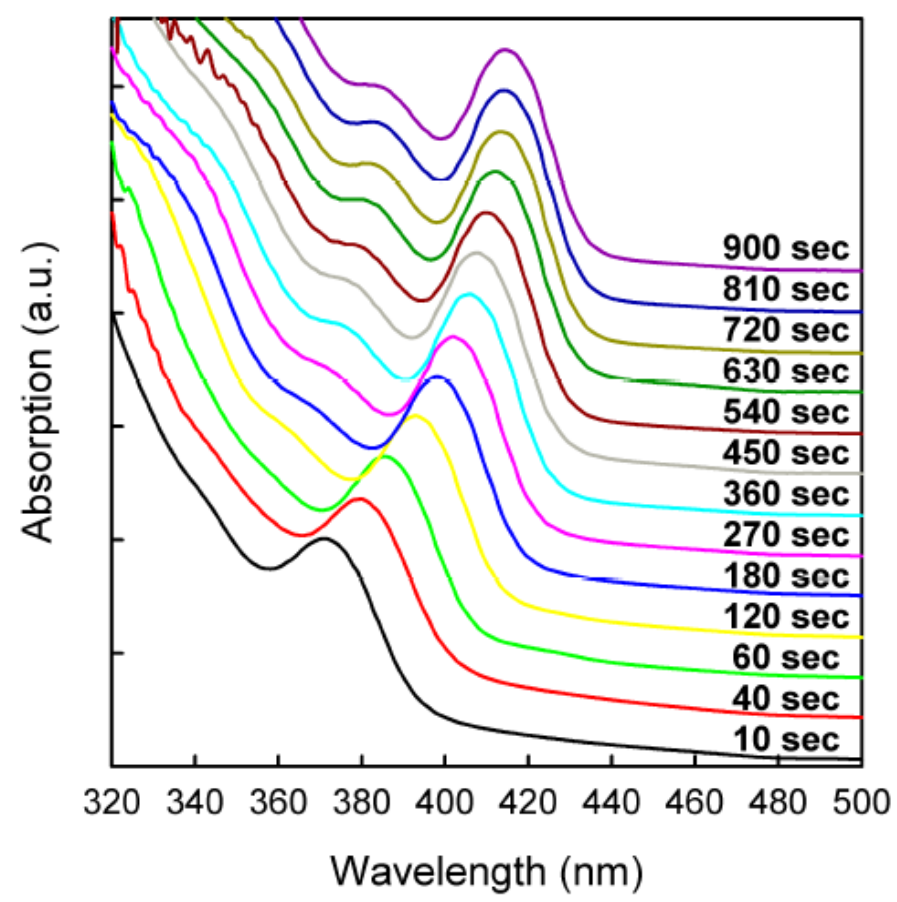

Figure 2.5 Temporal evolution of absorption spectra of CdS QDs samples taken during the growth reaction at $260{ }^{\circ} \mathrm{C}$

Empirical function correlating the size of CdS QDs with the position of the first excitonic peak in its absorption spectra is listed below ${ }^{[32]}$ :

$$
\mathrm{D}=\left(-6.6521 \times 10^{-8}\right) \lambda^{3}+\left(1.9557 \times 10^{-4}\right) \lambda^{2}-\left(9.2352 \times 10^{-2}\right) \lambda+(13.29)
$$

In the above function, $\mathrm{D}(\mathrm{nm})$ is the diameter of a given QD sample, and $\lambda(\mathrm{nm})$ is the wavelength of the first excitonic absorption peak of the corresponding sample. Based on this function, the evolution of the particle size during growth of the CdS QDs at $260{ }^{\circ} \mathrm{C}$ can be 
calculated as shown in Figure 2.6. The growth rate decreased gradually and the growth almost terminated when the particles diameter reached $\sim 3.8 \mathrm{~nm}$.

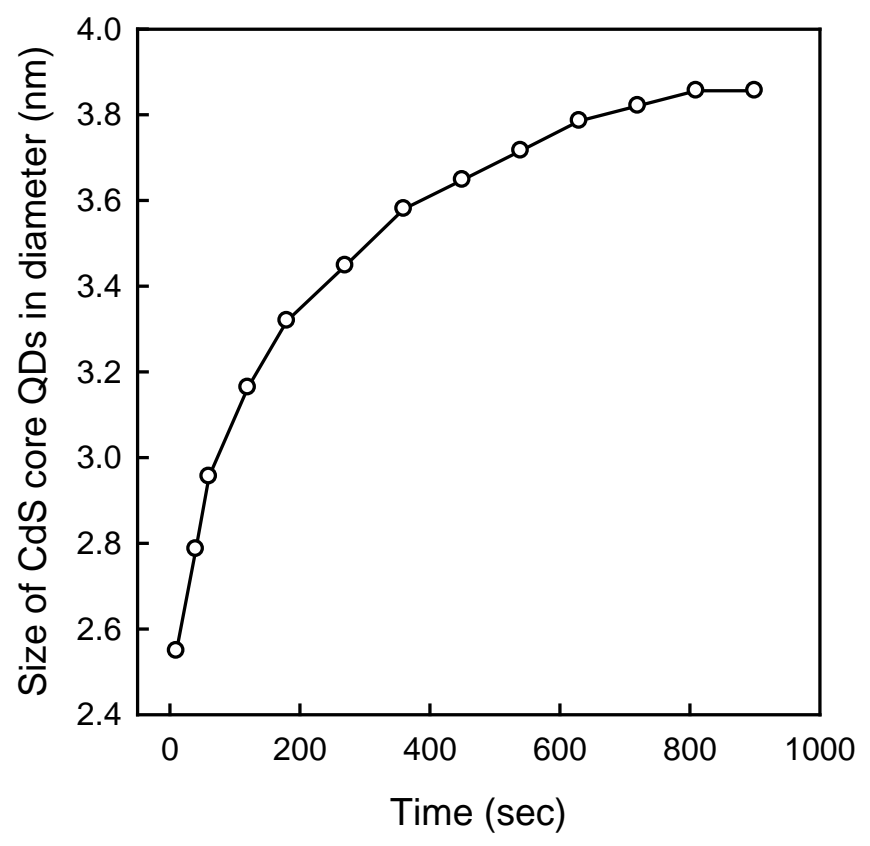

Figure 2.6 Temporal dependence of the particle size during the growth of CdS QDs at $260{ }^{\circ} \mathrm{C}$

The absorption spectrum as well as the PL spectrum of CdS QDs with $\sim 4.2 \mathrm{~nm}$ in diameter is shown in Figure 2.7. The first excitonic absorption peak occurs at $423 \mathrm{~nm}$ with a corresponding PL emission at $435 \mathrm{~nm}$. The Stokes shift of $12 \mathrm{~nm}$ is consistent with the data from the literature ${ }^{[32]}$. In addition, the existence of the discrete multiple excitonic absorption peak and the narrow FWHM of $20 \mathrm{~nm}$ corroborates that the as-prepared CdS core QDs have a very narrow size distribution and uniform size. 


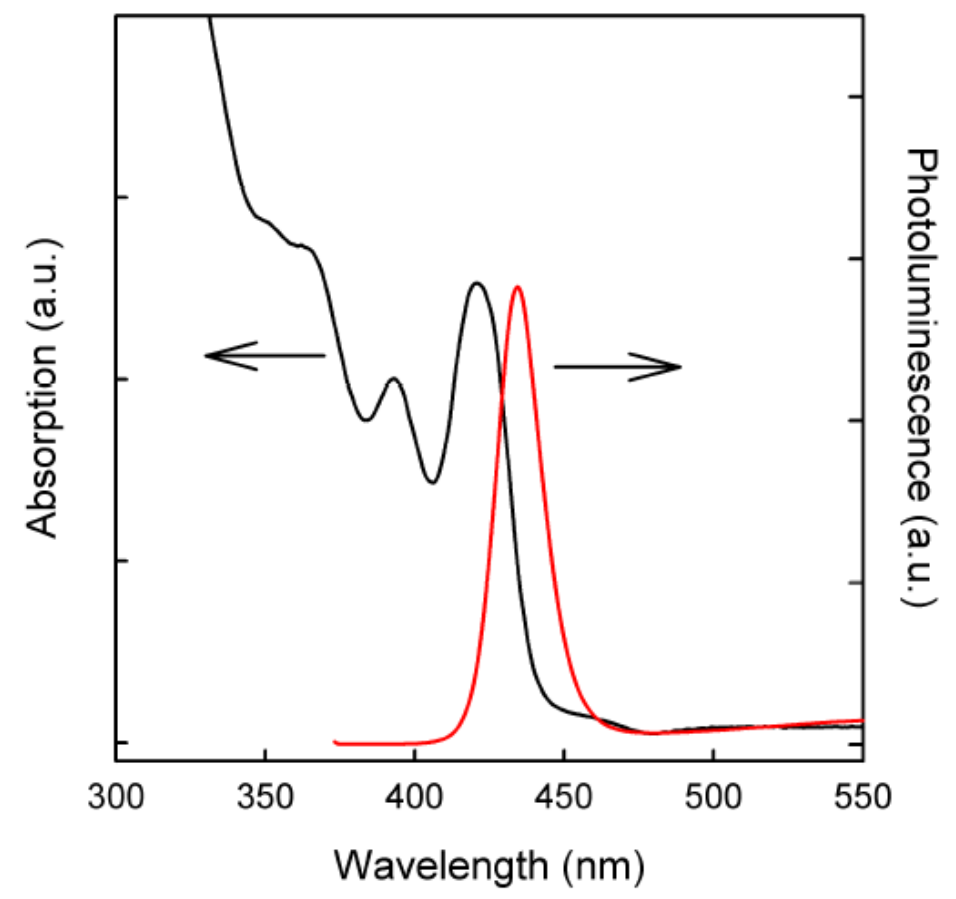

Figure 2.7 Absorption and PL spectrum of CdS QDs emitting deep blue light (435 nm)

\subsubsection{CdTe Core QDs}

A modified hot-injection method was used to synthesize red CdTe core QDs. Typically, the mixture of $4 \mathrm{mmol} \mathrm{CdO}, 5.08 \mathrm{~mL} \mathrm{OA}$ and $30 \mathrm{~mL}$ ODE in a three-necked flask was heated to about $280{ }^{\circ} \mathrm{C}$ under $\mathrm{N}_{2}$ atmosphere to obtain a clear solution. After the solution was cooled to room temperature, ODA (7.5 g) and TOPO (2.5 g) were added and the solution was reheated to $280^{\circ} \mathrm{C}$. At this temperature, a tellurium solution made by dissolving $2 \mathrm{mmol}$ tellurium in TOP $(5.5 \mathrm{~mL})$ and ODE $(6 \mathrm{~mL})$ was quickly injected. After the injection, the temperature was adjusted to $250^{\circ} \mathrm{C}$ for ideal time to allow the growth of CdTe QDs. After this time, the heating mantle was removed to cool the solution to room temperature, and an extraction procedure was used to purify the QDs from side products and unreacted precursors ${ }^{[32]}$.

Figure 2.8 displays the temporal evolution of the absorption and PL spectra of the CdTe QDs, which were produced at $250{ }^{\circ} \mathrm{C}$ through the reaction of CdO-OA in ODA-TOPO-ODE with the injected tellurium dissolved in TOP-ODE. During a $3600 \mathrm{sec}$ growth progress at the 
reaction temperature, the first excitonic peaks of the obtained CdTe QDs gradually shift to longer wavelength from $648 \mathrm{~nm}$ to $688 \mathrm{~nm}$ due to the increase of particle size. The PL peaks gradually shift to longer wavelength from $656 \mathrm{~nm}$ of $30 \mathrm{sec}$ growth to $673 \mathrm{~nm}$ of $600 \mathrm{sec}$ growth, while there is no evident PL spectra obtained for the QD smaples grown for 1800 sec and 3600 sec. After 3600 sec growth, the excitonic absorption peak is not as evident as the initial one (for instance, absorption spectrum grown for $30 \mathrm{sec}$ ), indicating that Ostwald ripening may occur. Although the multiple excitonic absorption peaks are still identified, these peaks are less discrete than the previous CdSe and CdS ones (Figure 2.2 and Figure 2.5). In addition, the FWHM of PL spectra for CdTe QDs exhibits a relatively large range of 37-42 $\mathrm{nm}$. This is mainly originated from the week control of the surface ligands of ODA and TOPO induced during the CdTe QDs synthesis. Replacing ODA and TOPO with n-tetradecylphosphonice acid (TDPA) may improve the quality of CdTe QDs. ${ }^{[32]}$
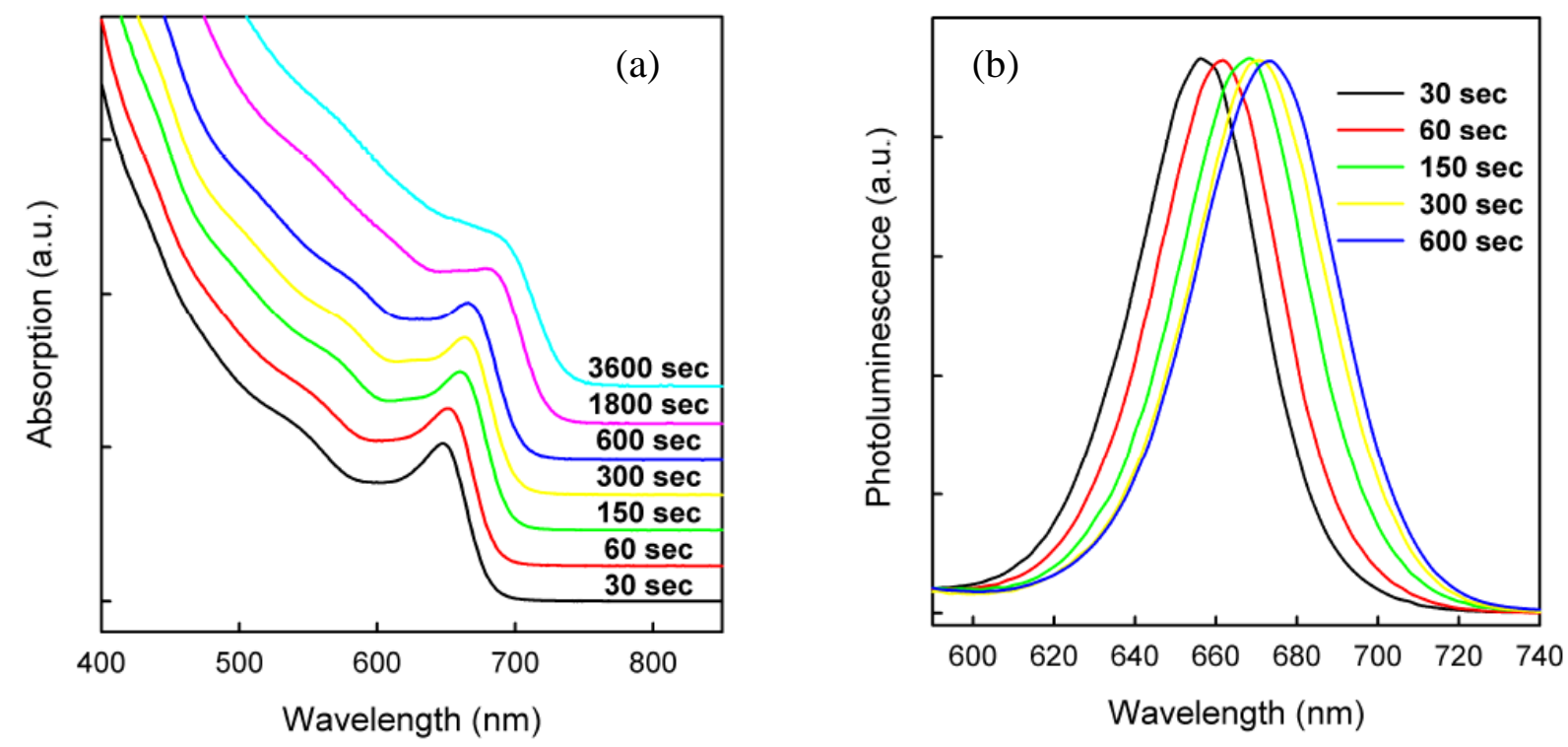

Figure 2.8 Temporal evolution of (a) absorption and (b) PL spectra of CdTe QDs samples taken during the growth reaction at $250^{\circ} \mathrm{C}$. There is no evident PL spectra tested for the QDs samples grown for 1800 sec or 3600 sec.

Empirical function correlating the size of CdTe QDs with the position of the first excitonic peak in its absorption spectra is listed below ${ }^{[32]}$ : 


$$
\mathrm{D}=\left(9.8127 \times 10^{-7}\right) \lambda^{3}-\left(1.7147 \times 10^{-3}\right) \lambda^{2}+(1.0064) \lambda-(194.84)
$$

In the above function, $\mathrm{D}(\mathrm{nm})$ is the diameter of a given QD sample, and $\lambda(\mathrm{nm})$ is the wavelength of the first excitonic absorption peak of the corresponding sample. Based on this function, the evolution of the particle size during growth of the CdTe QDs at $250{ }^{\circ} \mathrm{C}$ can be calculated as shown in Figure 2.9. CdTe QDs were gradually grown from $4.3 \mathrm{~nm}$ in diameter to $5.5 \mathrm{~nm}$ in diameter.

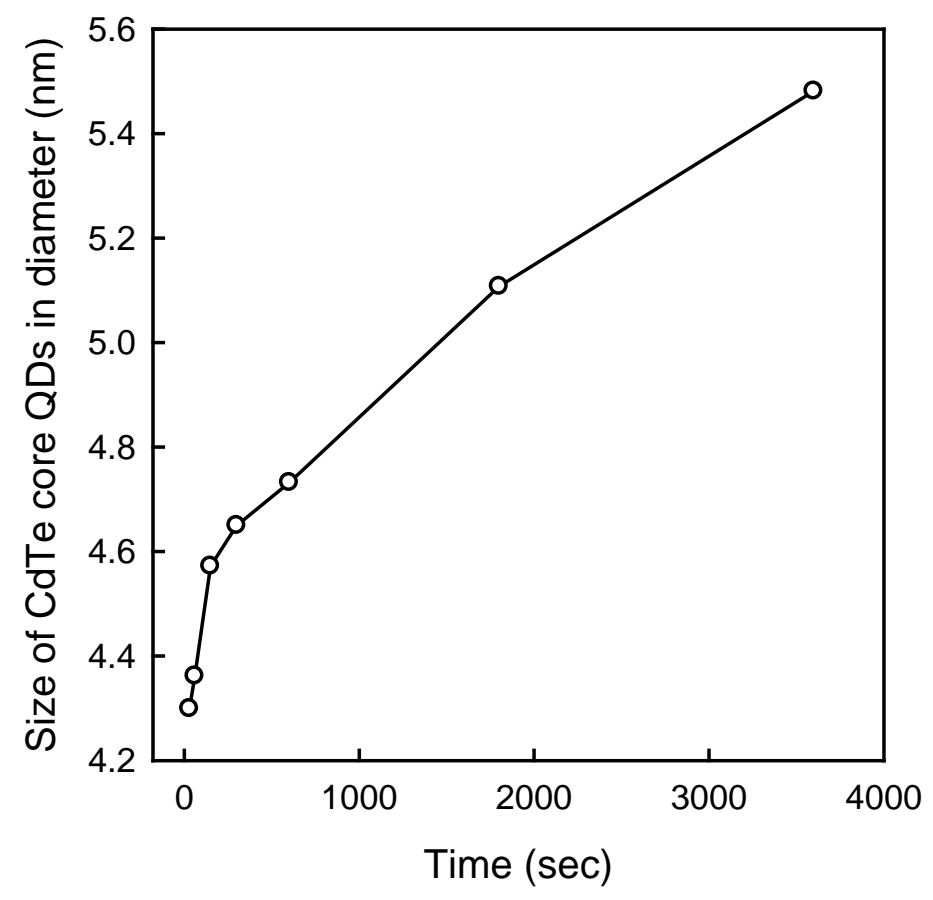

Figure 2.9 Temporal dependence of the particle size during the growth of CdTe QDs at $250{ }^{\circ} \mathrm{C}$

The absorption spectrum as well as the PL spectrum of CdTe QDs with $\sim 4.6 \mathrm{~nm}$ in diameter is shown in Figure 2.10. The first excitonic absorption peak occurs at $665 \mathrm{~nm}$ with a corresponding PL emission at $672 \mathrm{~nm}$. The CdTe core QDs show a relatively weak PL band redshifted from the absorption edge. This may result from the radiative recombination of electrons and holes trapped in surface defect states. The FWHM of PL spectrum is around $37 \mathrm{~nm}$. 


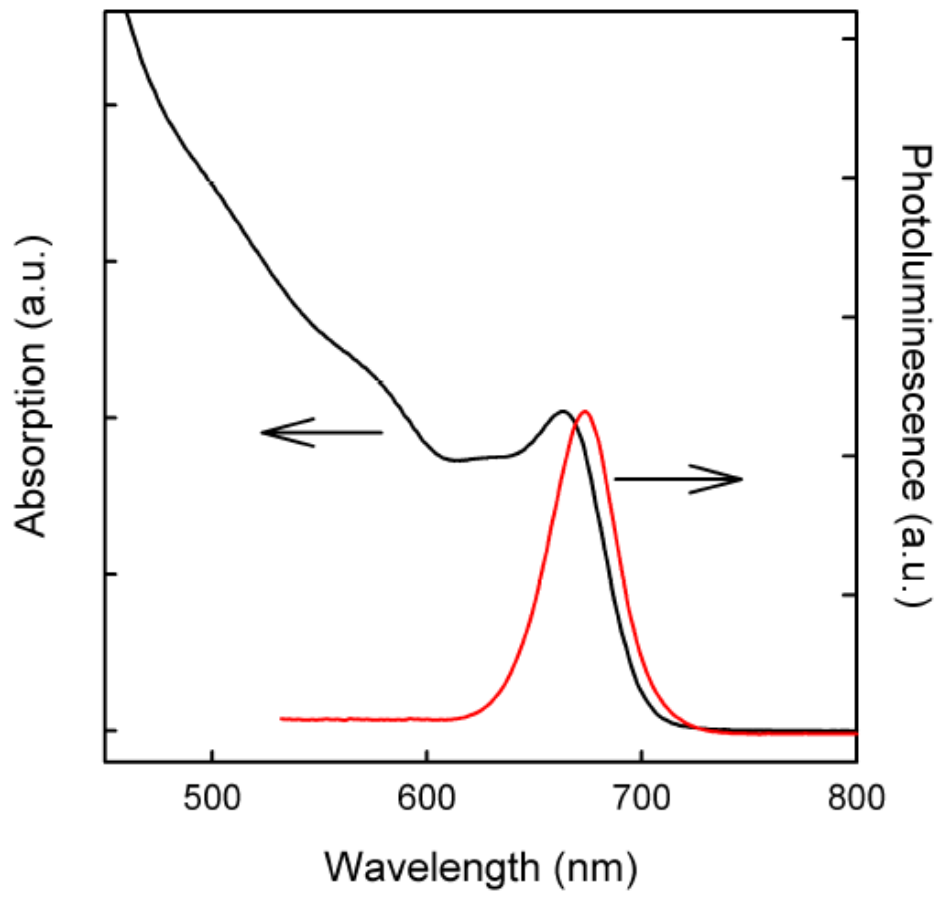

Figure 2.10 Absorption and PL spectrum of CdTe QDs emitting deep red light (670 nm)

\subsection{CdSe/ZnS Core/Shell QDs}

Core/shell QDs are better emitters than the core QDs in QD-LEDs, due to better passivation of the core QDs by a nanoshell, leading to enhanced luminescence QYs and greater stability against the harsh processing conditions. ${ }^{[15]}$ This task aims to grow high-quality CdSe/ZnS core/shell QDs by the SILAR method. ${ }^{[28]}$ In the process, the ZnS shell is grown one monolayer at a time by alternating injections of air-stable and inexpensive cationic ( $\mathrm{Zn}-$ ) and anionic(S-) precursors into the reaction mixture with CdSe core QDs, as illustrated in Figure 2.11. 


\section{SILAR method}

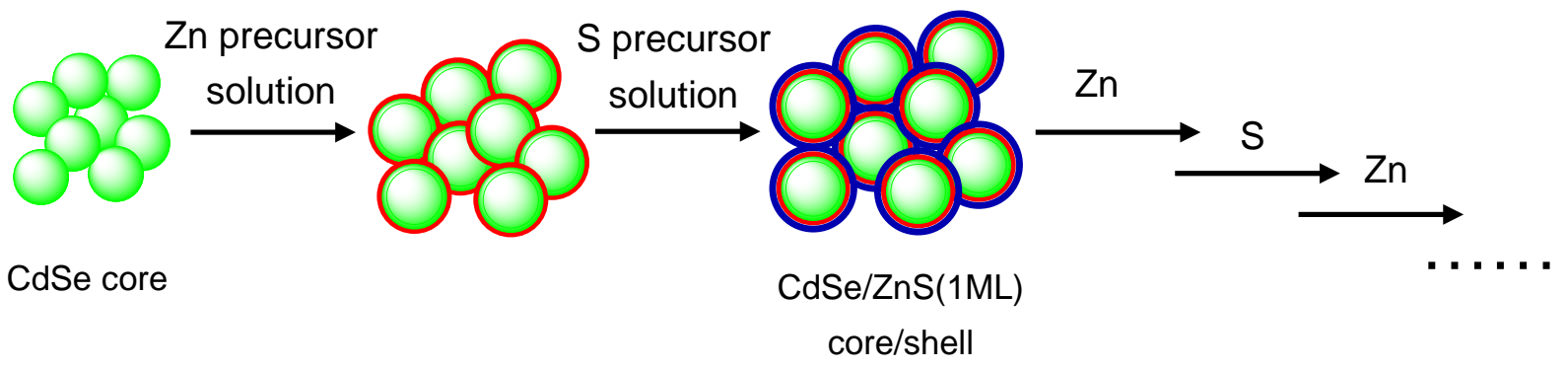

Figure 2.11 Illustration of CdSe/ZnS core/shell QDs synthesis using the SILAR method

As shown in Figure 1.7, the bandgap of the $\mathrm{ZnS}$ shell material $(3.7 \mathrm{eV})$ is larger than that of the CdSe core $\left(1.96 \mathrm{eV}^{2.64} \mathrm{eV}^{[64]}\right)$. Also, the conduction band of the $\mathrm{ZnS}$ shell is of higher energy than that of the CdSe core, while the valence band of the $\mathrm{ZnS}$ shell is of lower energy than that of the CdSe core. This core/shell structure thus has type-I band alignment ${ }^{[26]}$ and enables effective confinement of electrons and holes in the core material. Consequently, a significant improvement in QDs emission QYs can be achieved. ${ }^{[26]}$

In a typical synthesis of CdSe/ZnS core/shell QDs, CdSe core QDs (2.68 nm in diameter, $7.35 \times 10^{-7}$ mol of particles) were prepared using the recipe described earlier and mixed with 18 $\mathrm{mL}$ of ODE and $6 \mathrm{~g}$ of ODA in a three-necked flask. The mixture was heated to $235{ }^{\circ} \mathrm{C}$ in $\mathrm{N}_{2}$ atmosphere, and Zn precursor and S precursor were injected alternatively into the flask for the shell layer growth in the order of Zn-S with an interval of 10 min between two successive injections (see details in Appendix 1). The reaction mixture was allowed to cool to room temperature. This was followed by QD extraction procedure ${ }^{[32]}$. Further purification of CdSe/ZnS core/shell QDs was done by precipitating them with acetone or methanol. Highly pure QDs were obtained for device fabrication by repeating above purification procedure for 2-3 times. Aliquots of the solution were removed after each monolayer of shell growth with a syringe and tested for optical spectra. 


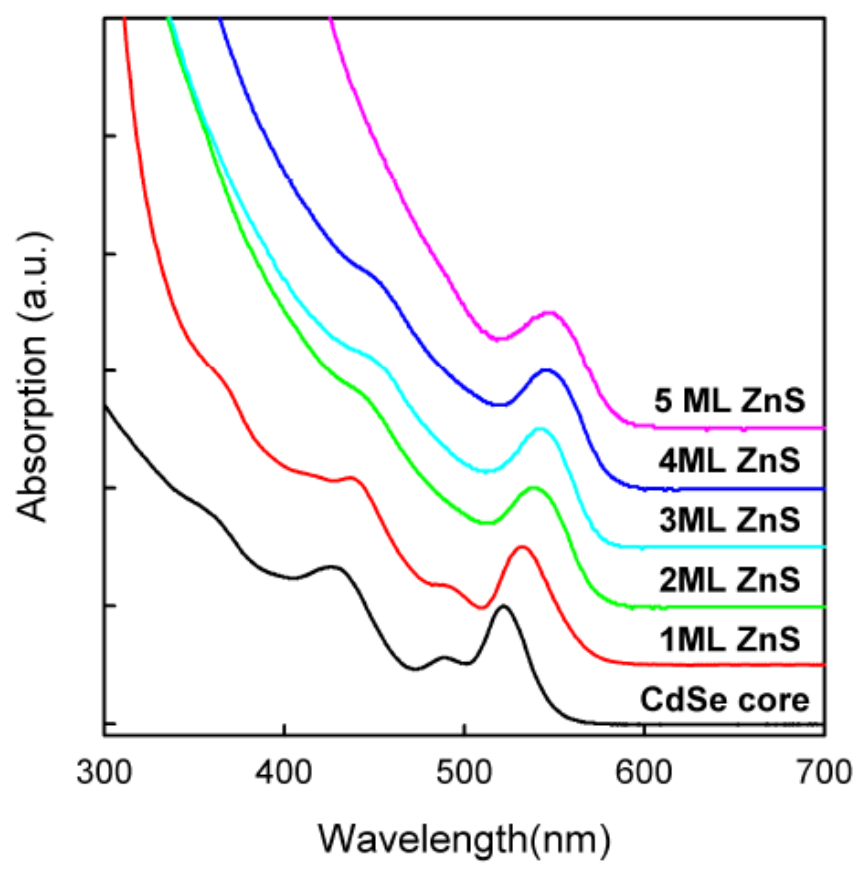

Figure 2.12 Absorption spectra of CdSe core and CdSe/ZnS core/shell QDs with different numbers of ZnS monolayers (MLs)

Figure 2.12 displays the evolution of the absorption spectra upon consecutive growth of the ZnS shell. It can be seen that, during the shell growth, the first excitonic absorption peak gradually shifts to the longer wavelength from $530 \mathrm{~nm}$ to $550 \mathrm{~nm}$. This phenomenon can be explained based on the band structure of the CdSe/ZnS core/shell QDs: the wave function of light electrons tunnels into the ZnS shell, while the wave function of heavy holes has a negligible probability of spreading into the ZnS shell. The increased delocalization of the electrons, in the $\mathrm{CdSe} / \mathrm{ZnS}$ core/shell structure, lowers its confinement energy and therefore the energy of the excited state, leading to a red shift in the optical spectra when the ZnS shell is grown on the CdSe core QDs. 


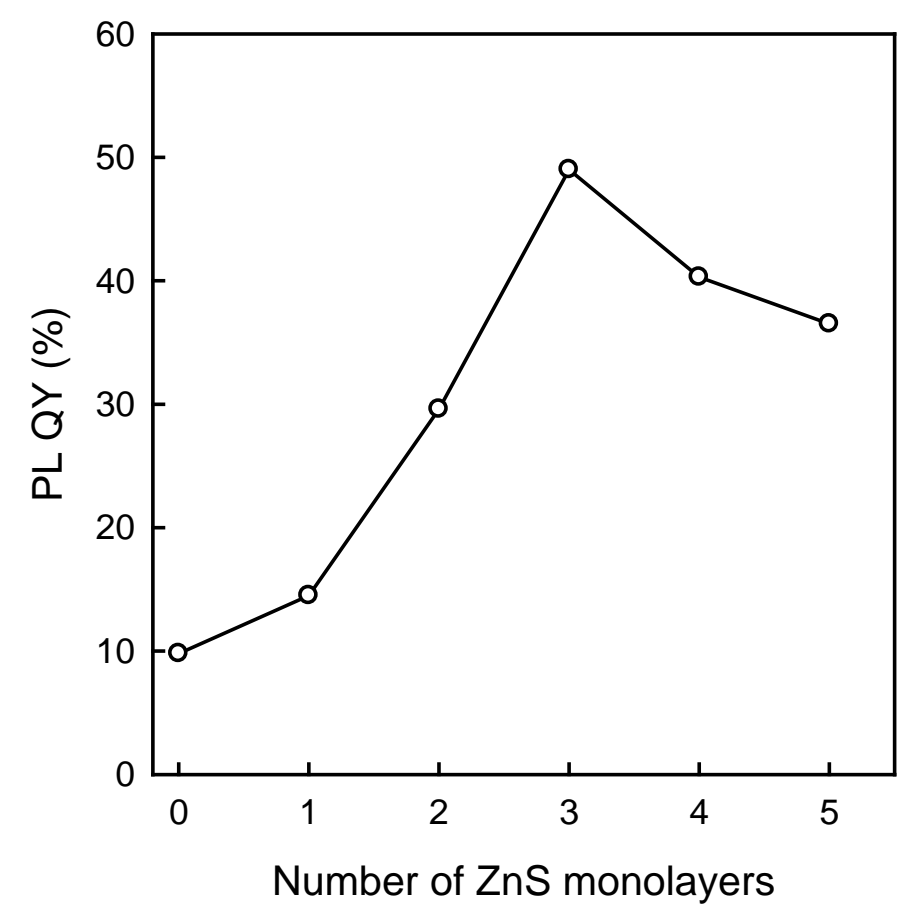

Figure 2.13 Evolution of the photoluminescence quantum yield of CdSe/ZnS core/shell QDs

The evolution of the PL QY of CdSe/ZnS core/shell QDs is shown in Figure 2.13. The QY of the CdSe core QDs is on the order of 10\%. After coating with 3 MLs of ZnS shell, the QY is increased from $\sim 10 \%$ to $\sim 50 \%$. Additional growth of $\mathrm{ZnS}$ leads to an evident decrease in the QY. The drop in the QY is due to the formation of defects at the core/shell interface. ${ }^{[65]}$ Because a large lattice mismatch of $12 \%$ exists between CdSe and ZnS, lattice strain increases as the ZnS shell grows thicker. ${ }^{[65]}$ A partial relaxation of the strain may lead to misfit defect formation. Figure 2.14 shows the typical absorption and PL spectra of CdSe QDs before and after the growth of a 3-monolayer ZnS shell. These green emitting CdSe/ZnS core/shell QDs will be used to fabricate QD-LEDs in Chapter 3. 


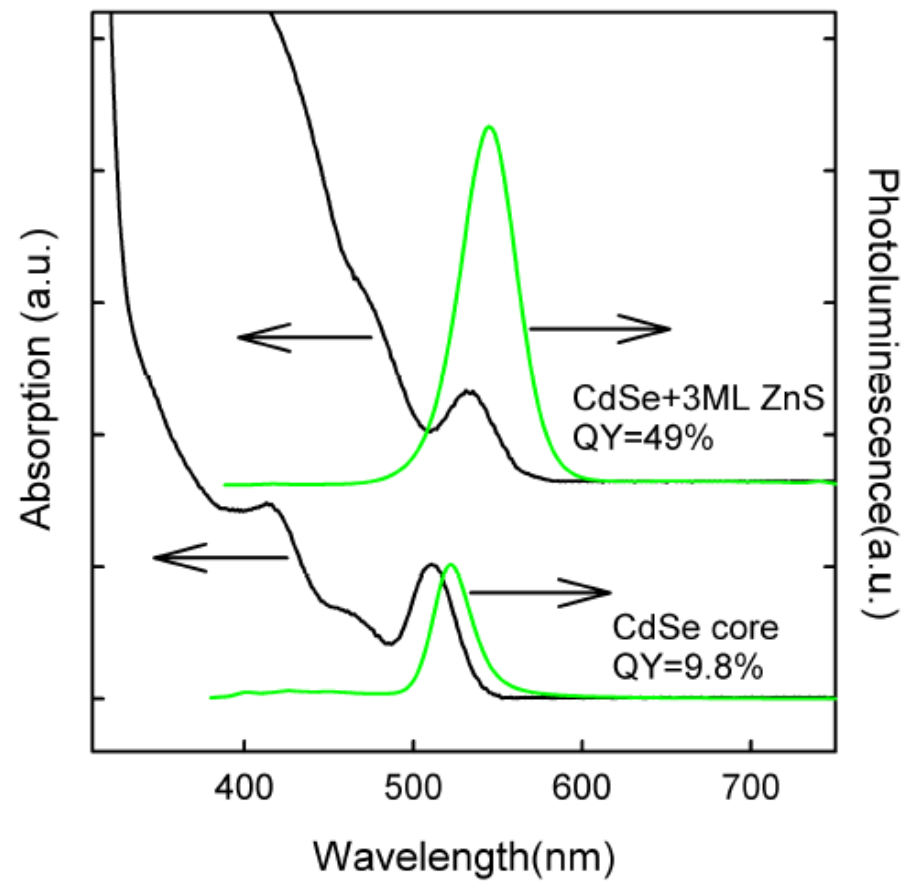

Figure 2.14 PL spectra and absorption spectra of green-emitting CdSe core QDs and CdSe/ZnS QDs

\subsection{CdSe/CdS/Zn ${ }_{0.5} \mathrm{Cd}_{0.5} \mathrm{~S} / \mathrm{ZnS}$ Core/Multishell QDs}

As discussed in section 2.2, the ZnS shell should provide ideal passivation of CdSe core QDs, but the large lattice mismatch (12\%) makes it difficult to grow CdSe/ZnS core/shell QDs with the PL QD approaching 100\%. CdS has a smaller lattice mismatch(3.9\%) with CdSe. ${ }^{[43]}$ Therefore, doping the $\mathrm{ZnS}$ shell with Cd may effectively relieve some of the strain resulted from the large lattice mismatch (ZnS vs. CdSe) and a thicker shell can be grown. A $\mathrm{CdSe} / \mathrm{CdS} / \mathrm{Zn}_{0.5} \mathrm{Cd}_{0.5} \mathrm{~S} / \mathrm{ZnS}$ core/multishell QD structure was proposed by Xie and co-workers. ${ }^{[43]}$ As shown in Figure 2.15, the lattice parameters and energy levels of the multishell layer can be smoothly tuned from those of CdS to those of ZnS. In this way, better QD core surface passivation and higher PL QY can be achieved. 


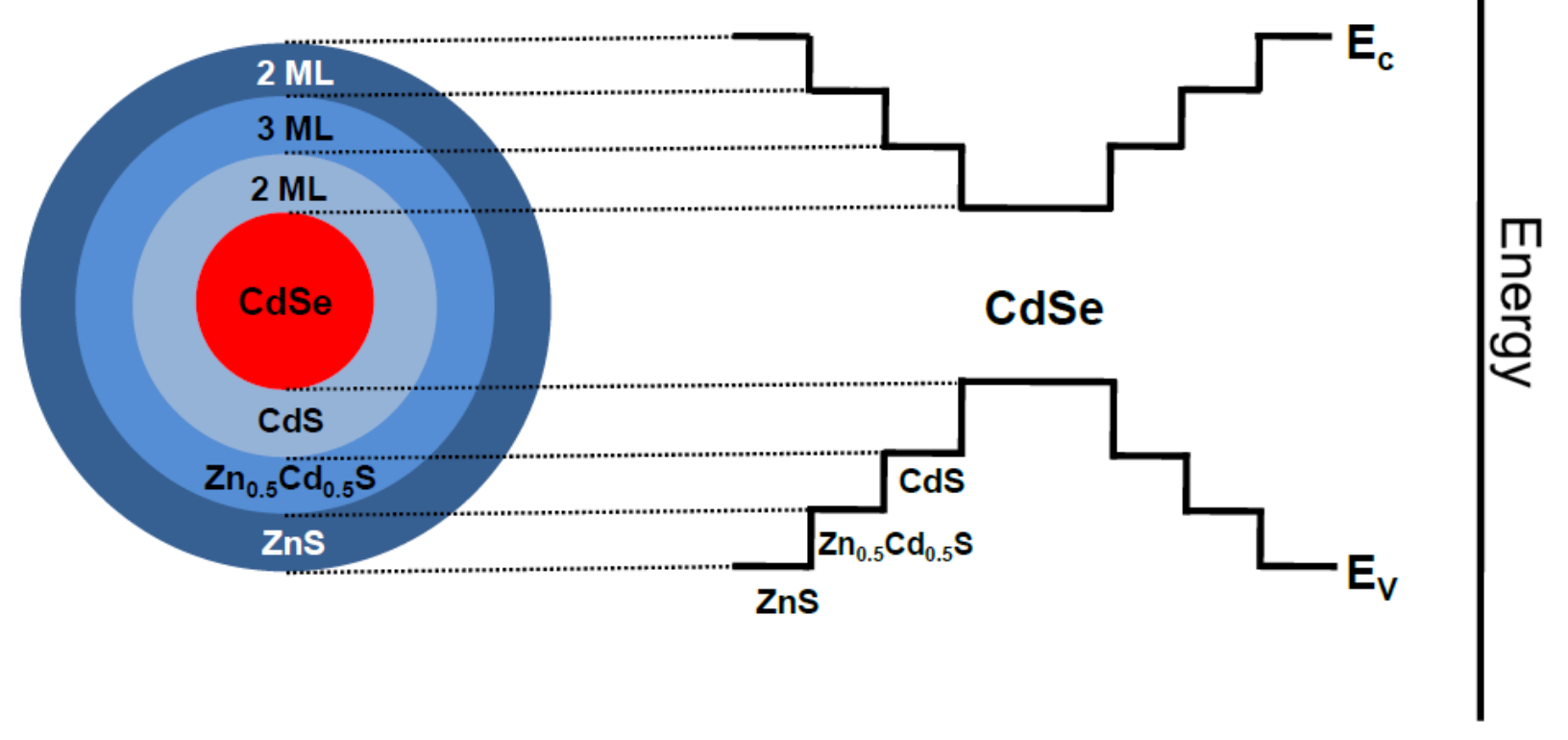

Figure 2.15 Schematic representation of band structure of $\mathrm{CdSe} / \mathrm{CdS} / \mathrm{Zn}_{0.5} \mathrm{Cd}_{0.5} \mathrm{~S} / \mathrm{ZnS}$ core/multishell QDs, demonstrating the gradual increase in the bandgap from the CdSe core to the outer ZnS shell

Again, we used a modified SILAR method to grow the CdS/ $\mathrm{Zn}_{0.5} \mathrm{Cd}_{0.5} \mathrm{~S} / \mathrm{ZnS}$ multishell on CdSe core QDs. First, precursors for the shell growth were prepared. The zinc precursor solution $(0.1 \mathrm{M})$ was prepared by dissolving $\mathrm{ZnO}(0.2849 \mathrm{~g})$ in OA $(9.1 \mathrm{~mL})$ and $\mathrm{ODE}(25.9 \mathrm{~mL})$ at $310^{\circ} \mathrm{C}$. The cadmium precursor solution $(0.1 \mathrm{M})$ was prepared by dissolving $\mathrm{CdO}(0.1541 \mathrm{~g})$ in $\mathrm{OA}(3 \mathrm{~mL})$ and $\mathrm{ODE}(9 \mathrm{~mL})$ at $250^{\circ} \mathrm{C}$. The $\mathrm{Zn} / \mathrm{Cd}$-precursor solution $(0.1 \mathrm{M})$ was prepared by dissolving $\mathrm{ZnO}(0.1221 \mathrm{~g})$ as well as $\mathrm{CdO}(0.1926 \mathrm{~g})$ in $\mathrm{OA}(7.8 \mathrm{~mL})$ and $\mathrm{ODE}(22.2 \mathrm{~mL})$ at $300{ }^{\circ} \mathrm{C}$. The sulfur precursor solution $(0.1 \mathrm{M})$ was prepared by dissolving sulfur in ODE at $180{ }^{\circ} \mathrm{C}$. All of the solutions were freshly prepared under $\mathrm{N}_{2}$ atmosphere. The Cd-, Zn-, and $\mathrm{Cd} / \mathrm{Zn}$-precursor solutions were kept at about $80{ }^{\circ} \mathrm{C}$, while the sulfur injection solution was allowed to cool to room temperature. For each shell growth, a calculated amount of a given precursor solution was injected with a syringe using standard air-free procedures.

A similar calculation method as used in CdSe/ZnS core/shell QDs synthesis (see details in Appendix 1) was used to calculate the amounts of precursor solutions. The average thicknesses 
of one ML of CdS and one ML of ZnS are $0.35 \mathrm{~nm}$ and $0.31 \mathrm{~nm}$, respectively. Therefore, the thickness of one monolayer of $\mathrm{Zn}_{0.5} \mathrm{Cd}_{0.5} \mathrm{~S}$ is assumed to be $0.33 \mathrm{~nm}$.

The structure of $\mathrm{CdSe} / \mathrm{CdS} / \mathrm{Zn}_{0.5} \mathrm{Cd}_{0.5} \mathrm{~S} / \mathrm{ZnS}$ core/multishell QDs for synthesis is schematically shown in Figure 2.15. Previously-synthesized CdSe core QDs (557 nm in $1^{\text {st }}$ excitonic absorption peak, $2.56 \mu \mathrm{mol}$ of particles) were mixed with $46 \mathrm{~mL}$ of ODE and $14.84 \mathrm{~g}$ of ODA in a three-necked flask. The mixture was heated to $220^{\circ} \mathrm{C}$ in $\mathrm{N}_{2}$ atmosphere, and the first ML of Cd-precursor (3.5mL) and S-precursor (3.5 mL) and the second ML of Cd-precursor (5 mL) and S-precursor (5 mL) were injected to the flask for the shell layer growth in the order of Cd-S at the interval of $10 \mathrm{~min}$ between two injections. Then, the growth temperature was adjusted to $240^{\circ} \mathrm{C}$. The third ML (Zn/Cd-precursor of $7 \mathrm{~mL}$ and S-precursor of $7 \mathrm{~mL}$ ), the fourth ML (Zn/Cd-precursor of $9 \mathrm{~mL}$ and S-precursor of $9 \mathrm{~mL}$ ), the fifth ML (Zn/Cd-precursor of 11.5 $\mathrm{mL}$ and S-precursor of $11.5 \mathrm{~mL}$ ), the sixth ML (Zn-precursor of $15 \mathrm{~mL}$ and S-precursor of 15 $\mathrm{mL}$ ), and the seventh ML (Zn-precursor of $17.8 \mathrm{~mL}$ and S-precursor of $17.8 \mathrm{~mL}$ ) were injected at the interval of $10 \mathrm{~min}$. The reaction mixture was allowed to cool to room temperature. This was followed by the QD extraction step ${ }^{[32]}$. Further purification of QDs was done by precipitating them with acetone or methanol. Highly pure QDs were obtained by repeating above purification procedure for 2-3 times. Aliquots of the solution were removed after each monolayer of shell growth with a syringe and tested for optical spectra. 


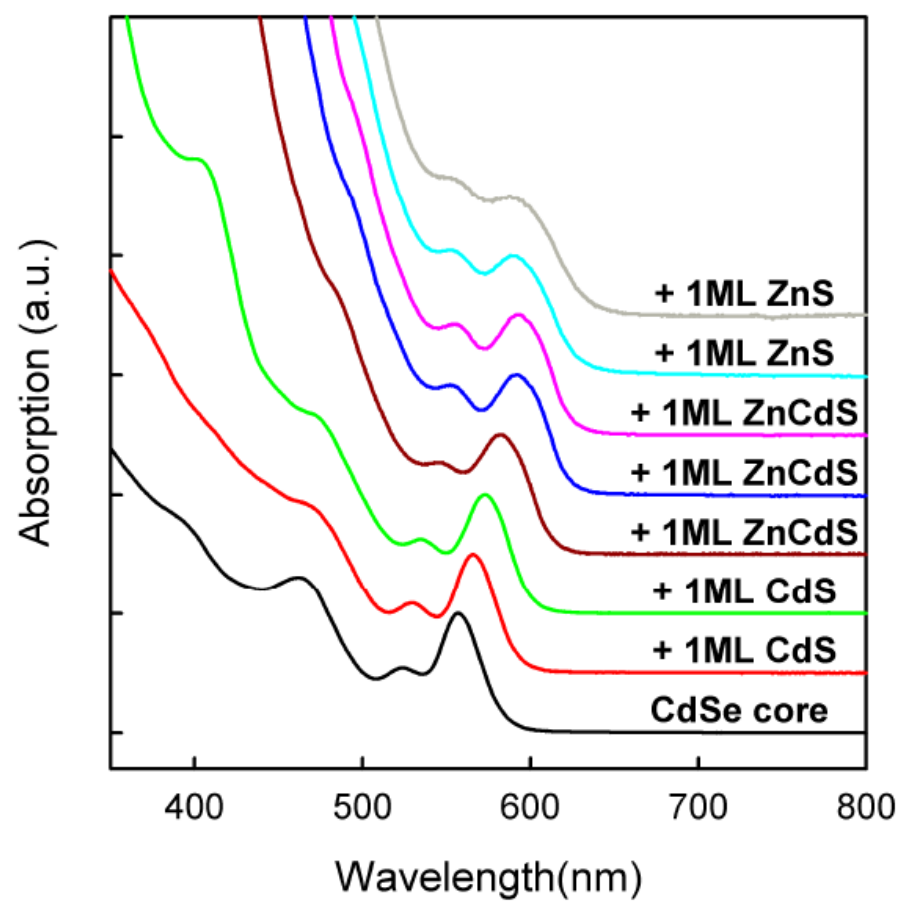

Figure 2.16 Evolution of the absorption spectra upon consecutive growth of the shells

The absorption spectra of QDs with different shell structures are shown in Figure 2.16. An evident red shift of the first excitonic absorption peak upon the shell growth can be seen from $557 \mathrm{~nm}$ for CdSe core QDs to $590 \mathrm{~nm}$ for the final core/multishell QDs. Hence, the red shift for $\mathrm{CdSe} / \mathrm{CdS} / \mathrm{Zn}_{0.5} \mathrm{Cd}_{0.5} \mathrm{~S} / \mathrm{ZnS}$ core/multishell QDs $(33 \mathrm{~nm}$ ) is much more pronounced than that of CdSe/ZnS core/shell QDs (20 nm) seen in Figure 2.12. A closer observation reveals that the growth of the first four shells (2MLs of CdS shell and 2MLs of ZnCdS shell) is mainly responsible for this red shift, whereas the growth of the remaining three shells (1ML of ZnCdS shell and 2MLs of ZnS shell) leads to minimal shift of the first excitonic absorption peak position. These results are attributed to the unique band alignment of the $\mathrm{CdSe} / \mathrm{CdS} / \mathrm{Zn}_{0.5} \mathrm{Cd}_{0.5} \mathrm{~S} / \mathrm{ZnS}$ core/multishell QDs. Compared to the ZnS shell, the CdS shell and ZnCdS alloy shell provide a weak confinement of electrons and holes. ${ }^{[43]}$ Therefore, the electronic wave function leakage into the first four shells may be significant, which results in a $33 \mathrm{~nm}$ red shift. After growing the final layers of ZnS, electrons and holes are better confined. 
The absorption spectrum of the final QDs retains the excitonic absorption features, indicating strong quantum confinement effects in the core/multishell QDs.

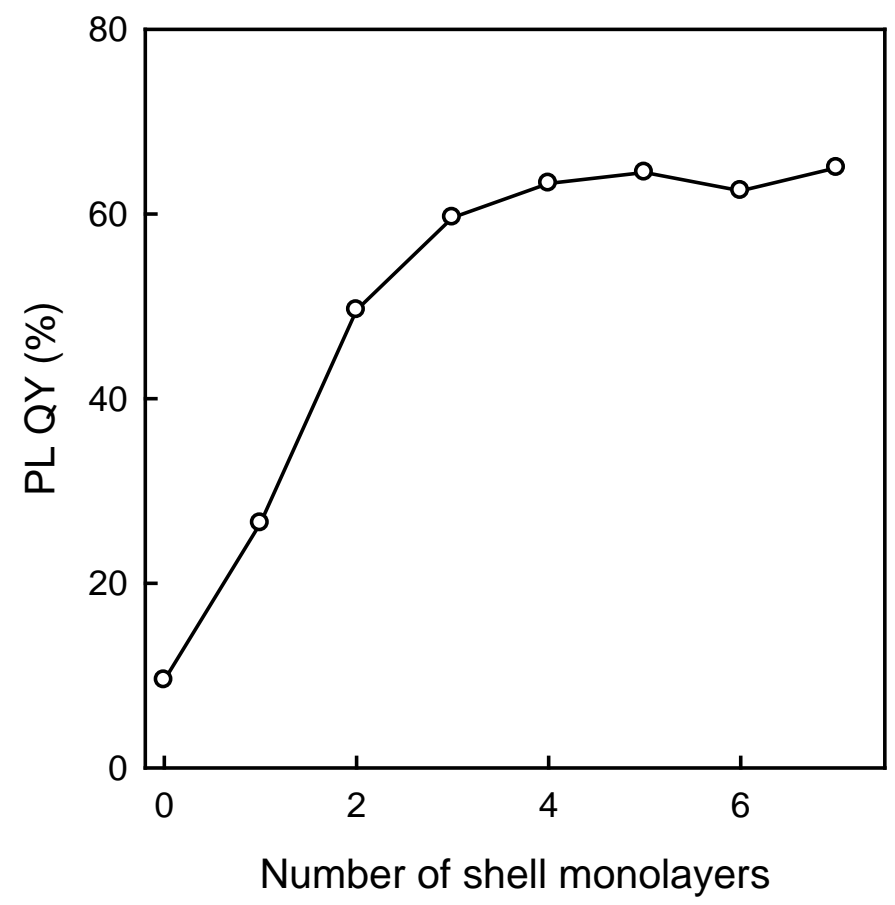

Figure 2.17 Evolution of the photoluminescence quantum yield of $\mathrm{CdSe} / \mathrm{CdS} / \mathbf{Z n}_{0.5} \mathrm{Cd}_{0.5} \mathrm{~S} / \mathrm{ZnS}$ core/multishell QDs with 2MLs of CdS shell, 3MLs of $\mathrm{Zn}_{0.5} \mathrm{Cd}_{0.5} \mathrm{~S}$ shell, and $2 \mathrm{MLs}$ of $\mathrm{ZnS}$ shell

The evolution of the PL QY of CdSe/CdS/ $\mathrm{Zn}_{0.5} \mathrm{Cd}_{0.5} \mathrm{~S} / \mathrm{ZnS}$ core/multishell QDs is shown in Figure 2.17. The QY of the CdSe QDs is raised from 10\% to $60 \%$ upon coverage of 2 MLs of CdS shell and $1 \mathrm{ML}$ of $\mathrm{Zn}_{0.5} \mathrm{Cd}_{0.5} \mathrm{~S}$ shell by the SILAR method. The QY of QDs is further increased to $\sim 65 \%$ after additional growth of $2 \mathrm{MLs}$ of $\mathrm{Zn}_{0.5} \mathrm{Cd}_{0.5} \mathrm{~S}$ shell and $2 \mathrm{ML}$ of $\mathrm{ZnS}$ shell. Figure 2.18 shows the comparison of the absorption and PL spectra of CdSe core and CdSe/CdS/Zn ${ }_{0.5} \mathrm{Cd}_{0.5} \mathrm{~S} / \mathrm{ZnS}$ core/multishell QDs. The core/multishell QDs achieve a high PL QY as well as high stability mainly due to the use of a graded shell structure. The result shows that more effective and stable surface passivation of CdSe core can be achieved by the multishell than a simple ZnS shell. 


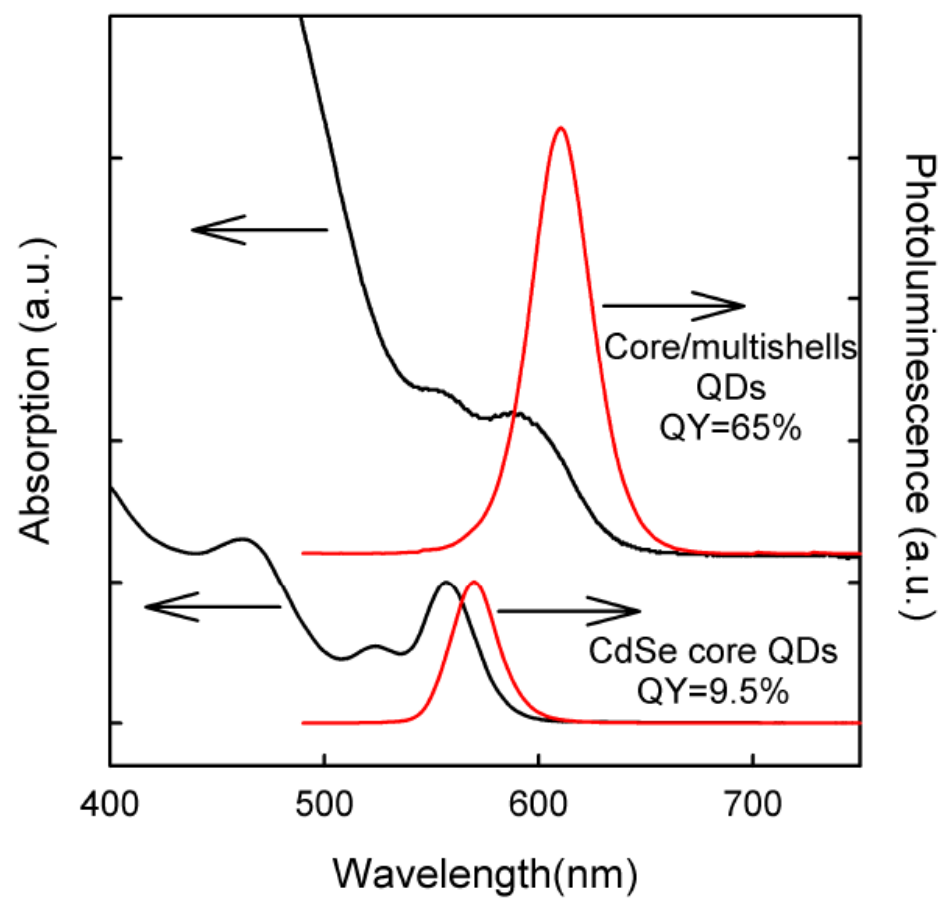

Figure 2.18 PL spectra and absorption spectra of red-emitting CdSe core QDs and CdSe/CdS/Zn ${ }_{0.5} C d_{0.5} S / Z n S$ core/multishell QDs (as-prepared)

\subsection{Conclusions}

An in-house capability of colloidal QD synthesis by the hot-injection method was developed. Through tuning reaction time, the absorption spectrum and PL emission peak of QDs can be tuned over a wide range. High-quality CdSe, CdS and CdTe core QDs with sharp excitonic absorption features and narrow PL bandwidths were synthesized. Furthermore, CdSe/ZnS core/shell QDs were synthesized via the SILAR method. The highest PL QY 50\% was obtained after the growth of 3 monolayers of ZnS shell. Finally, in order to reduce the interfacial defects so as to further improve the PL QY, CdSe QDs with a graded $\mathrm{CdS} / \mathrm{Zn}_{0.5} \mathrm{Cd}_{0.5} \mathrm{~S} / \mathrm{ZnS}$ multishell were synthesized. A PL QY $65 \%$ was achieved. These results are comparable to the best data reported by other research groups. ${ }^{[21,28,32,43,65-66]}$ This work lays an important groundwork for developing QD-based energy devices described in Chapter 3-5. 


\section{Chapter 3 Development of High-Efficiency CdSe/ZnS QD-}

\section{LEDs}

Recently, Sun and co-workers reported high-performance QD-LEDs with saturated red, orange, yellow, and green emissions, all utilizing CdSe/ZnS core/shell QDs. ${ }^{[29]}$ As compared to the previously reported QD-LEDs, their devices included a buffer layer of PEDOT:PSS between indium tin oxide (ITO) electrode and hole transport layer (HTL) to improve the hole injection and reduce the surface roughness of the anode to obtain stable and pin-hole-free electrical conduction across the device. ${ }^{[67]}$ Poly-TPD was used as the HTL in consideration of the fact that its highest occupied molecular orbital (HOMO) level is $5.2 \mathrm{eV}$, which is very close to the work function of the ITO/PEDOT:PSS anode, and also because it possesses an excellent hole-transport capability $^{[68]}$. Moreover, poly-TPD has proven to be a good resistor to nonpolar organic solvents such as toluene and hexane ${ }^{[69]}$, which are often used to dissolve colloidal QDs. In addition, because poly-TPD can be cured at a low temperature, a PEDOT:PSS buffer layer can be used as the HIL. $\mathrm{Alq}_{3}$ was chosen as the ETL because of its good electron-transport capability ${ }^{[70]}$ and its interfacial phase compatibility with the QD layer ${ }^{[54]}$. Even more important, they found that the thickness of the QD layer determines the efficiency and luminance of QD-LEDs and should be carefully optimized for each color, depending on the size and structure of QDs. Uniform and defect-free EL emission from the QDs over a large surface area $(1.5 \mathrm{~cm} \times 2.5 \mathrm{~cm})$ was displayed. The result shows good promise for the application of QD-LEDs in large-area displays.

Inspired by their work, we employ this kind of QD-LED structure as our baseline QDLED structure. In this chapter, we first focus on structure optimization of green CdSe/ZnS QDLEDs via tailoring the thickness of the QD emitting layer by tuninig the CdSe/ZnS QD solution concentration during spin-coating. We then incorporate a phosphorescent donor layer into the 
device structure to enhance exciton energy transfer in order to achieve performance improvement of the QD-LEDs. ${ }^{[71]}$

\subsection{Structure Optimization}

The QD-LEDs were fabricated on glass substrates with pre-patterned ITO (sheet resistance $\sim 15 \Omega / \square$ ). The substrates were sequentially cleaned in acetone, ethanol, and de-ionized water, and exposed to oxygen plasma for 5 min. A $40 \mathrm{~nm}$ PEDOT:PSS (Aldrich 560596) layer was first spin-coated at a spin rate of $5000 \mathrm{rpm}$ for $30 \mathrm{~s}$ and dried at $150{ }^{\circ} \mathrm{C}$ under $\mathrm{N}_{2}$. A $40 \mathrm{~nm}$ poly-TPD (ADS 254BE) layer was then spin-coated from a chloroform solution $(6 \mathrm{mg} / \mathrm{mL})$ on top of the PEDOT:PSS layer at $2500 \mathrm{rpm}$ for $30 \mathrm{~s}$, and was annealed at $110{ }^{\circ} \mathrm{C}$ in $\mathrm{N}_{2}$ for $30 \mathrm{~min}$. Green CdSe/ZnS QDs were deposited on the surface of poly-TPD by spin-coating from a hexane solution at a rate of $3000 \mathrm{rpm}$ for $1 \mathrm{~min}$. The samples were then transferred from a glovebox to a high-vacuum thermal evaporation system. A multilayer organic structure was deposited onto the QD layer by evaporation deposition at rates of $\sim 0.1 \mathrm{~nm} / \mathrm{s}$ under $3 \times 10^{-7}$ Torr. The structure consisted of a $15 \mathrm{~nm}$ BCP hole blocking layer (HBL), and a $20 \mathrm{~nm} \mathrm{Alq}$ electron transport layer (ETL). Finally, a $0.5 \mathrm{~nm} \mathrm{LiF}$ and $120 \mathrm{~nm} \mathrm{Al} \mathrm{cathode} \mathrm{were} \mathrm{deposited} \mathrm{atop} \mathrm{the} \mathrm{organic} \mathrm{film}$ through a shadow mask. Four different QD-LEDs were fabricated at one round, using four different QD concentration solutions to deposit the QD emission layer $(1 \mathrm{mg} / \mathrm{mL}, 3 \mathrm{mg} / \mathrm{mL}, 5$ $\mathrm{mg} / \mathrm{mL}$ and $10 \mathrm{mg} / \mathrm{mL}$ ). The corresponding QD layer thickness was estimated to be 0.5 MLs, 1.6 MLs, 3 MLs and 7 MLs, respectively, based on the measured optical properties of the QD films. ${ }^{[29]}$ All the QD-LEDs had an emitting area of $0.09 \mathrm{~cm}^{2}$. Figure 3.1 shows the Trovato evaporation system integrated with MBraun glovebox for fabrication of QD-LEDs.

The QD-LEDs were encapsulated with glass lids in $\mathrm{N}_{2}$ and characterized in air at room temperature. The electrical characteristics were measured using an Agilent 4156C semiconductor analyzer. The EL spectra were recorded as a function of injection current density in the range of $0.1-200 \mathrm{~mA} / \mathrm{cm}^{2}$ using a silicon photodiode-array fiber-optic spectrometer. To calculate EQE, 
the QD-LEDs were placed directly on the surface of a calibrated silicon photodetector and all emitted photons from the glass side were captured.

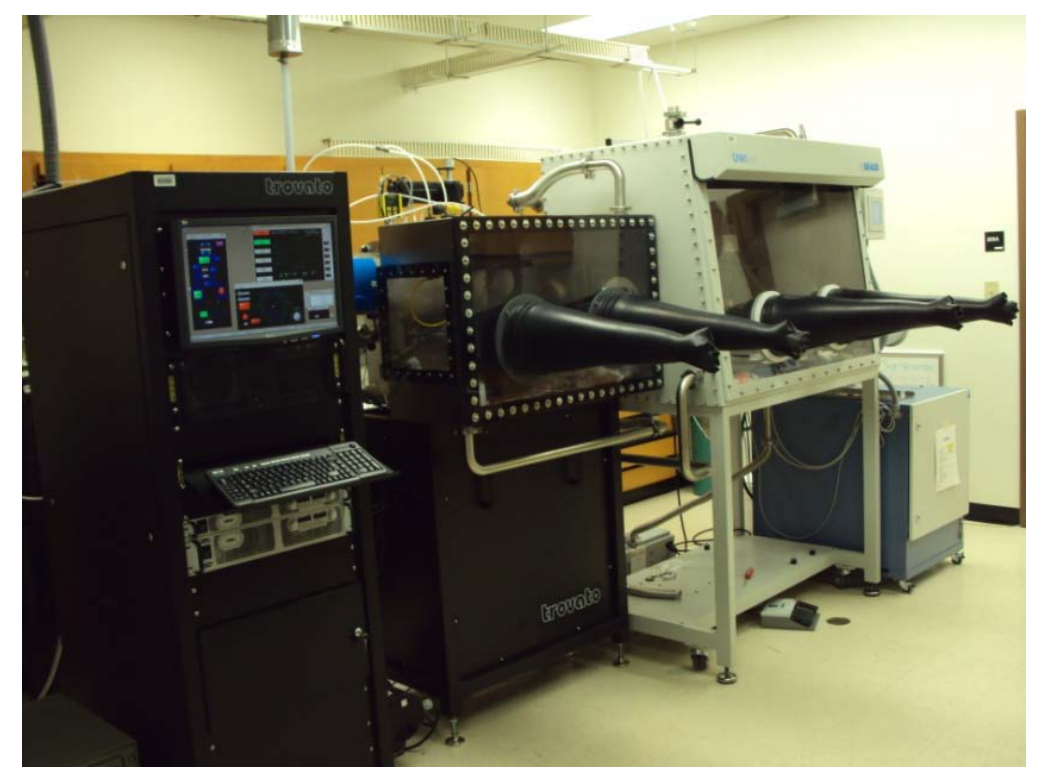

Figure 3.1 Trovato evaporation system integrated with MBraun glovebox for fabrication of QD-LEDs

The proposed CdSe/ZnS QD-LEDs structure and energy diagram are schematically shown in Figure 3.2. PEDOT:PSS layer was used as hole injection layer. The poly-TPD was chosen as the HTL because it possesses an intrinsic resistance to nonpolar organic solvents and a high hole mobility with effective electron blocking properties. Green CdS/ZnS core/shell QDs were used as emission layer. BCP and Alq3 were used as HBL and ETL, respectively. 
a)

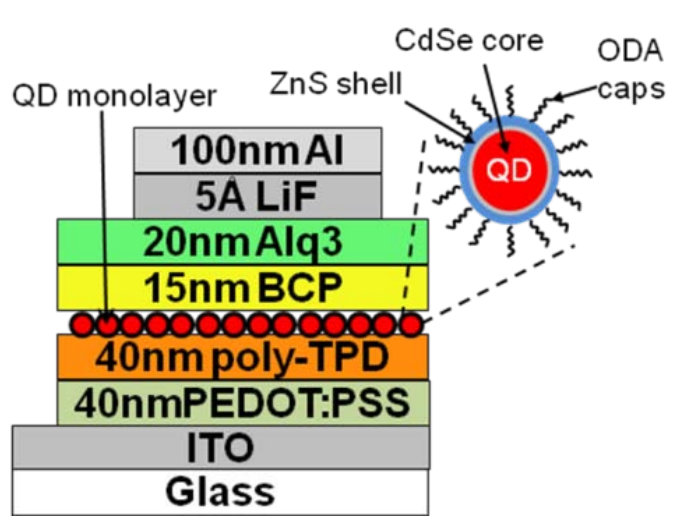

b)

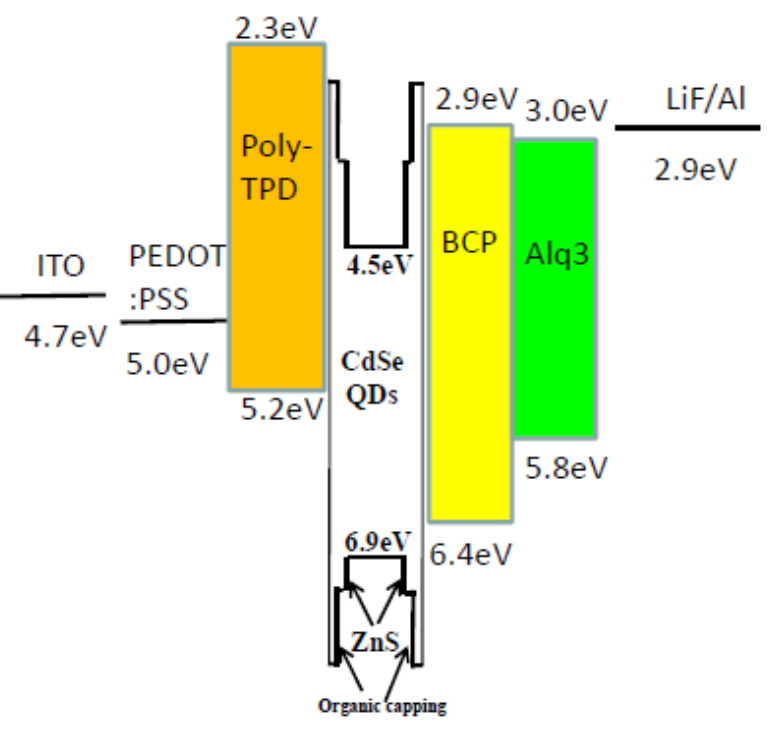

Figure 3.2 a) The cross-section and b) the energy diagram of the QD-LEDs

To optimize the QD layer thickness, the layer was prepared by spin-coating QD solutions of different concentrations in the range of $1-10 \mathrm{mg} / \mathrm{ml}$, while all other experimental conditions in the test devices were kept constant. Figure 3.3 (a) shows the EL spectra of green QD-LEDs prepared from 1, 3, 5 and $10 \mathrm{mg} / \mathrm{ml}$ QD dispersions in hexane. The QD-LED prepared from a 3 $\mathrm{mg} / \mathrm{ml}$ solution exhibits the highest light intensity at the same current density. The corresponding layer thickness was estimated to be 1.6 MLs based on the measured optical properties of the QD films. Figure 3.3 (b) demonstrates the normalized EL spectra of green QD-LEDs prepared from 3 mg/ml QD solution. Pure QDs emitting peak without any organic emitting peaks is realized from $0.01 \mathrm{~mA}$ to $20 \mathrm{~mA}$. 

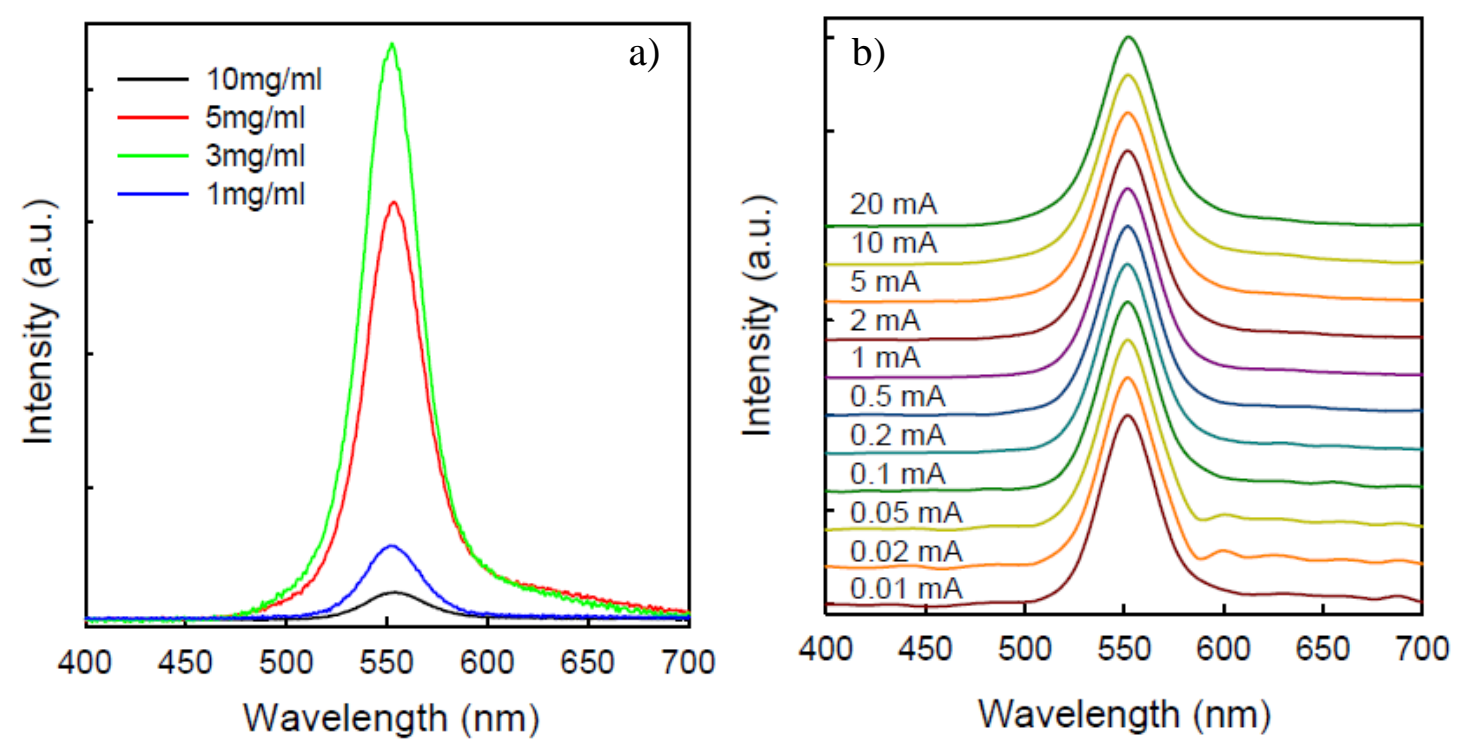

Figure 3.3 EL spectra of green QD-LEDs

a) EL spectra of green QD-LEDs prepared from 1-10 mg/ml QD solutions recording at $1 \mathrm{~mA}$. b) Normalized EL spectra of green QD-LEDs prepared from $3 \mathrm{mg} / \mathrm{ml}$ QD solution

The QD-LED prepared from a $3 \mathrm{mg} / \mathrm{ml}$ solution shows a moderate turn-on voltage of $\sim 4$ $\mathrm{V}$ (Figure 3.4). The highest EQE of green QD-LEDs we have obtained are $\sim 0.2 \%$, which are comparable with the performance of similar QD-LEDs reported in the literature. ${ }^{[53]}$ It is clearly seen from Figure 3.4 that both the turn-on voltage and series resistance of the QD-LEDs increase with as the thickness of the QD layer increases. The QD-LED with QDs spin coated from a 1 $\mathrm{mg} / \mathrm{ml}$ solution has the lowest forward voltage, but its EL is 5 times lower, presumably due to a partial surface coverage by less than one monolayer of QDs. 


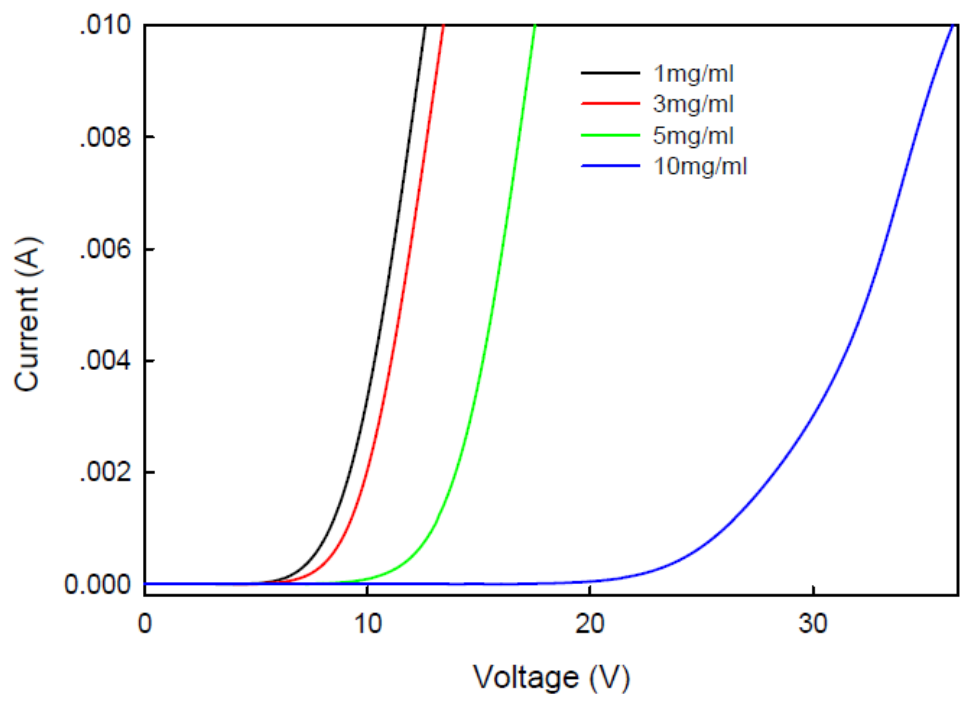

Figure 3.4 I-V Characteristics of CdSe/ZnS QD-LEDs with different concentration of QD solutions

\subsection{Performance Enhancement with Phosphorescent Donors}

The unique properties of colloidal inorganic QDs, including high luminescence efficiency, broad wavelength tunability, saturated color emission, and good material stability, make them very attractive for developing novel hybrid LEDs. ${ }^{[11,29,52-54]}$ Colloidal QDs also offer opportunities for inexpensive device fabrication by solution processing, and are thus a promising class of materials suitable for low-cost and large-area manufacturing. ${ }^{[16]}$ Despite that QDs exhibit very high photoluminescence (PL) quantum yields given appropriate surface functionalization, the EL of hybrid LEDs with QD luminophores embedded in organic or polymeric heterostructures (referred to as QD-LEDs herein) remains very low. ${ }^{[11,29,52-54]}$ The highest reported EQE of QD-LEDs, achieved by using closely-packed single monolayer of QDs, is only $\sim 2.7 \%,{ }^{[55]}$ far below those of state-of-the-art all organic or polymer LEDs. ${ }^{[62]}$

Excitons in QDs may be generated by direct injection of carriers from organic or polymeric charge transport layers. In this case, the EQE of QD-LEDs is mainly limited by inefficient and imbalanced charge injection caused by two material factors: (i) Most QDs have a low-lying valence band much lower than the highest occupied molecular orbital level of hole 
transport materials. ${ }^{[72]}$ As a result, the energy barriers for hole injection into QDs are large, typically greater than $1 \mathrm{eV}$, leading to poor hole injection as compared to electron injection; (ii) Colloidal QDs are passivated by insulating and relatively bulky organic ligands introduced onto the QD surfaces for dispersion during the synthesis. This organic capping layer increases the interparticle spacing and interfacial energy barriers, and thus causes poor QD-QD charge transport and carrier injection. ${ }^{[33]}$ In addition to direct injection, resonant energy transfer is another mechanism by which excitons may form in QDs. In this process, injected carriers create excitons on organic molecules surrounding the QDs, and the excitons then transfer their energy to QDs via Förster or Dexter processes. ${ }^{[73]}$ Due to poor injection of carriers (holes particularly) into QDs, energy transfer has often been found to play a dominant role in the EL process of QDLEDs. $^{[55]}$

In typical QD-LEDs, the QD emitting layer (EML) is sandwiched between hole and electron transport layers (HTL and ETL) of fluorescent materials. The lifetime of excitons generated in these materials is short due to fast radiative and nonradiative decay, which may compete favorably with energy transfer to QDs. This hypothesis is supported by the finding by Clapp et al., ${ }^{[74]}$ who observed no apparent energy transfer to CdSe/ZnS QDs from fluorescent dyes attached to protein molecules self-assembled on the QD surfaces. In contrast, phosphorescent materials have much longer exciton lifetimes, and thus may act as more effective exciton energy donors transferring energy to QD acceptors. ${ }^{[73]}$ In particular, heavy metal phosphorescent materials such as Ir(III) complexes are efficient triplet exciton harvesters when they are doped in a fluorescent host. In the host-guest system, singlet excitons formed on the host molecules can transfer energy to guest singlets which then undergo rapid intersystem crossing (ISC) to the triplet state enabled by heavy atom-induced strong spin-orbit coupling. So both singlet and triplet excitons are harnessed for light emission. ${ }^{[62]}$ Resonant energy transfer from Ir(III) complexes to CdSe/ZnS QDs has been observed through optical excitaion. ${ }^{[73]}$ However, such energy transfer has not been specifically observed and utilized in electrically-injected QD- 
LEDs. In this section, we investigate a new QD-LED structure which integrates green CdSe/ZnS core/shell QDs with blue phosphorescent dye Bis(4,6-difluorophenylpyridinatoN,C2)picolinatoiridium (FIrpic), and uses the latter as an efficient exciton harvester and energy donor. Spectral overlap between the FIrpic emission and QD absorption shown in Figure 3.5 suggests the probability of efficient exciton energy transfer from FIrpic to the green QDs. By adjusting the donor concentration and donor-acceptor distance, complete exciton energy transfer from FIrpic to the QDs has been realized, leading to 2.5-fold EL enhancement over QD-LEDs without FIrpic donors.

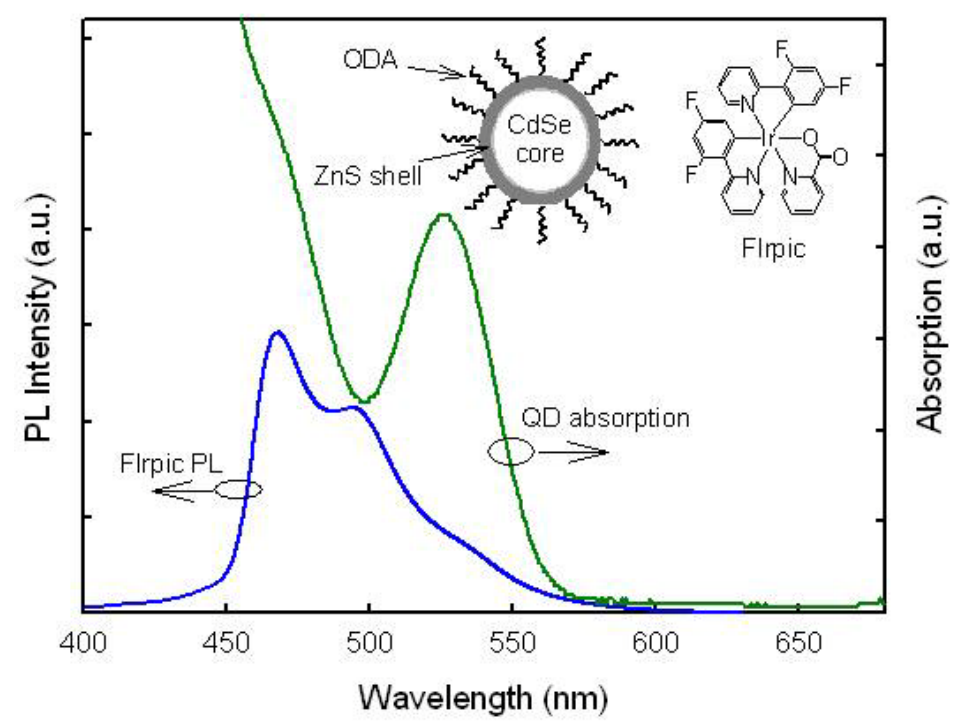

Figure 3.5 Absorption spectrum of green CdSe/ZnS QDs in hexane shown along with PL spectrum of FIrpic in chloroform

The spectral overlap suggests the probability of efficient exciton energy transfer from FIrpic to the QDs. The inset shows the schematic drawings of a green QD and the chemical structure of FIrpic.

A similar procedure as section 3.1 was use to fabrication QD-LEDs from a green CdSe/ZnS core/shell QDs solution in hexane (3 mg/mL), while the only difference is that a CBP layer (3-17 nm) doped with FIrpic (0-30wt\%) was evaporated between the QD layer and the BCP layer (Figure 3.6). 
(a)

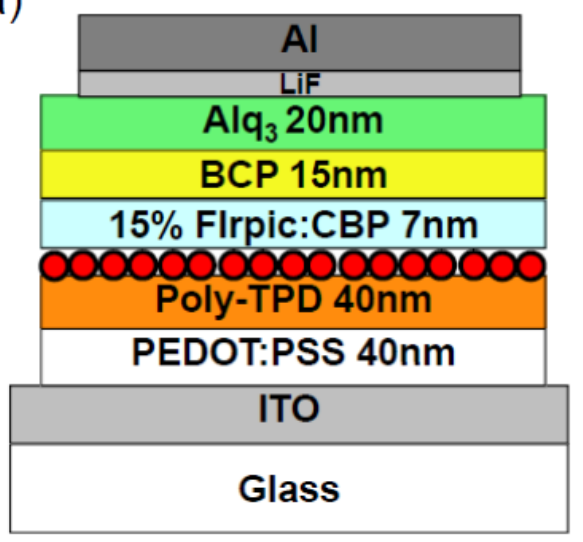

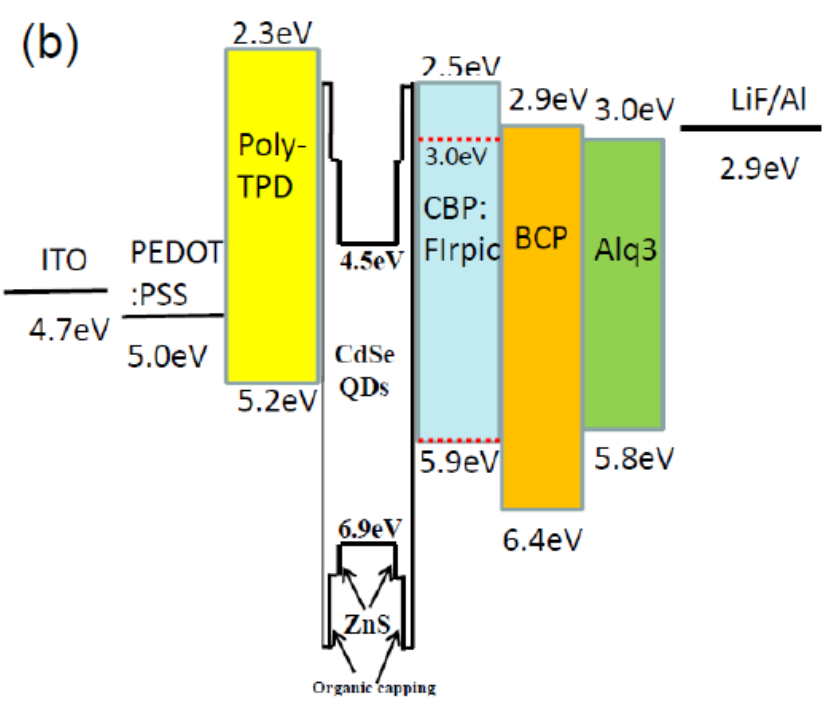

Figure 3.6 (a) Layer structure of a CdSe/ZnS QD-LED with a FIrpic-doped CBP energy-donor layer. (b) The energy diagram of the QD-LED

The QD-LED structure consists of a $40 \mathrm{~nm}$ poly(3,4-ethylenedioxythiophene) poly(styrenesulfonate) (PEDOT:PSS) spin-coated on ITO/glass substrates, a $40 \mathrm{~nm}$ poly-(N,N'bis(4-butylphenyl)-N,N'-bis(phenyl)benzidine) (poly-TPD) HTL, a green CdSe/ZnS QD EML, a $15 \mathrm{~nm}$ 2,9-dimethyl-4,7-diphenylphenanthroline (BCP) hole blocking layer (HBL), and a $20 \mathrm{~nm}$ tris-(8-hydroxyquinoline) aluminum $\left(\mathrm{Alq}_{3}\right)$. $\mathrm{Al}(120 \mathrm{~nm})$ is used as the cathode, whose overlap with the ITO anode defines an emitting area of $0.09 \mathrm{~cm}^{-2}$. As shown in Figure 3.6, a 4,4'-N, N'dicarbazole-biphenyl (CBP) layer doped with FIrpic is inserted between the QD EML and BCP HBL to capture overinjected holes. It is expected that excitons generated in the CBP layer would transfer energy back to the QDs, enhancing QD light emission. 

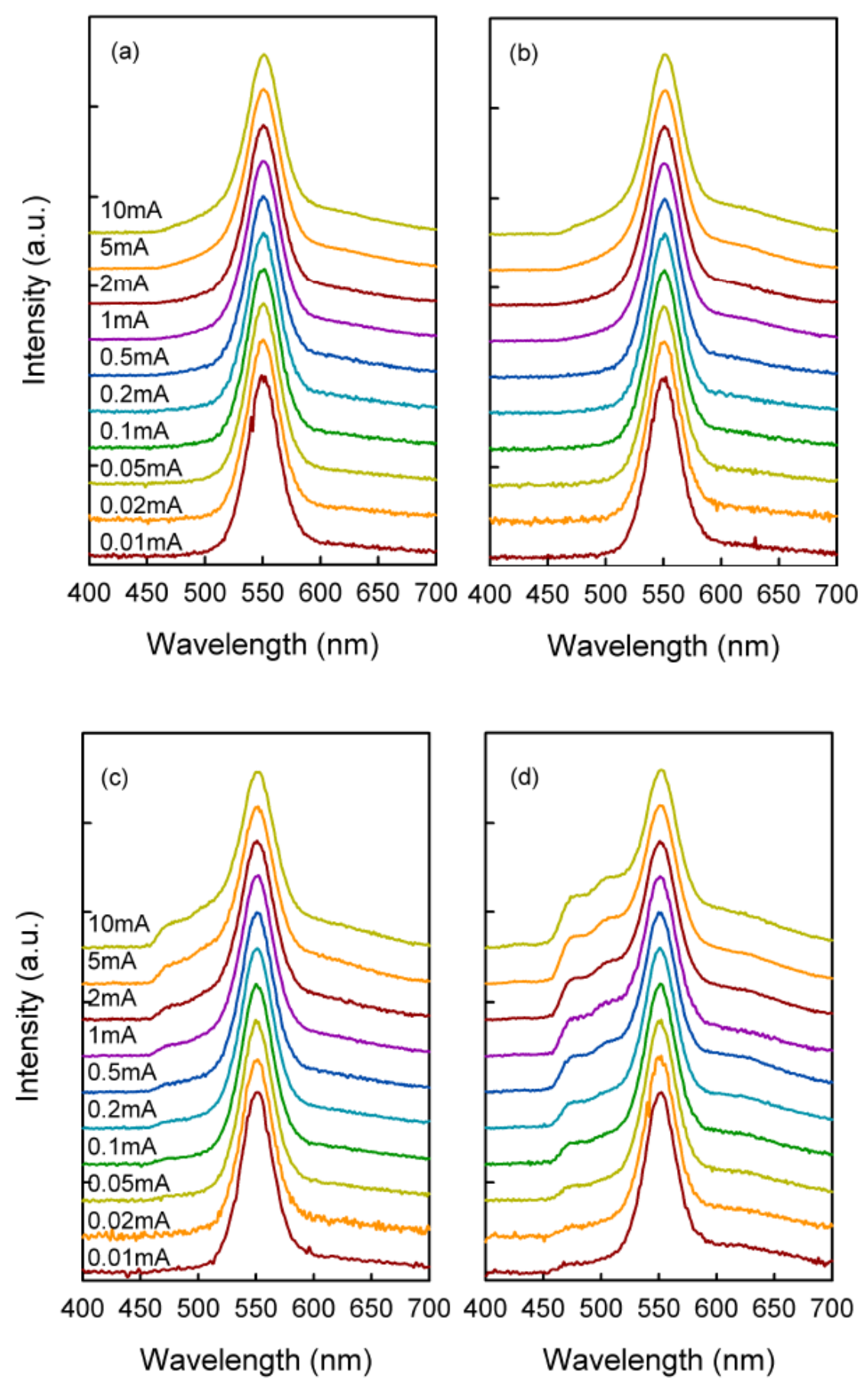

Figure 3.7 Normalized EL spectra of green CdSe/ZnS QD-LEDs

Normalized EL spectra of green QD-LEDs with a (a) $6 \mathrm{~nm}$, (b) $8 \mathrm{~nm}$, (c) $10 \mathrm{~nm}$, or (d) $17 \mathrm{~nm}$ CBP donor layer doped with 15\% FIrpic, at various injection currents.

Figure 3.7 shows the normalized EL spectra of QD-LEDs with $6 \mathrm{~nm}, 8 \mathrm{~nm}, 10 \mathrm{~nm}$ and 17 
nm CBP doped with 15wt\% FIrpic with increasing injection current. The QD-LED with $6 \mathrm{~nm}$ FIrpic-doped CBP shows the typical green EL solely from QDs over the entire current range. At low injection currents $(<2 \mathrm{~mA}$ ), the QD-LED with $8 \mathrm{~nm}$ FIrpic-CBP has similar green QD EL spectra. However, above $2 \mathrm{~mA}$, a tiny blue-cyan emission emerges at high-energy side of the main QDs EL peak, which is attributed to phosphorescent emission from FIrpic. And this blue band is more noticeable for the QD-LED with 10nm FIrpic-CBP, especially under the injection current larger than $0.1 \mathrm{~mA}$. For the QD-LED with $17 \mathrm{~nm}$ FIrpic:CBP, blue-cyan emission from FIrpic in addition to green EL from the QDs is clearly seen at all currents and their intensity ratio increases with increasing current. Hence, for the QD-LED with thicker FIrpic:CBP layer (>10 $\mathrm{nm}$ ), it is evident that at elevated injection levels, a significant amount of excitons can be radiatively recombined within the FIrpic:CBP layer, rather than energy transferred to the QDs layer. Furthermore, the green peak intensities of these four QD-LEDs are found to be significantly increased compared to a control QD-LED without a FIrpic:CBP layer, whereas FIrpic emission is completely or partly quenched compared to an organic LED with a FIrpic:CBP EML. These results imply that all excitons generated on FIrpic molecules in the $6 \mathrm{~nm}$ or $8 \mathrm{~nm}$ CBP layer transfer their energy to the QDs, leading to enhanced QD EL, except for the $8 \mathrm{~nm}$ CBP layer under high injection current (>2 mA). Whereas, energy transfer from the $10 \mathrm{~nm}$ or 17 nm FIrpic:CBP layer is incomplete. 

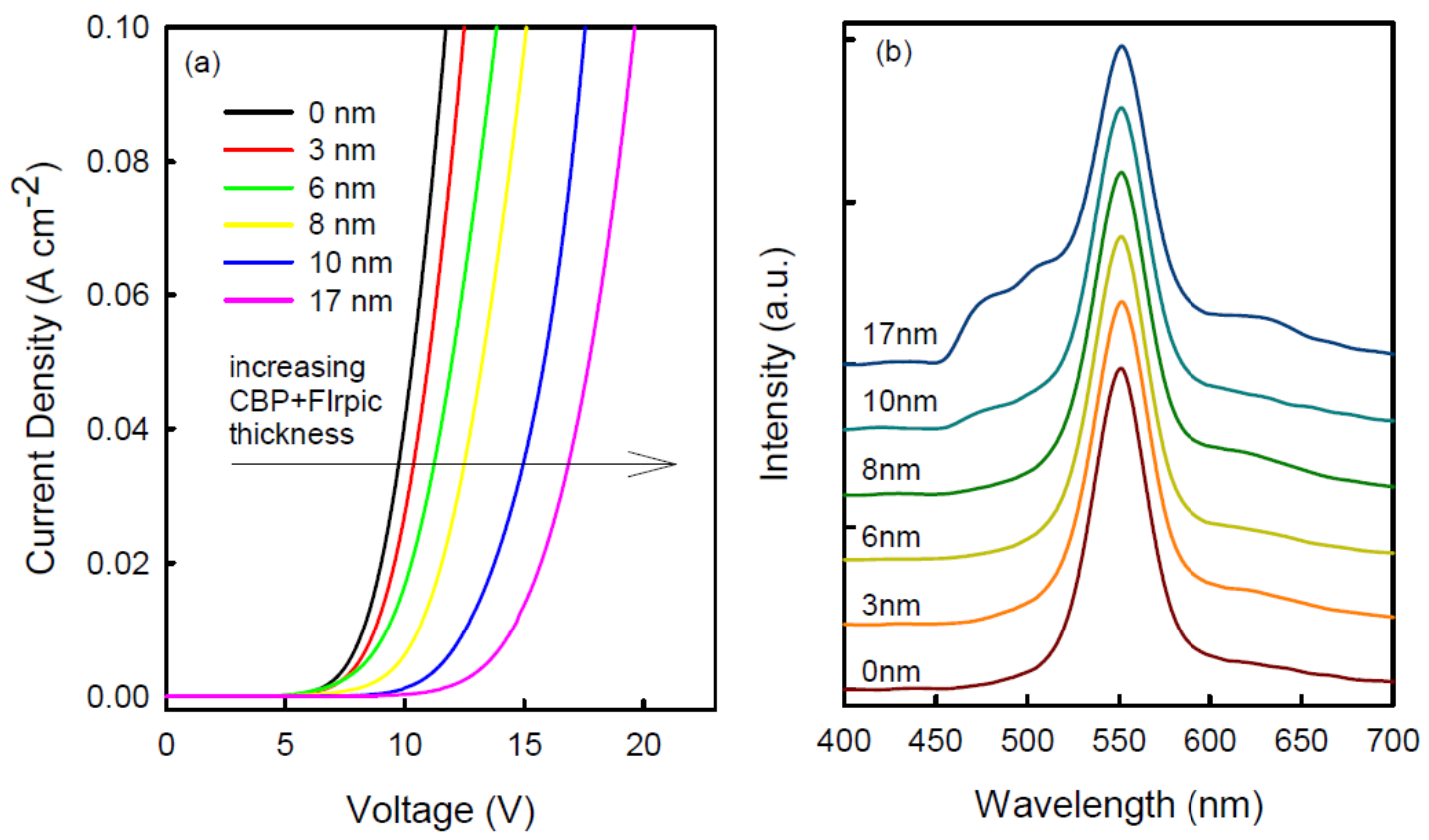

Figure 3.8 (a) I-V characteristics and (b) normalized EL spectra, recorded at $10 \mathrm{~mA} / \mathrm{cm} 2$ of green QD-LEDs with a CBP donor layer of 3-17 $\mathrm{nm}$ doped with $15 \%$ FIrpic

In order to gain more insight into the energy transfer mechanism, we varied the thickness of the FIrpic:CBP layer from $3 \mathrm{~nm}$ to $17 \mathrm{~nm}$ and the FIrpic doping concentration from 2\% to 30\%. As shown in Figure 3.8 (a), QD-LEDs with FIrpic:CBP layer have similar I-V characteristics, except that the forward voltage increases gradually with increasing FIrpic:CBP thickness. The voltage at $10 \mathrm{~mA} / \mathrm{cm}^{2}$ varies from 8.8 to $14.5 \mathrm{~V}$ as the thickness is increased from 3 to $17 \mathrm{~nm}$, as compared to $8.2 \mathrm{~V}$ for the control QD-LED without the FIrpic-doped CBP layer. Evidently an extra bias must be applied to maintain the current transport across the FIrpic:CBP layer due to slow carrier mobilities. ${ }^{[75]}$ The normalized EL spectra of these QD-LEDs at 10 $\mathrm{mA} / \mathrm{cm}^{2}$ are depicted in Figure 3.8 (b). The QD-LEDs with FIrpic:CBP layer thinner than $8 \mathrm{~nm}$ exhibit a single green-emitting peak at 550nm, attributing to the typical QDs emission. In contrast, for the QD-LEDs with the FIrpic:CBP layer thicker than $10 \mathrm{~nm}$, blue emission from FIrpic in addition to the QD emission is clearly seen, and their intensity ratio increase with increasing FIrpic:CBP layer thickness. Thus, the critical CBP layer thickness is between 8 and 10 nm. All excitons formed on FIrpic molecules located within the critical distance from the 
CBP/QD interface can completely transfer their energy to QDs, whereas beyond 8-10 nm, excitons on FIrpic molecules may decay radiatively. It has also been identified that QD-LEDs with $6 \mathrm{~nm}$ CBP doped with 2-30\% FIrpic emit green light only at all currents. This suggests that, regardless of the exciton density, excitons within the transfer distance exhibit complete longrange energy transfer due to the fact that the transfer rate is much faster than phosphorescent decay.

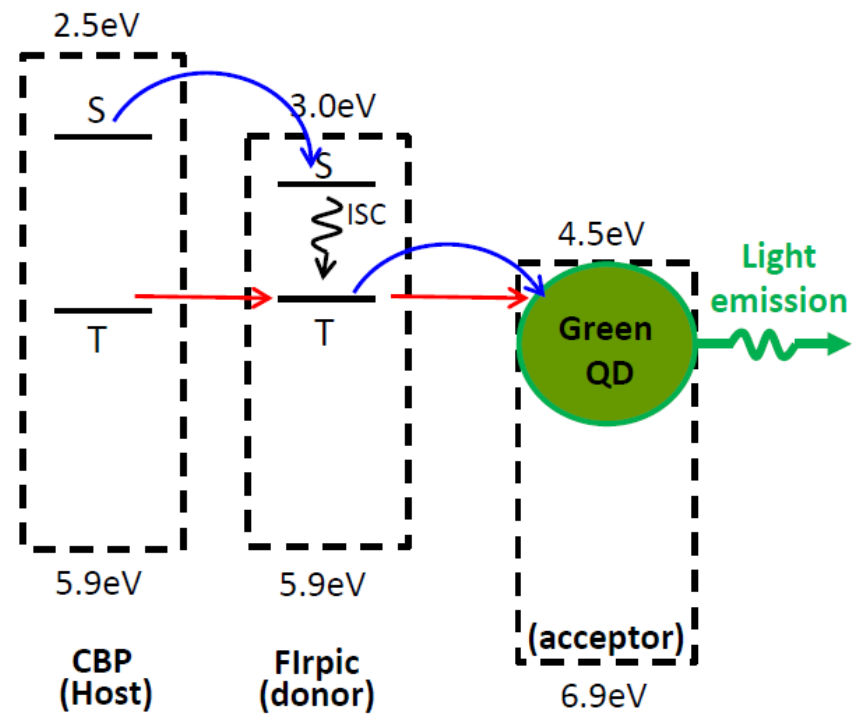

Figure 3.9 Energy diagram and proposed mechanisms of QD-LEDs

Energy diagram and proposed mechanisms of energy transfer from FIrpic molecules to CdSe/ZnS QDs in a hybrid LED (blue arrows: Förster transfer; red arrows: Dexter transfer).

The possible mechanisms of energy transfer in the QD/FIrpic:CBP hybrid system are schematically illustrated in Figure 3.9. In the control device, excitons are formed directly in QDs or on fluorescent molecules near the QD/organics interfaces then partially transfer their energy to QDs. When a CBP layer doped with phosphorescent FIrpic is added, it acts as a reservoir for holes which are not captured by QDs, and thus an exciton generation region. Singlet excitons on CBP molecules can resonantly transfer their energy to singlets on FIrpic molecules and then convert into the triplet state via efficient intersystem crossing. In the meantime, triplet excitons on CBP molecules can directly transfer energy to triplets on FIrpic by the Dexter mechanism. 
Therefore, FIrpic molecules behave as effective triplet exciton harvesters. The long lifetime of FIrpic triplets allows complete exciton energy transfer to QDs within a certain range. For FIrpic molecules close to the QD layer, Dexter process, ${ }^{[76]}$ a short range process which requires overlap of the molecular orbits of adjacent molecules, may play an important role. The transfer rate falls off exponentially with the donor-acceptor separation $R_{D A}$, i.e. $k_{D} \propto \exp \left(-2 R_{D A} / L\right)$, where $L$ is the sum of the van-der Waals radii of the donor and acceptor. Therefore, its contribution to the overall energy transfer is likely limited to the first monolayer of FIrpic:CBP adjacent to the QD layer. In the meanwhile, exciton energy may be transferred resonantly over a much longer distance via the Förster mechanism. ${ }^{[77]}$ The Förster radius $R_{F}$, a characteristic donor-acceptor distance at which the transfer rate is equal to the donor exciton decay rate, can be calculated from the overlap between the FIrpic emission and QD absorption spectra shown in Figure 3.5: ${ }^{[77]}$

$$
R_{F}^{6}=\frac{9}{8 \pi} \frac{c^{4}}{n^{4}} \kappa^{2} \eta_{D} \int \frac{S_{D}(\omega) \sigma_{A}(\omega)}{\omega^{4}} d \omega,
$$

where $\mathrm{c}$ is the speed of light in vacuum, $\mathrm{n}$ is the refractive index of the organic medium, $\kappa^{2}$ is an orientation factor, $\eta_{D}$ is the donor PL quantum efficiency, $S_{D}$ is the emission spectrum of FIrpic (normalized to integrate over frequency to unity), and $\sigma_{\mathrm{A}}$ is the QD absorption cross-section. Using $\mathrm{n}=1.7, \kappa^{2}=2 / 3$, and $\eta_{\mathrm{D}}=80 \%$, we obtain $\mathrm{R}_{\mathrm{F}}=5.2 \mathrm{~nm}$. The total rate of energy transfer from a single FIrpic molecule to a QD layer is then obtained by integrating over all of the particles in a closely-packed QD monolayer: ${ }^{[72]}$

$$
K_{D-A L}=\frac{R_{F}^{6}}{\tau} \int_{r=0}^{r=\infty} \frac{2 \pi \rho_{A} r d r}{\left(r^{2}+D^{2}\right)^{3}}=\frac{R_{F}^{6}}{2 a^{2} D^{4} \tau}=\frac{1}{\tau}\left(\frac{D_{F}}{D}\right)^{4},
$$

where a is the QD radius, $\tau$ is the donor exciton lifetime, $\mathrm{D}$ is the distance from a donor to a plane of QD acceptors, $\rho_{A}$ is the QD surface density, and $\mathrm{D}_{\mathrm{F}}$ is the Förster distance for energy transfer from a single donor to a plane of acceptors. For energy transfer from a FIrpic molecule to a monolayer of green CdSe/ZnS QDs (a=2.1 nm), $\mathrm{D}_{\mathrm{F}} \sim 7 \mathrm{~nm}$ is obtained. This value is in a 
good agreement with the experimental result which shows that $D_{F}$ should be on the order of 8-10 $\mathrm{nm}$. The small discrepancy may be due to the fact that the QD layer is slightly thicker than one monolayer. In addition, exciton diffusion may supply additional excitons to the region within $\mathrm{D}_{\mathrm{F}}$ of the QD layer, leading to a larger effective transfer distance. However, given that most triplet excitons are localized on discrete FIrpic molecules, the effective diffusion length should be relatively small.

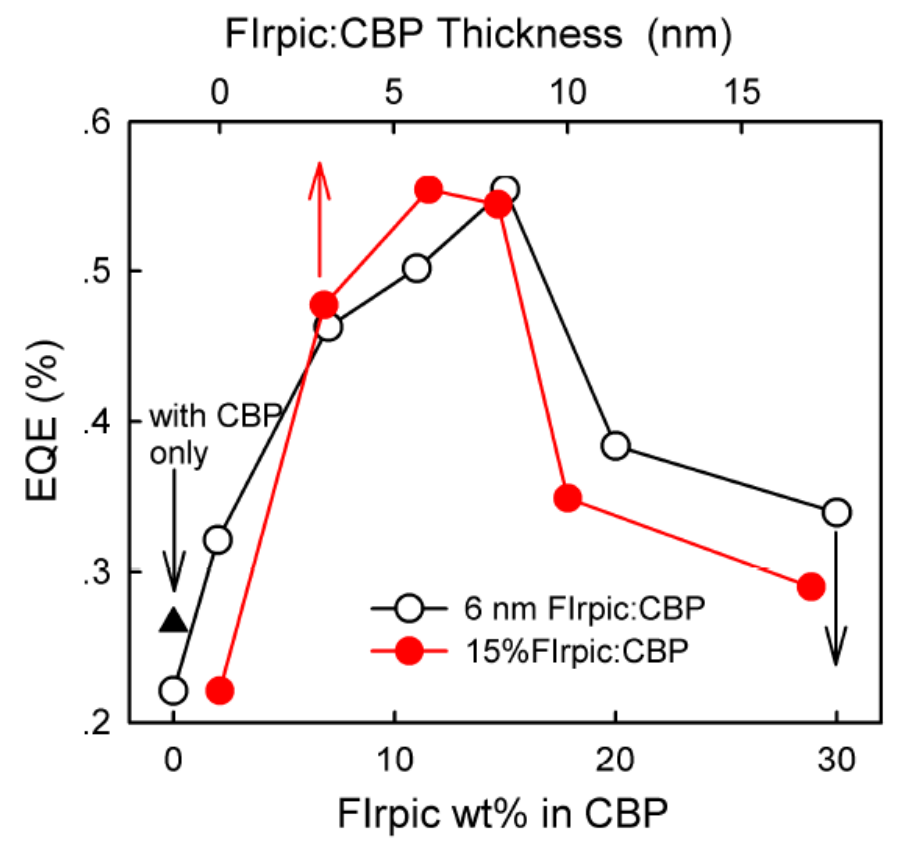

\section{Figure 3.10 EQE of green QD-LEDs}

EQE of green QD-LEDs measured at $80 \mathrm{~mA} / \mathrm{cm}^{2}$, as a function of the FIrpic doping concentration in a $6 \mathrm{~nm}$ CBP layer (black line) and the thickness of a CBP layer doped with 15\% FIrpic (red line).

Figure 3.10 shows the EQE of QD-LEDs measured at $80 \mathrm{~mA} / \mathrm{cm}^{2}$, as a function of the concentration of FIrpic doped in a $6 \mathrm{~nm}$ CBP layer. All the QD-LEDs exhibit EL enhancement over the control device, whose performance is comparable to similar devices reported in the literature. ${ }^{[16]}$ The EQE increases and then decreases with increasing FIrpic doping concentration. At low doping levels, a small amount of phosphorescent donors limits the rates of exciton harvesting and subsequent energy transfer. When the FIrpic concentration is raised, concentration quenching, which originates from long-range Förster energy transfer between FIrpic 
molecules, ${ }^{[78]}$ is expected to play a greater role and compete with exciton energy transfer to QDs. The EQE has the peak value of $0.55 \%$ at the FIrpic concentration of $15 \%$, a 2.5 -fold increase compared to that of the control QD-LED. Also plotted in Figure 3.10 is the EQE of a QD-LED with a neat CBP layer. The EQE is only increased by $20 \%$, confirming that, as compared to phosphorescent FIrpic, florescent CBP is a less efficient exciton energy donor. Figure 3.10 also shows the dependence of the EQE of QD-LEDs on the thickness of the CBP layer with a fixed FIrpic doping of 15\%. The highest EQE is obtained for QD-LEDs with a 6-8 nm FIrpic:CBP layer. As the donor layer grows thicker, the EL from the QDs decreases. This is because, as the light emitting zone broadens, an increasing portion of excitons are generated on FIrpic molecules outside the energy transfer region and undergo radiative decay.

The EQE of the QD-LEDs is still relatively low even with an optimized FIrpic donor layer. The performance is believed to be limited by several material and structural factors. First, imbalanced charge injection into the QDs leads to accumulation of electrons in the QDs and consequent nonradiative Auger recombination. ${ }^{[55]}$ Second, surface defects of the CdSe/ZnS QDs, which manifest themselves as long-wavelength tails in the EL spectra shown in Figure 3.7 and Figure 3.8, may have a significant negative impact on the energy transfer efficiency. Third, in the CBP-FIrpic host-guest system, triplet energy transfer is an endothermic process, limiting the exciton harvesting rate. Replacing CBP with a host having a higher triplet energy, such as N, N'dicarbazolyl-3,5-benzene (mCP), will enable exothermic energy transfer to FIrpic, ${ }^{[79]}$ and thus enhance the subsequent energy transfer to QDs. Finally, the QD layer and QD-LED structure used in this study are not optimized to realize the maximal exciton generation and energy transfer. The QD layer acts as a hole blocking layer, limiting the number of injected holes and thus the number of excitons formed in the CBP layer. An ideal hybrid structure would consist of a random mixture of QDs and phosphorescent donors with an appropriate mole ratio, embedded in a host layer. 


\subsection{Conclusions}

In summary, CdSe/ZnS QD-LEDs were fabricated by integrating the QD emission layer into a hybrid ITO/PEDOT:PSS/poly-TPD/QD/BCP/Alq ${ }_{3} / \mathrm{LiF} / \mathrm{Al}$ structure. The QD layer thickness was optimized by spin-coating QD solutions of different concentrations in the range of 1-10 $\mathrm{mg} / \mathrm{ml}$. The best concentration of QD solutions was determined to be $3 \mathrm{mg} / \mathrm{ml}$, corresponding to 1.6 MLs of green CdSe/ZnS QDs. Pure QD EL was achieved with a moderate turn-on voltage of $\sim 4 \mathrm{~V}$ from $3 \mathrm{mg} / \mathrm{ml}$ QD solution. The optimized QD-LED was used as the baseline device in our further work on performance enhancement. We demonstrated that the EL of green QD-LEDs can be significantly enhanced by using blue phosphorescent dyes as efficient exciton harvesters and energy donors. Precise control of the concentration of the FIrpic donors doped in a CBP host and their distance from the QD layer led to complete exciton energy transfer and EL enhancement by a factor of 2.5. The Förster distance between FIrpic molecules and green CdSe/ZnS QDs was determined to be $8-10 \mathrm{~nm}$, in a good agreement with the value calculated using the Förster model. Our study shows that the integration of QDs with phosphorescent organic dyes can overcome the difficulty of charge injection into QDs with bulky organic ligands, and provide an effective route to improve the EQE of QD-based hybrid LEDs. 


\section{Chapter 4 Synthesis and Characterization of CdSe QDs with}

\section{Inorganic Ligands}

Surface ligands play an important role in the synthesis of colloidal nanomaterials. ${ }^{[15]}$ They control the nucleation and growth of QDs, and the chemical and colloidal stability of QD solutions. The surface ligands are also extremely important for assembling individual particles into a QD solid. They contribute to the interparticle interactions and determine the spacing between neighboring QDs in short- and long-range ordered QD assemblies. Since the interparticle spacings have an extremely strong impact on the electronic properties of QD solids. The importance of surface ligands can hardly be overemphasized. Because of high surface-tovolume ratio inherent to the nanoscale objects, the surface has a strong influence on the physical properties of QDs such as photophysics, ${ }^{[80]}$ and charge transport. ${ }^{[81-82]}$ Proper surface chemistry is considered a key to make good QD-based photodetectors, ${ }^{[83]}$ solar cells, ${ }^{[12]}$ transistors, ${ }^{[4]}$ or LEDs. ${ }^{[84]}$

The most commonly used ligands for the passivation of QDs are based on organic hydrocarbon molecules with anchoring end groups. Such molecular structure offers the highest chemical flexibility. However, it has a number of serious drawbacks. Most organic ligands act as bulky insulating barriers between QDs, hindering charge transport. The complete removal of organic surface ligands has proven to be very difficult; it often generates multiple surface dangling bonds and midgap charge-trapping states. ${ }^{[80]}$ Annealing of QD samples can partially remove the organic ligands, but it often leads to sintering of QDs. ${ }^{[85]}$ Moreover, high temperature annealing typically leaves behind undesirable carbonaceous species due to partial ligand pyrolysis. ${ }^{[86]}$ Simple exchange of original bulky ligands by smaller molecules is a widely used 
approach for improving charge transport. Mild chemical treatments with dilute hydrazine solutions, ${ }^{[4]}$ phenylenediamine, ${ }^{[81]}$ and other crosslinking molecules enabled considerable progress in the preparation of conductive QD solids. However, these small molecules are not stable against oxidation and volatility, leading to instabilities of the electronic properties. Furthermore, conventional semiconductor technologies always prefer pure, all-inorganic, and highly crystalline materials, which stimulated the development of functional all-inorganic ligand coatings ${ }^{[46-47]}$.

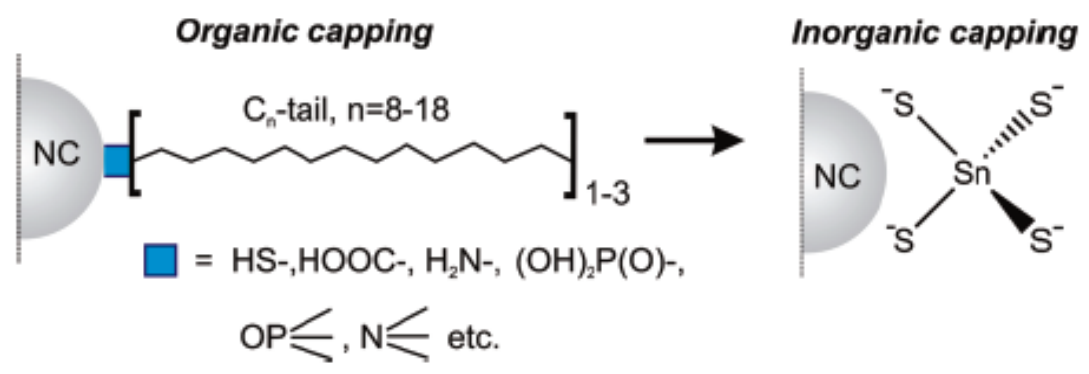

Figure 4.1 Schematics of the ligand exchange process

Schematics of the ligand exchange process used for preparation of all-inorganic QDs ${ }^{[47]}$

Ideal surface ligands for QDs should provide colloidal stabilization, electronic passivation, and facile interparticle electronic communication. Recently, Kovalenko and co-workers ${ }^{[46-48]}$ developed a generalized approach to replace the original organic ligands with inorganic molecular metal chalcogenide complexes (MCCs), which enabled strong electronic coupling in various QD solids due to their small size and semiconducting property. In field-effect transistors with a CdSe QD channel, replacing organic ligands with MCCs led to an increase in conductivity by many orders of magnitude ${ }^{[46]}$ However, the optical properties of the novel MCC-capped QDs have not yet systematically studied so far. In this chapter, we develop a similar process for exchanging the ODA ligands of CdSe core and core/shell QDs with inorganic $\mathrm{SnS}_{4}$ ligands in hydrazine solvent, and further optimize it in ammonia hydroxide solvent. Furthermore, the applicability of $\mathrm{SnS}_{4}$-capped CdSe QDs for optoelectronic applications is evaluated through detailed optical characterization of QDs in solids before and after the ligand exchange. 


\subsection{Ligand Exchange in Hydrazine Solvent}

To perform the ligand exchange in hydrazine solvent, the CdSe core and CdSe/ZnS core/shell QDs with organic ligands were first synthesized using the method described in Chapter 2. CdSe core QDs are initially capped with ODA/TOPO ligands, and CdSe/ZnS core/shell QDs are mainly capped with ODA ligands. And then MCC ligands of $\left(\mathrm{N}_{2} \mathrm{H}_{5}\right)_{4} \mathrm{Sn}_{2} \mathrm{~S}_{6}$ are synthesized in hydrazine $\left(\mathrm{N}_{2} \mathrm{H}_{4}\right)$ solvent, following an approach recently developed by Talapin and coworkers. ${ }^{[46]}\left(\mathrm{N}_{2} \mathrm{H}_{5}\right)_{4} \mathrm{Sn}_{2} \mathrm{~S}_{6}$ was prepared by dissolving elemental tin $(118.7 \mathrm{mg}, 1 \mathrm{mmol})$ in a mixture of $3 \mathrm{~mL} \mathrm{~S}-\mathrm{N}_{2} \mathrm{H}_{4}(1 \mathrm{M})$ solution and $1 \mathrm{~mL} \mathrm{~N}_{2} \mathrm{H}_{4}$ upon stirring at $80-90{ }^{\circ} \mathrm{C}$ until a light yellow solution was formed. Finally, ligand exchange of organically capped QDs with MCC ligands are conducted by interface reactions between hexane solvent and hydrazine solvent, as schematically shown in Figure 4.2. The ligand-exchanged QDs were prepared in a nitrogen-filled glovebox. $6 \mathrm{~mL} \mathrm{~N} \mathrm{~N}_{2} \mathrm{H}_{4}$ was mixed with $\left(\mathrm{N}_{2} \mathrm{H}_{5}\right)_{4} \mathrm{Sn}_{2} \mathrm{~S}_{6}(25 \mu \mathrm{L}, \sim 0.5 \mathrm{M})$. Hexane $(4 \mathrm{~mL})$ and ODAcapped QDs (25 mg) were loaded onto the top of the hydrazine phase. The mixture was vigorously stirred overnight until the phase transfer of QDs was completed. The hydrazine phase was washed with hexane 3 times, filtered, and mixed with acetonitrile $(1: 1 \mathrm{v} / \mathrm{v})$ to precipitate QDs. After centrifuging, QDs were redissolved in $5 \mathrm{~mL}$ of hydrazine solvent.
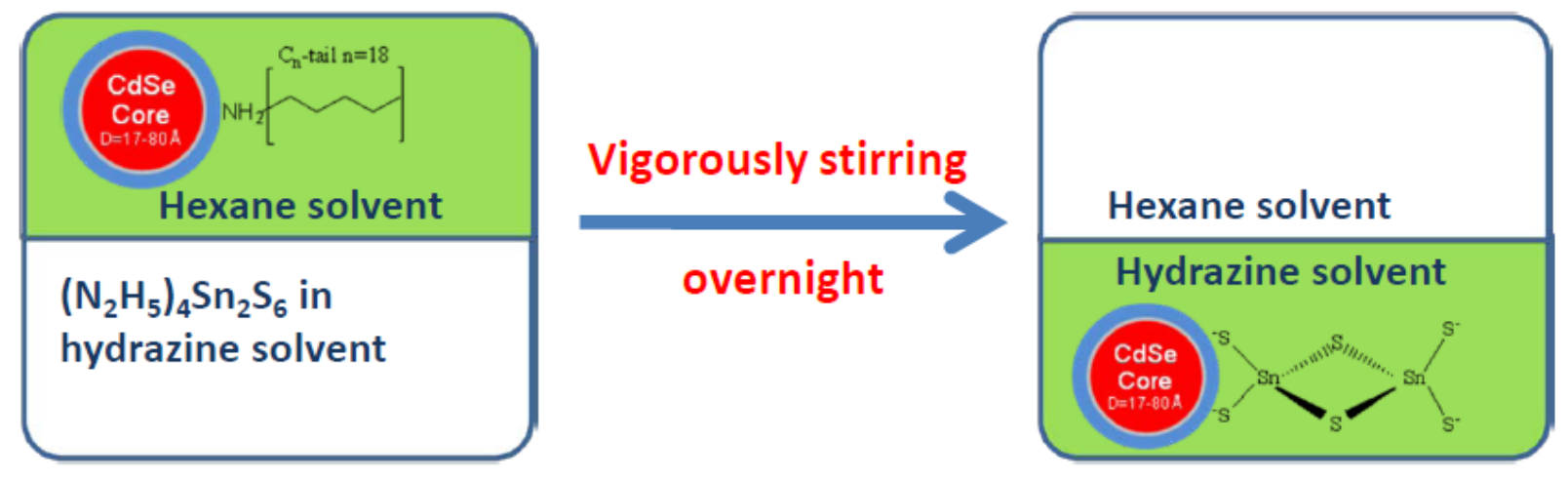

Figure 4.2 Schematics of organic-to-MCC ligands exchange in hydrazine solvent

The organic ligands for passivating both CdSe core QDs and CdSe/ZnS core/shell QDs 
were successfully replaced by $\mathrm{Sn}_{2} \mathrm{~S}_{6}{ }^{4-}$ ligands. The degree of ligand exchange was evaluated by Fourier-transform infrared (FTIR) spectroscopy. ${ }^{[46]}$ For CdSe-based QDs, after the exchange of the original organic ligands with $\mathrm{Sn}_{2} \mathrm{~S}_{6}{ }^{4-}$ ligands in hydrazine, the strong absorption lines arising from characteristic C-H and N-H stretching vibrations at 2800-3500 $\mathrm{cm}^{-1}$ disappeared (Figure 4.3). This confirms the success of ligand exchange from ODA ligands to $\mathrm{Sn}_{2} \mathrm{~S}_{6}{ }^{4-}$ ligands. ${ }^{[46]}$

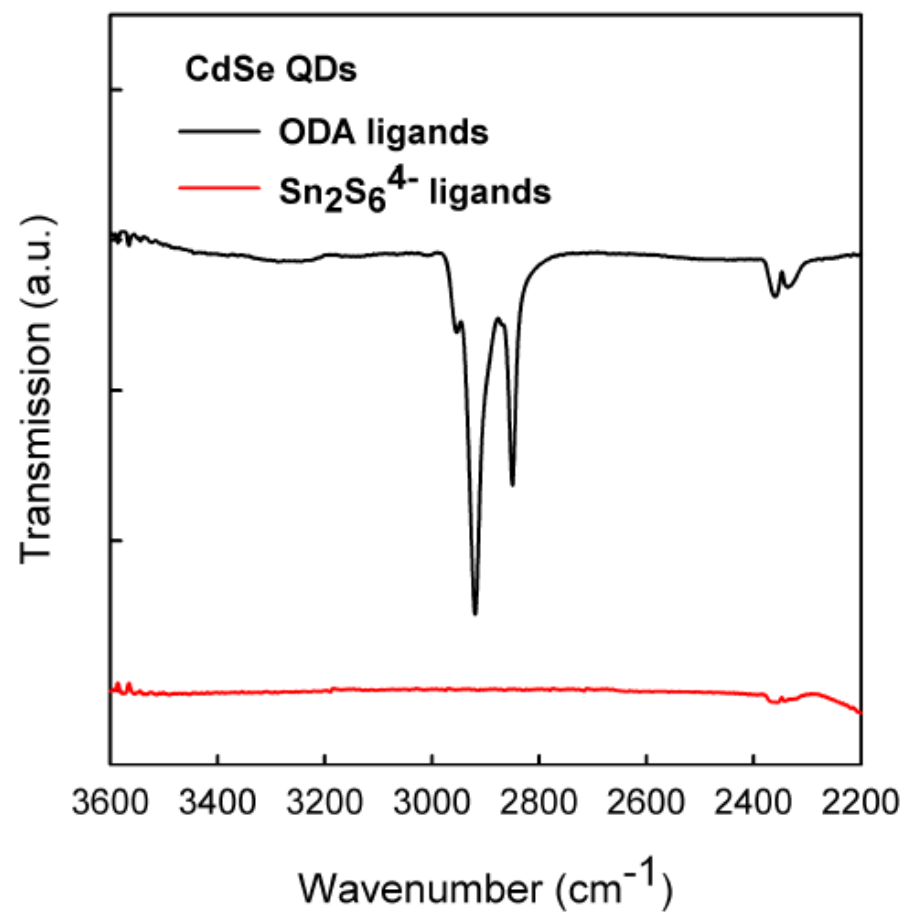

Figure 4.3 FTIR spectra of CdSe QDs capped with original organic ligands and after ligand exchange with $\left(\mathrm{N}_{2} \mathrm{H}_{5}\right)_{4} \mathrm{Sn}_{2} \mathrm{~S}_{6}$. The spectra are normalized to the amount of absorbing materials and are vertically shifted for clarity

The optical characterization results of QDs in solution are shown in Figure 4.4 and Figure 4.5. For CdSe core QDs, the absorption spectrum stays the same after ligand exchange (Figure 4.4 a). This means strong light absorption of QDs is retained after ligand exchange. In contrast, the PL spectra showed a broadening and a pronounced $20 \mathrm{~nm}$ red shift after ligand exchange (Figure $4.4 \mathrm{~b}$ ), implying partial leakage of the wave function into neighboring QDs that relaxes the quantum confinement. The QY of CdSe core QDs was found to be reduced from 10\% to 
0.01\% after ligand exchange.
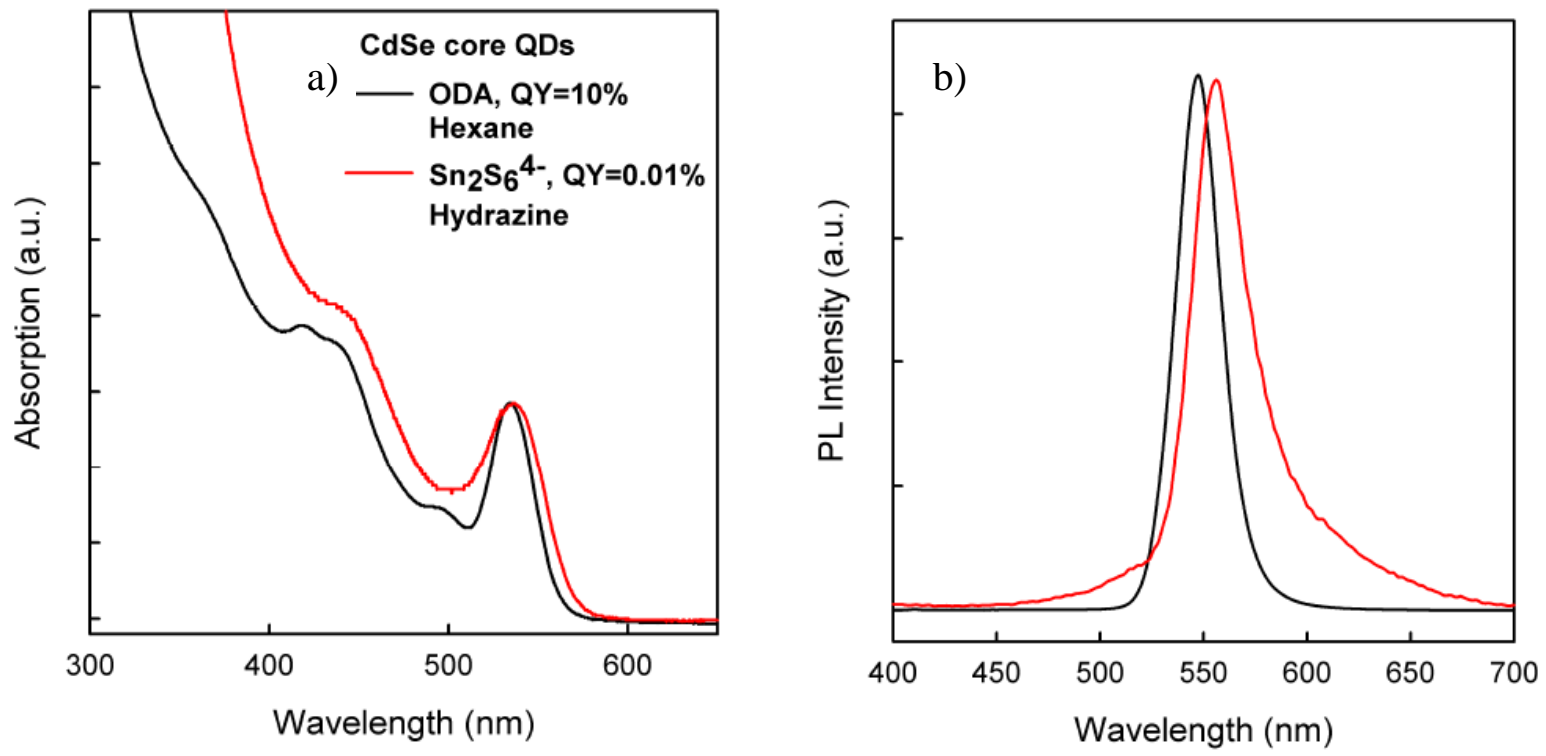

Figure 4.4 Normalized (a) absorption and (b) PL spectra of CdSe core QDs with organic ODA ligands in hexane solvent or with inorganic $\mathrm{Sn}_{2} \mathrm{~S}_{6}{ }^{4}$ ligands in hydrazine solvent

For CdSe/ZnS core/shell QDs, the absorption and PL spectra before and after ligand exchange are very similar to each other, indicative of strong localization of electron and hole wave functions in individual QDs enabled by the nanoscale ZnS shell (Figure 4.5). The peak red shift of the PL spectrum is much smaller compared to that in the case of core QDs. Unfortunately, the PL QY of the CdSe/ZnS core/shell QDs was also significantly reduced after the exchange in hydrazine, from $50 \%$ to $3 \%$. 

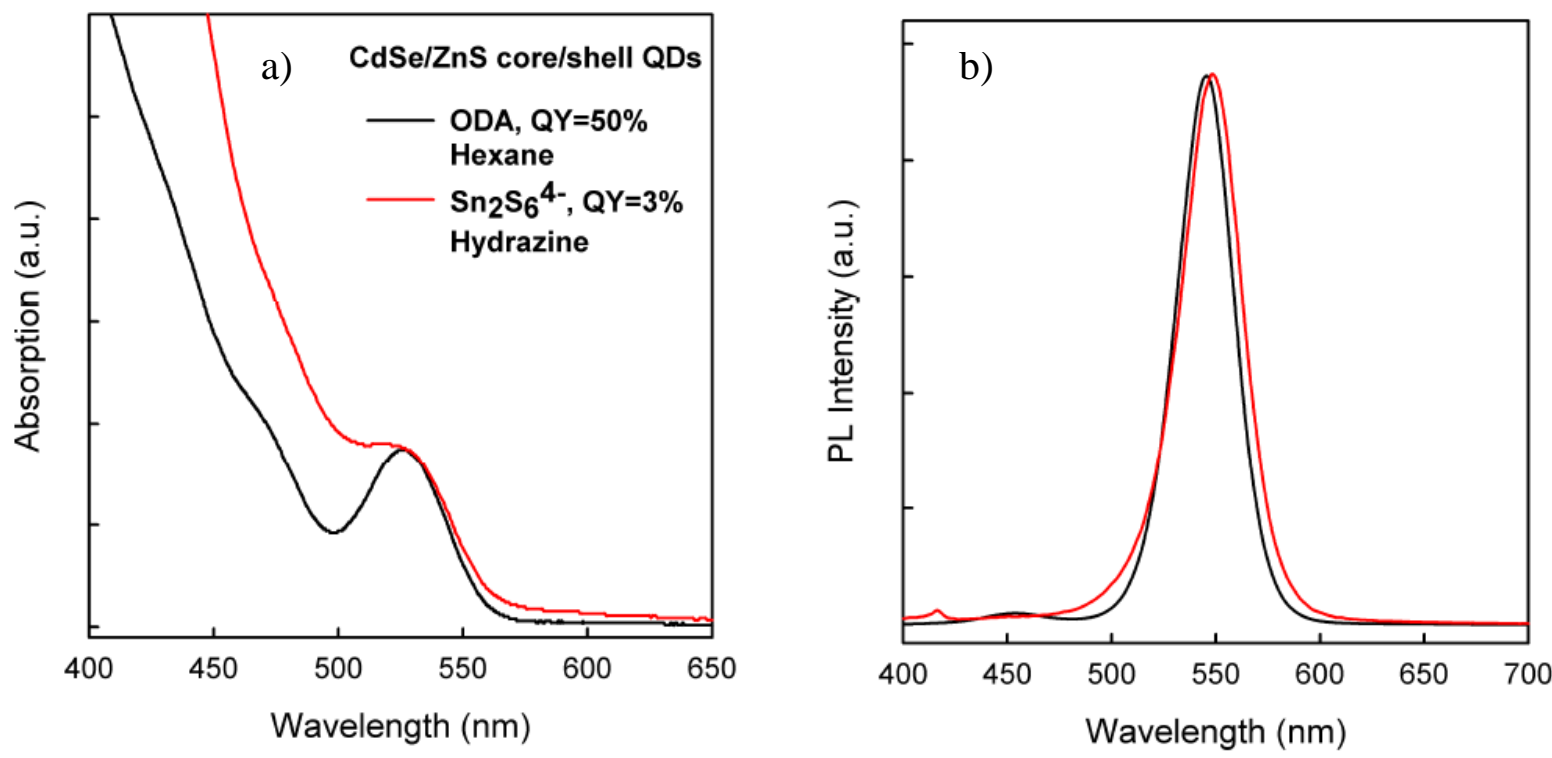

Figure 4.5 Normalized (a) absorption and (b) PL spectra of CdSe/ZnS core/shell QDs with organic ODA ligands in hexane solvent or with inorganic $\mathrm{Sn}_{2} \mathrm{~S}_{6}{ }^{4-}$ ligands in hydrazine solvent

\subsection{Ligand Exchange in Ammonia Hydroxide Solvent}

Although high colloidal stability can be achieved with the new MCC ligands in hydrazine mainly due to the negative charge of the ligands, the superior optical properties of QDs fail to retain. The result suggests that hydrazine may be very harsh to attack the QD surfaces. More

effective ligand exchange is thus expected in a more benign aqueous solvent. ${ }^{[47]}$ Here, we conduct similar ligand exchange in a $\mathrm{NH}_{4} \mathrm{OH}$ solution, which is able to better preserve the optical properties of CdSe QDs. The process is described in detail below. 


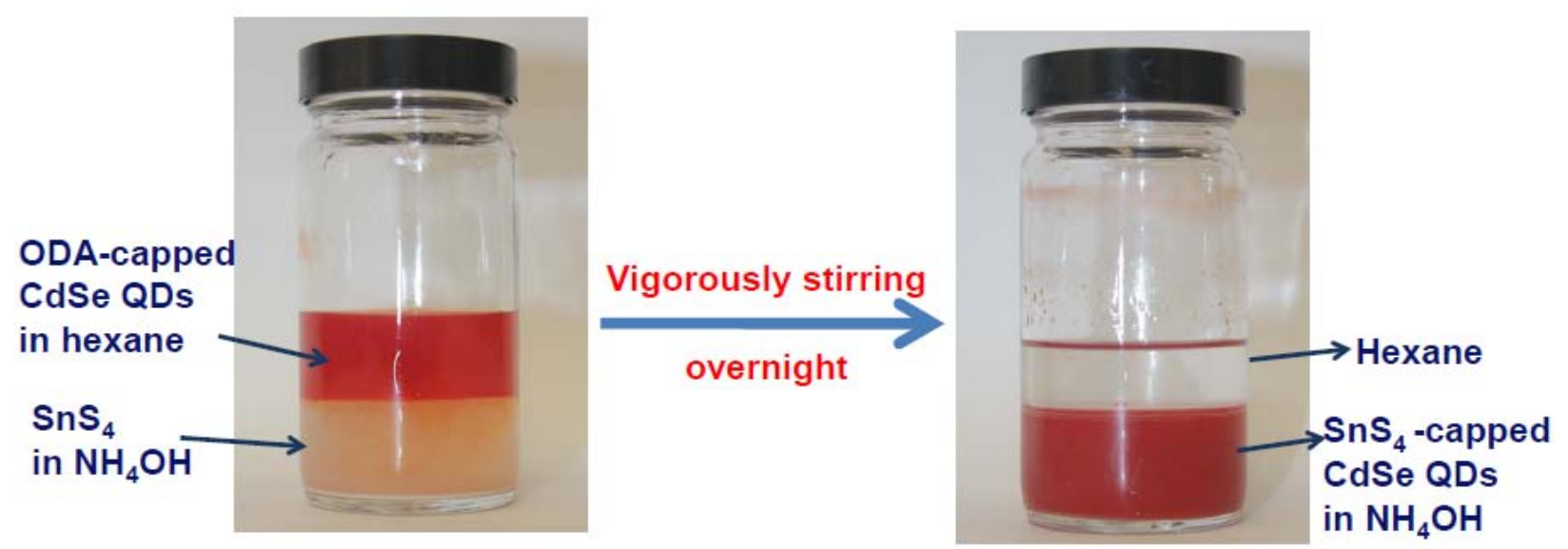

Figure 4.6 Schematics of organic-to-MCC ligand exchange in ammonia hydroxide solvent

Yellow colloidal CdSe core QDs, $3.2 \mathrm{~nm}$ in diameter, were synthesized using the conventional hot-injection method. To grow CdSe/CdS/ZnS core/multishell QDs, 2 monolayer (ML) CdS shell and $3 \mathrm{ML} \mathrm{ZnS} \mathrm{shell} \mathrm{were} \mathrm{coated} \mathrm{on} \mathrm{the} \mathrm{CdSe} \mathrm{core} \mathrm{using} \mathrm{SILAR} \mathrm{method.} \mathrm{The}$ QDs were subjected to a multistep precipitation and redissolvation process of purification to remove side products and unreacted precursors. The as-synthesized QDs were capped with bulky organic ODA ligands. Metal chalcogenide species $\mathrm{Na}_{4} \mathrm{SnS}_{4}$ was prepared by dissolving $\mathrm{SnS}_{2}$ (1mmol, $183 \mathrm{mg})$ in aqueous $\mathrm{Na}_{2} \mathrm{~S}$ (2mmol) at high $\mathrm{pH}\left(\mathrm{pH}=11-12\right.$ for $\left.\mathrm{Na}_{4} \mathrm{SnS}_{4}\right)$. $\mathrm{Na}_{4} \mathrm{SnS}_{4} \cdot \mathrm{xH}_{2} \mathrm{O}(\mathrm{x} \leq 14)$ was precipitated by the addition of acetone, followed by centrifuging and drying. Most of crystalline water can be removed by moderate heating at $100-200{ }^{\circ} \mathrm{C}$. The ligand-exchanged QDs were prepared in a nitrogen-filled glovebox, as seen from Figure 4.6. Aqueous $\mathrm{NH}_{4} \mathrm{OH}\left(32 \mathrm{~mL}, 28-30 \%\right.$ of $\mathrm{NH}_{3}$ ) were mixed with aqueous $\mathrm{Na}_{4} \mathrm{SnS}_{4}(4 \mathrm{ml}, \sim 0.1 \mathrm{M})$. Hexane (24 mL) and QDs in hexane (4 mL, $25 \mathrm{mg} / \mathrm{mL}$ ) were then loaded onto the top of the aqueous phase. The solution was stirred for 4 hours until the phase transfer of QDs was completed. During this step, the organic ligands were stripped off from the QD surfaces, leaving behind electron-deficient metal atoms with a strong affinity toward bonding with $\mathrm{S}$ in $\mathrm{SnS}_{4}{ }^{4-}$ ions that are neutralized mainly by $\mathrm{NH}_{4}{ }^{+}$counterions. ${ }^{[47]}$ The negatively charged ligands prevent aggregation of the QDs, resulting in a stable colloidal solution of QDs. The $\mathrm{SnS}_{4}$-capped QDs in 
aqueous $\mathrm{NH}_{4} \mathrm{OH}$ phase was washed with hexane 3 times, filtered, precipitated by adding acetonitrile (1:1 v/v), centrifuged, and re-dissolved in $5 \mathrm{~mL} \mathrm{NH} \mathrm{NH}_{4} \mathrm{OH}$ solvent.
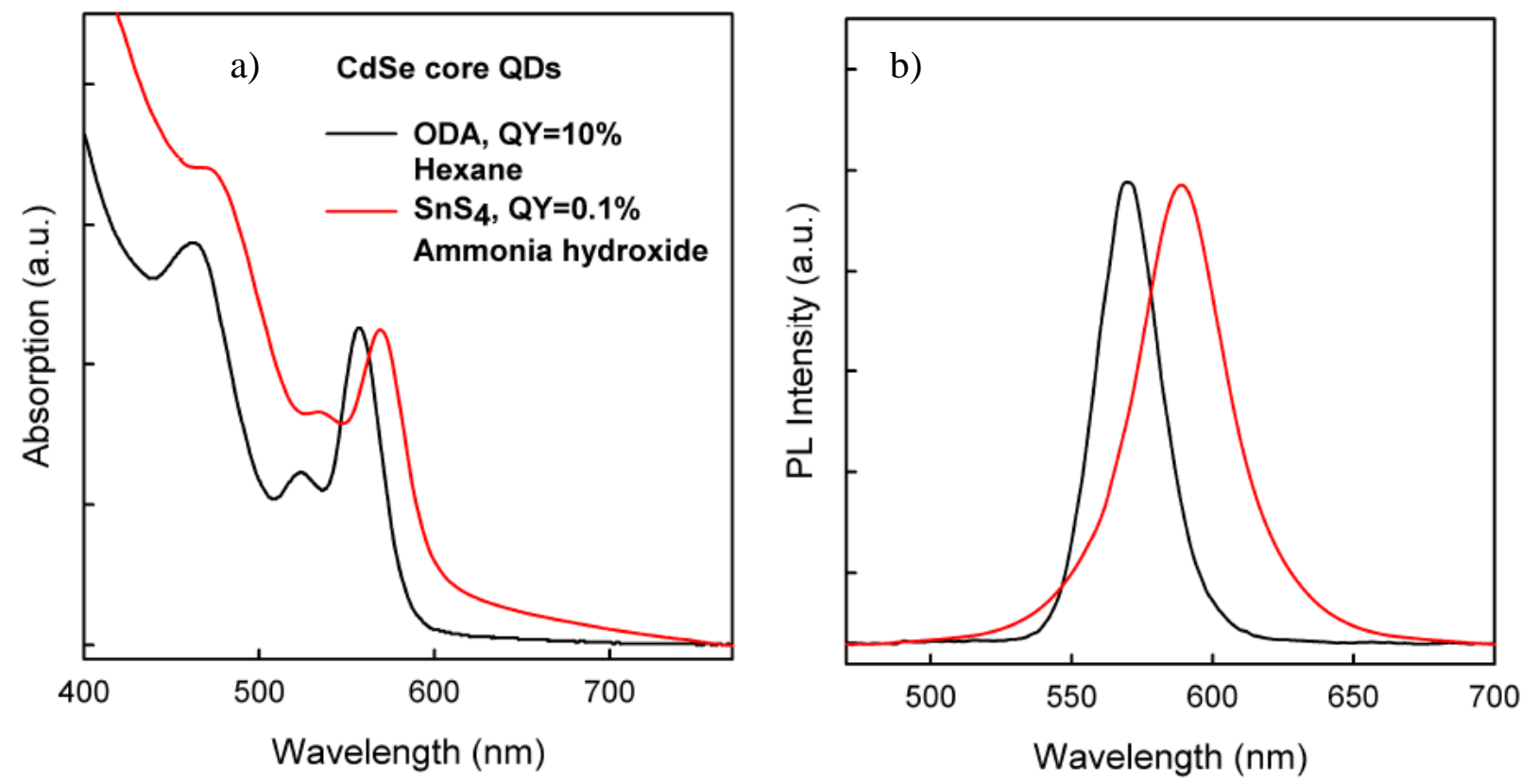

Figure 4.7 Normalized (a) absorption and (b) PL spectra of CdSe core QDs with organic ODA ligands in hexane solvent or with inorganic $\mathrm{SnS}_{4}$ ligands in ammonia hydroxide solvent

The normalized absorption and PL spectra of CdSe core QD solutions before and after the ligand exchange are shown in Figure 4.7. The absorption spectra of the QDs with organic ligands in hexane solvent features size-dependent excitonic absorption peaks. After the ligand exchange, the spectra exhibit a redshift and significant broadening. The first excitonic peak shifts from $557 \mathrm{~nm}$ to $569 \mathrm{~nm}$. The change can be explained by partial delocalization of electron and hole wave functions due to a lower energy barrier at the $\mathrm{QD} / \mathrm{SnS}_{4}$ interface. A similar trend is seen from the PL data. Even in solution where inter-QD interaction is negligible, the ligand exchange leads to a pronounced redshift ( 18 nm) and spectral broadening of the PL spectrum. On the other hand, the fact that the PL and absorption peaks maintain their original shape suggests that the quantum confinement effects in the core QDs are largely retained. Unfortunately, after the ligand exchange, the core QDs could not retain their good luminescent properties. The PL QY of the QDs in solution dropped from $10 \%$ to below $0.1 \%$, suggesting 
that $\mathrm{SnS}_{4}$ ions cannot provide effective surface passivation as the original organic ligands. It may also result from the efficient diffusion of electrons and/or holes to the $\mathrm{SnS}_{4}$ ligands, leading to reduced radiative exciton decay inside QDs.
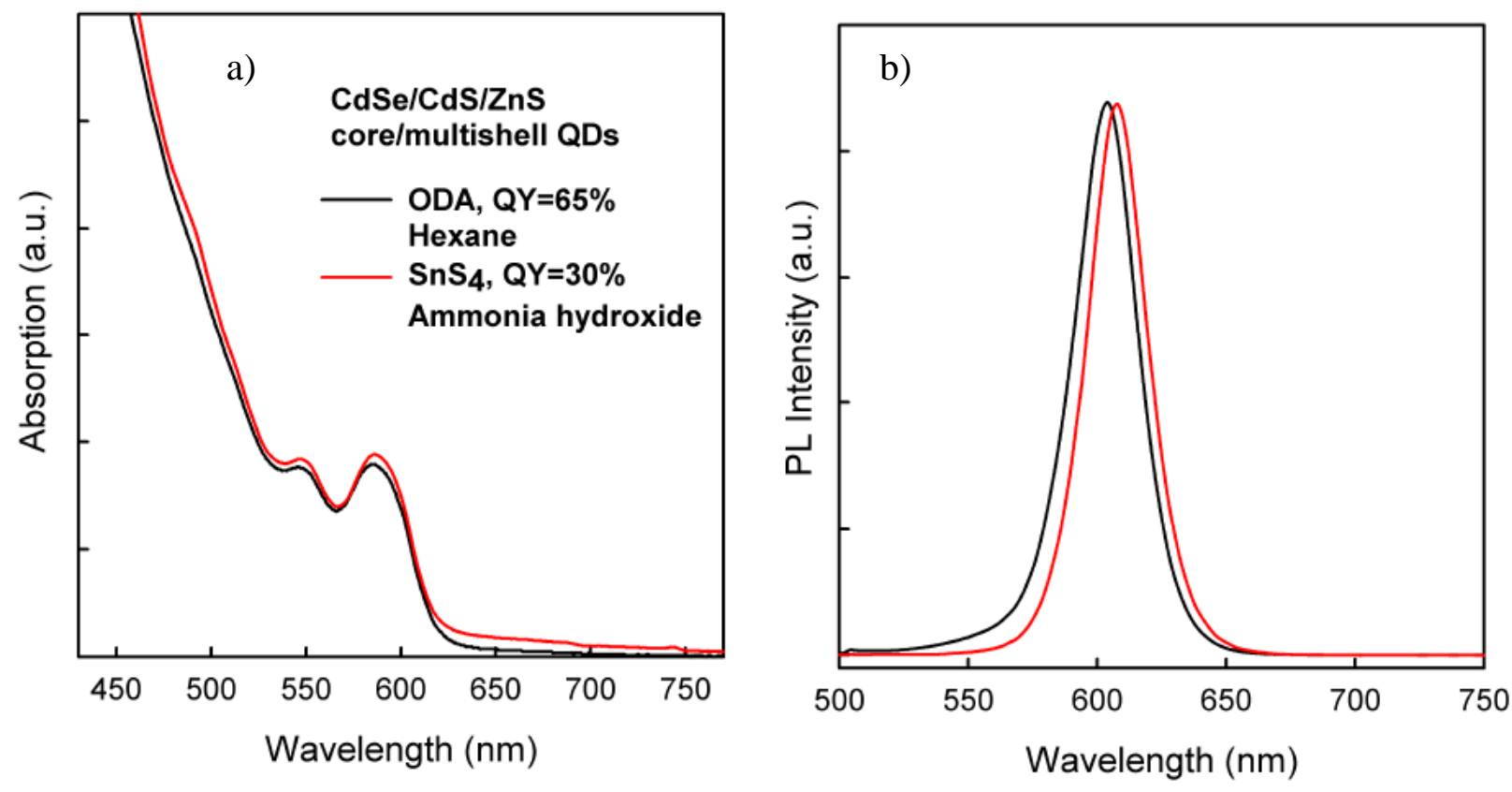

Figure 4.8 Normalized (a) absorption and (b) PL spectra of CdSe/CdS/ZnS core/multishell QDs with organic ODA ligands in hexane solvent or with inorganic $\mathrm{SnS}_{4}$ ligands in ammonia hydroxide solvent

It has been found that the CdS/ZnS multishell can significantly enhance exciton confinement and reduce the adverse effect caused by the ligand exchange, as shown in Figure 4.8. The CdSe/CdS/ZnS core/multishell QDs only exhibited a moderate reduction in the PL QY after the ligand exchange, from 65\% for QDs with ODA ligands in hexane to 30\% for QDs with $\mathrm{SnS}_{4}$ ligands in a $\mathrm{NH}_{4} \mathrm{OH}$ solution. After replacing the organic ligands with $\mathrm{SnS}_{4}$, the CdSe/CdS/ZnS QDs retain strong excitonic absorption and luminescence. The redshift and broadening of the PL and absorption peaks in solutions are significantly reduced compared to those of the CdSe core QDs. It is thus clear that a trade-off exists between thicker shells for better surface passivation as well as stronger quantum confinement and thinner shells for more facile electronic coupling. 


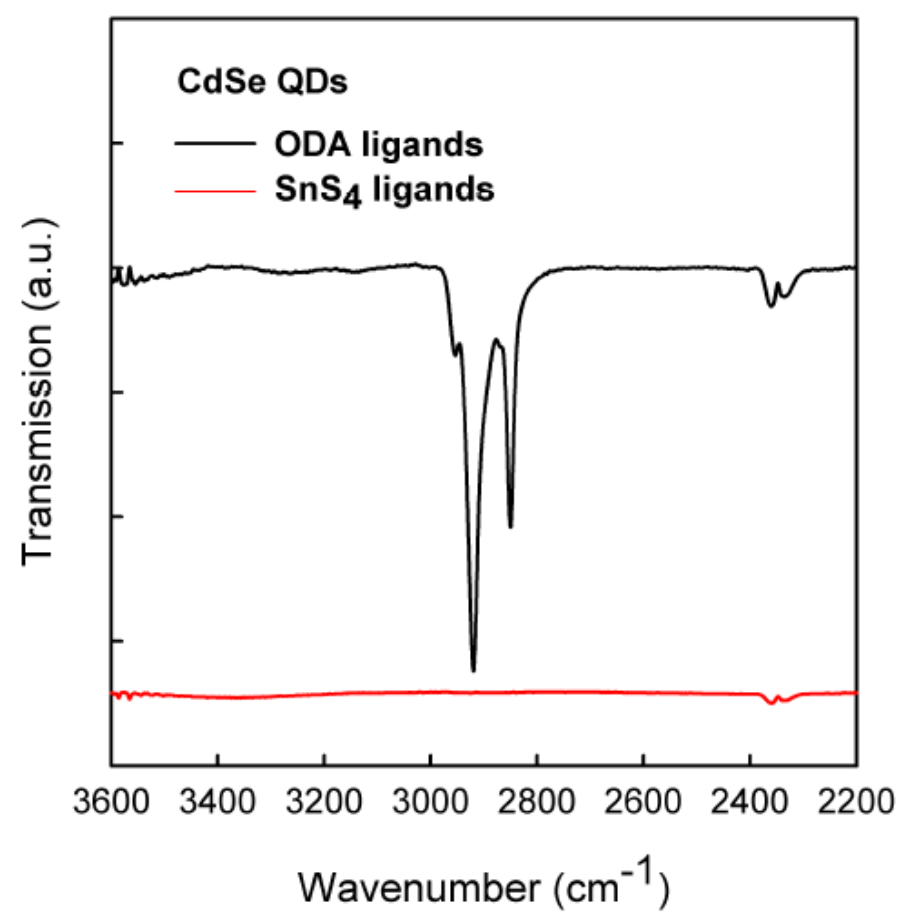

Figure 4.9 FTIR spectra of CdSe QDs capped with original organic ligands and after ligand exchange with $\mathrm{Na}_{4} \mathrm{SnS}_{4}$. The spectra are normalized to the amount of absorbing materials and are vertically shifted for clarity

The degree of ligand exchange was studied by FTIR spectroscopy. Figure 4.9 shows FTIR spectra obtained for CdSe-based QDs before and after the ligand. The strong absorption lines arising from characteristic C-H and N-H stretching vibrations at $2800-3500 \mathrm{~cm}^{-1}$ are removed after ligand exchange. The rate and completeness of the ligand exchange are similar as those we previously observed using $\left(\mathrm{N}_{2} \mathrm{H}_{5}\right)_{4} \mathrm{Sn}_{2} \mathrm{~S}_{6}$ ligand and hydrazine solvent (Figure 4.3), which confirms that the ligand exchange reaction is mainly driven by the competition between the original organic ODA ligands and the highly nucleophilic inorganic $\mathrm{SnS}_{4}$ ligands. ${ }^{[47]}$

\subsection{Optical Characterization of MCC-capped Quantum Dots Thin}

\section{Film}

The applicability of colloidal CdSe QDs with metal chalcogenide ligands for 
optoelectronic applications is evaluated here through detailed optical characterization of QDs thin film before and after the ligand exchange. For optical characterization, CdSe core or CdSe/CdS/ZnS core/multishell QD thin films $\sim 100 \mathrm{~nm}$ composed of disordered arrays of closely-packed QDs were prepared on clean glass by spin-coating from concentrated solutions of QDs with organic ODA ligands in hexane, or QDs with inorganic $\mathrm{SnS}_{4}$ ligands in $\mathrm{NH}_{4} \mathrm{OH}$. The QD films were then annealed in ambient $\mathrm{N}_{2}$ at $150-450{ }^{\circ} \mathrm{C}$ for $5 \mathrm{~min}$, and their absorption and PL spectra were measured using a Hitachi U-3900H double-beam UV-VIS spectrophotometer and a Hitachi F-7000 fluorescence spectrophotometer (365 nm excitation), respectively.
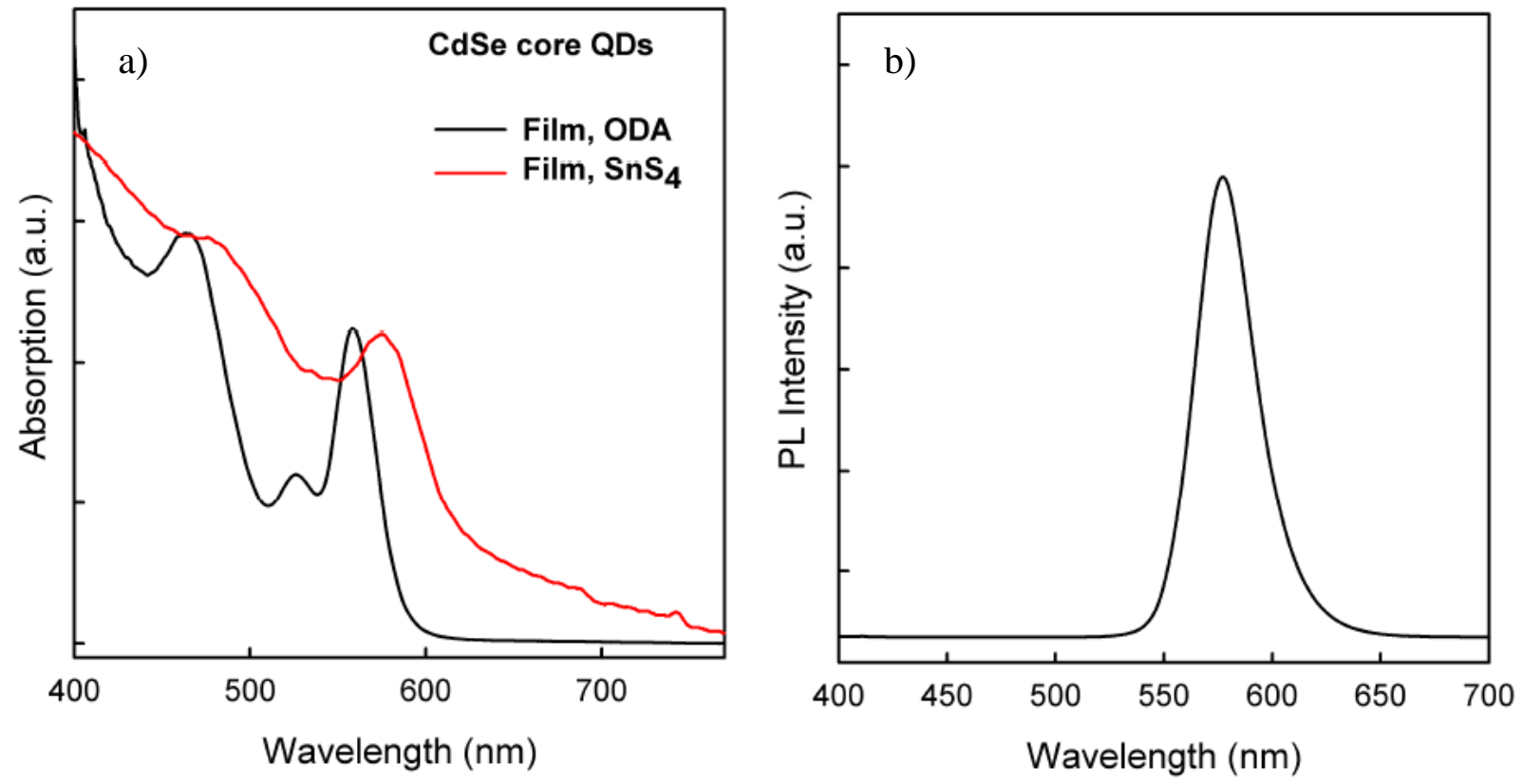

Figure 4.10 Normalized (a) absorption and (b) PL spectra of the films of CdSe core QDs with organic ODA or inorganic $\mathrm{SnS}_{4}$ ligands. The PL of QDs with $\mathrm{SnS}_{4}$ ligands is completely quenched at room temperature.

The normalized absorption and PL spectra of the CdSe core QD films before and after the ligand exchange are shown in Figure 4.10. After the ligand exchange, the absorption spectrum basically preserves its original shape featuring strong size-dependent excitonic absorption peaks, but exhibits a large redshift and significant broadening. The first excitonic peak shifts by $18 \mathrm{~nm}$, from $557 \mathrm{~nm}$ to $575 \mathrm{~nm}$. These changes indicate partial leakage of the electron and hole wave functions into the ligands and even neighboring QDs due to lower energy barriers at the 
$\mathrm{QD} / \mathrm{SnS}_{4}$ interface. ${ }^{[49]}$ It thus can be concluded that quantum confinement is largely retained in the QDs with inorganic $\mathrm{SnS}_{4}$ ligands, but partially reduced due to the inorganic capping layer. According to the Wentzel-Kramers-Brillouin (WKB) approximation, the dependence of the electron tunneling rate $(\Gamma)$ between adjacent QDs on the tunneling barrier heightE and the shortest edge-to-edge distance $\Delta \mathrm{x}$ can be expressed as: ${ }^{[87]}$

$$
\Gamma=\exp \left[-2\left(2 m^{*} \Delta E / \hbar^{2}\right)^{1 / 2} \Delta x\right]
$$

where $\mathrm{m}^{*}$ is the electron effective mass. The $\mathrm{SnS}_{4}$ ligands substantially reduced both $\Delta \mathrm{E}$ and $\Delta \mathrm{x}$ (from $>1.5 \mathrm{~nm}$ to $\sim 0.5 \mathrm{~nm}$ ), ${ }^{[15]}$ thus greatly enhanced the inter-QD electronic coupling. Therefore, we may expect that the ligand exchange will lead to more facile electronic communication and charge transport in QD solids.

The PL of the QDs with organic ligands shifted from $570 \mathrm{~nm}$ in a hexane solution to 577 $\mathrm{nm}$ in solid. Unfortunately, the ligand exchange was found to deteriorate the PL efficiency. The PL intensity dropped by a factor of $~ 100$ in solution, and was completely quenched in the QD thin film. The quenching may be attributed to a combination of: (i) the $\mathrm{SnS}_{4}$ ions cannot provide effective surface passivation as the original organic ligands, leaving dangling bonds on the QD surfaces as nonradiative recombination centers; (ii) leakage of carriers to the $\mathrm{SnS}_{4}$ capping layer leads to reduced overlap between the electron and hole wave functions and thus reduced radiative exciton decay inside the QDs; and (iii) excitons may diffuse fast through the allinorganic QD solid and more likely experience nonradiative decay at quenching sites. ${ }^{[45]}$ 

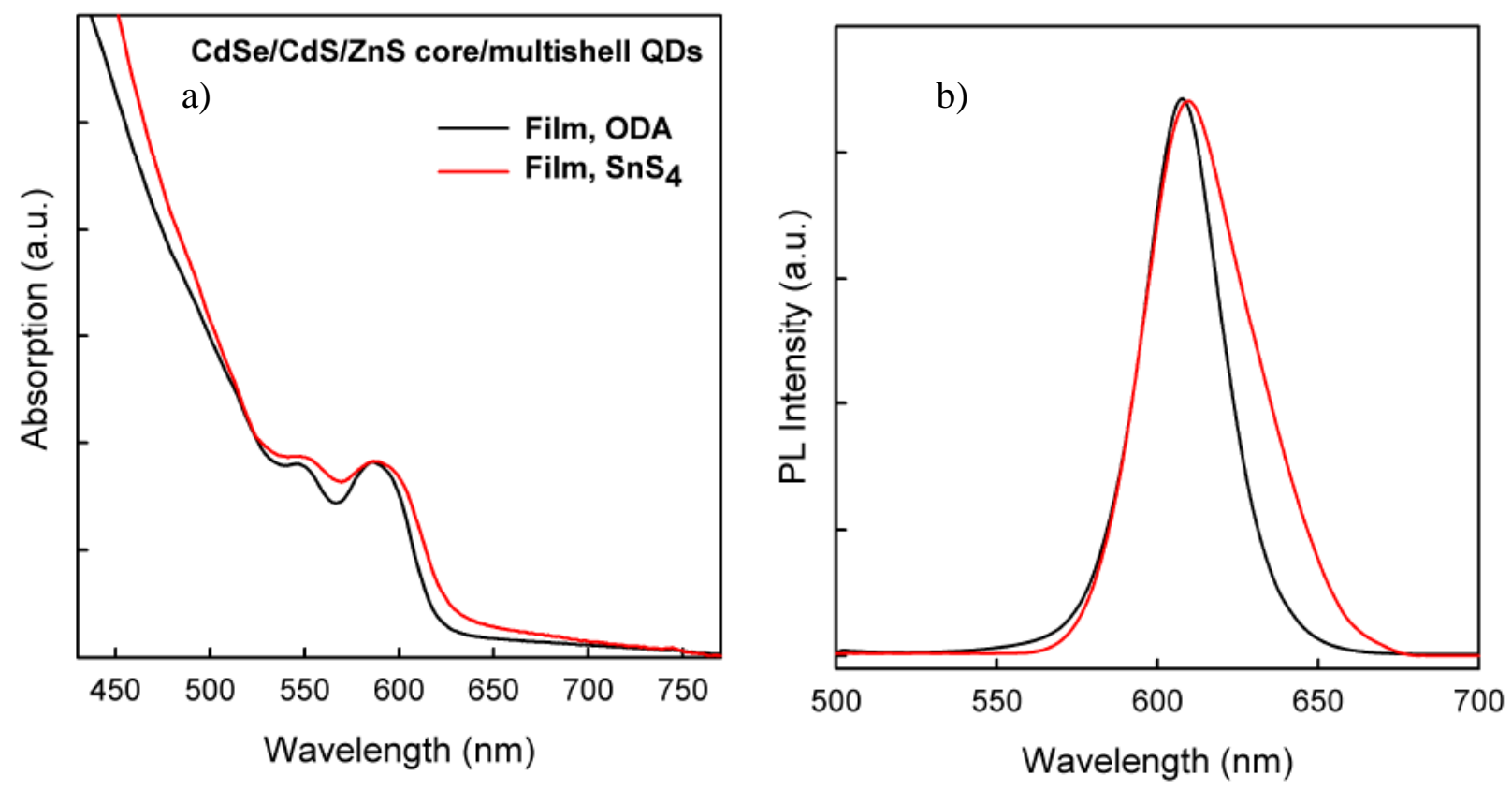

Figure 4.11 Normalized (a) absorption and (b) PL spectra of the films of CdSe/CdS/ZnS core/multishell QDs with organic ODA or inorganic $\mathrm{SnS}_{4}$ ligands

It has been found that the CdS/ZnS multishell can significantly enhance exciton confinement and reduce the adverse effect caused by the ligand exchange. In solutions, CdSe/CdS/ZnS core/multishell QDs only exhibited a moderate reduction in the PL QY by a factor of $\sim 2$ after the ligand exchange in a $\mathrm{NH}_{4} \mathrm{OH}$ solution. We were able to detect $\mathrm{PL}$ from the QD films with either organic or inorganic ligands, as seen in Figure 4.11. After replacing the organic ligands with $\mathrm{SnS}_{4}$, the $\mathrm{CdSe} / \mathrm{CdS} / \mathrm{ZnS}$ QDs retain strong excitonic absorption and luminescence. The redshift and broadening of the PL and absorption peaks in solids are significantly reduced compared to those of the CdSe core QDs. It is thus clear that a trade-off exists between thicker shells for better surface passivation as well as stronger quantum confinement and thinner shells for more facile electronic coupling. These findings indicate that, with small inorganic metal chalcogenide ligands, strong electronic coupling may be achieved between closely-packed QDs. This will lead to efficient QD-QD charge transport, which is critical for the operation of electronic and optoelectronic devices based on QD solids. ${ }^{[15]}$ 

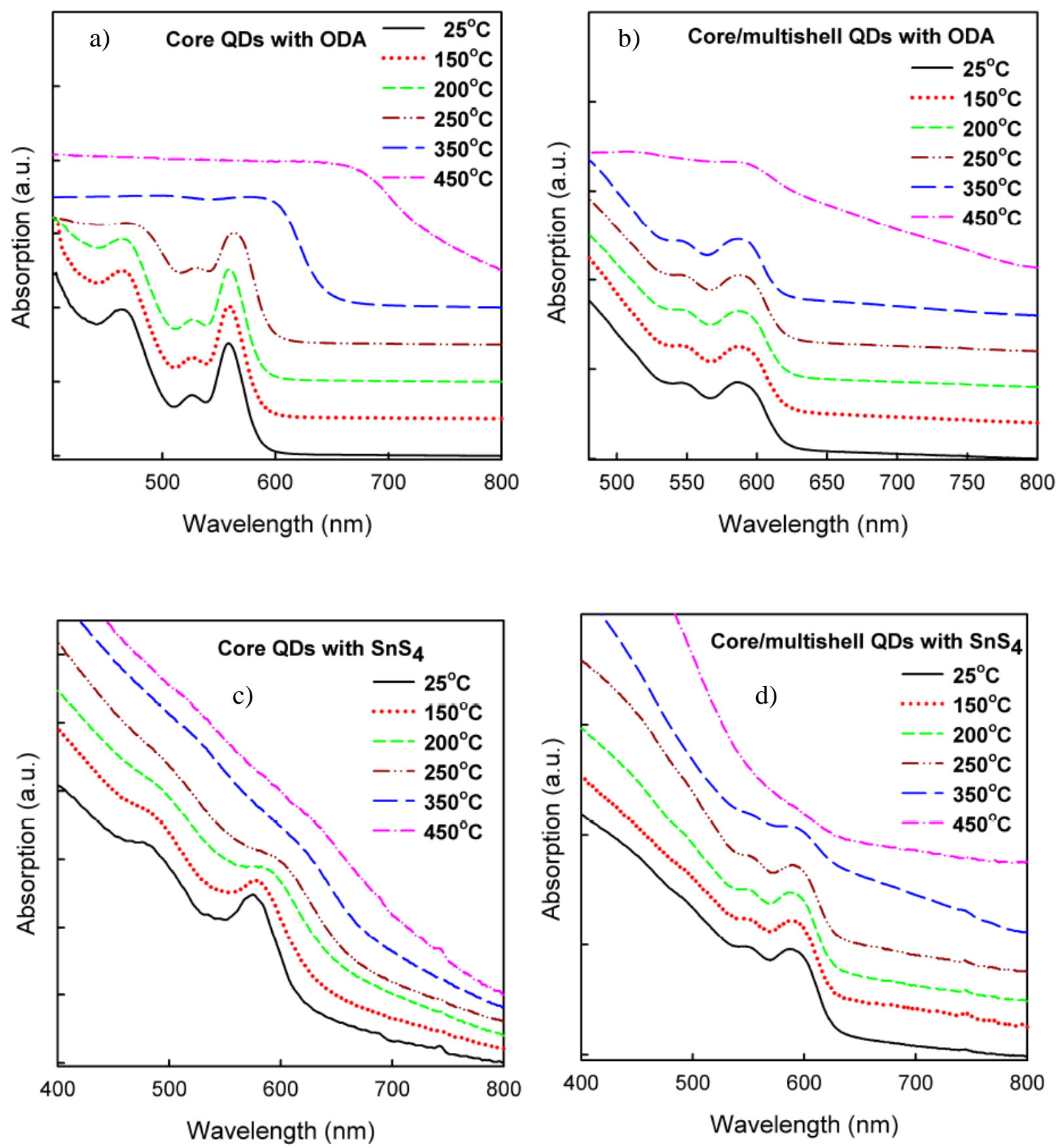

Figure 4.12 Absorption spectra of the films of ODA-capped (a) CdSe core QDs and (b) CdSe/CdS/ZnS core/multishell QDs as well as $\mathrm{SnS}_{4}$-capped (c) CdSe core QDs and (d) CdSe/CdS/ZnS core/multishell QDs annealed at different temperatures. The spectra are shifted in the vertical direction for clarity.

To investigate the thermal stability of the QDs and ligands, the QD films were annealed at $150-450{ }^{\circ} \mathrm{C}$ and then optically characterized. Figure 4.12 shows the evolution of the absorption 
spectra of the CdSe core QDs and CdSe/CdS/ZnS core/multishell QDs with $\mathrm{SnS}_{4}$ ligands or ODA ligands. For the CdSe core QDs with organic ligands (Figure 4.12 (a)), the excitonic features can be well resolved up to $250{ }^{\circ} \mathrm{C}$, but disappear after $350{ }^{\circ} \mathrm{C}$ anneal. This is evidence that the QDs remain physically distinct after annealing below $350{ }^{\circ} \mathrm{C}$, and are sintered during annealing above $350{ }^{\circ} \mathrm{C}$. The $450{ }^{\circ} \mathrm{C}$ annealed QD film has an absorption edge close to that of bulk CdSe $(\sim 690 \mathrm{~nm})$. As the annealing temperature is increased from $25-250{ }^{\circ} \mathrm{C}$, there is small red shift and broadening of the absorption peaks, which can be attributed to a decreased separation between annealed QDs. ${ }^{[86]}$ In contrast, for the ODA-capped CdSe/CdS/ZnS core/multishell QDs (Figure 4.12 (b)), the excitonic absorption peak can be retained up to $350{ }^{\circ} \mathrm{C}$. The absorption peak positions and shapes with the increasing annealing temperature from 25-250 ${ }^{\circ} \mathrm{C}$ are constant without any noticeable red shift or broadening. The strong thermal stability of core/multishell QDs results from the additional protection of CdS/ZnS multishell.

Figure 4.12 (c) and (d) shows the evolution of the absorption spectra of the CdSe core QDs and CdSe/CdS/ZnS core/multishell QDs with $\mathrm{SnS}_{4}$ ligands. As the annealing temperature is increased, the absorption spectrum of the core QDs shifts to the red and the excitonic peaks broaden. These changes are usually attributed to a decreased separation between annealed QDs. ${ }^{[86]}$ However, we have found that the peak shift and broadening are considerably larger than those of annealed core QDs with organic ligands. An additional factor which may contribute to the spectral changes is the transformation of the inorganic ligands upon annealing. ${ }^{[88]}$ First, water molecules solvating $\left(\mathrm{NH}_{4}\right)_{4} \mathrm{SnS}_{4}$ desorb after mild treatment between 100-200 ${ }^{\circ} \mathrm{C}$. Second, $\left(\mathrm{NH}_{4}\right)_{4} \mathrm{SnS}_{4}$ may decompose at $\sim 200{ }^{\circ} \mathrm{C}$ and transform into an amorphous or even crystalline semiconducting $\mathrm{SnS}_{2}$ phase, leading to stronger electronic coupling between QDs. Additionally, the change in the dielectric environment around the QDs arising from the ligand transformation may also cause a peak shift. ${ }^{[86]}$ As seen from Figure 4.12 (c), the excitonic features of the core QDs disappear after annealing at 350 and $450{ }^{\circ} \mathrm{C}$, suggesting that the CdSe QDs are no more distinct due to sintering at these temperatures. The annealed QD film exhibit an absorption edge 
close to that of bulk CdSe ( 690 nm). As seen from Figure 4.12 (d), the excitonic features of the core/multishell QDs can be resolved up to $350{ }^{\circ} \mathrm{C}$. This is evidence that the CdS/ZnS multishell improves the thermal stability of the cores, which remain physically instinct at $350{ }^{\circ} \mathrm{C}$. In addition, the excitonic peaks show minimal shift and broadening after annealing, suggesting that the optical properties of the QDs are mainly determined by core/shell structure, and less affected by the surface chemistry. This is also verified by the observation that the absorption spectrum of CdSe/CdS/ZnS core/multishell QDs with ODA ligands remained nearly constant after thermal treatments below $450{ }^{\circ} \mathrm{C}$, during which the ODA decomposed into a carbonaceous substance (Figure $4.12(\mathrm{~b})$ ).

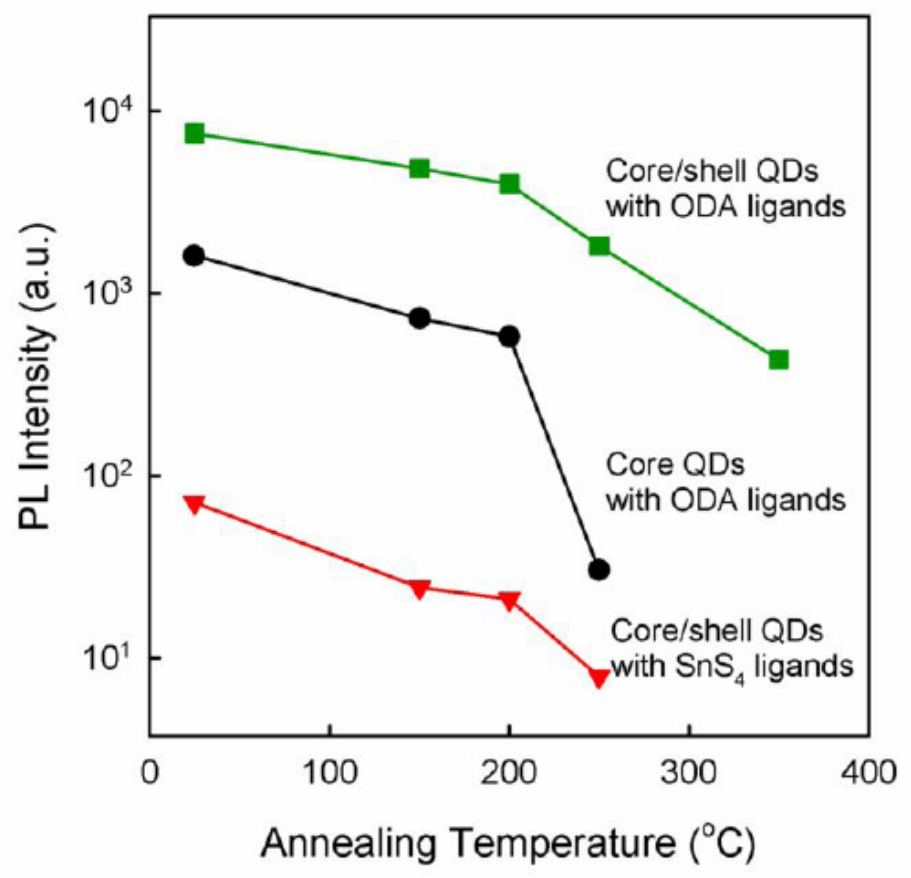

Figure 4.13 PL intensity of the films of CdSe core QDs and CdSe/CdS/ZnS core/multishell QDs with different ligands as a function of annealing temperature.

The PL of the $\mathrm{SnS}_{4}$-capped core QD film is completely quenched due to enhanced carrier delocalization and surface recombination. The variations of the PL intensity of the other three types of QD films with the annealing temperature are plotted in Figure 4.13. The PL of both the core QDs with organic ligands and the core/multishell QDs with inorganic ligands is completely 
quenched after annealing at temperatures above $250{ }^{\circ} \mathrm{C}$, but for different reasons. The former is ascribed to QD sintering, whereas the latter is due to the transformation of $\mathrm{SnS}_{4}$ into a more conductive phase, enabling more efficient electronic coupling. ${ }^{[46]}$ As seen, the PL of the core/multishell QDs with organic ligands can be measured as long as the QDs remain instinct, up to $350{ }^{\circ} \mathrm{C}$. The ODA-to-SnS 4 ligand exchange leads to a reduction in the PL intensity of the asdeposited film by a factor of $\sim 100$, even though the PL QY in solution dropped only by half. This finding evidences the remarkable effect of the surface chemistry on the PL of QD solids. The $\mathrm{SnS}_{4}$ ligands form an inorganic interface between QDs, so excitons may diffuse efficiently through the all-inorganic QD solid and experience nonradiative decay at quenching sites, ${ }^{[45]}$ causing pronounced PL quenching.

\subsection{Conclusions}

In summary, the ligand exchange of ODA-capped CdSe core and core/shell QDs with $\mathrm{Sn}_{2} \mathrm{~S}_{6}{ }^{4-}$ ligands was conducted in hydrazine solvent, leading to stable colloidal solutions. High colloidal stability was achieved due to the electrostatic interaction provided by the two terminal negatively charged sulfur atoms. Unfortunately, QDs could not retain their superior optical properties in hydrazine solvent. Furthermore, a similar ligand exchange of ODA-capped QDs with $\mathrm{SnS}_{4}$ ligands was conducted in a more benign aqueous $\mathrm{NH}_{4} \mathrm{OH}$ solution, which can help to largely preserve the high PL QYs of core/shell QDs. The applicability of $\mathrm{SnS}_{4}$-capped CdSe QDs thin films for optoelectronic applications is evaluated through detailed optical characterization before and after the ligand exchange. Exchange of the original organic ligands with small inorganic $\mathrm{SnS}_{4}$ ligands resulted in enhanced exciton delocalization and inter-QD electronic coupling, as evidenced by the redshifts and peak broadening of the absorption and PL spectra. The QDs with inorganic ligands retained strong excitonic absorption, but suffered significant PL quenching. Mild thermal treatment at temperatures lower than the sintering temperature was found to transform the ligands into a more conductive phase, linking individual QDs into an 
assembly of strongly-coupled functional QD blocks. The enhanced inter-QD coupling is expected to facilitate exciton dissociation and charge transport, and thus increase the QY of photocurrent generation. ${ }^{[45]}$ The current study suggests that nanocrystal QDs with metal chalcogenide ligands may be better suited for solar energy conversion in solar cells than use in light-emitting devices as luminophores. 


\section{Chapter 5 Quantum Dots with Inorganic Ligands for}

\section{Energy Conversions}

Monodispersed colloidal QDs synthesized by low-cost solution methods have many attractive properties, including high wavelength tunability, efficient luminescence, narrow emission bandwidth, and strong broadband absorption. They therefore offer a new class of materials for optoelectronic applications, ${ }^{[11,}$ 15, 89] such as light-emitting diodes (LEDs), photodetectors, and thin-film solar cells. However, the performance of QD-based devices is lagging significantly behind that of devices based on conventional bulk materials. For instance, the power conversion efficiencies of both QD-based LEDs and solar cells reported in the literature were only a few percent, ${ }^{[11,15,89]}$ well below those of the state-of-the-art inorganic and organic semiconductor devices.

The performance of QD-based devices largely depends on the surface properties of the QDs. Surface defects trap carriers and enhance the nonradiative recombination rate. Longhydrocarbon-chain organic ligands introduced for dispersion during colloidal synthesis may passivate most of the surface defects, but increase the interparticle spacing and energy barriers for electronic coupling. ${ }^{[15]}$ As a result, charge transport and exciton energy transfer between QDs and surrounding materials, two critical processes in optoelectronic device operation, are hampered. Complete removal of the organic ligands by different treatments has proven to be difficult and inevitably generates surface defects, deteriorating the optical properties of QDs. ${ }^{[3,90]}$ Simple exchange of the ligands with small thiol molecules is another commonly used approach, but the small organic molecules are not stable against oxidation and volatility. ${ }^{[44,91]}$

In an effort to seek ideal ligands which provide colloidal stabilization and electronic passivation of colloidal QDs, as well as enable facile inter-particle electronic communication, two groups recently exploited the exchange of bulky organic ligands with small inorganic ligands 
such as molecular metal chalcogenide complexes, ${ }^{[46,}$ 49] metal-free chalcogenide, ${ }^{[48]}$ hydrochalcogenide, ${ }^{[48]}$ and halide cations ${ }^{[92]}$. The sizes of these inorganic ligands are in the range of $0.1-0.7 \mathrm{~nm}$, significantly smaller than those of traditional long-chain organic ligands like octadecylamine (ODA) and trioctylphosphine oxide (TOPO). In various nanocrystal QD solids, the organic-to-inorganic ligand exchange greatly improved the inter-particle electronic coupling and led to facile charge transport. ${ }^{[46,48-49,92]}$ Even compared to the best results obtained from similar QDs with short-chain organic capping layers such as mercaptopropionic acid (MPA) and 1,2-ethanedithiol (EDT), the electron mobilities in such all-inorganic QD films were more than one order of magnitude higher. ${ }^{[49,92]}$ However, little effort has been made to explore the optoelectronic applications of these inorganically-capped QDs.

In this Chapter, we will carry out a systematic study of the electronic and optoelectronic properties of $\mathrm{SnS}_{4}$-capped CdSe core and core/shell QDs, and explore their applicability for efficient energy conversions in QD-based LEDs and solar cells. The QDs are synthesized by the hot injection method and subsequent organic-to-inorganic ligand exchange process developed in Chapter 2 and Chapter 4, respectively.

\subsection{CdSe Based Core/Multishell QDs with MCC Ligands for Light Emission}

The layer-by-layer (LBL) assembly method has proven to be one the most promising and practicable approaches to build up homogenous ultrathin films. ${ }^{[93]}$ In contrast to conventional deposition processes, the LBL method employs specific interaction forces between each deposited layer and therefore provides uniform and homogenous films with well defined internal structures over large areas. ${ }^{[84,94]}$ Herein, the LBL method is employed to fabricate QD-LEDs comprising $\mathrm{SnS}_{4}$-capped QD/polyelectrolyte multilayer films.

Firstly, ODA-capped CdSe/CdS/Zn ${ }_{0.5} \mathrm{Cd}_{0.5} \mathrm{~S} / \mathrm{ZnS}$ core/multishell QDs (PL QY = $65 \%$ ) 
were synthesized by the colloidal process as described in Chapter 2. Then, $\mathrm{SnS}_{4}$-capped $\mathrm{CdSe} / \mathrm{CdS} / \mathrm{Zn}_{0.5} \mathrm{Cd}_{0.5} \mathrm{~S} / \mathrm{ZnS}$ core/multishell QDs $(\mathrm{QY}=\sim 30 \%)$ were prepared through ligand exchange in $\mathrm{NH}_{4} \mathrm{OH}$, which has described in Chapter 4. After being washed with hexane 3 times, filtered, precipitated by adding acetonitrile ( $1: 1 \mathrm{v} / \mathrm{v})$, and centrifuged, $\mathrm{SnS}_{4}$-capped QDs were redissolved in formamide solvent. To fabricate QD-LEDs based on $\mathrm{SnS}_{4}$-capped QD/polyelectrolyte multilayer films, poly(diallydimethylammonium chloride) (PDDA), a cationic polyelectrolyte, was employed as the inter-dot linker. ${ }^{[94]}$ The negatively charged $\mathrm{SnS}_{4^{-}}$ capped CdSe/CdS/ $/ n_{0.5} \mathrm{Cd}_{0.5} \mathrm{~S} / \mathrm{ZnS}$ core/multishell QDs can be bonded to the positively charged PDDA, and assembled layer by layer into a uniform and homogeneous film.

The LEDs were fabricated on glass substrates with pre-patterned indium-tin-oxide (ITO) (sheet resistance $\sim 15 \Omega$ b). The substrates were sequentially cleaned in acetone, ethanol, and deionized (DI) water, and exposed to oxygen plasma (50W) for 20 min to fully hydrophilize the surface. Then, all fabrication steps were carried out in $\mathrm{N}_{2}$-filled glovebox. The ITO substrates were immersed into a $0.5 \mathrm{wt} \%$ aqueous solution of PDDA $(\mathrm{pH}=8)$ for $15 \mathrm{~min}$, rinsed twice with DI water (30 sec for each dipping) and then immersed in a $\mathrm{SnS}_{4}$-capped $\mathrm{CdSe} / \mathrm{CdS} / \mathrm{Zn}_{0.5} \mathrm{Cd}_{0.5} \mathrm{~S} / \mathrm{ZnS}$ core/multishell QDs/formamide solution $(0.5-1 \mathrm{mg} / \mathrm{mL})$ for $15 \mathrm{~min}$, and finally rinsed with pure formamide for $30 \mathrm{sec}$, and DI water for $30 \mathrm{sec}$. The above procedure was repeated for different times to form 5-30 bilayers of (PDDA/QDs). After annealing at $80{ }^{\circ} \mathrm{C}$ for $30 \mathrm{~min}$, the samples were then transferred from a glovebox to a high-vacuum thermal evaporation system. A $0.5 \mathrm{~nm} \mathrm{LiF}$ and $120 \mathrm{~nm} \mathrm{Al}$ cathode were deposited atop the LBL assembly film through a shadow mask. The whole fabrication is shown in Figure 5.1. All the QD-LEDs had an emitting area of $0.09 \mathrm{~cm}^{2}$.

The QD-LEDs were encapsulated with glass lids in $\mathrm{N}_{2}$ and characterized in air at room temperature. The electrical characteristics were measured using an Agilent 4156C semiconductor analyzer. The EL spectra were recorded using a silicon photodiode-array fiber-optic spectrometer. To calculate EQE, the QD-LEDs were placed directly on the surface of a calibrated silicon 
photodetector and all emitted photons from the glass side were captured.

Step 1

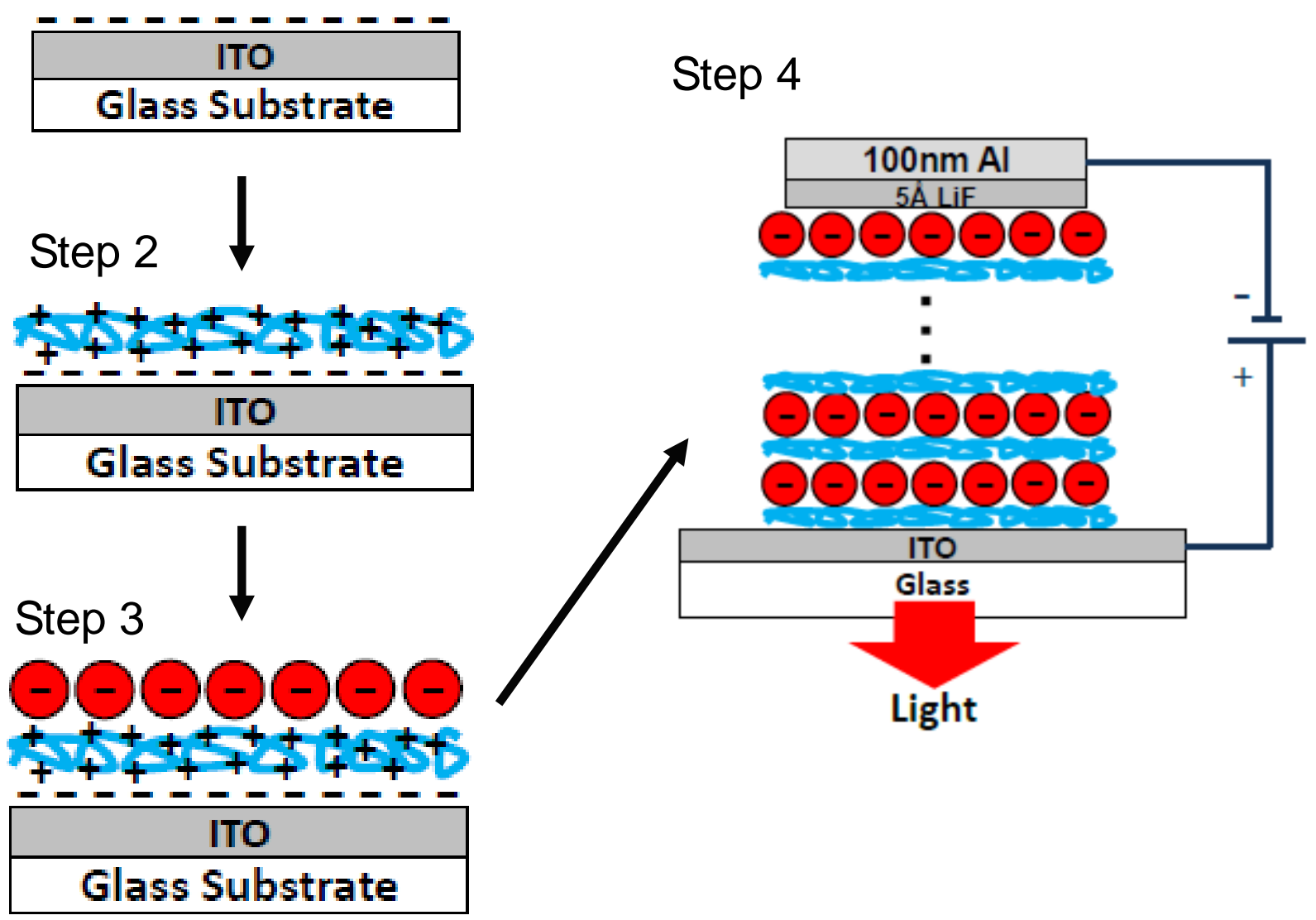

Figure 5.1 Scheme of the LBL assembly method to fabricate QD-LEDs.

Step 1 represents oxygen plasma treatment (20 min) to generate a fully hydrophilized negative-charged surface. Step 2 represents the adsorption of positive-charged PDDA polyelectrolyte, following by rinsing with DI water. Step 3 represents the adsorption of negative-charged $\mathrm{SnS}_{4}$-capped QDs, following by rinsing with formamide and DI water. The steps 1-3 were repeated to form 5-30 bilayers of (PDDA/QDs). Step 4 represents the final thermal-evaporating deposition of $\mathrm{LiF}$ and $\mathrm{Al}$ electrode.

A good linear relationship between the absorbance and number of bilayers of the films was obtained for $\mathrm{SnS}_{4}$-capped QDs/PDDA from the LBL assembly method. A set of absorption spectra of $\mathrm{SnS}_{4}$-capped QDs/PDDA films of different thickness is presented in Figure 5.2 (a). As seen from the enlarged absorption spectra in the inset, $\mathrm{SnS}_{4}$-capped $\mathrm{CdSe} / \mathrm{CdS} / \mathrm{Zn}_{0.5} \mathrm{Cd}_{0.5} \mathrm{~S} / \mathrm{ZnS}$ core/multishell QDs retain their size-quantized absorption spectra with the discrete excitonic 
absorption peaks at the same wavelengths as in the case of the colloidal solution. A linear increment of the absorbance against the number of bilayers (Figure 5.2 (b)) suggests that an equal number of QDs was deposited during each LBL assembly cycle.
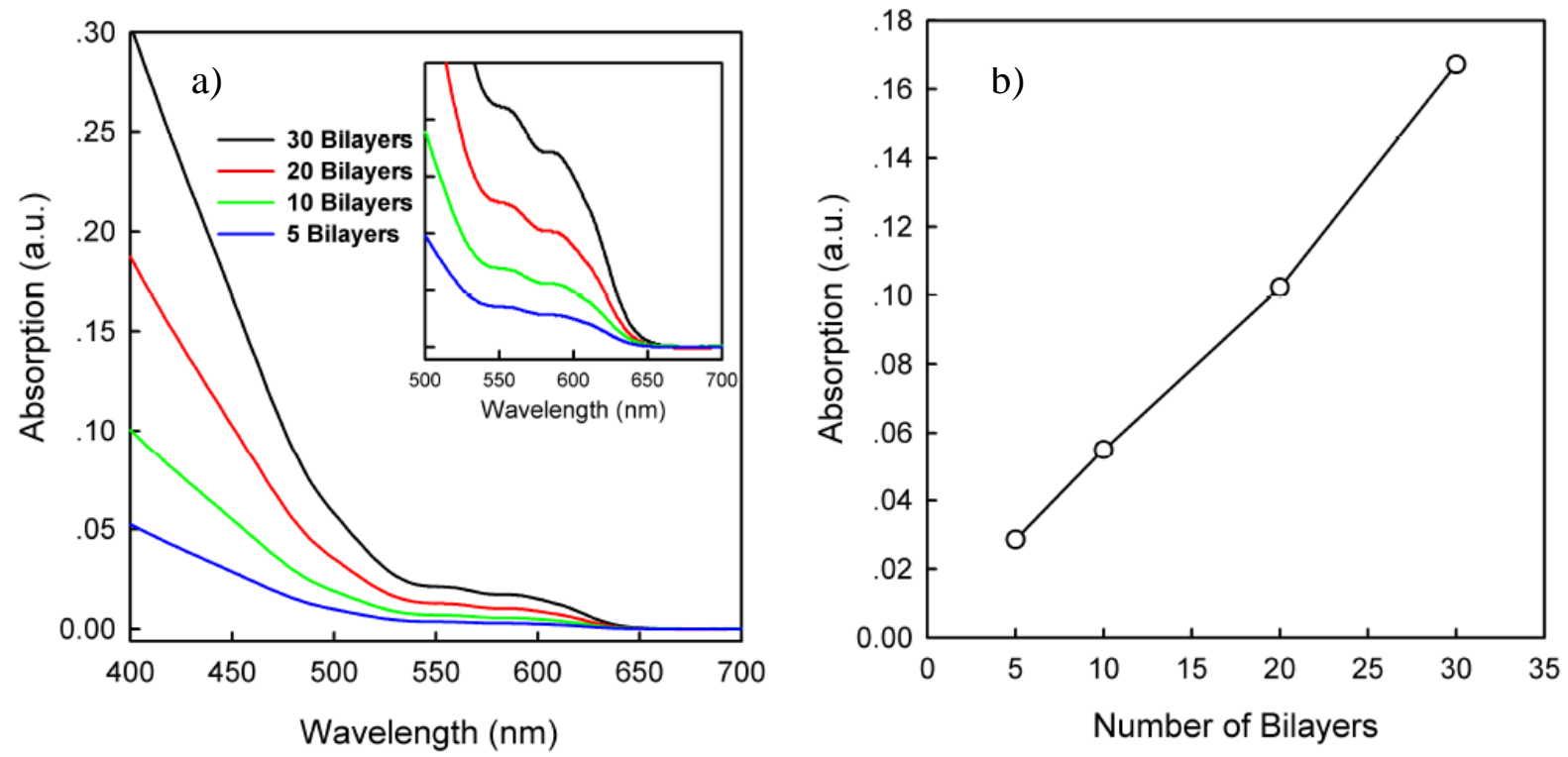

Figure 5.2 (a) Absorption spectra of 5, 10, 20, and 30 bilayers of $\mathrm{SnS}_{4}$-capped QDs/PDDA films. The discrete excitonic absorption peaks can be seen from the inset of enlarged absorption spectra. (b) Absorbance at the shoulder position of $450 \mathrm{~nm}$ vs. the number of QDs/PDDA billayers.

The electrical characteristics of the QD-LEDs are similar to those of a typical diode. The forward I-V characteristics of a representive QD-LED are shown in Figure 5.3(a). The device has a low turn-on voltage of 4V. Unfortunately, the QD-LEDs based on $\mathrm{SnS}_{4}$-capped QDs exhibited very low light emission, with an EQE $~ 0.001 \%$. The EL spectrum of a QD-LED comprising of 10 bilayers of $\mathrm{SnS}_{4}$-capped QDs/PDDA taken at $100 \mathrm{~mA} / \mathrm{cm} 2$ is shown in Figure 5.3(b), along with a PL spectrum of the same QD film. The EL spectrum resembles the PL spectrum, confirming that the EL indeed originates from the QDs. However, due to the low intensity of the emission from the QD-LED, the curve of the EL spectrum is quite noisy. 

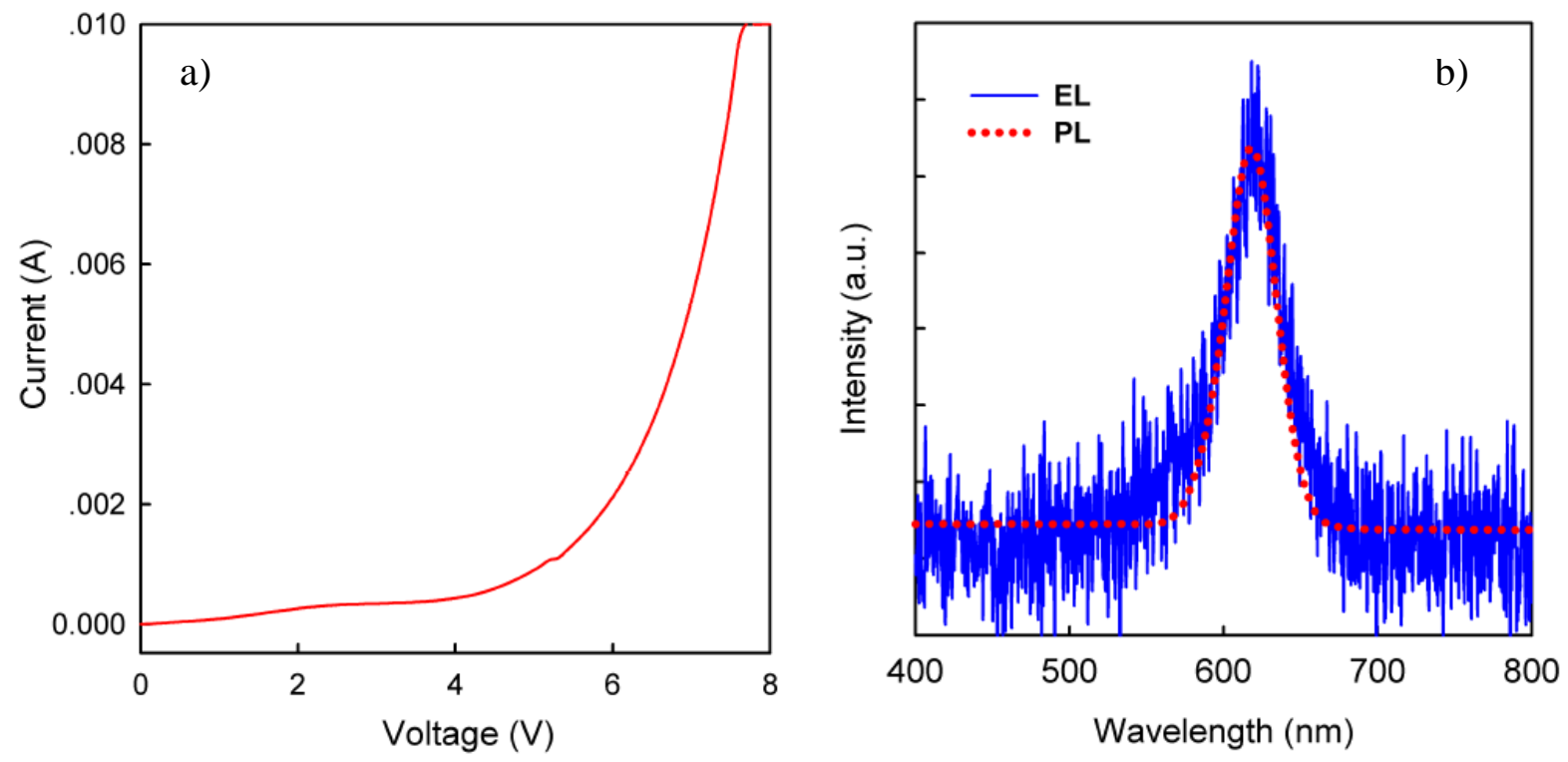

Figure 5.3 The best device performance of QD-LEDs comprising of 10 bilayers of $\mathrm{SnS}_{4}$-capped QDs/PDDA films. (a) I-V Characteristics and (b) normalized EL spectrum taken at $100 \mathrm{~mA} / \mathrm{cm}^{2}$ and PL spectrum of 10 bilayers of $\mathrm{SnS}_{4}$-capped QDs/PDDA films.

The poor performance of QD-LEDs may be attributed to a combination of: (1) the $\mathrm{SnS}_{4}$ ligands can not efficiently passivate the QD surfaces in QD solids, leaving dangling bonds on the QD surfaces as nonradiative recombination sites; (2) leakage of carriers to the $\mathrm{SnS}_{4}$ capping layer leads to reduced overlap between the electron and hole wave functions and thus reduced radiative exciton decay inside the QDs; and (3) excitons may diffuse fast through the allinorganic QD layers and more likely experience nonradiative decay at quenching sits, even with the multishell protection. Therefore, the device result is consistent with our previous optical evaluation of $\mathrm{SnS}_{4}$-capped QD solution and solids (Chapter 4), and suggests that QDs with metal chalcogenide ligands are not suited for use in LEDs as luminophores.

\subsection{Quantum Dots with MCC Ligands for Photovoltaic Conversion}

To evalue the applicability of CdSe QDs with different lignads for solar energy conversion, we fabricated simple ITO/QDs/Al structures as shown in Figure 5.4, and measured 
their electrical characteristics and photocurrent response. This simple sandwich structures allow measurements of the electrical conductivity as well as photoconductivity in the QD films since a Schottky barrier may form at the QD/Al junction under illumination. ${ }^{[38]}$

First, QDs with organic and inorganic ligands were synthesized. CdSe core QDs with a diameter of $\sim 3.2 \mathrm{~nm}$, capped with organic ODA ligands, were synthesized using the conventional hot-injection method. ${ }^{[15]}$ The QDs were subjected to a multistep precipitation and redissolvation process of purification to remove side products and unreacted precursors. The as-synthesized QDs dispersed in hexane had a PL QY of 10\%. Molecular metal chalcogenide was prepared by dissolving $\mathrm{SnS}_{2}$ in aqueous $\mathrm{Na}_{2} \mathrm{~S}$, forming $\mathrm{Na}_{4} \mathrm{SnS}_{4}$, which were precipitated and moderately annealed at $\sim 80{ }^{\circ} \mathrm{C}$ to remove the residual solvent. To perform the ligand exchange, $\mathrm{Na}_{4} \mathrm{SnS}_{4}$ dissolved in aqueous $\mathrm{NH}_{4} \mathrm{OH}$ was mixed with CdSe QDs dispersed in hexane, and stirred until all the QDs were transferred from the nonpolar organic medium to the polar aqueous solvent. During this step, the organic ligands were stripped off from the QD surfaces, leaving behind electron-deficient metal atoms with a strong affinity toward bonding with $\mathrm{S}$ in $\mathrm{SnS}_{4}{ }_{4}{ }^{4}$ ions that were neutralized mainly by $\mathrm{NH}_{4}{ }^{+}$counterions. ${ }^{[46]}$ The negatively charged $\mathrm{SnS}_{4}$ ligands prevented aggregation of the QDs, resulting in a stable colloidal solution of QDs. The QDs were then filtered through a $0.2 \mu \mathrm{m}$ PVDF filter, precipitated by adding acetonitrile $(1: 1 \mathrm{v} / \mathrm{v})$, centrifuged, and re-dissolved in $\mathrm{NH}_{4} \mathrm{OH}$.

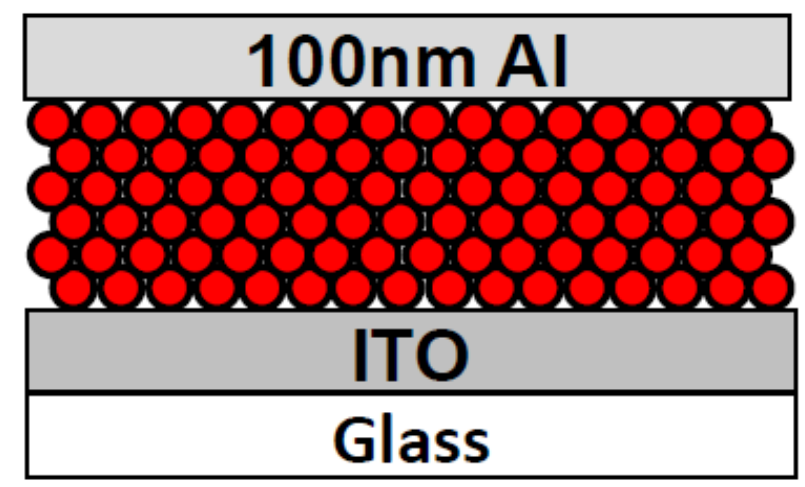

Figure 5.4 Cross-section of an ITO/CdSe QDs/Al structure.

Next, QD thin films 100 nm thick, composed of disordered arrays of closely-packed 
QDs, were prepared on ITO/glass by spin-coating from concentrated solutions of ODA-capped QDs in hexane ( $100 \mathrm{mg} / \mathrm{ml})$, or $\mathrm{SnS}_{4}$-capped QDs in hydrazine $(\sim 25 \mathrm{mg} / \mathrm{ml})$. The hydrazine solvent was used because it can produce much more uniform QD thin films than $\mathrm{NH}_{4} \mathrm{OH}$. Before loading the samples into a high-vacuum thermal evaporator, the QD films were first baked at 80 ${ }^{\circ} \mathrm{C}$ for $30 \mathrm{~min}$, and then annealed in $\mathrm{N}_{2}$ at $150-450{ }^{\circ} \mathrm{C}$ for $5 \mathrm{~min}$. An aluminum electrode $(\sim 100$ $\mathrm{nm}$ ) was deposited on the QDs films through a shadow mask with $3 \mathrm{~mm} \times 3 \mathrm{~mm}$ square openings. The electrical characteristics of the structures were measured using an Agilent 4156C semiconductor analyzer. Photocurrent responses were measured using a $150 \mathrm{~W}$ Xenon lamp source through a monochromator which was synchronized with an Agilent 4156C semiconductor analyzer.

\subsubsection{Electrical Characterization of ITO/MCC-capped QDs/Al}

Figure 5.5 compares the I-V characteristics of the devices with organically and inorganically-capped CdSe QDs which were subjected to annealing in $\mathrm{N}_{2}$ at different temperatures prior to the deposition of Al. In both devices, the current increases steadily with increasing annealing temperature, implying increased charge mobility in the QD solids. The device with ODA-capped CdSe QDs exhibits leak Schottky characteristics which is nearly symmetric, indicating similar electron injection efficiencies from the ITO and Al electrodes. In contrast, the I-Vs of the device with $\mathrm{SnS}_{4}$-capped CdSe QDs are almost linear even without annealing, indicating the ohmic nature of both interfaces between the QDs and electrodes. For the unannealed device, the ligand exchange resulted in an increase in electrical conductivity by six orders of magnitude. After annealing at $450{ }^{\circ} \mathrm{C}$, the difference drops to one order of magnitude. These findings confirm enhanced electronic coupling between $\mathrm{SnS}_{4}$-capped QDs, as inferred from the above absorption data. We have also found that, while adding a ZnS shell can better preserve the optical properties, it introduces an additional barrier for charge tunneling, leading to a much lower conductivity in similar sandwich structures. 

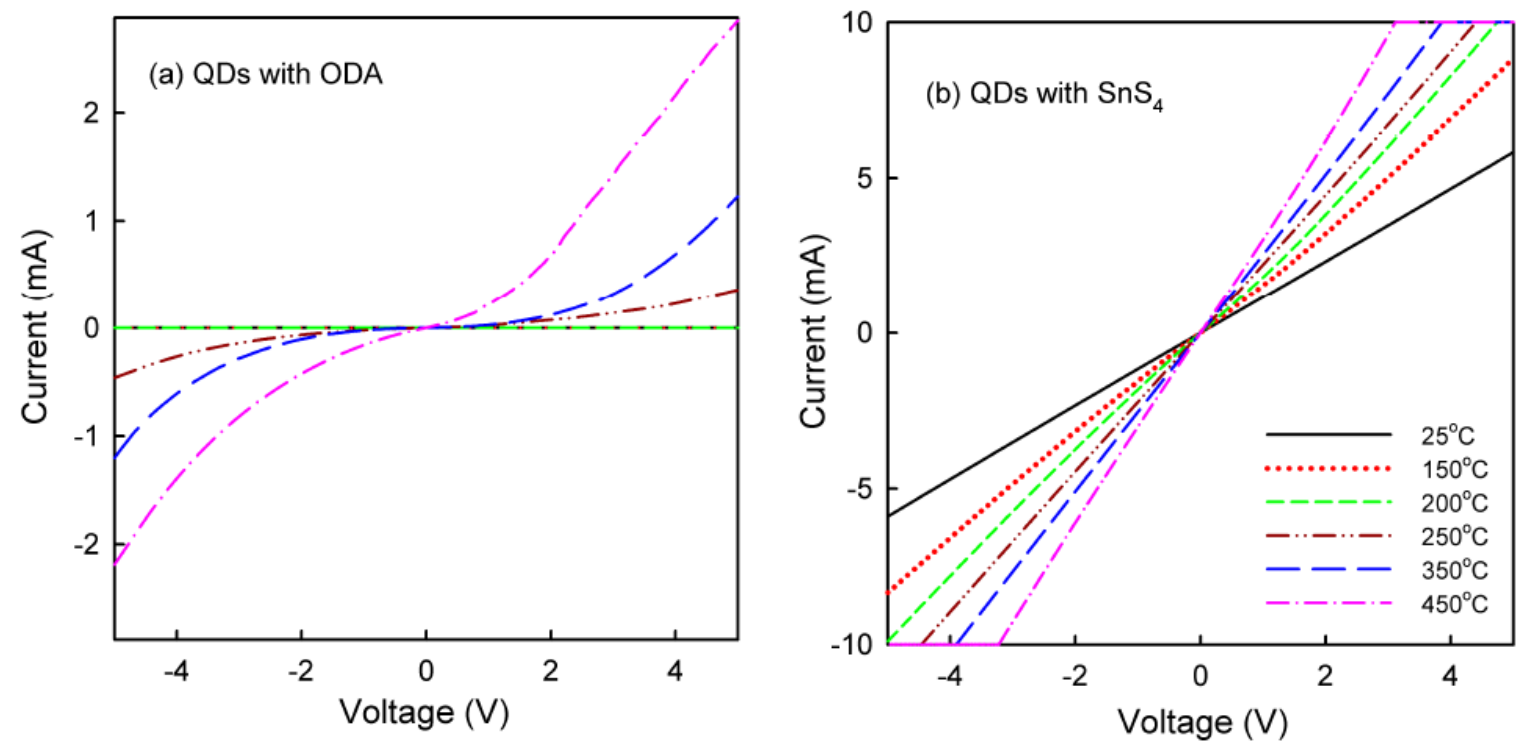

Figure 5.5 I-V characteristics of ITO/CdSe QDs/Al structures. The QDs, with (a) ODA and (b) $\mathrm{SnS}_{4}$ ligands, were annealed at different temperatures prior to $\mathrm{Al}$ deposition

The evolution of current in the $250{ }^{\circ} \mathrm{C}$-annealed devices measured at a constant bias of 5 $\mathrm{V}$ in the dark is shown in Figure 5.6. The current in the device with ODA-capped QDs decreases monotonically and drops to $67 \%$ of the initial value after the bias is held for $100 \mathrm{~s}$. The time-dependence of the current can be fit to a stretched exponential function (Kohlrausch function) with the stretching exponent $\beta \sim 0.28$, which indicates current decay at a slowing rate. Similar behaviors have been seen in CdSe QDs with TOPO ligands. ${ }^{[95]}$ It has been found that the current can be largely restored after removing the bias and keeping the sample in the dark for a long period of time. In contrast, the current in the device with $\mathrm{SnS}_{4}$-capped QDs is nearly constant and shows less than 1\% decay after $600 \mathrm{~s}$. The large current decay in the ODA-capped QDs can be explained by space-charge limited current. ${ }^{[95]}$ In the QD solid, there exists a large number of trap states arising from dangling bonds at the QD surfaces and/or disordered nanostructures. Trapping of carriers at these states gives rise to space charge which reduces the internal field and injected current. The initial build-up of trapped charge causes a rapid drop of the current. As the traps are filled up, the trapping rate decreases and the current tends to stabilize. The absence of such space-charge limited current in the all-inorganic device, as seen in Figure 
5.6, suggests that space charge cannot build up in the $\mathrm{SnS}_{4}$-capped QD film. Similar phenomena were observed in devices with unannealed $\mathrm{SnS}_{4}$-capped QDs and those annealed below $200{ }^{\circ} \mathrm{C}$. It is plausible that, due to strong inter-QD electronic coupling and charge transport, it is hard to form insolated traps which are deep enough to immobilize carriers for a time longer than the measurement time. However, in the QDs film with ODA, the organic ligands form an insulating layer surrounding the QDs, facilitating the formation of deep isolated traps.

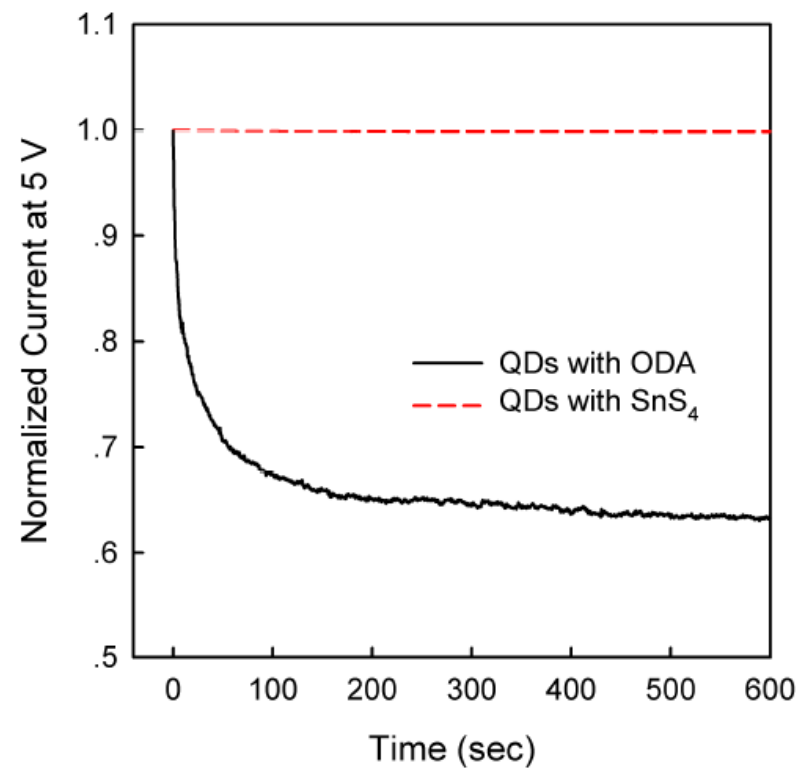

Figure 5.6 Evolution of current in ITO/CdSe QDs/Al structures under a constant bias of $5 \mathrm{~V}$. The QDs were annealed at $250{ }^{\circ} \mathrm{C}$ prior to $\mathrm{Al}$ deposition

Given efficient charge trapping in the ODA-capped QD film, we expect that the I-V curve of the device is strongly dependent on the testing history of the sample. Indeed, as seen in Figure 5.7 (a), scans under different conditions resulted in different I-V characteristics. The first scan represents the initial test of the as-fabricated device in the dark. As the scan was repeated, a large reduction in current was observed. The third scan shown in Figure 5.7 (a) was taken after the sample was biased at $5 \mathrm{~V}$ for $10 \mathrm{~min}$. At this point, it can be assumed that all traps are filled, and the data shows the steady-state space-charge limited current in the device. Finally, the device was illuminated with white light for $20 \mathrm{~s}$, and another test was taken. The illumination not only 
emptied the traps, but also photo-generated more free carriers which can persist for a certain amount of time, leading to a conductivity higher than the initial conductivity of the QD film. As expected, the $\mathrm{I}-\mathrm{V}$ characteristics of the as-fabricated $\mathrm{SnS}_{4}$-capped QD device are nearly independent of the sample history, as seen in Figure 5.7 (b).
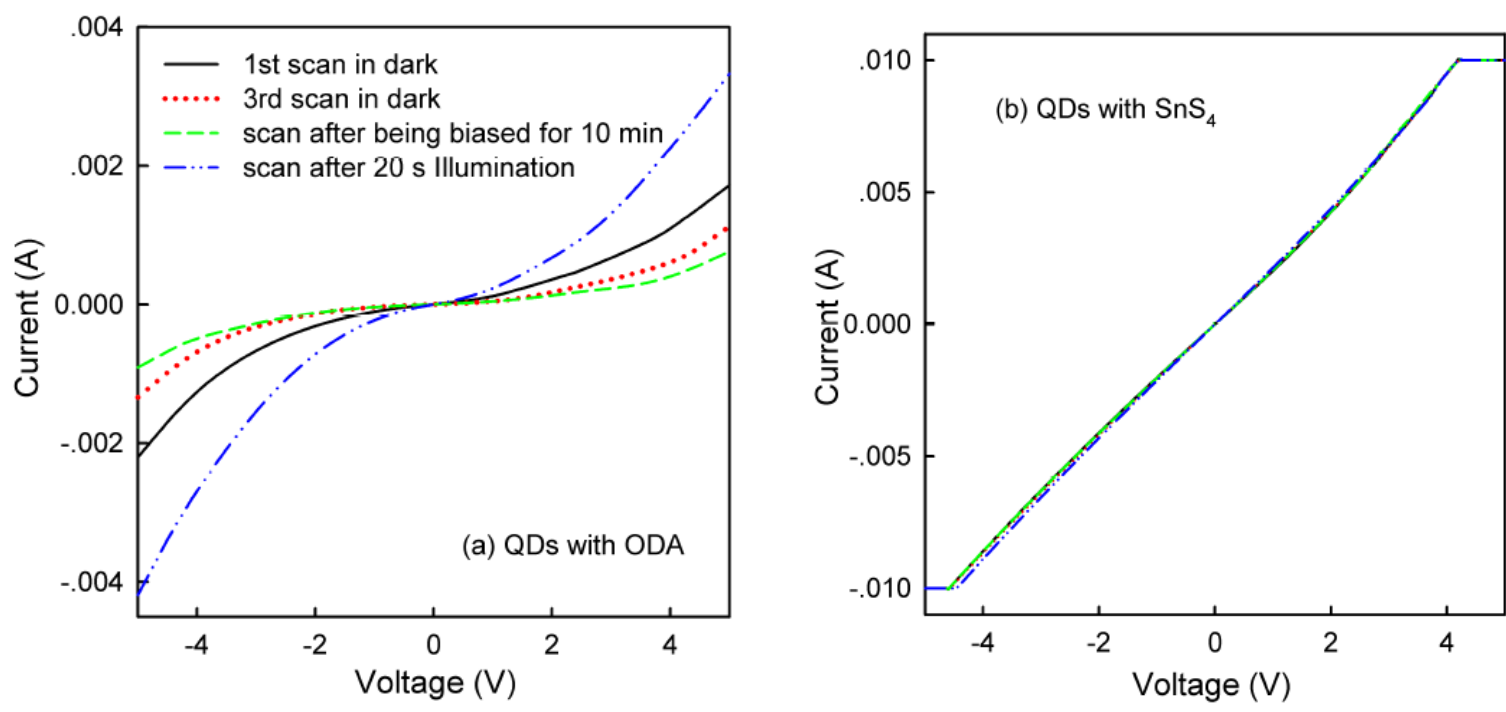

Figure 5.7 I-V characteristics of as-fabricated ITO/CdSe QDs/Al structures with (a) ODA and (b) $\mathrm{SnS}_{4^{-}}$ capped QDs, as a result of different scans.

\subsubsection{Photocurrent Response of ITO/MCC-capped QDs/Al}

For the unannealed devices and those annealed below $350{ }^{\circ} \mathrm{C}$, their spectral responses of photocurrent measured at zero bias closely followed their absorption spectra, suggesting that the photocurrents arose from exciton formation and dissociation in the QDs. An example is shown in Figure 5.8, where both devices annealed at $150{ }^{\circ} \mathrm{C}$ exhibit a maximum photocurrent at the first excitonic absorption peak. In the device with ODA-capped QDs, higher-order excitonic peaks can also be resolved. Another important finding is that the photocurrent in the device with $\mathrm{SnS}_{4}$ capped QDs is more than two orders of magnitude higher than that in the device with organic ligands. This, again, can be attributed to the $\mathrm{SnS}_{4}$ ligands which are responsible for faster exciton dissociation as well as more facile charge transport in the QD film. However, the peak external quantum efficiency (EQE) of this device is only 1-2\%. The poor yield of photocurrent generation 
may be partially attributed to the fact that only a low fraction ( 20\%) of light was absorbed by the thin QD film. More importantly, the open-circuit voltages we obtained were very small (tens of $\mathrm{mV}$ ), indicating that there is a lack of driving forces which can efficiently separate photogenerated excitons and drive electrons toward the $\mathrm{Al}$ electrode in the current device architecture. This is presumably due to the small difference between the Fermi levels of the QDs and $\mathrm{Al}$ or caused by Fermi energy spinning at the QD surfaces. We expect that, if a cathode metal with a lower work function is used, a larger Schottky barrier may form at the QD/cathode interface and act as an effective photocurrent generation zone. ${ }^{[38]}$ Alternatively, the CdSe QDs may be combined with CdTe QDs to form a type-II heterojunction, where photogenerated excitons can dissociate efficiently through donor-acceptor charge transfer. ${ }^{[96]}$ Such solar cell structures would benefit more from the facile charge transport through the inorganically-capped QD solids, and exhibit a much higher EQE. Nevertheless, the data in Figure 5.8 implies the potential applicability of QDs with metal chalcogenide ligands in thin-film photodetectors and solar cells for efficient energy conversions. Note that the same ligand exchange technique can also be applied to QDs with a smaller bandgap, like $\mathrm{PbX}(\mathrm{X}=\mathrm{S}, \mathrm{Se})$, which are more suitable for solar energy harvesting. ${ }^{[97]}$
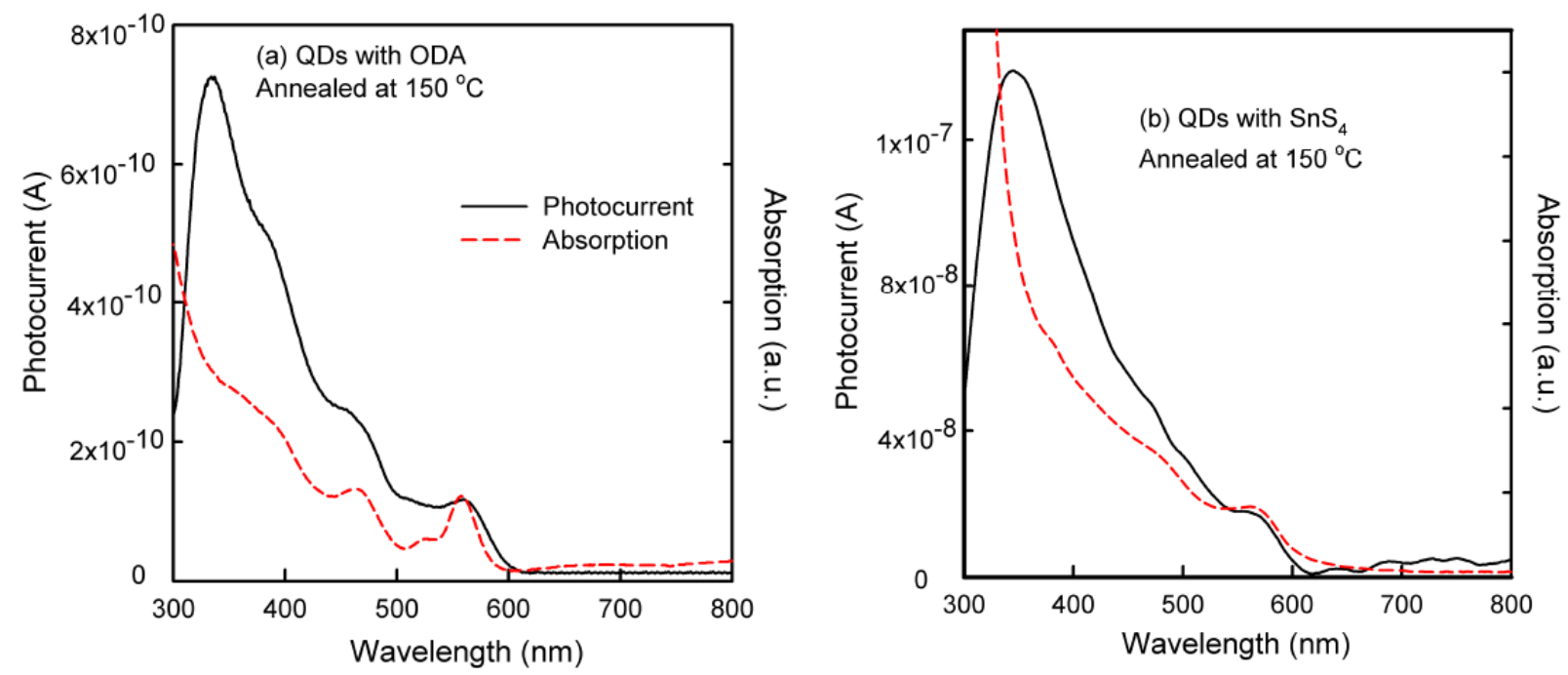

Figure 5.8 Photocurrent spectral responses and absorption spectra of ITO/CdSe QDs/Al structures comprising QDs with (a) ODA and (b) $\mathrm{SnS}_{4}$ ligands. The QDs were annealed at $150{ }^{\circ} \mathrm{C}$ prior to $\mathrm{Al}$ deposition 
Figure 5.9 shows the photocurrent responses of devices comprising CdSe QDs with organic and inorganic ligands, annealed at different temperatures. The photocurrent in the unannealed device with organic ligands is very low, on the order of $10^{-11} \mathrm{~A}$. It increases with increasing annealing temperature up to $350{ }^{\circ} \mathrm{C}$ due to improved charge transport, and then drops after $450{ }^{\circ} \mathrm{C}$ annealing due to reduced light absorption in sintered QDs. The peak photocurrent in the $350{ }^{\circ} \mathrm{C}$-annealed sample is increased by as much as four orders of magnitude compared to that in the unannealed sample. As seen in Figure 5.9 (b), the device with $\mathrm{SnS}_{4}$ ligands exhibits a different temperature-dependent behavior. The photocurrent reaches the maximum after $150{ }^{\circ} \mathrm{C}$ annealing, and drops substantially as the annealing temperature is further raised. Upon annealing above $200{ }^{\circ} \mathrm{C}$, the $\mathrm{SnS}_{4}$-capped QD film actually displays a lower photoconductivity than the film with organic ligands. We believe that the mechanisms underlying the photocurrent quenching in the former are the same as those responsible for PL quenching described earlier, i.e. relaxed quantum confinement and fast diffusion of carriers to defect sites, both caused by the inorganic ligands. Especially above $200{ }^{\circ} \mathrm{C}$, the transformation of $\mathrm{SnS}_{4}$ into to a more conductive phase can greatly degrade both absorption and photocurrent generation.
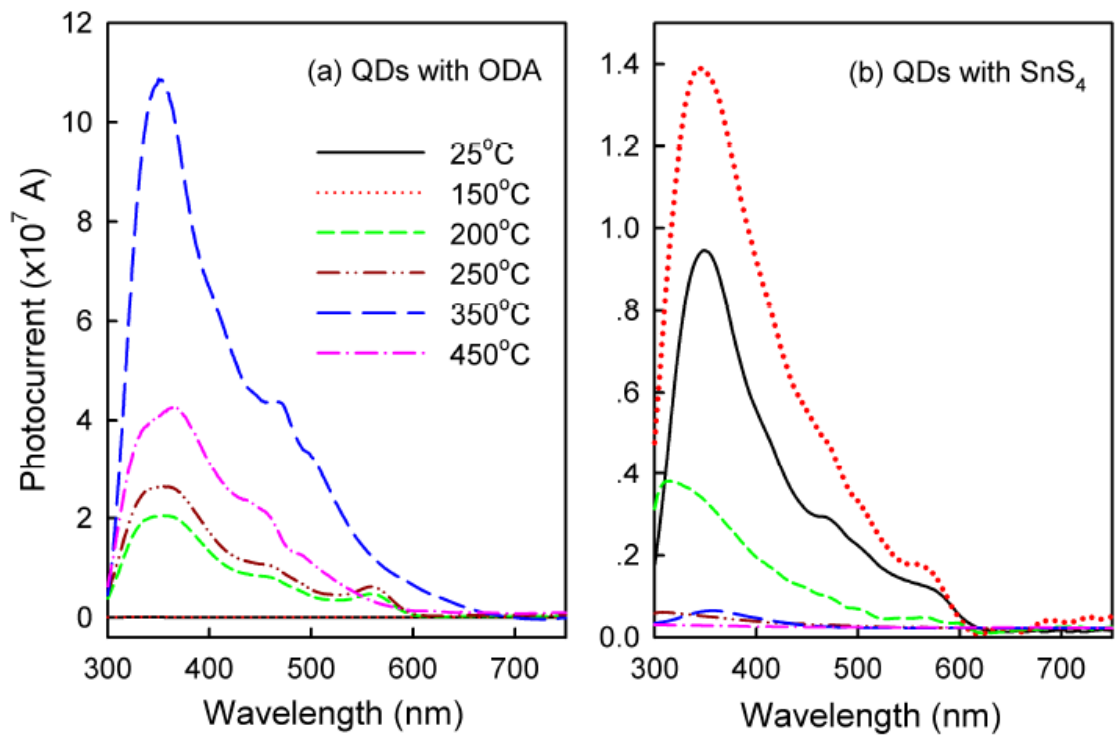

Figure 5.9 Photocurrent spectral responses of ITO/CdSe QDs/Al structures with (a) ODA and (b) $\mathrm{SnS}_{4}{ }^{-}$ capped QDs, which were annealed at different temperatures prior to Al deposition 


\subsection{Conclusions}

In summary, the effects of organic-to-inorganic ligand exchange on the electronic and optoelectronic properties of colloidal CdSe core and core/shell QDs were investigated. To evaluate of $\mathrm{SnS}_{4}$-capped QDs for light emission application, $\mathrm{SnS}_{4}$-capped $\mathrm{CdSe} / \mathrm{CdS} / \mathrm{Zn}_{0.5} \mathrm{Cd}_{0.5} \mathrm{~S} / \mathrm{ZnS}$ core/multishell QDs were assembled into films using LBL assembly with PDDA as the linker. The QD-LEDs were fabricated by sandwiching $\mathrm{SnS}_{4}$-capped QDs/PDDA films between ITO and aluminum electrodes. The LEDs had a low turn-on voltage of $\sim 4 \mathrm{~V}$ and stable EL matching well with the PL spectrum of the QD film. However, due to the strong conductivity and poor passivation properties of $\mathrm{SnS}_{4}$ ligands, the efficiency of QD-LEDs is significantly lower than similar LEDs based on CdSe QDs with original organic ligands. A simple ITO/SnS 4 -capped CdSe core QDs/Al structure was used to evaluate of $\mathrm{SnS}_{4}$-capped QDs for photovoltaic conversion. The devices showed much higher electrical conductivity and reduced space-charge limited current after the ligand exchange, indicating that the small inorganic $\mathrm{SnS}_{4}$ ligands resulted in enhanced inter-QD electronic coupling. Meanwhile, the $\mathrm{SnS}_{4}{ }^{-}$ capped QDs were found to retain strong excitonic absorption. Under illumination, the photocurrent response of an ITO/QDs/Al structure increased by several orders of magnitude after the ligand exchange. These findings bode well for the applicability of colloidal QDs with metal chalcogenide ligands as functional blocks to achieve efficient photodetection and solar energy conversion at low processing temperatures. 


\section{Chapter 6 Conclusions and Future Work}

\subsection{Conclusions}

This dissertation has focused on the synthesis, surface modification, and characterization of II-VI colloidal QDs, and evaluation of their suitability for high-efficiency energy conversions in LED and solar cell structures. Firstly, high-quality CdSe core and core/shell QDs were synthesized using low-cost colloidal chemical processes. Secondly, high-efficiency CdSe/ZnS QD-LEDs were demonstrated: phosphorescent exciton harvesters have been used to enhance

exciton energy transfer from charge-transport layers to QDs; high-efficiency QD-LEDs via Förster resonance energy transfer from phosphorescent molecules are achieved. Furthermore, organic ligands of QDs have been exchanged with novel inorganic ligands to improve interparticle electronic coupling in QD solids. The optical properties of $\mathrm{SnS}_{4}$-capped CdSe core and core/shell QDs were systematically characterized. Finally, $\mathrm{SnS}_{4}$-capped CdSe-based QDs for energy conversions (light emission and photovoltaic conversion) were evaluated.

The primary results of the research are summarized as the following:

1. Synthesis and characterization of colloidal semiconductor QDs (Chapter 2)

An in-house capability of colloidal QD synthesis by the hot-injection method was developed. Through tuning reaction time, the absorption spectrum and PL emission peak of QDs can be tuned over a wide range. High-quality CdSe, CdS and CdTe core QDs with sharp excitonic absorption features and narrow PL bandwidths were synthesized;

* CdSe/ZnS core/shell QDs were synthesized via the SILAR method. The highest PL QY 50\% was obtained after the growth of 3 monolayers of ZnS shell; 
In order to reduce the interfacial defects so as to further improve the PL QY, CdSe QDs with a graded $\mathrm{CdS} / \mathrm{Zn}_{0.5} \mathrm{Cd}_{0.5} \mathrm{~S} / \mathrm{ZnS}$ multishell were synthesized. A PL QY $\sim 65 \%$ was achieved;

2. Development of high-Efficiency CdSe/ZnS QD-LEDs (Chapter 3)

* CdSe/ZnS QD-LEDs were fabricated by integrating the QD emission layer into a hybrid ITO/PEDOT:PSS/poly-TPD/QD/BCP/Alq $/$ LiF/Al structure. The QD layer thickness was optimized by spin-coating QD solutions of different concentrations in the range of $1-10 \mathrm{mg} / \mathrm{ml}$. The best concentration of QD solutions was determined to be $3 \mathrm{mg} / \mathrm{ml}$, corresponding to $1.6 \mathrm{MLs}$ of green CdSe/ZnS QDs. Pure QD EL was achieved with a moderate turn-on voltage of $\sim 4 \mathrm{~V}$ from $3 \mathrm{mg} / \mathrm{ml}$ QD solution. The optimized QD-LED was used as the baseline device in our further work on performance enhancement;

* We demonstrated that the EL of green QD-LEDs can be significantly enhanced by using blue phosphorescent dyes as efficient exciton harvesters and energy donors. Precise control of the concentration of the FIrpic donors doped in a CBP host and their distance from the QD layer led to complete exciton energy transfer and EL enhancement by a factor of 2.5. The Förster distance between FIrpic molecules and green CdSe/ZnS QDs was determined to be 8-10 nm, in a good agreement with the value calculated using the Förster model. Our study shows that the integration of QDs with phosphorescent organic dyes can overcome the difficulty of charge injection into QDs with bulky organic ligands, and provide an effective route to improve the EQE of QD-based hybrid LEDs;

3. Synthesis and characterization of CdSe QDs with inorganic ligands (Chapter 4)

* The ligand exchange of ODA-capped CdSe core and core/shell QDs with $\mathrm{Sn}_{2} \mathrm{~S}_{6}{ }^{4-}$ ligands was conducted in hydrazine solvent, leading to stable colloidal solutions. High colloidal stability was achieved due to the electrostatic interaction provided 
by the two terminal negatively charged sulfur atoms. Unfortunately, QDs could not retain their superior optical properties in hydrazine solvent;

- A similar ligand exchange of ODA-capped QDs with $\mathrm{SnS}_{4}$ ligands was conducted in a more benign aqueous $\mathrm{NH}_{4} \mathrm{OH}$ solution, which can help to largely preserve the high PL QYs of core/shell QDs;

* The applicability of $\mathrm{SnS}_{4}$-capped CdSe QDs thin films for optoelectronic applications is evaluated through detailed optical characterization before and after the ligand exchange. Exchange of the original organic ligands with small inorganic $\mathrm{SnS}_{4}$ ligands resulted in enhanced exciton delocalization and inter-QD electronic coupling, as evidenced by the redshifts and peak broadening of the absorption and PL spectra. The QDs with inorganic ligands retained strong excitonic absorption, but suffered significant PL quenching. Mild thermal treatment at temperatures lower than the sintering temperature was found to transform the ligands into a more conductive phase, linking individual QDs into an assembly of strongly-coupled functional QD blocks. The enhanced inter-QD coupling is expected to facilitate exciton dissociation and charge transport, and thus increase the QY of photocurrent generation. ${ }^{[45]}$ The current study suggests that nanocrystal QDs with metal chalcogenide ligands may be better suited for solar energy conversion in solar cells than use in light-emitting devices as luminophores;

4. QDs with inorganic ligands for energy conversions (Chapter 5)

* The effects of organic-to-inorganic ligand exchange on the electronic and optoelectronic properties of colloidal CdSe core and core/shell QDs were investigated;

* To evaluate of $\mathrm{SnS}_{4}$-capped QDs for light emission application, $\mathrm{SnS}_{4}$-capped $\mathrm{CdSe} / \mathrm{CdS} / \mathrm{Zn}_{0.5} \mathrm{Cd}_{0.5} \mathrm{~S} / \mathrm{ZnS}$ core/multishell QDs were assembled into films using 
LBL assembly with PDDA as the linker. The QD-LEDs were fabricated by sandwiching $\mathrm{SnS}_{4}$-capped QDs/PDDA films between ITO and aluminum electrodes. The LEDs had a low turn-on voltage of $\sim 4 \mathrm{~V}$ and stable EL matching well with the PL spectrum of the QD film. However, due to the strong conductivity and poor passivation properties of $\mathrm{SnS}_{4}$ ligands, the efficiency of QD-LEDs is significantly lower than similar LEDs based on CdSe QDs with original organic ligands;

* A simple ITO/SnS 4 -capped CdSe core QDs/Al structure was used to evaluate of $\mathrm{SnS}_{4}$-capped QDs for photovoltaic conversion. The devices showed much higher electrical conductivity and reduced space-charge limited current after the ligand exchange, indicating that the small inorganic $\mathrm{SnS}_{4}$ ligands resulted in enhanced inter-QD electronic coupling. Meanwhile, the $\mathrm{SnS}_{4}$-capped QDs were found to retain strong excitonic absorption. Under illumination, the photocurrent response of an ITO/QDs/Al structure increased by several orders of magnitude after the ligand exchange. These findings bode well for the applicability of colloidal QDs with metal chalcogenide ligands as functional blocks to achieve efficient photodetection and solar energy conversion at low processing temperatures;

\subsection{Future Work}

Since direct injection of carriers into QDs is very difficult due to their low valance band energies, exciton energy transfer plays an important role in the EL of QD-LEDs. Further work to enhance the performance of QD-LEDs should focus on developing new strategies for efficient energy transfer between QDs and organic molecules. In our previous work, the EL of CdSe/ZnS QD-LEDs was enhanced by a factor of 2.5 with phosphorescent donors, but the EQE of the QDLEDs was still relatively low even with an optimized FIrpic donor layer. Two ways are proposed here to further improve the performance of CdSe/ZnS QD-LEDs with phosphorescent donors: 
(1) In the CBP-FIrpic host-guest system, triplet energy transfer is an endothermic process, limiting the exciton harvesting rate. Replacing CBP with a host having a higher triplet energy, such as $\mathrm{N}$, N'- dicarbazolyl-3,5-benzene (mCP), will enable exothermic energy transfer to FIrpic, ${ }^{[79]}$ and thus enhance the subsequent energy transfer to QDs

(2) The QD layer and QD-LED structure used in this study are not optimized to realize the maximal exciton generation and energy transfer. The QD layer acts as a hole blocking layer, limiting the number of injected holes and thus the number of excitons formed in the CBP layer. An ideal hybrid structure would consist of a random mixture of QDs and phosphorescent donors with an appropriate mole ratio, embedded in a host layer.

Another important finding of our study is that the photocurrent in an ITO/CdSe QDs/Al device with $\mathrm{SnS}_{4}$-capped QDs was more than two orders of magnitude higher than that in a similar device with organic ligands, mainly due to facile charge separation and transport in the all-inorganic QD solids. However, the peak EQE of this device was only $\sim 0.2 \%$. The poor yield of photocurrent generation may be attributed to the fact that there is a lack of driving forces which can efficiently separate photogenerated excitons and drive electrons toward the $\mathrm{Al}$ electrode in this simple device structure, as evidenced by a low open-circuit voltage (tens of $\mathrm{mV}$ ). To realize more efficient solar energy conversion based on QDs with inorganic ligands, new device architectures which facilitate exciton dissociation must be developed. For example, CdSe QDs may be combined with CdTe nanocrystals or a ZnO layer to form a type-II heterojunction, where photogenerated excitons can dissociate efficiently through donor-acceptor charge transfer. ${ }^{[96]}$ Such solar cell structures would benefit more from the facile charge transport through the inorganically-capped QD solids, and exhibit a higher energy-conversion efficiency. 


\section{Appendix}

\section{A.1 Synthesis of CdSe/ZnS Core/Shell Quantum Dots with 5 Monolayers of ZnS Shell}

\section{A.1.1 Synthesis of CdSe Core QDs}

A modified hot-injection method was used to synthesize CdSe core QDs. Typically, the mixture of $1 \mathrm{mmol}$ (128.4 mg) CdO, $1.27 \mathrm{~mL} \mathrm{OA}$ and $10 \mathrm{~mL}$ ODE in a three-necked flask was evacuated for $30 \mathrm{~min}$ (20 $\mathrm{min}$ at room temperature and $10 \mathrm{~min}$ at $100{ }^{\circ} \mathrm{C}$ ) and heated to about $250{ }^{\circ} \mathrm{C}$ under $\mathrm{N}_{2}$ atmosphere to obtain a clear solution. After the solution was cooled to room temperature, ODA (7.5 g) and TOPO (2.5 g) were added and the solution was reheated to $280{ }^{\circ} \mathrm{C}$. At this temperature, a selenium solution made by dissolving $8 \mathrm{mmol}$ (631.68 $\mathrm{mg}$ ) selenium in TOP (8 mL) and ODE (2 mL) was quickly injected. After the injection, the temperature was adjusted to $260{ }^{\circ} \mathrm{C}$ for 40 sec to allow the growth of CdSe QDs. After this time, the heating mantle was removed to cool the solution to room temperature, and an extraction procedure was used to purify the QDs from side products and unreacted precursors ${ }^{[32]}$. After this extraction procedure, the QDs were dispersed in hexane for further processing. The procedure typically generates CdSe core QDs with the first excitonic absorption peak around $530 \mathrm{~nm}$, that is, with a diameter of about $2.7 \mathrm{~nm}$. The PL QY of CdSe core QDs is around 9.5\%.

\section{A.1.2 Preparation of the Precursor Solution for the SILAR Method}

For shell growth using the SILAR method, ${ }^{[28]}$ we used precursor solutions containing only metal oxides $(\mathrm{ZnO})$, and elemental sulfur. The zinc precursor solution (0.04 M) was 
prepared by dissolving $\mathrm{ZnO}(81.39 \mathrm{mg})$ in $\mathrm{OA}(2.54 \mathrm{~mL})$ and $\mathrm{ODE}(22.46 \mathrm{~mL})$ at $310^{\circ} \mathrm{C}$. The sulfur precursor solution $(0.04 \mathrm{M})$ was prepared by dissolving sulfur in ODE at $180{ }^{\circ} \mathrm{C}$. All of the solutions were freshly prepared under $\mathrm{N}_{2}$ atmosphere. The Zn-precursor solutions were kept at about $80^{\circ} \mathrm{C}$, while the sulfur injection solution was allowed to cool to room temperature. For each shell growth, a calculated amount of a given precursor solution was injected with a syringe using standard air-free procedures.

\section{A.1.3 Calculations of the Amount of Precursor Solutions for Shell \\ Growth}

The growth of CdSe/ZnS core/shell QDs using the SILAR method is based on alternating injections of the Zn- and S-precursors into the solution containing the CdSe core QDs. The concentration of CdSe core QDs can be estimated on the basis of the absorption spectra. ${ }^{[32]}$ The amount of the injection solution for each monolayer can be deduced from a calculation of the number of surface atoms:

The size of CdSe core: $\quad \mathrm{D}_{0}=2.68 \mathrm{~nm} \quad \mathrm{R}_{0}=1.34 \mathrm{~nm}$

The molar quantity of CdSe core QDs: $\mathrm{n}_{1}=7.35 \times 10^{-7} \mathrm{~mol}$

The average thickness of one monolayer of $\mathrm{ZnS}(\mathbf{d})$ : $\quad d=0.31 \mathrm{~nm}$

The amount of $\mathrm{Zn}$ and S precursor of the first layer $\mathrm{ZnS}$ (1ML):

The volume of the first ZnS monolayer: $\mathrm{V}_{1}=4 \pi\left(\mathrm{R}_{1}{ }^{3}-\mathrm{R}_{0}{ }^{3}\right) / 3$

$$
\begin{aligned}
& \mathrm{R}_{0}=1.34 \mathrm{~nm} \quad \mathrm{R}_{1}=1.34+0.31=1.65 \mathrm{~nm} \\
& \mathrm{~V}_{1}=4 \times 3.14 \times\left(1.65^{3}-1.34^{3}\right) / 3=8.7498 \mathrm{~nm}^{3}=8.7498 \times 10^{-27} \mathrm{~m}^{3}
\end{aligned}
$$

The density of ZnS material: $\quad \mathrm{D}=4110 \mathrm{lg} / \mathrm{m}^{3}$

The mass of the first ML ZnS shell for a single core/shell QD:

$$
\begin{gathered}
\mathrm{m}=\text { Volume } \times \text { Density } \\
\mathrm{m}=8.7498 \times 10^{-27} \mathrm{~m}^{3} \times 4110 \mathrm{~kg} / \mathrm{m}^{3}=3.596182 \times 10^{-23} \mathrm{~kg}
\end{gathered}
$$


The molar quantity of ZnS for a single core/shell QD: $n=m / M . W$.

$$
\begin{gathered}
\text { M.W. }(\text { Molecular Weight })=9.746 \times 10^{-2} \mathrm{~kg} / \mathrm{mol} \\
\mathrm{n}_{2}=\mathrm{m} / \mathrm{M} . \mathrm{W} .=3.596182 \times 10^{-23} \mathrm{~kg} / 9.746 \times 10^{-2} \mathrm{~kg} / \mathrm{mol}=3.6899 \times 10^{-22} \mathrm{~mol} / \mathrm{QD}
\end{gathered}
$$

The molar quantity of Zn-/S- precursor for the first ML ZnS growth:

$$
\begin{gathered}
\mathrm{A}_{1}=\mathrm{n}_{1} \times \mathrm{N} \times \mathrm{n}_{2} \\
\mathrm{~A}_{1}=7.35 \times 10^{-7} \mathrm{~mol} \times\left(6.02 \times 10^{23} \mathrm{QD} / \mathrm{mol}\right) \times 3.6899 \times 10^{-22} \mathrm{~mol} / \mathrm{QD}=1.63267 \times 10^{-4} \mathrm{~mol} \\
=0.163267 \mathrm{mmol} \approx \mathbf{0 . 1 6 3} \mathbf{~ m m o l}
\end{gathered}
$$

2ML ZnS: $\mathrm{A}_{2}=\mathrm{A}_{1} \times \mathrm{V}_{2} / \mathrm{V}_{1}=0.163 \times\left(1.96^{3}-1.65^{3}\right) /\left(1.65^{3}-1.34^{3}\right)=\mathbf{0 . 2 3 8} \mathbf{~ m m o l}$

3ML ZnS: $\mathrm{A}_{3}=\mathrm{A}_{1} \times \mathrm{V}_{3} / \mathrm{V}_{1}=0.163 \times\left(2.27^{3}-1.96^{3}\right) /\left(1.65^{3}-1.34^{3}\right)=\mathbf{0 . 3 2 6} \mathbf{~ m m o l}$

4ML ZnS: $\mathrm{A}_{4}=\mathrm{A}_{1} \times \mathrm{V}_{4} / \mathrm{V}_{1}=0.163 \times\left(2.58^{3}-2.27^{3}\right) /\left(1.65^{3}-1.34^{3}\right)=\mathbf{0 . 4 2 8} \mathbf{~ m m o l}$

5ML ZnS: $\mathrm{A}_{5}=\mathrm{A}_{1} \times \mathrm{V}_{5} / \mathrm{V}_{1}=0.163 \times\left(2.89^{3}-2.58^{3}\right) /\left(1.65^{3}-1.34^{3}\right)=\mathbf{0 . 5 4 5} \mathbf{~ m m o l}$

For the actual CdSe/ZnS core/shell QDs growth, we assumed that the surface of the CdSe core consists equally of Se- and Cd-atoms, and therefore used only $50 \%$ of the molar quantity of calculated Zn-/S- precursor for a complete monolayer. ${ }^{[43]}$

\section{A.1.4 Synthesis of CdSe/ZnS Core/Shell QDs Using the SILAR Method}

A typical SILAR synthesis was performed as follows: $18 \mathrm{~mL}$ of ODE and $6 \mathrm{~g}$ of ODA were loaded into in a three-necked flask, heated to $100{ }^{\circ} \mathrm{C}$ under vacuum for $30 \mathrm{~min}$, and cooled to room temperature. The CdSe core QDs in hexane (2.68 nm in diameter, $\left.7.35 \times 10^{-7} \mathrm{~mol}\right)$ were added, and the system was kept at $100{ }^{\circ} \mathrm{C}$ under vacuum for $30 \mathrm{~min}$ to remove the hexane and other undesired materials of low vapor pressure. Subsequently, the solution was heated to $235^{\circ} \mathrm{C}$ under $\mathrm{N}_{2}$ atmosphere where the shell growth was performed. As calculated in section A.1.3, for the "first injection", we used only $2 \mathrm{~mL}$ instead of $4 \mathrm{~mL}$ of the Zn-precursor solution. As schematically shown in

Figure 2.11, this is followed by alternating addition of S-precursors and Zn-precursors, 
respectively, the amount of which was calculated from the respective volumes of concentric spherical shells with a thickness of one hypothetical monolayer. A period of 10 min between each addition was sufficient for the reaction to be completed. The complete coverage procedure of CdSe cores with 5 MLs of ZnS took about $2 \mathrm{~h}$ in total. After this time, the solution was cooled to room temperature. For purification, hexane was added and the unreacted compounds and byproducts were removed by successive methanol extraction ${ }^{[32]}$ (at least two times) until the methanol phase was clear.

\section{A.2 Characterization of Quantum Yields of Colloidal Quantum Dots}

The photoluminescence (PL) quantum yield (QY) is defined as follows:

$\mathrm{QY}=$ [number of PL photons emitted]/[number of incident photons absorbed]

Absolute measurements of QY are difficult, but it is straightforward to find the ratio of the QY for two substances, denoted as 1 and 2:

$$
\mathrm{QY}_{2} / \mathrm{QY}_{1}=\left[\mathrm{F}_{2} / \mathrm{A}_{2}\right] /\left[\mathrm{F}_{1} / \mathrm{A}_{1}\right]=\left[\mathrm{F}_{2} / \mathrm{F}_{1}\right] \times\left[\mathrm{A}_{1} / \mathrm{A}_{2}\right]
$$

Where $F_{1}=$ Integrated intensity of PL spectrum of the substance 1;

$\mathrm{F}_{2}=$ Integrated intensity of PL spectrum of the substance 2;

$\mathrm{A}_{1}=$ Absorbance at wavelength $\lambda_{\mathrm{a}}$ of absorption spectrum of substance 1;

$A_{2}=$ Absorbance at wavelength $\lambda_{\mathrm{a}}$ of absorption spectrum of substance 2;

The fluorescence spectrophotometer excites PL of the sample with incident light of a known wavelength $\lambda_{\mathrm{a}}$. The total number of PL photons emitted is proportional to the area under the PL intensity vs. wavelength of the PL spectrum. This integral area is the quantity F. The total number of incident photons that are absorbed during the PL process is proportional to the absorbance A at wavelength $\lambda_{\mathrm{a}}$, which can be measured by the UV-Vis absorption spectrometer. For best results in determining QYs, the two substances should have absorption peaks at approximately the same wavelengths, and their PL spectra should overlap as much as possible.

Therefore, PL QYs of the QD samples in hexane were calculated by comparing their 
integrated emission to that of a solution of organic dyes in methanol. The organic dye has a known QY. The PL QY data reported in this work were obtained using Rhodamine B as the standard for yellow, orange and red emission and R590 as the standard for green emitter. Absorbance of all solutions was adjusted to $\sim 0.08$ or slightly less at the excitation wavelength to avoid reabsorption effects. The excitation wavelength used for all measurements was $480 \mathrm{~nm}$. PL spectra were collected ranging from $490 \mathrm{~nm}$ to $700 \mathrm{~nm}$. 


\section{References}

[1] X. Peng, J. Wickham, A. Alivisatos, Kinetics of II-VI and III-V colloidal semiconductor nanocrystal growth: "focusing" of size distributions, Journal of the American Chemical Society 1998, 120, 5343-5344.

[2] C. Murray, D. Norris, M. G. Bawendi, Synthesis and characterization of nearly monodisperse CdE ( $E=$ sulfur, selenium, tellurium) semiconductor nanocrystallites, Journal of the American Chemical Society 1993, 115, 8706-8715.

[3] K. A. Abel, J. Shan, J. C. Boyer, F. Harris, F. C. J. M. van Veggel, Highly Photoluminescent PbS Nanocrystals: The Beneficial Effect of Trioctylphosphine, Chemistry of Materials 2008, 20, 3794-3796.

[4] D. V. Talapin, C. B. Murray, PbSe nanocrystal solids for n-and p-channel thin film fieldeffect transistors, Science 2005, 310, 86.

[5] J. Jeffrey, D. V. Talapin, E. V. Shevchenko, C. B. Murray, Self-assembly of PbTe quantum dots into nanocrystal superlattices and glassy films, Journal of the American Chemical Society 2006, 128, 3248-3255.

[6] D. Battaglia, X. Peng, Formation of high quality InP and InAs nanocrystals in a noncoordinating solvent, Nano Letters 2002, 2, 1027-1030.

[7] J. Shiang, A. Kadavanich, R. Grubbs, A. Alivisatos, Symmetry of annealed wurtzite CdSe nanocrystals: assignment to the C3v point group, The Journal of Physical Chemistry 1995, 99, 17417-17422.

[8] L. Esaki, R. Tsu, Superlattice and negative differential conductivity in semiconductors, IBM Journal of Research and Development 1970, 14, 61-65.

[9] V. Klimov, Nanocrystal Quantum dots: from fundamental phtophysics to multicolor lasing, Los Alamos Science 2003, 28, 214-220.

[10] M. Bruchez, M. Moronne, P. Gin, S. Weiss, A. P. Alivisatos, Semiconductor nanocrystals as fluorescent biological labels, Science 1998, 281, 2013.

[11] A. L. Rogach, N. Gaponik, J. M. Lupton, C. Bertoni, D. E. Gallardo, S. Dunn, N. Li Pira, M. Paderi, P. Repetto, S. G. Romanov, Light Emitting Diodes with Semiconductor Nanocrystals, Angewandte Chemie International Edition 2008, 47, 6538-6549.

[12] I. Gur, N. A. Fromer, M. L. Geier, A. P. Alivisatos, Air-stable all-inorganic nanocrystal solar cells processed from solution, Science 2005, 310, 462.

[13] D. C. Oertel, M. G. Bawendi, A. C. Arango, V. Bulović, Photodetectors based on treated CdSe quantum-dot films, Applied Physics Letters 2005, 87, 213505.

[14] A. M. Smith, S. Nie, Semiconductor nanocrystals: structure, properties, and band gap 
engineering, Accounts of Chemical Research 2009, 43, 190-200.

[15] D. V. Talapin, J. S. Lee, M. V. Kovalenko, E. V. Shevchenko, Prospects of colloidal nanocrystals for electronic and optoelectronic applications, Chemical Reviews 2010, 110, 389-458.

[16] D. V. Talapin, J. S. Lee, M. V. Kovalenko, E. V. Shevchenko, Prospects of colloidal nanocrystals for electronic and optoelectronic applications, Chemical Reviews 2009, 110, 389-458.

[17] L. Brus, A simple model for the ionization potential, electron affinity, and aqueous redox potentials of small semiconductor crystallites, The Journal of Chemical Physics 1983, 79, 5566.

[18] M. L. Steigerwald, L. E. Brus, Semiconductor crystallites: a class of large molecules, Accounts of Chemical Research 1990, 23, 183-188.

[19] V. K. LaMer, R. H. Dinegar, Theory, production and mechanism of formation of monodispersed hydrosols, Journal of the American Chemical Society 1950, 72, 48474854.

[20] N. Gaponik, S. G. Hickey, D. Dorfs, A. L. Rogach, A. Eychmüller, Progress in the light emission of colloidal semiconductor nanocrystals, Small 2010, 6, 1364-1378.

[21] Z. A. Peng, X. Peng, Formation of high-quality CdTe, CdSe, and CdS nanocrystals using CdO as precursor, Journal of the American Chemical Society 2001, 123, 183-184.

[22] L. Qu, Z. A. Peng, X. Peng, Alternative routes toward high quality CdSe nanocrystals, Nano Letters 2001, 1, 333-337.

[23] X. Peng, M. C. Schlamp, A. V. Kadavanich, A. Alivisatos, Epitaxial growth of highly luminescent CdSe/CdS core/shell nanocrystals with photostability and electronic accessibility, Journal of the American Chemical Society 1997, 119, 7019-7029.

[24] P. Reiss, J. Bleuse, A. Pron, Highly luminescent CdSe/ZnSe core/shell nanocrystals of low size dispersion, Nano Letters 2002, 2, 781-784.

[25] M. Schlamp, X. Peng, A. Alivisatos, Improved efficiencies in light emitting diodes made with CdSe (CdS) core/shell type nanocrystals and a semiconducting polymer, Journal of applied physics 1997, 82, 5837.

[26] P. Reiss, M. Protière, L. Li, Core/shell semiconductor nanocrystals, Small 2009, 5, 154168.

[27] M. A. Hines, P. Guyot-Sionnest, Synthesis and characterization of strongly luminescing ZnS-capped CdSe nanocrystals, The Journal of Physical Chemistry 1996, 100, 468-471.

[28] J. J. Li, Y. A. Wang, W. Guo, J. C. Keay, T. D. Mishima, M. B. Johnson, X. Peng, Largescale synthesis of nearly monodisperse CdSe/CdS core/shell nanocrystals using air-stable reagents via successive ion layer adsorption and reaction, Journal of the American Chemical Society 2003, 125, 12567-12575.

[29] Q. Sun, Y. A. Wang, L. S. Li, D. Wang, T. Zhu, J. Xu, C. Yang, Y. Li, Bright, multicoloured light-emitting diodes based on quantum dots, Nature Photonics 2007, 1, 
717-722.

[30] S. Xu, H. Shen, C. Zhou, Y. Hang, C. Liu, H. Wang, L. Ma, L. S. Li, Effect of Shell Thickness on the Optical Properties in CdSe/CdS/Zn0. 5Cd0. 5S/ZnS and CdSe/CdS/ZnxCd1-xS/ZnS Core/Multishell Nanocrystals, The Journal of Physical Chemistry C 2011.

[31] J. Zhao, J. A. Bardecker, A. M. Munro, M. S. Liu, Y. Niu, I. K. Ding, J. Luo, B. Chen, A. K. Y. Jen, D. S. Ginger, Efficient CdSe/CdS quantum dot light-emitting diodes using a thermally polymerized hole transport layer, Nano Letters 2006, 6, 463-467.

[32] W. W. Yu, L. Qu, W. Guo, X. Peng, Experimental determination of the extinction coefficient of CdTe, CdSe, and CdS nanocrystals, Chemistry of Materials 2003, 15, 28542860.

[33] Y. H. Niu, A. M. Munro, Y. J. Cheng, Y. Tian, M. S. Liu, J. Zhao, J. A. Bardecker, I. Jen La Plante, D. S. Ginger, A. K. Y. Jen, Improved Performance from Multilayer Quantum Dot Light Emitting Diodes via Thermal Annealing of the Quantum Dot Layer, Advanced Materials 2007, 19, 3371-3376.

[34] C. Murray, C. Kagan, M. Bawendi, Synthesis and characterization of monodisperse nanocrystals and close-packed nanocrystal assemblies, Annual Review of Materials Science 2000, 30, 545-610.

[35] E. A. Weiss, R. C. Chiechi, S. M. Geyer, V. J. Porter, D. C. Bell, M. G. Bawendi, G. M. Whitesides, Size-dependent charge collection in junctions containing single-size and multi-size arrays of colloidal CdSe quantum dots, Journal of the American Chemical Society 2008, 130, 74-82.

[36] D. Yu, C. Wang, P. Guyot-Sionnest, n-Type conducting CdSe nanocrystal solids, Science 2003, 300, 1277-1280.

[37] J. M. Luther, M. Law, M. C. Beard, Q. Song, M. O. Reese, R. J. Ellingson, A. J. Nozik, Schottky solar cells based on colloidal nanocrystal films, Nano Letters 2008, 8, 34883492.

[38] J. M. Luther, M. Law, Q. Song, C. L. Perkins, M. C. Beard, A. J. Nozik, Structural, optical, and electrical properties of self-assembled films of PbSe nanocrystals treated with 1, 2-ethanedithiol, ACS Nano 2008, 2, 271-280.

[39] C. Leatherdale, C. Kagan, N. Morgan, S. Empedocles, M. Kastner, M. Bawendi, Photoconductivity in CdSe quantum dot solids, Physical Review B 2000, 62, 2669.

[40] M. V. Kovalenko, D. V. Talapin, M. A. Loi, F. Cordella, G. Hesser, M. I. Bodnarchuk, W. Heiss, Quasi Seeded Growth of Ligand Tailored PbSe Nanocrystals through Cation Exchange Mediated Nucleation, Angewandte Chemie International Edition 2008, 47, 3029-3033.

[41] G. Konstantatos, J. Clifford, L. Levina, E. H. Sargent, Sensitive solution-processed visible-wavelength photodetectors, Nature Photonics 2007, 1, 531-534.

[42] M. Soreni-Harari, N. Yaacobi-Gross, D. Steiner, A. Aharoni, U. Banin, O. Millo, N. 
Tessler, Tuning energetic levels in nanocrystal quantum dots through surface manipulations, Nano Letters 2008, 8, 678-684.

[43] R. Xie, U. Kolb, J. Li, T. Basché, A. Mews, Synthesis and characterization of highly luminescent CdSe-Core CdS/Zn0. 5Cd0. 5S/ZnS multishell nanocrystals, Journal of the American Chemical Society 2005, 127, 7480-7488.

[44] K. S. Cho, E. K. Lee, W. J. Joo, E. Jang, T. H. Kim, S. J. Lee, S. J. Kwon, J. Y. Han, B. K. Kim, B. L. Choi, High-performance crosslinked colloidal quantum-dot light-emitting diodes, Nature Photonics 2009, 3, 341-345.

[45] E. Talgorn, E. Moysidou, R. D. Abellon, T. J. Savenije, A. Goossens, A. J. Houtepen, L. D. A. Siebbeles, Highly photoconductive CdSe quantum-dot films: influence of capping molecules and film preparation procedure, The Journal of Physical Chemistry C 2010, 114, 3441-3447.

[46] M. V. Kovalenko, M. Scheele, D. V. Talapin, Colloidal nanocrystals with molecular metal chalcogenide surface ligands, Science 2009, 324, 1417.

[47] M. V. Kovalenko, M. I. Bodnarchuk, J. Zaumseil, J. S. Lee, D. V. Talapin, Expanding the Chemical Versatility of Colloidal Nanocrystals Capped with Molecular Metal Chalcogenide Ligands, Journal of the American Chemical Society 2010, 132, 1008510092.

[48] A. Nag, M. V. Kovalenko, J. S. Lee, W. Liu, B. Spokoyny, D. V. Talapin, Metal-free Inorganic Ligands for Colloidal Nanocrystals: $\mathrm{S}^{2-}, \mathrm{HS}^{-}, \mathrm{Se}^{2-}, \mathrm{HSe}^{-}, \mathrm{Te}^{2-}, \mathrm{HTe}^{-}, \mathrm{TeS}_{3}{ }^{2-}$, $\mathrm{OH}$, and $\mathrm{NH}_{2}^{-}$as Surface Ligands, Journal of the American Chemical Society 2011, 133, 10612-10620.

[49] J. S. Lee, M. V. Kovalenko, J. Huang, D. S. Chung, D. V. Talapin, Band-like transport, high electron mobility and high photoconductivity in all-inorganic nanocrystal arrays, Nature Nanotechnology 2011, 6, 348-352.

[50] A. L. Rogach, A. Eychmüller, S. G. Hickey, S. V. Kershaw, Infrared - Emitting Colloidal Nanocrystals: Synthesis, Assembly, Spectroscopy, and Applications, Small 2007, 3, 536557.

[51] S. Coe-Sullivan, Optoelectronics: Quantum dot developments, Nature Photonics 2009, 3, 315-316.

[52] V. Colvin, M. Schlamp, A. Alivisatos, Light-emitting diodes made from cadmium selenide nanocrystals and a semiconducting polymer, Nature 1994, 370, 354-357.

[53] W. K. W. Seth Coe, V. B. Moungi Bawendi, Electroluminescence from single monolayers of nanocrystals in molecular organic devices, Nature 2002, 420, 800-803.

[54] S. Coe Sullivan, J. S. Steckel, W. K. Woo, M. G. Bawendi, V. Bulovi Large Area Ordered Quantum Dot Monolayers via Phase Separation During Spin Casting, Advanced Functional Materials 2005, 15, 1117-1124.

[55] P. O. Anikeeva, J. E. Halpert, M. G. Bawendi, V. Bulovic, Quantum dot light-emitting devices with electroluminescence tunable over the entire visible spectrum, Nano Letters 
2009, 9, 2532-2536.

[56] T. H. Kim, K. S. Cho, E. K. Lee, S. J. Lee, J. Chae, J. W. Kim, D. H. Kim, J. Y. Kwon, G. Amaratunga, S. Y. Lee, Full-colour quantum dot displays fabricated by transfer printing, Nature Photonics 2011.

[57] Y. Wu, C. Wadia, W. Ma, B. Sadtler, A. P. Alivisatos, Synthesis and photovoltaic application of copper (I) sulfide nanocrystals, Nano Letters 2008, 8, 2551-2555.

[58] J. Tang, S. Hinds, S. O. Kelley, E. H. Sargent, Synthesis of colloidal CuGaSe2, CuInSe2, and Cu (InGa) Se2 nanoparticles, Chemistry of Materials 2008, 20, 6906-6910.

[59] Q. Guo, S. J. Kim, M. Kar, W. N. Shafarman, R. W. Birkmire, E. A. Stach, R. Agrawal, H. W. Hillhouse, Development of CuInSe2 nanocrystal and nanoring inks for low-cost solar cells, Nano Letters 2008, 8, 2982-2987.

[60] K. W. Johnston, A. G. Pattantyus-Abraham, J. P. Clifford, S. H. Myrskog, S. Hoogland, H. Shukla, E. J. D. Klem, L. Levina, E. H. Sargent, Efficient Schottky-quantum-dot photovoltaics: The roles of depletion, drift, and diffusion, Applied Physics Letters 2008, 92, 122111.

[61] G. I. Koleilat, L. Levina, H. Shukla, S. H. Myrskog, S. Hinds, A. G. Pattantyus-Abraham, E. H. Sargent, Efficient, stable infrared photovoltaics based on solution-cast colloidal quantum dots, ACS nano 2008, 2, 833-840.

[62] Z. Li, H. Meng, Organic light-emitting materials and devices, Taylor \& Francis, 2007.

[63] S. S. Sun, N. S. Sariciftci, Organic Photovoltaics Mechanisms, Materials, and Devices, Taylor \& Francis, 2005.

[64] J. Jasieniak, M. Califano, S. Watkins, Size-Dependent Valence and Conduction BandEdge Energies of Semiconductor Nanocrystals, ACS nano 2011, 5, 5888-5902.

[65] B. Dabbousi, J. Rodriguez-Viejo, F. Mikulec, J. Heine, H. Mattoussi, R. Ober, K. Jensen, M. Bawendi, (CdSe) ZnS core-shell quantum dots: synthesis and characterization of a size series of highly luminescent nanocrystallites, The Journal of Physical Chemistry B 1997, 101, 9463-9475.

[66] D. V. Talapin, I. Mekis, S. Goetzinger, A. Kornowski, O. Benson, H. Weller, CdSe/CdS/ZnS and CdSe/ZnSe/ZnS core-shell-shell nanocrystals, The Journal of Physical Chemistry B 2004, 108, 18826-18831.

[67] J. Carter, I. Grizzi, S. Heeks, D. Lacey, S. Latham, P. May, O. R. de los Panos, K. Pichler, C. Towns, H. Wittmann, Operating stability of light-emitting polymer diodes based on poly (p-phenylene vinylene), Applied Physics Letters 1997, 71, 34.

[68] Q. Sun, B. Fan, Z. Tan, C. Yang, Y. Li, Y. Yang, White light from polymer light-emitting diodes: Utilization of fluorenone defects and exciplex, Applied Physics Letters 2006, 88, 163510.

[69] Q. Sun, J. Hou, C. Yang, Y. Li, Y. Yang, Enhanced performance of white polymer lightemitting diodes using polymer blends as hole-transporting layers, Applied Physics Letters 2006, 89, 153501-153503. 
[70] C. Tang, S. VanSlyke, Organic electroluminescent diodes, Applied Physics Letters 1987, 51, 913-915.

[71] Y.Z hang, X. Cao, Electroluminescence of green CdSe/ZnS quantum dots enhanced by harvesting excitons from phosphorescent molecules, Applied Physics Letters 2010, 97, 253115-253115-253113.

[72] P. Anikeeva, C. Madigan, J. Halpert, M. Bawendi, V. Bulovi Electronic and excitonic processes in light-emitting devices based on organic materials and colloidal quantum dots, Physical Review B 2008, 78, 085434.

[73] P. Anikeeva, C. Madigan, S. Coe-Sullivan, J. Steckel, M. Bawendi, V. Bulovic, Photoluminescence of CdSe/ZnS core/shell quantum dots enhanced by energy transfer from a phosphorescent donor, Chemical physics letters 2006, 424, 120-125.

[74] A. R. Clapp, I. L. Medintz, B. R. Fisher, G. P. Anderson, H. Mattoussi, Can luminescent quantum dots be efficient energy acceptors with organic dye donors?, Journal of the American Chemical Society 2005, 127, 1242-1250.

[75] N. Matsusue, Y. Suzuki, H. Naito, Charge carrier transport in neat thin films of phosphorescent iridium complexes, Japanese journal of applied physics 2005, 44, 36913694.

[76] D. L. Dexter, A theory of sensitized luminescence in solids, The Journal of Chemical Physics 1953, 21, 836.

[77] T. Förster, Zwischenmolekulare energiewanderung und fluoreszenz, Annalen der Physik 1948, 437, 55-75.

[78] Y. Q. Zhang, G. Y. Zhong, X. A. Cao, Concentration quenching of Electroluminescence in Neat Ir(ppy)3 Organic Light Emitting Diodes, J. Appl. Phys. 2010, 108, 083107.

[79] R. Holmes, S. Forrest, Y. J. Tung, R. Kwong, J. Brown, S. Garon, M. Thompson, Blue organic electrophosphorescence using exothermic host-guest energy transfer, Applied Physics Letters 2003, 82, 2422.

[80] M. Kuno, J. Lee, B. Dabbousi, F. Mikulec, M. Bawendi, The band edge luminescence of surface modified CdSe nanocrystallites: Probing the luminescing state, The Journal of Chemical Physics 1997, 106, 9869.

[81] D. Yu, C. Wang, P. Guyot-Sionnest, n-type conducting CdSe nanocrystal solids, Science 2003, 300, 1277.

[82] N. Y. Morgan, C. Leatherdale, M. Drndi , M. V. Jarosz, M. A. Kastner, M. Bawendi, Electronic transport in films of colloidal CdSe nanocrystals, Physical Review B 2002, 66, 075339.

[83] G. Konstantatos, I. Howard, A. Fischer, S. Hoogland, J. Clifford, E. Klem, L. Levina, E. H. Sargent, Ultrasensitive solution-cast quantum dot photodetectors, Nature 2006, 442, 180-183.

[84] W. K. Bae, J. Kwak, J. Lim, D. Lee, M. K. Nam, K. Char, C. Lee, S. Lee, Multicolored Light-Emitting Diodes Based on All-Quantum-Dot Multilayer Films Using Layer-by- 
Layer Assembly Method, Nano Letters 2010, 10, 2368-2373.

[85] B. A. Ridley, B. Nivi, J. M. Jacobson, All-inorganic field effect transistors fabricated by printing, Science 1999, 286, 746.

[86] M. Drndi , M. Jarosz, N. Morgan, M. Kastner, M. Bawendi, Transport properties of annealed CdSe colloidal nanocrystal solids, Journal of applied physics 2002, 92, 7498.

[87] R. Chandler, A. Houtepen, J. Nelson, D. Vanmaekelbergh, Electron transport in quantum dot solids: Monte Carlo simulations of the effects of shell filling, Coulomb repulsions, and site disorder, Physical Review B 2007, 75, 085325.

[88] D. B. Mitzi, Solution processing of chalcogenide semiconductors via dimensional reduction, Advanced Materials 2009, 21, 3141-3158.

[89] E. H. Sargent, Colloidal quantum dot solar cells, Nature Photonics 2012, 6, 133-135.

[90] S. H. Kang, C. Kumar, Z. Lee, K. H. Kim, C. Huh, E. T. Kim, Quantum-dot light-emitting diodes utilizing CdSe/ZnS nanocrystals embedded in TiO2 thin film, Applied Physics Letters 2008, 93, 191116.

[91] N. Gaponik, A. L. Rogach, Thiol-capped CdTe nanocrystals: progress and perspectives of the related research fields, Phys. Chem. Chem. Phys. 2010, 12, 8685-8693.

[92] J. Tang, K. W. Kemp, S. Hoogland, K. S. Jeong, H. Liu, L. Levina, M. Furukawa, X. Wang, R. Debnath, D. Cha, Colloidal-quantum-dot photovoltaics using atomic-ligand passivation, Nature Materials 2011, 10, 765-771.

[93] S. Srivastava, N. A. Kotov, Composite layer-by-layer ( $L B L)$ assembly with inorganic nanoparticles and nanowires, Accounts of Chemical Research 2008, 41, 1831-1841.

[94] M. Gao, C. Lesser, S. Kirstein, H. Möhwald, A. L. Rogach, H. Weller, Electroluminescence of different colors from polycation/CdTe nanocrystal self-assembled films, Journal of Applied Physics 2000, 87, 2297-2302.

[95] D. Ginger, N. Greenham, Charge injection and transport in films of CdSe nanocrystals, Journal of Applied Physics 2000, 87, 1361.

[96] E. Talgorn, M. A. de Vries, L. D. A. Siebbeles, A. J. Houtepen, Photoconductivity Enhancement in Multilayers of CdSe and CdTe Quantum Dots, ACS Nano 2011, 5, 35523558.

[97] E. H. Sargent, Solar cells, photodetectors, and optical sources from infrared colloidal quantum dots, Advanced Materials 2008, 20, 3958-3964. 


\title{
Curriculum Vitae
}

\author{
YIQIANG ZHANG
}

zhangyiqiang808@gmail.com

\section{EDUCATION}

\author{
West Virginia University (WVU), U.S.A \\ Ph.D. in Electrical Engineering 2008-2012 \\ Dissertation title: “Colloidal Nanocrystal Quantum Dots for High-Efficiency Optoelectronic \\ Conversions"
}

Advisor: Xian-An Cao

Zhejiang University (ZJU), P R China

M. S. in Materials Science and Engineering 2005-2007

Thesis title: "Synthesis and Characterization of Monodispersed Metal Sulfide Nanocrystals" Advisor: Deren Yang and Duanlin Que

Zhengzhou University (ZZU), P. R. China

B.S. in Materials Science and Engineering 2001-2005

GPA: 3.68/4.0 Rank: $3^{\text {rd }} / 69$

\section{SCHOLARSHIPS \& HONORS}

1. Dissertation Fellowship, WVU, 2012

2. Chinese Government Award for Outstanding Self-Financed Students Abroad, 2011

3. CEMR College Student Travel Award, WVU, 2011

4. WVnano Bridge Award, WVU, 2009-2010

5. CSEE Department Fellowship, WVU, 2009

6. Excellent Graduate, ZZU, 2005

7. Honor of All-Round Student, ZZU, 2004

8. Outstanding Student Scholarship, ZZU, 2002-2004 


\section{JOURNAL PUBLICATIONS}

1. Y. Q. Zhang and X. A. Cao, "Evaluation of all-inorganic CdSe quantum dot thin films for optoelectronic applications", accepted by Nanotech.

2. Y. Q. Zhang, R. Acharya, and X. A. Cao, "Efficient and reliable green organic light-emitting diodes with $\mathrm{Cl}_{2}$ plasma-etched indium tin oxide anode", accepted by $\boldsymbol{J}$. Appl. Phys.

3. R. Acharya, Y. Q. Zhang, and X. A. Cao, "Characterization of zinc-tin-oxide films deposited by thermal co-evaporation", accepted by Thin Solid Films

4. X. A. Cao and Y. Q. Zhang, "Performance enhancement of organic light-emitting diodes by chlorine plasma treatment of indium tin oxide", Appl. Phys. Lett. 2012, 100, 183304

5. Y. Q. Zhang and X. A. Cao, "Optical characterization of CdSe quantum dots with metal chalcogenide ligands in solutions and solids", Appl. Phys. Lett. 2011, 99, 023106

6. X. A. Cao, Z. Y. Jiang and Y. Q. Zhang, "Organic thin film structures for high-sensitivity imaging of contact stress distributions", Org. Electron. 2011, 12, 306

7. Y. Q. Zhang and X. A. Cao, "Electroluminescence of green CdSe/ZnS quantum dots enhanced by harvesting excitons from phosphorescent molecules", Appl. Phys. Lett. 2010, 97, 253115

8. Y. Q. Zhang, G. Y. Zhong, and X. A. Cao, "Concentration quenching of electroluminescence in neat Ir(ppy)3 organic light-emitting diodes", J. Appl. Phys. 2010, 108, 083107

9. G. Y. Zhong, Y. Q. Zhang, and X. A. Cao, "Quenching-enhanced shift of recombination zone in phosphorescent organic light-emitting diodes", Org. Electron. 2010, 11, 1338

10. G. Y. Zhong, Y. Q. Zhang, and X. A. Cao, "Conjugated polymer films for piezoresistive stress sensing", Electron Device Letters, IEEE 2009, 30, 1137

11. Y. Q. Zhang, H. Zhang, J. X. Yu, D. R. Yang and D. L. Que, "Controllable Synthesis of Monodisperse CuS Nanocrystals” J. Mater. Sci. and Eng. 2008, 26, 208.

12. H. Zhang, Y. Q. Zhang, J. X. Yu and D. R. Yang, "Phase-Selective Synthesis and SelfAssembly of Monodisperse Copper Sulfide Nanodisks” J. Phys. Chem. C 2008, 112, 13390.

13. N. Du, H. Zhang, B. D. Chen, J.B. Wu, Z. H. Liu, Y. Q. Zhang, X. Y. Ma, D. R. Yang, X. H. Huang and J. P. Tu, "Porous $\mathrm{Co}_{3} \mathrm{O}_{4}$ Nanotubes Derived From $\mathrm{Co}_{4}(\mathrm{CO})_{12}$ Clusters on Carbon Nanotube Templates: A High Efficient Material For Li-Battery Application” Ad. Mater. 2007, 19, 4505.

\section{PROCEEDINGS PUBLICATIONS}

1. Y. Q. Zhang, R. Archaya, and X. A. Cao, "Evaluation of colloidal CdSe quantum dots with metal chalcogenide ligands for optoelectronic applications", Mater. Res. Soc. Symp. Proc., 2012, 426

2. Y. Q. Zhang and X. A. Cao, "Enhanced electroluminescence of CdSe/ZnS quantum dot light-emitting diodes with phosphorescent donors", Mater. Res. Soc. Symp. Proc., 2011, 1348 
3. X. A. Cao and Y. Q. Zhang, "Phosphorescent organic light-emitting devices to sense contact stresses", Mater. Res. Soc. Symp. Proc., 2011, 1358

\section{CONFERENCE PRESENTATIONS}

1. Y. Q. Zhang, R. Acharya, and X. A. Cao, "Evaluation of colloidal CdSe quantum dots with metal chalcogenide ligands for optoelectronic applications,” MRS Fall Meeting, Boston, Nov. 28-Dec 2, 2011 (poster)

2. Y. Q. Zhang and X. A. Cao, "Enhanced electroluminescence of CdSe/ZnS quantum dot light-emitting diodes with phosphorescent donors,” MRS Spring Meeting, San Francisco, Apr. 25-29, 2011 (oral presentation)

3. X. A. Cao and Y. Q. Zhang, "Phosphorescent organic light-emitting devices to sense contact stresses,” MRS Spring Meeting, San Francisco, Apr. 25-29, 2011 (oral presentation)

4. Y. Q. Zhang and X. A. Cao, "Development of efficient and stable Light-Emitting Diodes Based on Mircoconctact-Printing-Deposited CdSe Quantum Dots,” WVnano Res. Symp. Morgantown, WV, Apr. 20, 2010 (poster)

5. G. Y. Zhong, Y. Q. Zhang and X. A. Cao, "Investigation of self quenching effects in neat Ir(ppy)3 organic electrophosphorescent devices,” The 3rd Photonics and Opto-Electronics Meetings, Wuhan, China, Nov. 3-5, 2010 (oral presentation) 